Florida International University FIU Digital Commons

$11-4-2014$

\title{
Variable Speed Limit Strategies to Reduce the Impacts of Traffic Flow Breakdown at Recurrent Freeway Bottlenecks
}

Ali Darroudi

Florida International University, adarr001@fiu.edu

DOI: $10.25148 /$ etd.FI14110778

Follow this and additional works at: https:// digitalcommons.fiu.edu/etd

Part of the Civil Engineering Commons, Industrial Engineering Commons, and the Other Civil and Environmental Engineering Commons

\section{Recommended Citation}

Darroudi, Ali, "Variable Speed Limit Strategies to Reduce the Impacts of Traffic Flow Breakdown at Recurrent Freeway Bottlenecks" (2014). FIU Electronic Theses and Dissertations. 1705.

https://digitalcommons.fiu.edu/etd/1705

This work is brought to you for free and open access by the University Graduate School at FIU Digital Commons. It has been accepted for inclusion in FIU Electronic Theses and Dissertations by an authorized administrator of FIU Digital Commons. For more information, please contact dcc@fiu.edu. 


\section{FLORIDA INTERNATIONAL UNIVERSITY}

Miami, Florida

\section{VARIABLE SPEED LIMIT STRATEGIES TO REDUCE THE IMPACTS OF}

TRAFFIC FLOW BREAKDOWN AT RECURRENT FREEWAY BOTTLENECKS

A dissertation submitted in partial fulfillment of the

requirements for the degree of

DOCTOR OF PHILOSOPHY

in

CIVIL ENGINEERING

by

Ali Darroudi 


\section{To: Dean Amir Mirmiran}

College of Engineering and Computing

This dissertation, written by Ali Darroudi, and entitled Variable Speed Limit Strategies to Reduce the Impacts of Traffic Flow Breakdown at Recurrent Freeway Bottlenecks, having been approved in respect to style and intellectual content, is referred to you for judgment.

We have read this dissertation and recommend that it be approved.

L. David Shen

Albert Gan

Xia Jin

Shih-Ming Lee

Mohammed Hadi, Major Professor

Date of Defense: November 4, 2014

The dissertation of Ali Darroudi is approved.

$\begin{array}{r}\text { Dean Amir Mirmiran } \\ \text { College of Engineering and Computing } \\ \hline \begin{array}{r}\text { Dean Lakshmi N. Reddi } \\ \text { University Graduate School }\end{array}\end{array}$

Florida International University, 2014 


\section{DEDICATION}

To my parents, for their love and support throughout my life 


\section{ACKNOWLEDGMENTS}

My sincere gratitude and appreciation go first to my advisor, Dr. Mohammed Hadi, for his continued guidance, encouragement, inspiration, and support during my doctoral study. It was my honor to be mentored and led by such a well-respected researcher in the ITS field. What I have learned from Dr. Hadi will be invaluable to my future career and the rest of my life.

I am also grateful to the members of my dissertation committee, Dr. Albert Gan, Dr. David Shen, Dr. Xia Jin and Dr. Shih-Ming Lee for serving on my committee, and for their comments, suggestions, and interest in my research.

I experienced a great teamwork environment in the Integrated Intelligent Transportation Systems (IITS) research lab. I would like to extend my deep appreciation to Dr. Yan Xiao, who is incredibly wise, knowledgeable, and kind to share her knowledge.

I would like to acknowledge the Southeastern Transportation Research, Innovation, Development and Education Center (STRIDE) for funding this research. I am also grateful to have worked and collaborated with a research team from the University of Florida, including Dr. Lily Elefteriadou, Dr. Alexandra Kondyli, and Mr. Clark Letter. In addition, it should be mentioned that the base network and associated ramp metering used as the starting point in this research was coded by FDOT and later modified by the University of Florida’s researchers.

Finally, I would like to thank my parents for their encouragement and love. Without their patience, understanding, and support, the completion of this work would not have been possible. 


\title{
ABSTRACT OF THE DISSERTATION \\ VARIABLE SPEED LIMIT STRATEGIES TO REDUCE THE IMPACTS OF TRAFFIC FLOW BREAKDOWN AT RECURRENT FREEWAY BOTTLENECKS
}

by

\author{
Ali Darroudi \\ Florida International University, 2014 \\ Miami, Florida \\ Professor Mohammed Hadi, Major Professor
}

Variable Speed Limit (VSL) strategies identify and disseminate dynamic speed limits that are determined to be appropriate based on prevailing traffic conditions, road surface conditions, and weather conditions. This dissertation develops and evaluates a shockwave-based VSL system that uses a heuristic switching logic-based controller with specified thresholds of prevailing traffic flow conditions. The system aims to improve operations and mobility at critical bottlenecks. Before traffic breakdown occurrence, the proposed VSL's goal is to prevent or postpone breakdown by decreasing the inflow and achieving uniform distribution in speed and flow. After breakdown occurrence, the VSL system aims to dampen traffic congestion by reducing the inflow traffic to the congested area and increasing the bottleneck capacity by deactivating the VSL at the head of the congested area. The shockwave-based VSL system pushes the VSL location upstream as the congested area propagates upstream. In addition to testing the system using infrastructure detector-based data, this dissertation investigates the use of Connected Vehicle trajectory data as input to the shockwave-based VSL system performance. Since the field Connected Vehicle data are not available, as part of this research, 
Vehicle-to-Infrastructure communication is modeled in the microscopic simulation to obtain individual vehicle trajectories. In this system, wavelet transform is used to analyze aggregated individual vehicles' speed data to determine the locations of congestion.

The currently recommended calibration procedures of simulation models are generally based on the capacity, volume and system-performance values and do not specifically examine traffic breakdown characteristics. However, since the proposed VSL strategies are countermeasures to the impacts of breakdown conditions, considering breakdown characteristics in the calibration procedure is important to have a reliable assessment. Several enhancements were proposed in this study to account for the breakdown characteristics at bottleneck locations in the calibration process.

In this dissertation, performance of shockwave-based VSL is compared to VSL systems with different fixed VSL message sign locations utilizing the calibrated microscopic model. The results show that shockwave-based VSL outperforms fixed-location VSL systems, and it can considerably decrease the maximum back of queue and duration of breakdown while increasing the average speed during breakdown. 


\section{TABLE OF CONTENTS}

CHAPTER

PAGE

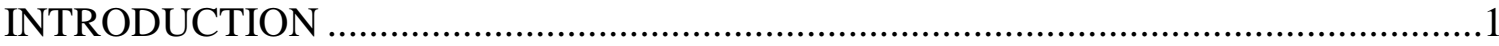

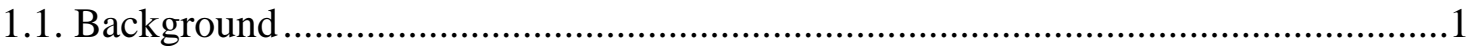

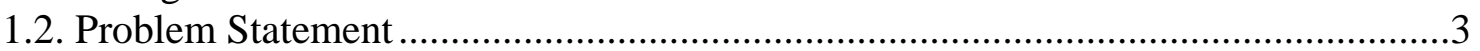

1.3. Research Goal and Objectives ...........................................................................

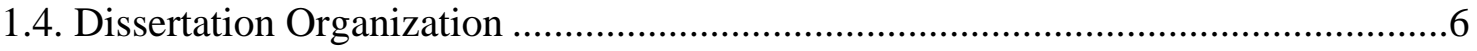

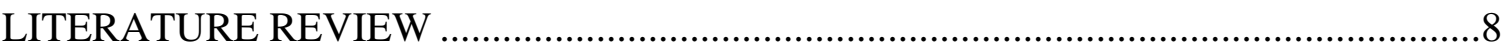

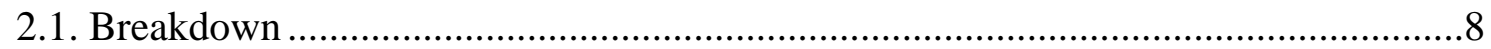

2.1.1. Breakdown Definition................................................................................

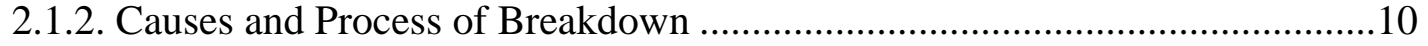

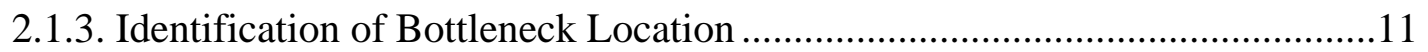

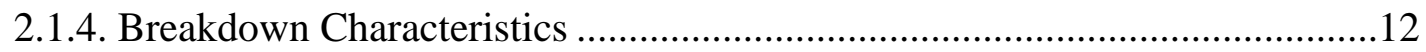

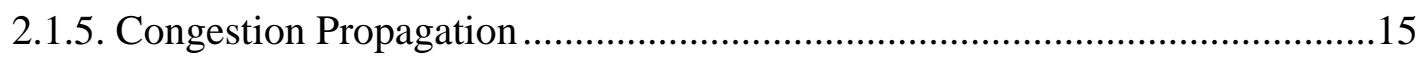

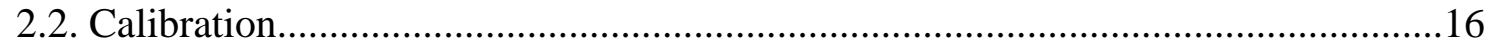

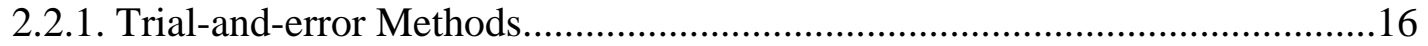

2.2.2. Heuristics-based Methods ..........................................................................20

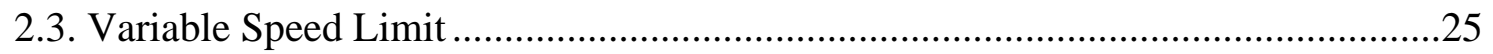

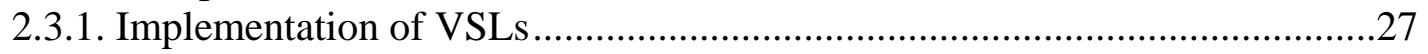

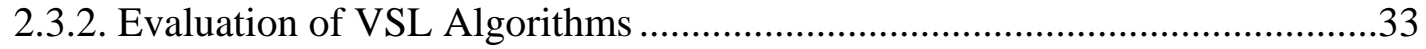

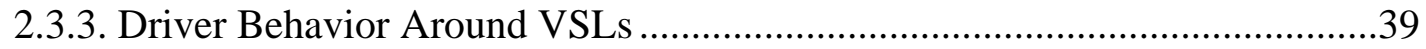

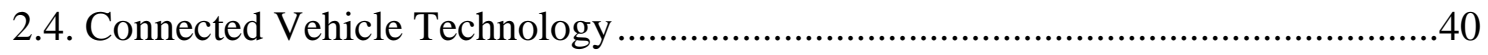

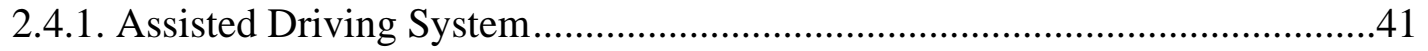

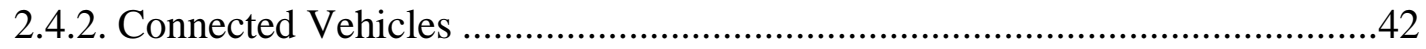

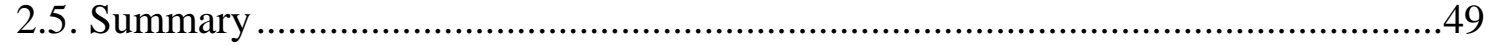

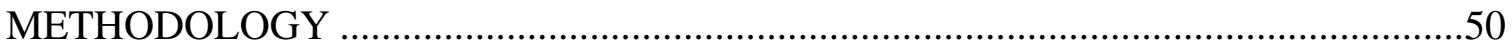

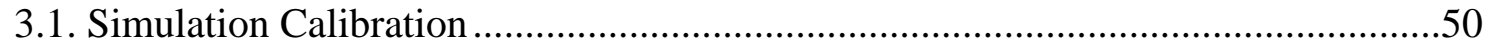

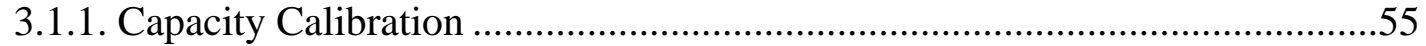

3.1.2. Traffic Volume Calibration.......................................................................58

3.1.3. Breakdown Characteristics Calibration ......................................................60

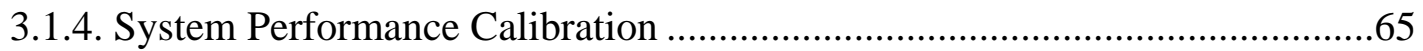

3.2. Background of the Developed VSL Methodology ..............................................67

3.3. VSL Strategy Based on Infrastructure Detector Data.........................................72

3.4. VSL Strategy Based on Connected Vehicle Data.................................................76

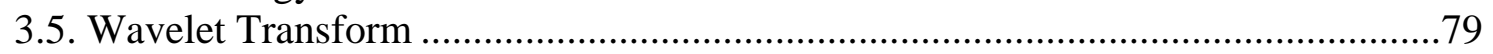

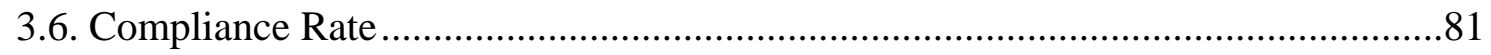

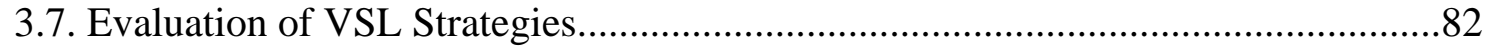

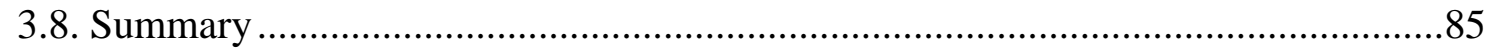

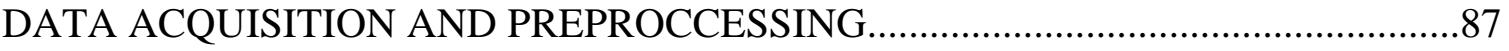

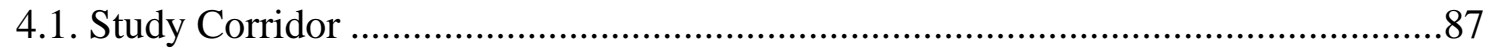

4.2. Infrastructure Detector Data Acquisition and Preprocessing................................87

4.3. Connected Vehicle Data Acquisition and Preprocessing.....................................96 


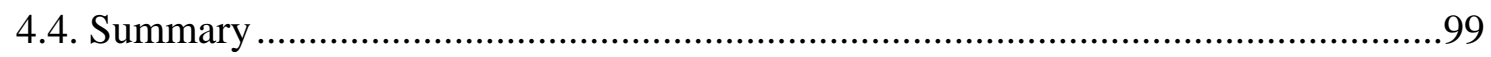

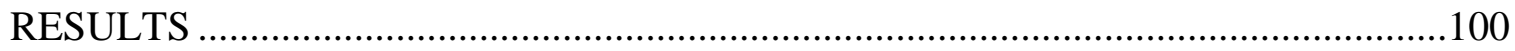

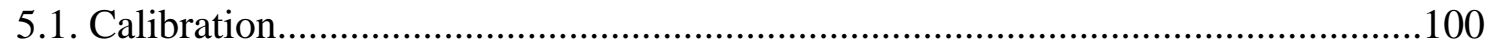

5.2. VSL Strategy Based on Infrastructure Detector Data.......................................111

5.3. VSL Strategy Based on Connected Vehicle Data............................................119

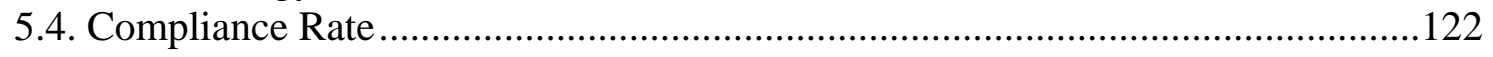

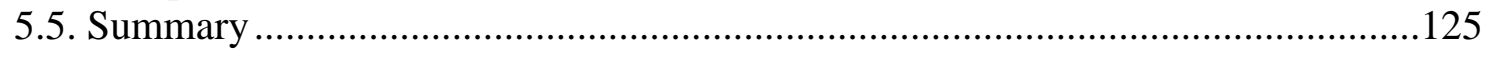

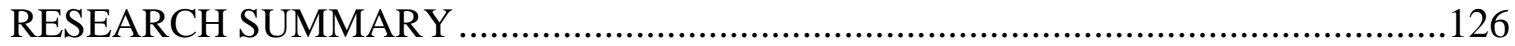

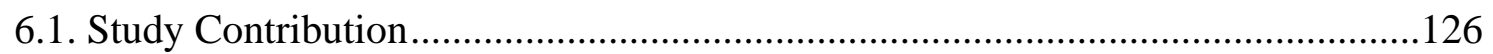

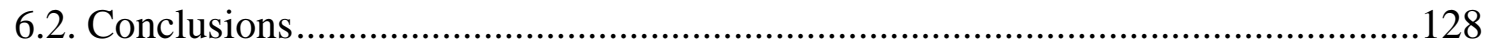

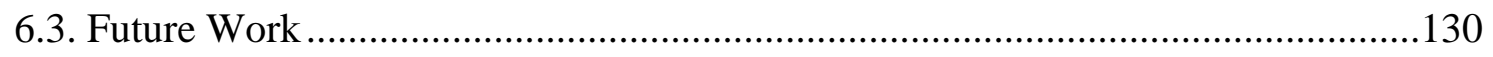

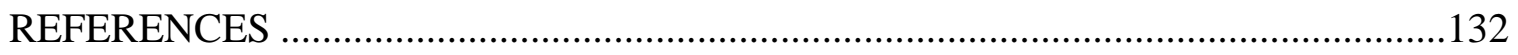

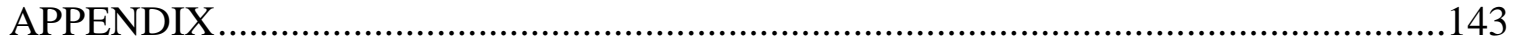

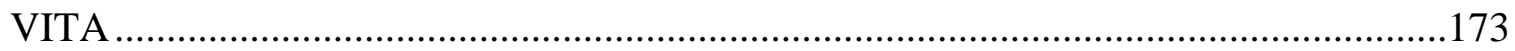




\section{LIST OF TABLES}

TABLE

PAGE

Table 4-1: Breakdown characteristics at first bottleneck based on real-world data 93

Table 4-2: Breakdown characteristics at second bottleneck based on real-world data..... 94

Table 4-3: Breakdown characteristics at third bottleneck based on real-world data ........ 95

Table 5-1: Average network speed for each simulation run ....................................... 102

Table 5-2: Breakdown characteristics at first bottleneck based on simulation result..... 103

Table 5-3: Breakdown characteristics at second bottleneck based on simulation result 103

Table 5-4: Breakdown characteristics at third bottleneck based on simulation result.... 104

Table 5-5: Breakdown characteristics comparison ................................................... 106

Table 5-6: Goodness-of-fit assessment of MOEs .................................................... 110

Table 5-7: Occupancy thresholds and sets of speed limits for traffic conditions ........... 114

Table 5-8: Congestion Index and maximum back of queue based different VSL

systems.

Table 5-9: Effects and improvements of each scenario on congestion......................... 117

Table 5-10: Breakdown conditions at the simulated bottleneck under different scenarios

Table 5-11: Congestion Index and maximum back of queue 121

Table 5-12: Breakdown conditions at bottleneck 121

Table 5-13: Breakdown conditions at bottleneck, different market penetration using VSL sign 124

Table 5-14: Breakdown conditions at bottleneck, different market penetration using Connected Vehicle.... 


\section{LIST OF FIGURES}

FIGURE

PAGE

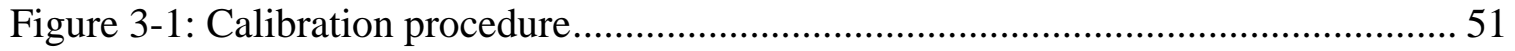

Figure 3-2: Capacity calibration procedure ........................................................... 55

Figure 3-3: Pre-breakdown flow and queue discharge rate estimation.......................... 57

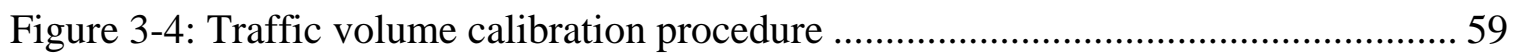

Figure 3-5: Breakdown characteristics calibration procedure ...................................... 61

Figure 3-6: Speed data of CORSIM output for one individual run and average of runs .. 64

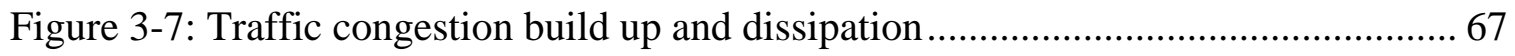

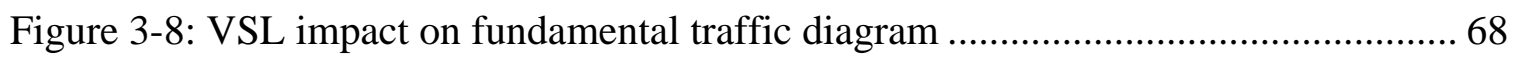

Figure 3-9: Speed-Flow curves for basic freeways segments under base conditions....... 71

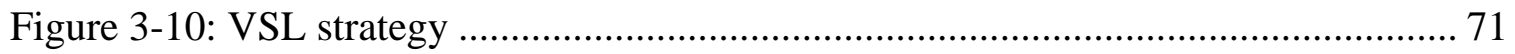

Figure 3-11: Traffic Regimes at Congested area ..................................................... 78

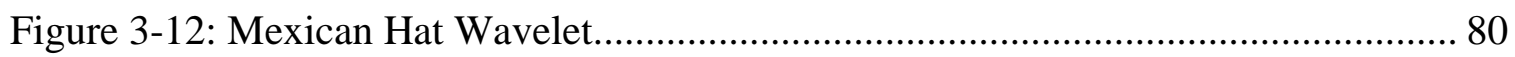

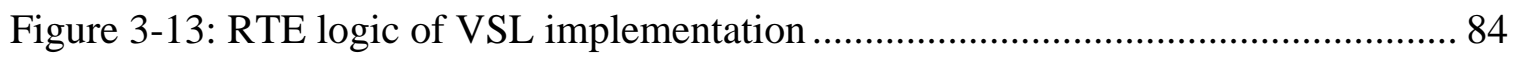

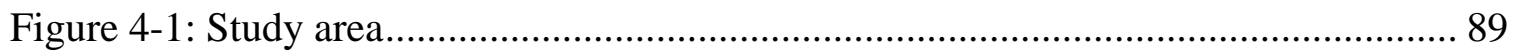

Figure 4-2: Speed contour maps based on real-world data........................................ 91

Figure 4-3: Illustration of wavelet transform and energy calculation............................ 92

Figure 4-4: Correlation coefficient of speed estimates based on collected data.............. 98

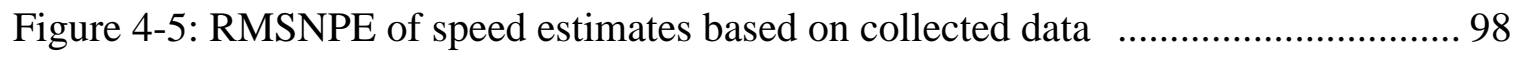

Figure 5-1: Screen shot of simulated network in CORSIM...................................... 101

Figure 5-2: Comparison of flow-occupancy relationship between real-world and

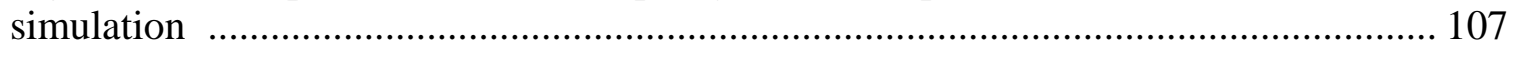

Figure 5-3: Comparison of breakdown probability between real-world and simulation 109

Figure 5-4: Speed contour map- simulation results ................................................ 110 
Figure 5-5: Third bottleneck scheme

Figure 5-6: Posted speed limits based on different strategies of data smoothing.....

Figure 5-7: Comparison of flow-occupancy relationship between before and after VSL

Figure 5-8: Comparison of congestion index based on different VSL scenarios ........... 116

Figure 5-9: Illustration of wavelet transform and energy calculation $\quad$........................... 120

Figure 5-10: Comparison of congestion index based on different compliance rate ....... 123 


\section{CHAPTER 1}

\section{INTRODUCTION}

\subsection{Background}

Road mobility significantly contributes to the society’s economy and welfare. Traffic congestion is a critical social issue that is faced every day; it cost drivers more than one hundred billion dollars in the United States in 2010 (Schrank et al., 2011). Recurrent congestion mainly occurs during peak periods when too many vehicles attempt to use a common roadway with limited capacity. Non-recurrent congestion also occurs due to incidents, special events, work zones, and weather events. Congestion is a source of productivity and efficiency loss, fuel wastage, and excessive air pollution. The areas that suffer most due to these problems are large urban areas, but even smaller urban and rural areas are starting to suffer from congestion.

Expanding road infrastructure is one of the solutions to traffic congestion. Because of the cost of construction, funding availability, and right-of-way and environmental concerns, many of the congested corridors will not have additional infrastructure built for many years to come. Meanwhile, it is important for transportation agencies and decision makers at the state, regional, and local levels to collectively invest in existing facilities and collaborate in the area of better managing their multimodal transportation corridors with improved operational strategies and technology.

To address these challenges and ensure a safe and efficient transportation system, transportation practitioners are looking for a more efficient use of existing road networks. Therefore, there is a tremendous need to understand the effects of different dynamic 
control methods on daily freeway operations and to identify and implement cost-effective control strategies. Active Traffic and Demand Management (ATDM) strategies such as variable speed limit (VSL) are state-of-the-art methods that are increasingly being considered to improve the efficiency of the existing freeway system. Connected Vehicle technologies have also been proposed to support more effective and efficient implementations.

The United States Department of Transportation (USDOT) Mobility Program has identified three high-priority transformative applications related to improving roadway operations through the utilization of frequently collected and rapidly disseminated multi-source data drawn from connected travelers, vehicles, and infrastructure. The three applications are dynamic speed harmonization (SPD-HARM), queue warning (Q-WARN), and cooperative adaptive cruise control (CACC), all of which constitute the USDOT Intelligent Network Flow Optimization (INFLO) program. The INFLO SPD-HARM application concept aims to maximize throughput and reduce crashes by generating appropriate target speed recommendation strategies for upstream traffic. Thus, it basically extends the VSL based on infrastructure detector data to applications that utilize Connected Vehicle technologies.

As the transportation community continues to develop advanced strategies to alleviate congestion, simulation models are expected to play a major role in assessing emerging ATDM strategies such as VSL and Connected Vehicle applications. However, without effective calibration, there is no assurance that the model's outputs are reliable. Several documents and results from research are available to provide guidelines for simulation model calibration and validation. However, these guidelines need to be 
re-examined and possibly revised when considering complex ATDM and Connected Vehicle strategies.

It is important to investigate the influence of the utilization of VSL/speed harmonization on traffic operations, both with and without a Connected Vehicle component. Microscopic simulation modeling will play an important role in this investigation, taking into consideration the limitations of the existing real-world applications of these strategies.

\subsection{Problem Statement}

Congestion can be categorized in two groups: recurrent congestion and non-recurrent congestion. Recurrent congestion mainly appears at bottlenecks during peak hours when too many vehicles attempt to use a common roadway with limited capacity. In order to find an alternative strategy to improve the efficiency of the existing freeway system, bottlenecks should be carefully studied, modeled and analyzed, as they are a primary reason for traffic congestion.

Bottlenecks are sections of the freeway that either have capacities less than or a demand greater than other sections during peak periods. When demand approaches or exceeds the bottleneck's capacity, breakdown will occur, which reduces a freeway's maximum flow throughput. The term "breakdown" of flow on a freeway is used to describe conditions that transition from under-saturated to over-saturated, or congested.. After breakdown occurs, not only are there congested operations, but the maximum flow throughput at the bottleneck is often significantly lower than that of the maximum capacity observed before breakdown. Recurrent freeway bottlenecks may be caused by 
on-ramp demand, lane drop, low posted speed limits, and/or spillback from off-ramps, among other reasons. Congestion is usually expressed as a stop-and-go operation, but more generally, it is experienced as a slow-and-go operating condition. VSL is among the strategies proposed to reduce the impacts of breakdown.

VSL strategies identify and disseminate the appropriate speed limits based on prevailing traffic conditions, road surface conditions, and weather conditions. In addition to the safety applications of these strategies, VSL strategies are recommended at freeway locations, especially upstream of bottlenecks with recurring congestion, to maximize the traffic throughput by delaying breakdown formation, as well as to dampen the shockwave produced once congestion starts. Several issues have been identified with infrastructure detector data-based VSL, including the need to optimize the associate parameters and sign locations, and concerns about the levels of driver compliance.

The initial documentation from INFLO pointed out that the current speed harmonization implementations are fundamentally limited by their exclusive reliance upon infrastructure-based detection and information dissemination. The introduction of Connected Vehicles technology and associated Vehicle-to-Infrastructure communication will provide a basis to detect individual vehicle trajectories that can be used as high-precision and detailed input data. Detailed traffic data can provide a better understanding of traffic conditions and driver behavior. In addition, obtaining sufficient and precise data will enable VSL algorithms to produce accurate and targeted speed recommendations by location, and also create time recommendations. Since the field Connected Vehicle data are not available, Vehicle-to-Infrastructure communication 
should be modeled in the microscopic simulation to obtain and study individual vehicle trajectories.

The above discussion indicates that VSL strategies have the potential to reduce the impacts of critical bottlenecks. However, there are still many questions to be answered before real-world implementations of this strategy can be put into effect. Simulation analysis can help answer these questions; however, there may be a need for additional calibration steps to improve its ability to model the proposed advanced strategies.

\subsection{Research Goal and Objectives}

The goal of this dissertation is to develop and assess VSL strategies to improve the operations and mobility at critical bottlenecks. The effort will include developing algorithms and methods for selecting optimal speed limits to maximize traffic operation improvements due to VSL implementations. The research will assess the utilization of both Connected Vehicle technology and infrastructure devices to support the developed VSL strategies. The specific objectives of this research are as follows:

1) Review the existing microscopic simulation calibration procedures, VSL strategies, and Connected Vehicle applications.

2) Provide a systematic calibration and validation procedure of traffic simulation models that consider traffic flow breakdown parameters, in addition to those currently used in calibrating traffic simulation models. 
3) Develop VSL strategies based on infrastructure detector data and based on Connected Vehicle data, and assess their effectiveness in improving congestion and breakdown conditions at bottlenecks.

4) Assess VSL strategies’ effectiveness under different compliance rates.

\subsection{Dissertation Organization}

This dissertation is organized into six chapters. Chapter 1 introduces the research background, describes the problems to be solved, and sets the goal and objectives to be achieved.

Chapter 2 presents an extensive literature review of breakdown characteristics, traffic micro-simulation calibration, VSL strategies implemented and previously researched and/or their outcomes, evaluation of VSL strategies, and related applications of Connected Vehicles.

Chapter 3 includes three sections. First, it describes the framework of the proposed calibration approach for microscopic simulation that considers traffic flow breakdown parameters, in addition to those currently used. Then, it discusses the background of the developed VSL strategy based on infrastructure detector data. Finally,

it presents the applications of Connected Vehicle technologies to the proposed VSL system.

Chapter 4 delineates the case study, which involves a 12-mile segment of the I-95 northbound freeway facility in Miami, Florida, to examine the products of this research. The data acquisition and preprocessing effort for this research is also described in this chapter. 
Chapter 5 explains the calibrated model's characteristics. Then, it presents the results of applying the developed VSL strategy based on infrastructure detector data. Finally, it describes the results of applying the developed VSL strategy based on Connected Vehicle data.

Chapter 6 summarizes the main contributions, draws conclusions, and offers recommendations and suggestions for future research. 


\section{CHAPTER 2}

\section{LITERATURE REVIEW}

This section first introduces freeway breakdown concepts and related literature on the subject. Then, it presents a detailed review of the current practices in micro-simulation calibration. Next, VSL algorithms, strategies, and their applications in the real world are reviewed. Finally, the Connected Vehicle technology and applications are introduced.

\subsection{Breakdown}

A freeway bottleneck location is identified by traffic congestion upstream and freely flowing traffic downstream. The term "breakdown” of flow on a freeway is used to describe the transition from speeds in the vicinity of the posted speed limit to congestion. Once a breakdown occurs, the maximum throughput can drop by 5-10\%. Papageorgiou et al. (1998) have shown that a capacity drop of $5 \%$ can increase the travel time by $20 \%$. However, this could be higher or lower depending on the ratio of the demand to the capacity of the freeway.

\subsubsection{Breakdown Definition}

The term "breakdown" has been defined in various ways by a number of researchers. These definitions are based on the amount of speed reduction or based on the average speed during breakdown. Following are some of these definitions:

- Elefteriadou et al. (2011) defined breakdown to have occurred when the speed drops below $10 \mathrm{mph}$ for at least 5 minutes. 
- Graves et al. (1998) defined breakdown to have occurred if the speed at a location is less than $30 \mathrm{mph}$ during five consecutive one-minute intervals. Whenever the speed exceeds $30 \mathrm{mph}$ for five consecutive one-minute intervals, the breakdown event is considered to have ended.

- Persaud et al. (1998 and 2001) defined breakdown as having occurred if the flow and speed drop suddenly at a location immediately downstream of a ramp, for a duration of at least five minutes.

- Okamura et al. (2000) defined breakdown to have occurred if the speeds are lower than $25 \mathrm{mph}$ or the queue exceeds 0.62 mile, for a duration of at least 15 minutes.

- Brilon (2005) defined breakdown occurrence when a short time interval experiences a sharp speed reduction below the threshold of $43.5 \mathrm{mph}$. The amount of speed reduction should be more than $6.22 \mathrm{mph}$ to be considered a sharp speed reduction. The short time interval was selected to be a one-minute interval; however, due to unavailability of data, a five-minute interval data was used.

- Kuhne et al. (2006) defined breakdown to have occurred when traffic flow is greater than $1000 \mathrm{veh} / \mathrm{hr} / \mathrm{ln}$, with a sharp speed reduction below the threshold of $46.5 \mathrm{mph}$. The amount of speed reduction should be more than $10 \mathrm{mph}$ to be considered a sharp speed reduction.

- The Manual on Uniform Traffic Control Devices (MUTCD) (2000) defines breakdown occurrence as the condition when 15-minute interval speeds are less than $40 \mathrm{mph}$. 


\subsubsection{Causes and Process of Breakdown}

Finding the main cause of breakdown is the first step to control and mitigate breakdown. For this reason, the causes of breakdown have been a topic of increased interest among researchers.

Buckley and Yagar (1974) discussed breakdown occurrence at an entrance ramp or lane drop, which they termed “capacity funnels.” At a capacity funnel, drivers merge into minimal gaps in the adjacent lane. To reach a more acceptable distance headway in this adjacent lane, drivers attempt to increase the headway by slowing down. As a consequence, drivers upstream decelerate, causing a shockwave that moves upstream.

Banks (1991) analyzed four bottlenecks using detector data and video surveillance. In three of these bottlenecks, the breakdown began with queue formation behind slower moving vehicles. As flows and densities increase, the lane change maneuver is prohibited. Eventually speeds of the platoons became unstable, resulting in sharp speed reduction. In the fourth case, breakdowns appeared both upstream and downstream of a divergence point. It was further noted that the merge and divergence rates during the breakdown were far greater than the typical capacity values.

Gazis and Herman (1992) described the development of moving bottlenecks, which are caused by slow-moving vehicles. Their discussion of breakdown on a two-lane freeway described how lane-changing vehicles that overtake slow vehicles interfere with traffic in the other lane, resulting in traffic creating a shockwave in this lane.

Elefteriadou et al. (1995) evaluated two bottlenecks using video surveillance. Analyzed data showed that the presence of vehicle clusters indicates that a breakdown may occur. 
Daganzo et al. (1999) presented a model that recognized that when one of the vehicles in the platoon wants to allow another vehicle to merge, it will slow down. Consequently, all of the cars in the platoon slow down, which causes instabilities, ultimately leading to congestion.

Daganzo (2002) categorized drivers as two types: fast-moving and slow-moving. At freeway ramp merge locations, fast-moving vehicles stay in the passing lane with short headways, while on-ramp vehicles enter and stay in the shoulder lane. Eventually, fast-moving vehicles that entered from the on-ramp try to leave the shoulder lane and merge into the passing lane, which increases the passing lane's flow. When the mainline and/or the merging flows are high, the passing lane becomes saturated, and a congestion shockwave will move further upstream. Consequently, the fast-moving vehicles try to move into the shoulder lane before merging, since the passing lane speed is now lower. As a result, the queue on the passing lane eventually spills over onto the shoulder lane.

\subsubsection{Identification of Bottleneck Location}

Identifying the locations of traffic bottlenecks is an important part of highway management. There are several methods for identifying bottlenecks. Existing bottlenecks need to be identified from historical and current field measurements based on the aforementioned breakdown definitions. A bottleneck location is likely to receive more demand than the available capacity for a period of time. The approximate location of a bottleneck is identified as the section between a detector location with the most congestion and its neighbor detector (with no congestion). For example, as mentioned earlier, previous studies considered merge points as possible bottleneck locations. 
Cassidy and Bertini (1999) reported that examined bottlenecks occurred at fixed locations, approximately 0.62 mile downstream of on-ramps. They analyzed two bottleneck locations (metered and non-metered ramps) using detector data for this purpose.

Chen et al. (2004) used an instrumented floating car method to find the locations of bottlenecks. It was mentioned that extensive data logging, as well as multiple days of data, are needed in order to remove non-recurrent bottlenecks. This approach is not sensitive to demand levels and may not be accurate enough due to limited runs and the stochastic nature of traffic varying on a daily basis.

More commonly, bottleneck locations are identified using archived detector data. Cassidy and Bertini (1999) used 30-second data to construct curves of cumulative vehicle counts and occupancy to observe the changes from free-flow conditions to queued conditions.

According to FHWA simulation guidelines (Dowling et al., 2004), a visual audit can be used as the primary method for finding bottleneck locations. Speed-distance contour plots that use detector data identify bottleneck locations. The use of multiple day data is used to ensure that a bottleneck is a recurring bottleneck.

\subsubsection{Breakdown Characteristics}

Aside from its primary causes, other characteristics define breakdown, such as duration of breakdown, average speed during breakdown, maximum pre-breakdown volume, and queue discharge. The queue discharge rate is defined as the long-run average of flow over the breakdown period. Maximum pre-breakdown flow is measured at different intervals, such as one-minute, five minutes, or fifteen minutes immediately 
before the breakdown occurs. These characteristics are important because they define capacity.

While the HCM calculates capacity based on the geometric conditions of the facility and treats it as a deterministic value, there is a significant amount of recent literature that suggests using other measures to estimate capacity, such as maximum flow before breakdown and queue discharge rate to measure capacity in the field to account for site specifications.

In addition to these characteristics, it was observed that at the same bottleneck location and for the same combinations of ramp and freeway flows, breakdown may either occur at different times or may not occur at all. This phenomenon has gained a great amount of interest and attention among researchers in two specific areas. First, researchers have come to recognize the stochastic nature of capacity and breakdown. There is still an ongoing question about which value of flow rate, either the maximum pre-breakdown flow rate or discharge flow rate, should be considered capacity for different applications. If capacity is a random variable, then what percentile of the distribution should be used as the descriptive statistic?

Elefteriadou et al. (2003) studied two major bottlenecks over a 20-day period and concluded that pre-breakdown flow and queue discharge rate are distributed normally, and that the range can be several hundred veh/hr. Geistefeldt (2008) suggested that the capacity design value should be defined as a specific percentile of the breakdown probability distribution. Another issue is identifying the breakdown probability model for use when considering breakdown. For instance, Elefteriadou et al. (1995) developed a probabilistic model for a specific on-ramp merge bottleneck. The model estimates the 
breakdown probability based on the occurrence of ramp-vehicle clusters. Kondyli (2009) suggested that lane change measures affect the breakdown probability, and driver lane-changing behaviors have a significant effect on breakdown. She developed a breakdown probability model based on this finding.

One of the concerns in studying and analyzing breakdown characteristics is the noise in traffic data. The most common way to overcome noise in the data is to aggregate traffic data over a certain time period (Ban et al., 2007; Chen et al., 2004). Another method is to use oblique cumulative curves. This method has been used specifically to study bottlenecks and find the start and end times of breakdown (Cassidy and Bertini, 1999; Muñoz and Daganzo, 2003; Sarvi et al., 2007). Cumulative curves are effective in suppressing noise; however, changes in traffic patterns are not apparent. This is the reason in the aforementioned studies that such curves were plotted with an oblique time axis to magnify the changes in traffic conditions. In these curves, the identification of changes in traffic conditions, such as the starting time of breakdown, is based on the sudden decrease in the slope of the curve. Preserving the original time resolution is one of the advantages of this method. However, this method requires adjusting the degree of the oblique axis for different situations, such as different locations and different demands, which makes this method difficult to apply. Muñoz and Daganzo (2003) used an empirical fundamental diagram (FD) to identify the start and end times of breakdown. Zheng et al. (2011) proposed a wavelet transform method that identifies the location of bottlenecks, starting time of congestion to upstream locations, and the start and end of breakdown occurrence. 


\subsubsection{Congestion Propagation}

An important issue to investigate is the congestion propagation once breakdown has occurred. Shockwave analysis is used for this purpose. A shockwave describes the boundary between two traffic states that are characterized by different densities, speeds, and/or flows. Previous studies have based the calculation of shockwave speeds on the flow-density relationship. The shockwave speed is estimated as the difference of flow over the difference of density between the conditions upstream and downstream of the bottleneck.

Also, there is a great amount of empirical case studies on finding shockwave speed. With the availability of detector data, waves can be measured by comparing the speed or occupancy time series between adjacent detector stations. The detection of these waves, however, is not always accurate, considering the amount of noise in the detector data and the fact that point detectors are normally installed at 0.5 -mile intervals. The literature is not consistent in terms of the range of values for shockwave speeds (Eleftradiu et al., 2009). Kerner (1998) suggested that the shockwave speed differs for various roadway and weather conditions. Other literature reported that shockwave speeds at bottlenecks on Japanese urban expressways range from 11 to $12.5 \mathrm{mph}$ (Koshi et al., 1983), and from 10.5 to 15 mph (Iwasaki, 1991). Lu and Skabardonis (2007) examined the vehicle trajectory datasets collected as part of the FHWA NGSIM program at two freeways, and found an average congestion propagation speed of $11.4 \mathrm{mph}$. They also found that this speed is independent of the speed prior to congestion. 


\subsection{Calibration}

Traffic simulation is widely used and increasingly applied for the assessment of the performance of transportation systems, traffic operations, and management alternatives. Simulation is cost-effective, allows risk-free assessment, and provides an efficient assessment approach. However, without calibration, there is no assurance that the model's outputs are reliable and that the model will correctly predict traffic performance. Calibration is the adjustment of model parameters to improve the model's ability to reproduce local traffic conditions. To show the importance of calibration, Bloomberg et al. (2003) showed that a difference of $13 \%$ in freeway speeds between real-world and simulation estimates for existing conditions can produce a difference of $69 \%$ in the forecasted freeway speeds for future conditions.

\subsubsection{Trial-and-error Methods}

Trial-and-error methods tend to be more frequently used in practice than other methods, as they are generally less complex, and when performed by experienced modelers, can produce good results. The trial-and-error methods involve an iterative adjustment process. This process continues until both precision requirements and performance target are met. This method is simple and easy to apply, but the choice of the feasible range often relies on the analyst's modeling experience and judgment to make a good choice. Chu and Liu (2004) developed a four-step trial-and-error-based approach that includes the calibration of driver behavior models, route choice, origin-destination estimation and model fine tuning. Dowling et al. (2002) developed another four-step trial-and-error method to calibrate a model. These four steps include 
error checking, calibration for capacity, calibration for demand, and overall analysis of performance.

Park and Schneeberger (2003) proposed a nine-step calibration procedure. The three main components of the procedure are: 1) calibration component setup (data collection, selection of calibration parameters and MOEs); 2) calibration effort; and 3) evaluation and validation of the calibrated model. A case study corridor that was modeled in the VISSIM model was calibrated using this procedure. They used the results from the t-test to compare the simulation and field travel time means as the criterion to determine when a model is adequately calibrated. The case study was only based on a single day of data collection and generated the parameter sets from a linear regression model, thus, it did not account for the day-to-day variability of traffic conditions.

Hourdakis et al. (2003) proposed a four-step calibration and validation procedure that includes: 1) volume-based calibration; 2) speed-based calibration; 3) capacity-based calibration; and 4) validation. In each step, a quasi-Newton algorithm was used to find local optimum parameters and, in all four steps, Theil's inequality was used as a goodness-of-fit measure. The proposed procedure was applied to a 12-mile long freeway network modeled using AIMSUN.

Dowling et al. (2004) introduced a top-down calibration approach, which consists of a three-step calibration process that involves capacity as measured by queue discharge rate, and system performance calibration. Capacity calibration is very important, as it has a significant effect on the predicted system performance. The authors' recommendations include first focusing on changing network-wide parameters, and then changing link-specific parameters. 
Gomes et al. (2004) used three speed contour maps, corresponding to a heavy, typical, and light day of traffic to identify field bottlenecks. The calibration objective was to match the locations of the bottlenecks, bottleneck start times, queue lengths, and time durations. However, the study did not match flow data because of the large variations identified in traffic flow. In addition, no quantitative measures were developed.

Zhang and Owen (2004) proposed a procedure that includes quantitative and statistical analyses at both the macroscopic and microscopic levels, as well as animation comparison. The performance measures used in this procedure were the average speed and traffic volume at the macroscopic level and the vehicle trajectory plot and headway distributions at the microscopic level. The animation comparison was conducted as a validation procedure. Based on Zhang and Owen's study, some of the advanced micro-simulation traffic models such as CORSIM and VISSIM are using multi-regime simulation logic. For example, car-following regimes in these models can be normal or uncomfortable. The uncomfortable regime is defined as the model allowing the distance between successive vehicles to be arbitrarily close when speeds are identical.

Zhang et al. (2004) identified the parameters in the CORSIM simulation model that can affect the assessed capacity in the simulation. The analysis was based on investigating the impact of one parameter at a time on the selected MOEs. The results showed that the car-following sensitivity multiplier and the mean free-flow speed greatly affect the MOEs. The Pitt car-following constant, lag acceleration/deceleration time, and time to complete lane change had a medium effect. The rest of the car-following and lane-changing parameters did not have significant effects on the MOEs. 
Ban et al. (2007) introduced a three-step approach for bottleneck calibration. The first step is the visual assessment of the speed contour maps from simulation versus real-world data. Ban et al. also used binary speed contour maps, where each cell is 1 if it is congested; otherwise, it is " 0. ” The second step consists of matching the binary speed contour maps from simulation against real-world data. The last step is a detailed speed calibration.

Halkias et al. (2007) simulated a highway in Athens, Greece in order to assess bottleneck mitigation strategies. The queue lengths and speed values were the parameters considered for comparison between field measurements and simulation results. For further alternative analysis, the volume was increased by $20 \%$ to make sure that hidden demands had been considered and more severe downstream bottlenecks would not occur. Halkias et al. recommended that a wider perspective of freeway analysis is required, in addition to focusing on the bottleneck area to make sure that the investigated scenario will not lead to new bottlenecks downstream.

Zhane et al. (2008) categorized calibration approaches into two groups; the most popular group is the flow profile approach, which compares the simulation results against the field observations for every interval. The other approach is the fundamental diagram approach, which is based on capacity and the shape of the flow-occupancy diagram. This approach focuses on replicating field-observed capacities.

Most often, micro-simulation models are calibrated using data from a single time period and may fail to adequately represent traffic conditions outside of that specific time period. Rakha (1998) conducted a two-factor analysis of variance (ANOVA) to gain a better understanding of the stochastic nature of traffic conditions. This approach requires 
comparing the results from a set of simulation runs with field data observed across different days. The least-squares error (LSE), least Poisson error (LPE), and visual inspection were used to measure the variability in link flows. Comparing the flow between days shows that the LSE varies from 1.7 to 3.6 percent of the mean flow, and the LPE was found to vary from 3.2 to 5.2. A graph was used for visual inspection, in which the data points were scattered around the line of unbiased correlation ( $45^{\circ}$ line). The authors recommended that all of these measures should be considered with each other since in some cases, the error estimates do not coincide with the visual inspection.

Henclewood and Fujimoto (2012) investigated the calibration of a model for two different periods, focusing on ten effective parameters. For this purpose, 1,000 different parameter sets produced by a Monte Carlo simulation were used as inputs to VISSIM. Out of the 1,000 sets, there were 93 well-calibrated models for the first time period, and 34 well-calibrated models for the second time period. Only one parameter set was found to be sufficiently calibrated for both periods, based on travel time and saturation flow rates. They concluded that the calibration parameters should be allowed to change with respect to time to account for the changes in driving behavior and environment.

\subsubsection{Heuristics-based Methods}

One of the widely attempted approaches in micro-simulation model calibration is the use of the Genetic Algorithm (GA) in the calibration process. Three reasons that researchers frequently use to justify the choice of genetic algorithms are: 1) it does not need gradient information, which is usually not available due to the complex format of micro-simulation; 2) it avoids exhaustive enumeration, which can save significant 
computational time; and 3) it always maintains a set of feasible solutions before reaching on optimum answer. In general, GA-based approaches consist of two primary components: 1) feasibility test, and 2) GA-based optimization. First, it is important to identify key parameters affect the results. This could be done using different approaches such as the ANOVA or other statistical plots and visual observations. The feasibility test is used to determine whether or not the set of calibration parameter ranges are feasible. This step should be repeated until the feasibility test is satisfied by adjusting the range of parameters, which could be implemented using the Latin Hypercube Sampling (LHS). This algorithm is used to reduce the number of combinations to a reasonable level, while still covering the entire parameter surface. It is well known that conducting the GA optimization requires large running times, compared to other optimization techniques, while often ensuring better solutions than other methods.

Schultz and Rilett (2004) analyzed the effects of the car-following sensitivity factors distribution on CORSIM results. Two alternatives were first considered: random distribution, where each factor is an independent parameter, and one distribution, in which all factors are generated from a distribution of measures of central tendency and dispersion. Schultz and Rilett chose to focus their study on the second alternative, which only requires two parameters (mean and variance), which simplifies the process. Based on previous studies, two possible headway distributions - the normal and log-normal distributions, were selected to generate the car-following sensitivity factors. Using the GA approach, Schultz and Rilett calibrated a simulation model for the IH-10 in Houston, Texas, for the AM and PM peak periods. Both proposed distributions produced better results, compared to the default distributions for both time periods. The results show that 
the optimal distribution for the AM peak is different than the PM peak. In addition, the log-normal distribution produced slightly better results.

Kim and Rilett (2004) used a GA method to calibrate a CORSIM micro-simulation model for two corridor systems in Texas. Their study considered 19 parameters in CORSIM that consisted of 11 car-following sensitivity parameters, 2 acceleration/deceleration parameters, and 6 lane-changing parameters. They implemented the binary coding method to code the 19-parameter set into a 121-bit binary string as an individual's chromosome in the GA. The large search space, described above, illustrates the importance of using an efficient optimization method.

Park and Qi (2005) developed a GA-based procedure for calibrating the VISSIM micro-simulation model. They used the Latin Hypercube Design (LHD) to reduce the number of possible combinations of parameter values. Their calibration approach was tested using two case studies, including an isolated signal intersection and a highway segment with work zone. Travel time was considered the performance measure for both calibration and validation. Their approach reached the optimal solution after 10 generations in the GA optimization.

Lee et al. (2013) introduced a simplified procedure for calibration. Since their previous study in implementing a GA simulation calibration was not practical, it was not widely used by traffic engineers. Once all samples were evaluated using the LHS approach, the solution with the most promising fitness values were chosen. Case studies on urban signalized corridor and freeway section show that this procedure outperforms the previously used GA-based procedure. 
Ma et al. (2007) proposed a simultaneous perturbation stochastic approximation (SPSA) method-based calibration approach and used it to calibrate a system in PARAMICS. They compared the performance of their approach against other heuristic methods, such as the GA and the trial-and-error iterative adjustment algorithm. This comparison was done by measuring the computation time, which showed that their method outperforms the other two heuristic methods.

Lee and Ozbay (2009) proposed a Bayesian sampling approach in conjunction with the application of the SPSA optimization method. The Bayesian sampling technique was used to create unbiased initial input data covering the entire search space. Lee and Ozbay compared their approach to the standard SPSA-based approach, and the results showed that their approach requires less computation time. It is interesting to point out that based on their literature review, most of the previous studies failed to note that having the same mean between the observed data and simulation output does not imply that these distributions are identical. In validating the model, Lee and Ozbay compared its outputs with the distribution of the observed values using the Kolmogorov-Smirnov test to handle day-to-day traffic variations.

Paz et al. (2012) introduced a calibration procedure based on the SPSA algorithm in order to calibrate all of the parameters simultaneously. This method is an iterative approach that uses gradient estimations of the objective function to determine an optimal solution.

Fellendorf (2001) used the simulated annealing optimization method to calibrate a roundabout modeled in VISSIM micro-simulation. Queue positions were considered 
measurements of effectiveness. Fellendorf reported that calibration results were promising.

Menneni et al. (2008) introduced a calibration methodology based on an evolutionary optimization algorithm that uses the speed-flow relationship as a calibration objective to address the stochastic nature of capacity. They stated that instead of using a single numerical value, the distribution of capacity values should be used. Using a distribution allows for the use of queue discharge flow and pre-queue flows, which can be derived from the speed-flow graphs. Menneni et al. claimed that this approach can replicate the whole range of traffic behaviors since the speed-flow graphs provide information on all three regions: free-flow, congestion, and discharge. They concluded that the results from this approach outperform the results from the calibration based on the objective functions that include the maximum 5-minute flow and maximum 5-minute flow sustained for 15 minutes.

Hollander and Liu (2008) presented a rather comprehensive review of many of the current calibration methods, while attempting to highlight the fundamental requirements for calibrating microscopic simulation models. They provided a tabulated summary on the methods researchers used to calibrate different micro-simulation models and their stopping criteria to indicate that the calibration results are suitable. In examining these criteria, one may notice that many tend to be subjective due to their dependence on what is being modeled and the goals of the modeling effort.

In summary, traffic simulation models have been widely and increasingly applied for the assessment of transportation systems, traffic operations, and management alternatives because simulation is cost cost-effective, allows for a risk-free assessment, 
and provides an efficient assessment approach. However, without calibration, there is no assurance that the model's outputs are reliable or that the model will correctly predict the traffic performance expected in the real world. Calibration is the adjustment of model parameters to improve the model's ability to reproduce local traffic conditions.

\subsection{Variable Speed Limit}

Traffic congestion is a critical social issue that is encountered on a daily basis. It appears in the peak hour when too many vehicles attempt to use a common roadway with limited capacity. It is a source of productivity and efficiency loss, fuel wastage, and excessive air pollution. The areas that mostly suffer from these problems are large cities and freeways. Expanding road infrastructure is one of the solutions, but often is constrained by the limited availability of right-of-way and capital investments. More efficient use of existing road networks is a promising solution that transportation practitioners are examining. Therefore, there is a tremendous need to understand the effects of different dynamic control methods on freeway operations, as well as to identify cost-effective control strategies to address identified issues with operations. Advanced Traffic and Demand Management (ATDM) strategies, such as ramp metering, variable speed limits, and Connected Vehicles, are among the methods that are increasingly being considered to improve the efficiency of existing freeway systems.

Static speed limits are designed to provide motorists with safe driving speeds. While these safe speeds are effective during ideal conditions, they fail to provide recommended safe speeds during adverse weather or congested driving conditions (Sisiopiku 2001). Variable Speed Limit (VSL) systems dynamically adjust the speed limit 
based on the prevailing traffic condition, road surface condition, and weather condition information. Such strategies are used to deal with congestion, incidents, weather and/or special events by reducing congestion impacts and crash risk. Infrastructure-based dynamic message signs are used to disseminate the VSL to drivers, although in-vehicle information devices can also be used.

Over time, two general applications have evolved in the use of speed limits. The first emphasizes the safety benefits of VSL, such as reducing the number of rear-end collisions and traffic homogenization (Harbord, 1995); whereas the second is more focused on avoiding or mitigating traffic flow breakdown by reducing the input flow at bottlenecks using VSL (Lenz et al. 1999). For this second type of application, the VSL signs are installed upstream of the bottlenecks, with recurring congestion as a way to reduce the speed of the congestion build-up shockwave produced once congestion starts.

A theoretical study by Kohler (1974) showed that when the headways in a chain of vehicles are below a certain bound, the traffic becomes unstable. The inhomogeneities in the traffic stream readily lead to the small disturbances needed for congestion to set in. Inhomogeneities can be raised from speed differences between consecutive vehicles in one lane, speed differences among lanes, or flow differences among lanes. Through the use of VSL control, traffic planners hope to achieve a more uniform distribution of traffic density over freeway links, thereby preventing the high traffic density that leads to traffic breakdown.

This section first summarizes the known the effects of implementation of VSLs in the real world. Next, it provides an overview of the evaluation of VSL algorithms 
conducted in past research using simulation. Finally, it summarizes driver behaviors around VSLs, as reported in previous studies.

\subsubsection{Implementation of VSLS}

VSL systems have been implemented to control speed in some locations throughout the UK, Netherlands, USA, Germany, Australia, and New Zealand. Currently, there is a very limited amount of documentation describing the quantitative mobility and operational impacts. In fact, mobility-related benefits have been derived mostly from the use of simulation. However, safety benefits were documented for several of the systems based on real-world data.

Most of the VSL systems were implemented as safety countermeasures to address adverse weather conditions. In Tennessee, a VSL system was implemented in 1993 along a 19-mile freeway segment on I-75 utilizing 10 signs. The goal was to reduce the occurrence of crashes due to visibility reduction during adverse weather conditions, especially fog. The posted speed limits and messages were automatically selected based on data collected using environmental sensor and vehicle detectors. The system had the ability to close down the entire stretch of roadway during severe fog conditions and divert traffic. There were no crashes due to fog after the system was implemented (Robinson, 2000).

In Arizona, a VSL system based on a fuzzy control algorithm was implemented in 1998 along I-40 in order to find appropriate speeds for different weather conditions and road surface conditions (Robinson, 2000). 
In Washington, a VSL system was implemented in 1997 (and is still active) on I-90 across the Snoqualmie Pass. The goal was to improve safety and inform motorists of road conditions and weather information. It was found that VSLs may lose their effectiveness without enforcement by the State Patrol, and that VSLs reduce the mean speed and increase the speed standard deviation (CTC and Associates LLC (2003), Steel et al., 2005).

In the Netherlands, a VSL system was installed in 1991 along the A16 motorway on a 7.4-mile segment utilizing 16 signs. The goal was to improve safety during fog conditions. The posted speed limit was automatically switched and selected based on the visibility and crash occurrence. The normal posted speed limit was $62 \mathrm{mph}$, and if the visibility dropped below 460 feet, the posted speed limit would be reduced to $50 \mathrm{mph}$; and for visibility below 230 feet, the posted speed limit would be $37 \mathrm{mph}$. Furthermore, when an incident was detected, a speed limit of $31 \mathrm{mph}$ was posted on the first sign upstream of the incident, and $43 \mathrm{mph}$ on the second sign upstream of the incident (Robinson, 2000). Zarean et al. (1999) evaluated this system and showed that drivers reduced their mean speeds by about 5-6 mph during fog conditions.

Rämä (1999) conducted a more detailed study on weather-controlled speed limits and signs. The study looked at two scenarios, which were compared with a control case: one in the summer where the maximum speed limit was $75 \mathrm{mph}$, and one in the winter where the maximum speed limit was $62 \mathrm{mph}$. The control cases were the normal operating procedures in the summer and winter months. In the winter, during adverse road conditions, the speed limit was lowered to $50 \mathrm{mph}$. A $2.1 \mathrm{mph}$ decrease in speeds was observed. It was noted that during adverse conditions, when it is harder for drivers to 
observe VSL signs that affect driver visibility, the VSL was very effective in reducing the speeds, compared to the control case. The study showed that the VSLs decreased the mean speed and standard deviation of speeds.

Several VSL systems were implemented to smooth flow and reduce congestion-related crashes. A study of European VSL implementations shows that VSLs can stabilize traffic flow in congestion and thus decrease the probability of crashes. However, some of the cases in the United States show that the VSL system failed to improve mobility. The first variable speed limit system in the United States was implemented on a 3.2-mile freeway segment of the M-10 in Detroit, Michigan in 1960, with 21 VSL sign locations. The system was designed to alert motorists to slow down when approaching congestion, and accelerate when leaving a congested area. The posted speed limits were manually switched and selected by the operator based on CCTV and plots of freeway speeds. The posted speed limits were allowed to vary between $20 \mathrm{mph}$ and $60 \mathrm{mph}$, with an increment of $5 \mathrm{mph}$. The evaluation results showed that the VSL system failed to improve the situation and had no effect on vehicle speeds. The system was terminated sometime after 1967 (Robinson, 2000).

In New Jersey, a VSL system was implemented in the 1960s along the New Jersey Turnpike, over a 148-mile freeway segment utilizing 120 signs. The goal was to reduce speed limits during congested conditions. Later, the system became part of an ITS system that improves safety and mobility by warning drivers of lane closures and crashes. The posted speed limits were automatically switched and selected based on the average travel speeds. The posted speed limits were allowed, varying between $30 \mathrm{mph}$ and the normal posted speed limit $(65 \mathrm{mph}, 55 \mathrm{mph}$, or $50 \mathrm{mph}$, depending on the freeway 
segment), with an increment of $5 \mathrm{mph}$. The main six conditions that caused the switching of the posted speed limits were: vehicle collisions, traffic congestion, construction, icy road conditions, snowfall, and fog. Based on the Turnpike Authority observation, the system’s performance was satisfactory. It was noted, however, that the system needed enforcement by the police (CTC and Associates LLC, 2003; Steel et al., 2005).

In Florida, a VSL system was implemented in 2008 along a 9-mile portion of I-4. The goal is to enhance safety during congestion. Traffic conditions are classified as either free-flow, light congestion, or heavy congestion, based on occupancy. The posted speed limits are supposed to be $30 \mathrm{mph}$ for heavy congestion (occupancy less than 16\%), 40 mph for light congestion (occupancy between 16\%-28\%), and the normal speed limit, 50 mph for free-flow (occupancy greater than 28\%). The speed limits were automatically selected every 120 seconds. Each sign is linked to two or three downstream detectors, and the occupancy value is averaged between them. The system also ensures that the posted speed limit does not change by more than $10 \mathrm{mph}$ between two adjacent sets of VSL signs (Haas et al., 2009). In an evaluation study, it was determined that since vehicles were not complying with the reduced speed limits, the VSL system was not effective (PBS\&J, 2009).

In England, a VSL system was implemented in 1995 on the M25 motorway on a 14-mile segment with 23 VSL signs. The goal was to smooth traffic flow by reducing stop-start driving in order to respond to congestion. The posted speed limits were decreased from $70 \mathrm{mph}$ to $60 \mathrm{mph}$ when the volume exceeded 1,650 vehicles per hour per lane (veh/hr/lane), and further lowered to $50 \mathrm{mph}$ when volume exceeded 2,050 veh/hr/lane. Each VSL sign is linked to a downstream detector location. Evaluation of the 
results showed that the traffic accidents decreased by $10-15 \%$, and the compliance rate with the VSL system was very high (Robinson, 2000).

Another example of using VSL to improve safety is the implementation in Seattle, Washington in 2010. The overhead signs display individual speeds for each lane and warn of approaching lane closures and traffic congestion. The posted speed limit varies from $40 \mathrm{mph}$ to $60 \mathrm{mph}$, based on speed and volume data. The speed limit is enforced by the Washington State Patrol (Elefteriadou et al., 2012).

In Sweden, a VSL system was installed in 2004 along the E6 motorway in Mölndal. At first, the VSL was implemented as an advisory speed limit, but was later changed to an enforceable speed limit. Lind (2006) tried to determine how the VSL was perceived by motorists in both the enforceable and advisory implementations. Before the VSL system was implemented, the posted speed limit was $43 \mathrm{mph}$. The speed limit for free-flow conditions was raised to $56 \mathrm{mph}$. In dense traffic, the posted speed limit was reduced in a stepwise manner. At a traffic flow rate of $950 \mathrm{veh} / \mathrm{hr} / \mathrm{lane}$, the speed was reduced to $43 \mathrm{mph}$. It can be further reduced to 31 and $17 \mathrm{mph}$, depending on the density. Two-thirds of interviewed drivers indicated that they supported the VSL and considered reductions of queue lengths and hectic driving scenarios as benefits of the system. When the advisory speed limit was displayed, the crashes were reduced by $20 \%$, and when the enforceable speed limit was displayed, the crashes were reduced by $40 \%$. The results showed an increase in the average speed for all driving conditions, and as much as a 25 mph increase in potential queue formation scenarios.

In some cases, the VSL system focuses on special types of vehicles. In Denver, Colorado, a VSL system was implemented in 1995 along the Eisenhower Tunnel on I- 
70. The goal was to enhance truck safety by displaying vehicle-specific safe operating speeds for long downgrades. The posted speed limit for the trucks was computed automatically based on the truck's weight, speed, and axle configuration. The speed limit was advisory, and evaluation results showed that truck-related accidents declined on the steep downhill grade sections after the implementation of the VSL system, even though the truck volume increased (Robinson, 2000).

Young (2010) studied the effectiveness of VSL signs on a 100-mile stretch of I-80. The study showed that the average vehicle speeds were reduced by $0.47-0.75 \mathrm{mph}$ for every $1 \mathrm{mph}$ reduction in the posted speed limit. Lee et al. (2006) found that for highly congested locations, the VSL provided a reduction in a crash potential of $25 \%$, but it increased travel time.

Papageorgiou et al. (2008) studied the impact of VSLs on the flow-density fundamental diagram through simulation of a motorway in Europe. The posted speed was selected based on a threshold control algorithm, with possible speed limits of $60 \mathrm{mph}, 50$ mph, and $40 \mathrm{mph}$. The study showed that the $50 \mathrm{mph}$ setting resulted in the highest improvements in traffic flow. The $40 \mathrm{mph}$ setting was useful at high occupancies for safety reasons, but it did not improve the mobility. Papageorgiou et al. explained the impacts by utilizing the flow-density fundamental diagram and showing a decrease in the slope of the relationship of under-critical conditions, shift of the critical density to higher values, and higher flows at the same occupancy values in overcritical conditions.

Several studies showed that the mean speeds decrease when a VSL is implemented, indicating that the VSLs do affect the speed at which motorists drive. Several studies that showed the speed standard deviation is also expected to decrease, 
which is associated with safety benefits. Although the safety benefits of implementing the VSL control have been well-established, most of the previously developed VSL control strategies' effects on improving traffic flow efficiency and the impacts on capacity is unclear (Robinson, 2000). Based on this literature review, a very limited number of implemented VSL systems have documented improvement on traffic mobility. The VSL system called “SPECIALIST," presented by Hegyi et al. (2010), showed improvement in traffic mobility. This VSL system was implemented in order to deal with moving congestion by reducing inflow traffic to congested area. This strategy successfully resolved $77 \%$ of shockwaves, resulting in a travel-time savings of 35 vehicle-hours per shockwave.

\subsubsection{Evaluation of VSL Algorithms}

Simulation is a very valuable tool for assessing the impact of changes in the transportation system and selecting optimal alternatives without actually implementing and testing them in the field. Most of the studies on VSL, especially those that evaluated its impact on mobility and congestion, were conducted using simulation. The previously developed VSL algorithms for traffic mobility applications can be categorized into two groups: 1) reactive algorithms, which set the speed limit based on current traffic conditions; and 2) predictive algorithms, which set the speed limit based on predication modules using current traffic conditions.

Lee et al. (2004) used a crash prediction model to assess the safety effects of VSL based on the simulation model PARAMICS. With this model, three detector locations relay information to a controller that averages their values into one crash potential value. 
When crash potential exceeded the threshold, the speed limits were selected and reduced based on the average speeds. The normal speed limit is $55 \mathrm{mph}$ and will be reduced to 31 mph if the average speed is less than $37 \mathrm{mph} ; 37 \mathrm{mph}$ if the average speed is between 37 and $44 \mathrm{mph} ; 44 \mathrm{mph}$ if the average speed is between 44 and $50 \mathrm{mph}$; and $50 \mathrm{mph}$ if average speed is higher than $50 \mathrm{mph}$. The results found that the reduction in speed limits can reduce the average total crash potential, and the greatest reduction in crash potential is expected to occur at the locations with high traffic turbulence, such as at a bottleneck. However, the VSL also resulted in an increase in travel time.

Abdel-Aty et al. (2006) evaluated the safety benefits of VSL on I-4 in Orlando, Florida using PARAMICS. The algorithm reduced speeds upstream of congestion, and raised the speed limits after a congested area. This study considered low-speed and medium- to high-speed as two speed regimes. The results indicated that the VSL system has safety benefits in the medium to high-speed regions; however, for the low-speed region, which is the congested area, the system did not produce safety benefits. The results also show that changing the speed abruptly outperforms gradual speed changes. Aside from safety benefits, this algorithm also decreased the travel time, according to the simulation analysis results.

Piao et al. (2008) assessed the safety benefits of in-vehicle VSL instead of roadside VSL using the microscopic simulation model AIMSUN. VSLs were applied when the speed difference between a queuing section and the upstream section was greater than $12.5 \mathrm{mph}$. It was assumed that all vehicles were equipped with in-vehicle devices to communicate speeds and receive VSL. The posted speed limits ranged between $62 \mathrm{mph}$ and $37 \mathrm{mph}$, with a $5 \mathrm{mph}$ increment. The simulation results showed 
that the VSL reduced speed differences, small time headways, small time-to-collision events, and lane change frequency. This overall reduction creates homogenization and reduces crash potential. The authors also indicated that large speed variations could occur because some vehicles did not have the in-vehicle device.

Hegyi et al. (2003) developed and assessed a predictive model for coordination of VSLs to eliminate shockwaves at bottlenecks using the METANET simulation tool. The objective of this model was to minimize the travel time with safety constraints to prevent large speed limit fluctuations. It uses rolling horizon values to continuously update the optimal solution. The results showed that the model is successful in surpassing the shockwave, and it created a scenario with less congestion and higher outflow.

Lin et al. (2004) assessed two online algorithms for VSL controls at highway work zone operations. The first algorithm focused on minimizing the queue upstream of the work zone location by reducing approaching traffic speed so as to increase the average headway for vehicles to merge onto adjacent lanes; while the second one aimed at maximizing the throughput under some predefined safety constraints. The simulation results by CORSIM showed that the second algorithm produced more promising results than the first one in terms of reducing the speed variance, although the average speed did not change significantly.

Allaby et al. (2007) evaluated the impacts of a candidate VSL system on a 5-mile section of the Queen Elizabeth Way in Toronto, Canada using the microscopic simulator PARAMICS. A VSL control strategy was designed to reduce vehicle speeds upstream of the bottleneck to provide safer deceleration for vehicles approaching the queue and to increase the mean bottleneck speed by reducing stop-start conditions. The VSL algorithm 
was based on a decision tree that uses threshold values for flow, occupancy, and average travel speed. The base speed used was $62 \mathrm{mph}$, and it could be reduced to $50 \mathrm{mph}$ and 37 mph based on the threshold values. If the volume is less than or equal to 1600 $\mathrm{veh} / \mathrm{hr} / \mathrm{lane}$, the next step is to consider occupancy. If occupancy is less than or equal to $15 \%$, the maximum speed limit (62 mph) is posted. If the occupancy is greater than $15 \%$, the average speed determines which speed is displayed. If the volume is greater than $1600 \mathrm{veh} / \mathrm{hr} / \mathrm{lane}$, the logic skips straight to the calculation based on the average speed. Each VSL sign is linked to an adjacent loop detector, and each sign operates individually. The results of the simulation showed that the implementation of the VSL signs could significantly improve safety in higher congestion levels; however, the use of the VSL signs had negative impacts on travel time. The most desirable results were observed under moderate congestion.

Hegyi et al. (2005) expanded their original work described earlier (Hegyi et al., 2003) to modeling predictive control through coordination of VSLs and ramp metering. It was suggested that the VSLs should be used if the speed limits can limit the flow sufficiently; however, if the flow becomes too large, ramp metering should be implemented. The authors suggested that the integrated use of both technologies can produce more favorable results.

Another study on safety and mobility benefits of the integration of ramp metering and VSL was done by Abdel Aty and Dhindsa (2007) using PARAMICS. The result indicated that the VSL and ramp metering are more effective when integrated together. The best implementation strategy included speeds that were incremented by $5 \mathrm{mph}$ over a 
half mile. It was indicated that for safety improvements, the best scenario was to only increase the downstream speeds.

Ghods et al. (2009) investigated the use of ramp metering and VSL using METANET. They used an adaptive genetic-fuzzy algorithm to provide a corresponding metering rate and variable speed limits based on local speed, density and queue length of the on-ramp. Using fuzzy logic allows for input data to have partial membership to a category, as opposed to the traditional "crisp" membership or non-membership options. The study indicated that the genetic fuzzy ramp metering and VSL control improved the total time spent in the system (TTS) by $15.3 \%$.

Carlson et al. (2010) expanded the work of Papageorgiou (2008) to explore the integration of ramp metering and VSL to address potential bottleneck or high volume merging situations using the METANET simulation tool. Four scenarios were evaluated: no-control, VSL control, ramp metering, and integrated control. The study showed that when applied upstream, the VSL can act similarly to ramp metering, where the flow is held back on the mainstream rather than on the ramp. The traffic arriving at the bottleneck is temporarily reduced, and the system delays the propagation of the congestion. The VSL case decreased TTS by $15.3 \%$, and when VSLs and ramp metering were used in conjunction with each other, the TTS was reduced by as much as $19.5 \%$. They concluded that VSL can improve traffic flow and capacity by reducing the capacity drop at bottlenecks. However, at uncongested conditions, the VSL has negative impacts on mean speed and flow efficiency.

Elefteriadou et al. (2012) evaluated the use of three different VSL algorithms: the VSL algorithm presented by Allaby et al. (2007), the VSL algorithm implemented on I-4 
in Orlando, Florida, and the VSL algorithm implemented on the M25 in England. The evaluation assessed the impacts of these VSL systems on two bottlenecks on I-95 in Miami, Florida by using CORSIM. Different threshold values, as well as several different VSL sign locations, were tested. They concluded that the algorithms tested improved the mobility at bottlenecks and areas upstream of the bottleneck, and increased the throughput by a maximum of 120 to $360 \mathrm{veh} / \mathrm{hr}$. They found that the best performing algorithm and scenario is not the same for both bottleneck locations, and that the best sign location is not the same for all algorithms and scenarios. The results showed that improper selection of thresholds or sign positioning can cause negative impacts on traffic conditions; hence, an optimization-based study was recommended to obtain optimal thresholds, sign locations, and detector locations.

Talebpour et al. (2013) explored the impacts of early shockwave detection on breakdown formation and safety. They used the Allaby et al. (2007) speed limit decision tree with different thresholds. Results showed a significant improvement in traffic flow characteristics under congested conditions. The results indicate that a $10 \%$ compliance with the VSL is adequate enough to achieve the desired outcomes. The results also suggest that finding the optimal location of speed limit signs is important because it is most effective. However, the authors recommend future studies on these findings.

Many researchers used the macroscopic traffic flow model METANET to test their VSL strategies (Carlson et al., 2010). Wang (2011) compared the results of macroscopic simulation and microscopic simulation. Although macroscopic simulation showed improvement in traffic flow, microscopic simulation studies did not show the same results and demonstrated that their proposed VSL could not improve the traffic 
flow. They concluded that macroscopic simulation, because it aggregates traffic data using a generalized car-following model, failed to capture individual vehicle transitory responses and the secondary shockwaves generated by the VSL system.

\subsubsection{Driver Behavior Around VSLS}

As mentioned in the previous sections, one of the most important issues in implementing VSLs is driver behavior, and whether or not drivers will obey speed limit signs. There were a few projects, such as the I-4 project in Orlando, Florida, in which the VSL was not successful or effective because drivers were not complying with reduced speed limits (PBS\&J, 2009). The effectiveness of a VSL system is dependent on the driver's acceptance and compliance rate of the system. Increased compliance of variable speed limits can be accomplished through enforcement strategies, and by making drivers more aware of the current speed limit and the specific strategies of VSL implementation.

Tignor et al. (1999) suggested that the key to increasing compliance with VSL is automated enforcement. In England, automated enforcement improved compliance with VSL, resulting in a 5-10\% increase in capacity, and a $25-30 \%$ decrease in the number of rear-end collisions. After the initial installation of auto-enforcement cameras, it was discovered that the flash produced by the cameras was enough to make drivers obey the posted speed limit, as long as there were active cameras in a few locations. For this reason, locations of actual cameras were rotated so that drivers would never know which cameras were actually taking pictures.

Rämä (2001) studied the effect of warning messages on VSL compliance in Finland. The VSL posted two speed limits: $62 \mathrm{mph}$ during good road conditions, and 50 
mph during adverse weather conditions. It was shown that during poor weather conditions, providing a warning message with the speed limit reduced the mean speed by $1.55 \mathrm{mph}$, while the mean speed was higher if the speed limit was posted without a warning message. The author suggested that there would be more of an acceptance of VSLs if drivers knew why the speed limits were being reduced.

Brewer et al. (2006) investigated the effectiveness of several speed control devices, such as speed display trailers, DMS with radar, and orange border speed limit signs on the compliance with speed control in work zones. The results indicated that drivers will reduce their speed when their own speed is displayed. However, adding an orange border to a speed sign does not greatly increase the compliance, even though it increases the visibility of the sign. Based on data from the study, the authors concluded that drivers will travel at the speed at which they feel the most comfortable, unless they are aware of potential enforcement.

\subsection{Connected Vehicle Technology}

As stated before, road mobility significantly contributes to the society’s economy and welfare. However, the increase in the number of vehicles is creating new problems, such as longer travel times and reduced travel time reliability. To address these challenges and ensure a safe and efficient transportation system, advanced vehicle technologies are being developed by automobile manufacturers and after-market companies. These technologies can be categorized into two major groups: Assisted Driving Systems/Autonomous Vehicles and Connected Vehicle. 
This section presents a review of previous research on assisted driving systems and Connected Vehicle technologies and their applications, as related to this effort.

\subsubsection{Assisted Driving System}

Driver assistance systems are based on the idea that an on-board computer can assist drivers with a more comfortable and safer drive by using sensors and cameras connected to a central vehicle information system that recognizes potentially dangerous situations. This system provides warnings to the driver or directly intervenes in the driving process by braking or accelerating. These types of systems can be classified as the following: side assist, front assist, brake assist, blind corner monitor and parking, and rear assist. This technology could affect the flow of traffic, particularly with respect to car-following, lane-changing and gap acceptance characteristics (Elefteriadou et al., 2011).

Elefteriadou et al. (2011) evaluated assisted driving systems, focusing on two technologies, Adaptive Cruise Control (ACC) and Lane Change Assist (LCA), by using the micro-simulation software CORSIM. CORSIM was modified to model assisted driving technology. The evaluation considers different demand and market penetration scenarios.

The ACC technology allows the vehicle to decelerate when getting closer to the preceding vehicle, and accelerate again to the preset speed when traffic allows, using either a radar or laser technology setup. The driver is able to choose the desired maximum speed and the time headway derived automatically by the vehicle's equipment. The LCA also provides warnings to drivers of traffic presence at a target lane while a 
driver is changing lanes, as indicated by the driver activating a turn signal (Elefteriadou et al., 2011).

The simulation results indicated that, for lower demands, the ACC results in slightly increased speeds, while in congestion, the ACC increases the speed significantly. Congestion is eliminated even for the lowest market penetration scenario tested (20\% ACC). One potential disadvantage of the ACC is that bottlenecks can be created at locations where a significant number of drivers are likely to turn their ACC off. The ACC is based on the concept of constant time headways, and it results in a decrease in throughput because the ACC produced, on the average, longer time headways. When LCA technology was present without ACC, the number of lane change maneuvers and throughput increased, but the travel time remained constant. When both LCA and ACC were present, conditions improved significantly (Elefteriadou et al., 2011).

\subsubsection{Connected Vehicles}

In the United States, the Connected Vehicle (CV) effort has been led, insignificant part, by the United States Department of Transportation (USDOT). It is envisioned that every car manufactured in the U.S. will be equipped with a communications device and a GPS unit. The goal is to provide a communications link between vehicles on the road (vehicle-to-vehicle communication - V2V) and an instrumented road system (vehicle-to-infrastructure communication - V2I) in order to increase the safety, efficiency, environmental sustainability, and convenience of the transportation system.

The Connected Vehicle concept is supported by the development and prototyping of a particular type of wireless communications technology, referred to as dedicated 
short-range communications (DSRC). DSRC is a main contender for a communications method due to its technological advantages, such as fast, secure, two-way and broadband connections in a mobile environment. It is the only technology that meets the requirements for the safety applications of CV (Willke et al., 2009). DSRC allows drivers within a certain distance of each other to be connected. That means two vehicles or a vehicle and infrastructure can exchange information only when their distance is less than a certain distance.

Although DSRC communication is required for safety application, cellular communication technology satisfies many mobility applications of Connected Vehicle. In all likelihood, combinations of Connected Vehicle with DSRC and cellular communication technology will be used in the future, in combination with the autonomous vehicle technology described in the previous section.

\subsubsection{V2V Applications}

Using Vehicle-to-Vehicle (V2V) communication, each vehicle will know where other vehicles are located, whether in blind spots, stopped ahead on the highway but hidden from view, around a blind corner, or blocked by other vehicles. In fact, V2V has the ability to replace all of the sensors and cameras with one advisory sensor that provides all-around, instantaneous traffic intelligence. This promises a better and significantly less costly way of sensing other vehicles in the vicinity while driving. The information received from the devices can be transmitted to the driver through visual, audible and tangible warnings. 
Park et al. (2011) evaluated the deployment of freeway merge assistance to provide advisory messages at heavy weaving areas through V2V using VISSIM simulation. The evaluated algorithm calculates the acceleration rates and headway based on the collected gap information on freeway lanes. If the headway is greater than a minimum safety distance, the advisory is produced. The results indicated that in addition to safety benefits, this assistance can improve the mobility as well; however, it requires at least a $90 \%$ compliance rate to work properly.

Rim et al. (2011) developed a travel time estimation model that uses V2V and V2I to estimate lane-level travel times. They modeled a 4.6-mile highway segment in the VISSIM simulation software. They found that with a $20 \%$ or higher market penetration, a mean absolute relative error in travel time estimation of $6 \%$ to $8 \%$ is achievable.

Ni et al. (2012) considered a more general scenario, which incorporates three types of driving modes enabled by Connected Vehicle technology: non-CV, CV assisted, and $\mathrm{CV}$ automated. In the $\mathrm{CV}$-assisted mode, drivers receive advisories and safety warnings, while in the CV-automated mode, a vehicle is operated by CV-enabled automatic driving features; however, the driver may take over at any time. The purpose of this study was to estimate the capacity benefits of CV technology using CORSIM simulation. The results indicated a $20 \%$ to $50 \%$ increase in capacity when CV is fully deployed.

\subsubsection{V2I Applications}

One type of application of Connected Vehicle is the safety application, which is designed to increase situational awareness and reduce or eliminate crashes. There are 
various types of safety applications; three main safety applications of Connected Vehicle are the suggestive messages advising drivers about potential dangers, urgent messages warning drivers to take immediate actions, and secondary actions taken by vehicular control when drivers fail to comply with warnings or advisories. For example, the cooperative Intersection Collision Avoidance System (CICAS) is a major application that aims to improve intersection safety using these three applications (Elefteriadou et al., 2011). According to a study by Najm et al. (2010), these applications could potentially address about $75 \%$ of all crashes involving all vehicle types.

The Connected Vehicle technology promises to provide a data-rich travel environment. One important application of the V2I is probe vehicle data collection. The transportation communications network captures real-time data from on-board units located inside vehicles such as automobiles, trucks, and buses. The three major elements in V2I communication for collecting probe data are: On-Board Unit (OBU), Roadside Equipment (RSE), and a mobile communication technology such as DSRC. OBUs are installed in vehicles to record vehicle activity data during certain time intervals. These recorded activities are called "snapshots," which include data such as speed, position, turn signal activation, brake status, airbag activation and so on. OBU memory size is limited, and the total number of snapshots that can be stored in these devices is called the "buffer size.” When a vehicle enters a RSE coverage range, the information is transmitted to the infrastructure, and the memory of the OBU is cleared.

Periodic snapshots are recorded at set time intervals. Based on current protocol (SAE J2735), the recording time interval is set based on the vehicle speed. If the speed is greater than $60 \mathrm{mph}$, the recording time of travel is 20 -second intervals. For speeds less 
than $20 \mathrm{mph}$, the snapshots are recorded at 4-second intervals. For speeds between 20 $\mathrm{mph}$ and $60 \mathrm{mph}$, a linear interpolation is used to calculate the intervals. When a vehicle does not move for five seconds, periodic snapshots are no longer recorded. When the speed exceeds $10 \mathrm{mph}$, the snapshots are recorded again. Event-triggered snapshots are recorded when vehicle status elements change (such as airbag activation). However, Dion et al. (2010) recommended fixed-interval snapshots, preferably at short intervals. Also, they recommended recording snapshots while the vehicles are stopped.

Shladover and Kuhn (2008) investigated the quality of Connected Vehicle probe data for adaptive signal control, incident detection, and weather condition monitoring systems. Assuming 100\% market penetration, they concluded that the data collected based on current probe data protocol provides an acceptable representation of normal traffic conditions, assuming 1- to 2-minute data latency is acceptable.

Dion et al. (2011) evaluated the probe data generated in the Connected Vehicle environment using the PARAMICS traffic simulation software. They performed sensitivity analysis on the effect of the number of RSEs, RSEs communication range, OBU buffer size and snapshot generation interval, and market penetration on the utility of probe data. They also investigated the quality of link travel time estimates from Connected Vehicle probe data.

Kianfar et al. (2013) investigated a Genetic Algorithm-based optimization method to find optimal placements of RSEs in the urban network for the purpose of travel time estimation using the VISSIM traffic simulation software. The results suggested that in order to improve accuracy for a limited number of RSEs, the travel time estimation interval should be increased. 
Li et al. (2012) developed an event-based method that uses probe data and signal timing to estimate the queue length. Different from data collected using loop detectors, probe data can provide a lower bound on the queue length, even if the market penetration rate is low. The result showed when the penetration rate is $50 \%$, the mean absolute percentage error (MAPE) is less than 18\%; and, for low penetration such as $10 \%$, MAPE is around $60 \%$.

Because traffic flow information is not a reliable indicator of congestion, Kattan and Saidi (2012) developed a probe-based adaptive ramp metering based on CV data and compared the results with a detector-based and pre-timed ramp metering approach using PARAMICS micro-simulation. The probe-based approach takes as its main input the space mean speed extracted from vehicle probes moving constantly on the entire freeway. The results indicated that the probe-based algorithm outperformed the two other algorithms. The sensitivity analysis showed that larger penetration rates would not significantly change the results. A $10 \%$ penetration rate is expected to be enough for a reliable probe-based ramp metering. The results showed that the probe-based ramp metering still performs better than other algorithms at low penetration rates like 3\%. However, for very low penetration, such as $1 \%$, the detector-based algorithm produces better results.

Instead of relying on limited data from point detectors, Goodall et al. (2012) developed a rolling horizon traffic signal control algorithm called the "predictive microscopic simulation algorithm (PMSA)" to minimize delay over a 15-second period using data from V2I connections. Simulation results showed that with greater than a 50\% penetration rate at low- and mid-level volumes, the algorithm is able to significantly 
improve the performance. However, the performance worsens during saturated and oversaturated conditions.

Recently, CV data was used in queue length estimation. In the literature, researchers used this estimation to increase the effectiveness of adaptive traffic signal controllers and avoid queue spillback upstream intersections. Christofa et al. (2013) developed two different queue spillback detection methods based on Connected Vehicle data. The first one, called "gap-based detection," is based on the stopping position of the last equipped vehicle that joins the queue. The second method, the shockwave-based detection, is based on Connected Vehicle data and signal timings of the upstream intersection. The results showed that for different penetration rates, both methods can detect the occurrence of spillbacks in more than $80 \%$ of the cycles. Venkatanarayana et al. (2011) used the position of the last Connected Vehicle in the queue to find the queue length. Comert and Cetin (2009) presented a method based on distribution of the number of queued vehicle and market penetration rate and the position of the last Connected Vehicle to find the number of vehicles in the queue. Even if one CV is queued, their methodology is able to estimate the queue length.

Connected Vehicle technology was also recommended for transit priority. With real-time data about passenger loadings and current schedule adherence, the priority can be set more intelligently, which makes public transportation more efficient (Zeng et al., 2012). Liao et al. (2007) conducted a simulation study to take advantage of the already equipped GPS/automatic vehicle location system on the buses. The results indicated up to a 15\% decrease in bus travel time during peak hours. 


\subsection{Summary}

In this chapter, a comprehensive review was described first on topics related to traffic breakdown, and micro-simulation calibration. Since traffic simulation models are widely used and increasingly applied for the assessment of transportation systems, it is important to have a well-calibrated model in order to obtain a reliable assessment.

Later in this chapter, VSL systems implemented in the real-world are reviewed and their effects on traffic safety and mobility are discussed. Active Traffic and Demand Management (ATDM) strategies such as variable speed limit (VSL) are state-of-the-art methods that are increasingly being considered to improve the efficiency of the existing freeway system. VSL strategies identify and disseminate the appropriate speed limit based on the prevailing traffic conditions, road surface conditions, and weather conditions. Although the traffic safety benefits of implementing the VSL system in the real-world are well-established, very few of the previously developed VSL strategies have documented improvement on traffic mobility. Finally, a review of different applications of Connected Vehicle was conducted. 


\section{CHAPTER 3}

\section{METHODOLOGY}

This research aims to develop a VSL strategy in order to mitigate freeway congestion caused by traffic breakdown at bottlenecks. This chapter presents the methodology used in achieving the objectives of this study.

\subsection{Simulation Calibration}

As stated earlier, microscopic simulation has been used to assess advanced strategies, such as ramp metering and VSL, to reduce the probability of breakdown. Well-calibrated simulation models are critical to achieving the specific objectives of the advanced strategies' assessment process; the calibration process must consider these objectives. As mentioned in the previous section, researchers developed methodologies and guidelines for traffic simulation model calibration. One of the most important and widely used guidelines in this regard is the Federal Highway Administration's guideline presented in the Traffic Analysis Toolbox Volume III and IV (Dowling et al., 2004; Holm et al., 2007). According to FHWA guidelines, the calibration methodology consists of three steps: 1) capacity calibration, 2) traffic volume calibration, and 3) system performance calibration. The calibration procedure developed in this study extends the FHWA procedure by including an additional step that involves the consideration of breakdown characteristics, which is critical for successful assessments of advanced traffic management strategies that address breakdown when using simulation models for this purpose. Figure 3-1 shows an overview of the modified calibration 
procedure presented in this research. In this figure, the additional step, which shows the breakdown characteristics calibration process, is highlighted by the dotted line.

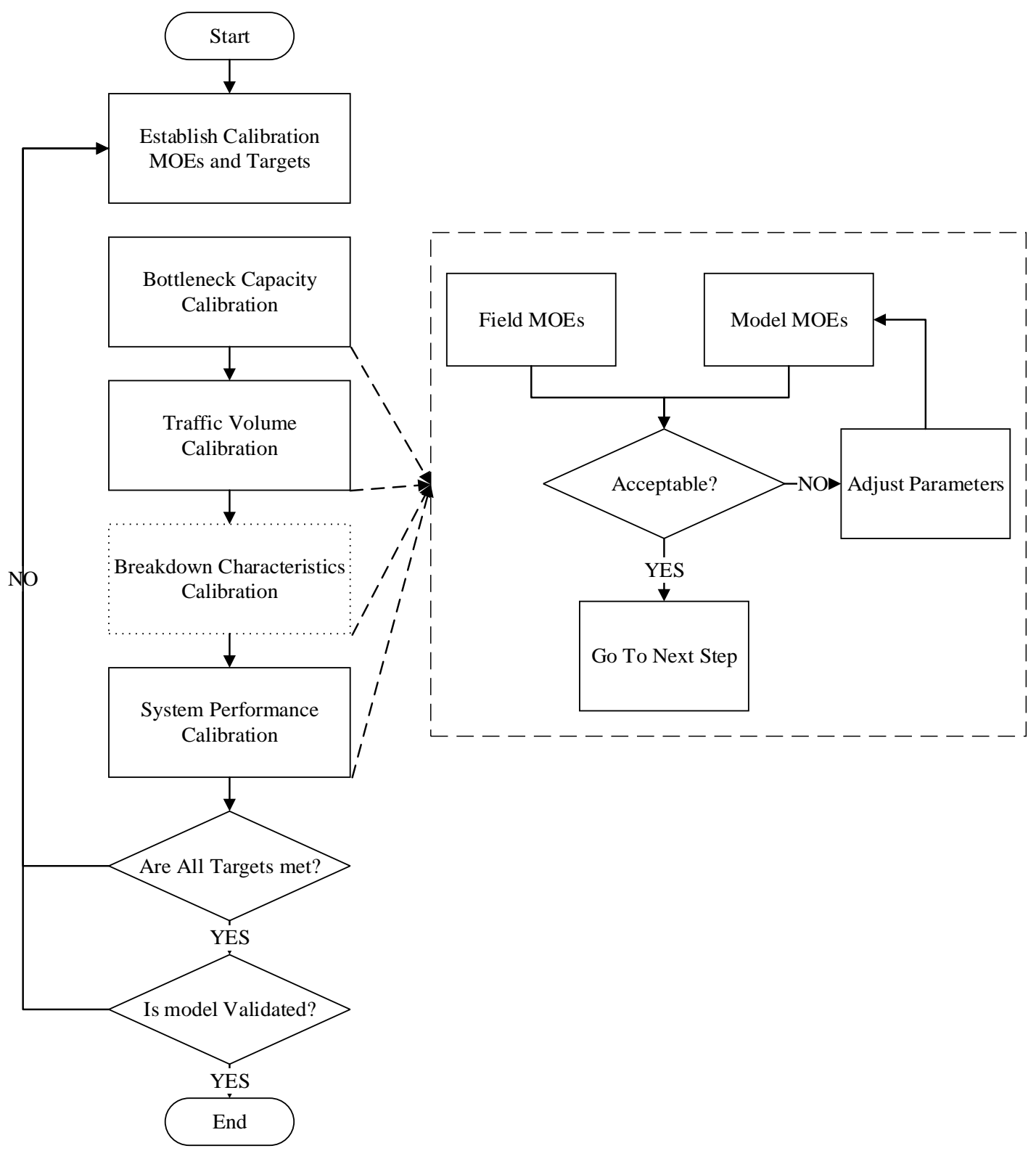

Figure 3-1: Calibration procedure

In this research, the procedure is tested using the CORSIM micro-simulation tool (Version 6.2). CORSIM is a stochastic micro-simulation model that was developed based on the FHWA's developments of simulation models in the 1990s, and consists of two 
traffic simulation models: (a) NETSIM for simulating arterials, and (b) FRESIM for simulating freeways. The CORSIM micro-simulation model has three main sets of calibration parameters related to driver behavior on freeways: free-flow speed, car-following, and lane-changing parameters. The free-flow speed parameters in CORSIM consist of the mean free-flow speed and the free-flow speed multipliers. The mean free-flow speed is a link-specific parameter. Using the HCM procedure, the free-flow speed can be estimated as follows:

$$
F F S=75.4-f_{L W}-f_{L C}-3.22 T R D^{0.84}
$$

where

$$
\begin{aligned}
F F S & =\text { estimated free-flow speed (mph), } \\
f_{L W} & =\text { adjustment factor for lane width }(\mathrm{mph}), \\
f_{L C} & =\text { adjustment factor for lane width (mph), and } \\
T R D & =\text { total ramp density (ramp/mile). }
\end{aligned}
$$

The free-flow speed can also be estimated based on field estimation. The free-flow speed multiplier is a global parameter, and it is a percentage multiplier for each driver type of the mean free-flow speed. A more aggressive driver receives a higher multiplier, reflecting a higher free-flow speed. The multiplier specification provides a distribution of free-flow speed by driver type.

CORSIM uses the PITT car-following model, which incorporates the vehicle spacing and speed differential between the lead and following vehicle as two independent variables, as follows: 


$$
d_{F L}=L_{L}+P I T T+k u_{F}+b k\left(u_{F}-u_{L}\right)^{2}
$$

where

$$
\begin{aligned}
& d_{F L}= \text { vehicle spacing between the front bumper of the lead vehicle and the front } \\
& \text { bumper of the following vehicle, } \\
& L_{L}= \text { the lead vehicle length, } \\
& \text { PITT }=\text { car following constant of PITT car following model, } \\
& u_{F}=\text { speed of the following vehicle, } \\
& u_{L}=\text { speed of the lead vehicle, } \\
& k=\text { driver sensitivity constant, and } \\
& b=\text { calibration constant which equals } 0.1 \text { if the speed of the following vehicle } \\
& \quad \text { exceeds the speed of the lead vehicle; otherwise, it is set to zero. }
\end{aligned}
$$

Basically, the rule is that the following vehicle maintains a safe distance gap from the lead vehicle, and in the case where the gap is not sufficient, the follower vehicle decreases the speed. The default car-following sensitivity factors for CORSIM, which reflect the aggressiveness of the drivers and govern how vehicles follow one another, are represented by a discrete distribution that starts with a value of 1.25 , which decreases at an increment of 0.10 by driver type, to a value of 0.35 for the most aggressive drivers. The calibration of the driver sensitivity factor can be achieved by changing the driver distribution, which is a global parameter and/or changing link-specific adjustment parameter.

Lane-changing can be categorized in three groups: 1) mandatory due to blocked lane, exiting freeway or lane drop; 2) discretionary is based on whether the driver is satisfied with driving conditions in his/her current lane, and 3) anticipatory because of 
warning signs. The lane-changing algorithm inputs are affected by the characteristics of drivers, vehicle performance, and prevailing traffic conditions. In CORSIM, the lane-changing algorithm can be calibrated using a number of parameters, including the time-to-complete-a-lane-change, the gap acceptance parameter, the percent driver yielding at merge point, the multiplier that simulates the desire for a discretionary lane change, and the advantage threshold for a discretionary lane change. As mentioned above, there are a large number of parameters to be considered when calibrating a microscopic model like CORSIM, which translates into a number of combinations of these parameter values. In addition, many of the parameters are continuous variables rather than discrete, increasing the number of possible solutions. The complexity of the calibration can be illustrated by considering the following example. If 10 parameters need to be modified in the calibration and each discrete parameter has 5 levels, then there are $5^{10}=9,765,625$ combinations that need to be tested. As reported in the reviewed literature, many of proposed heuristic optimization-based calibration approaches require long simulation running times and may produce parameters that are not notably different than manually calibrated parameter sets. Furthermore, in some cases, these methods fail to consider important aspects of the modeling process that can be captured by experienced modelers using manual calibration. Optimization methods can still reduce the effort required for calibration and the dependency on the expertise and judgment of the users. A manual trial-and-error calibration approach is used in this study. However, the concepts discussed hereinafter can be extended to automated calibration methods in future works. 


\subsubsection{Capacity Calibration}

Figure 3-2 shows an overview of the capacity calibration procedure; identifying the locations of bottlenecks is the first step in capacity calibration in simulation modeling. There are several methods for identifying bottlenecks. With the increased deployments of traffic detectors associated with traffic management systems, the simplest way to identify bottleneck location is by using traffic detector-measured speed. The approximate bottleneck location is between the most downstream detector with congestion and its downstream detector location at a free-flow condition.

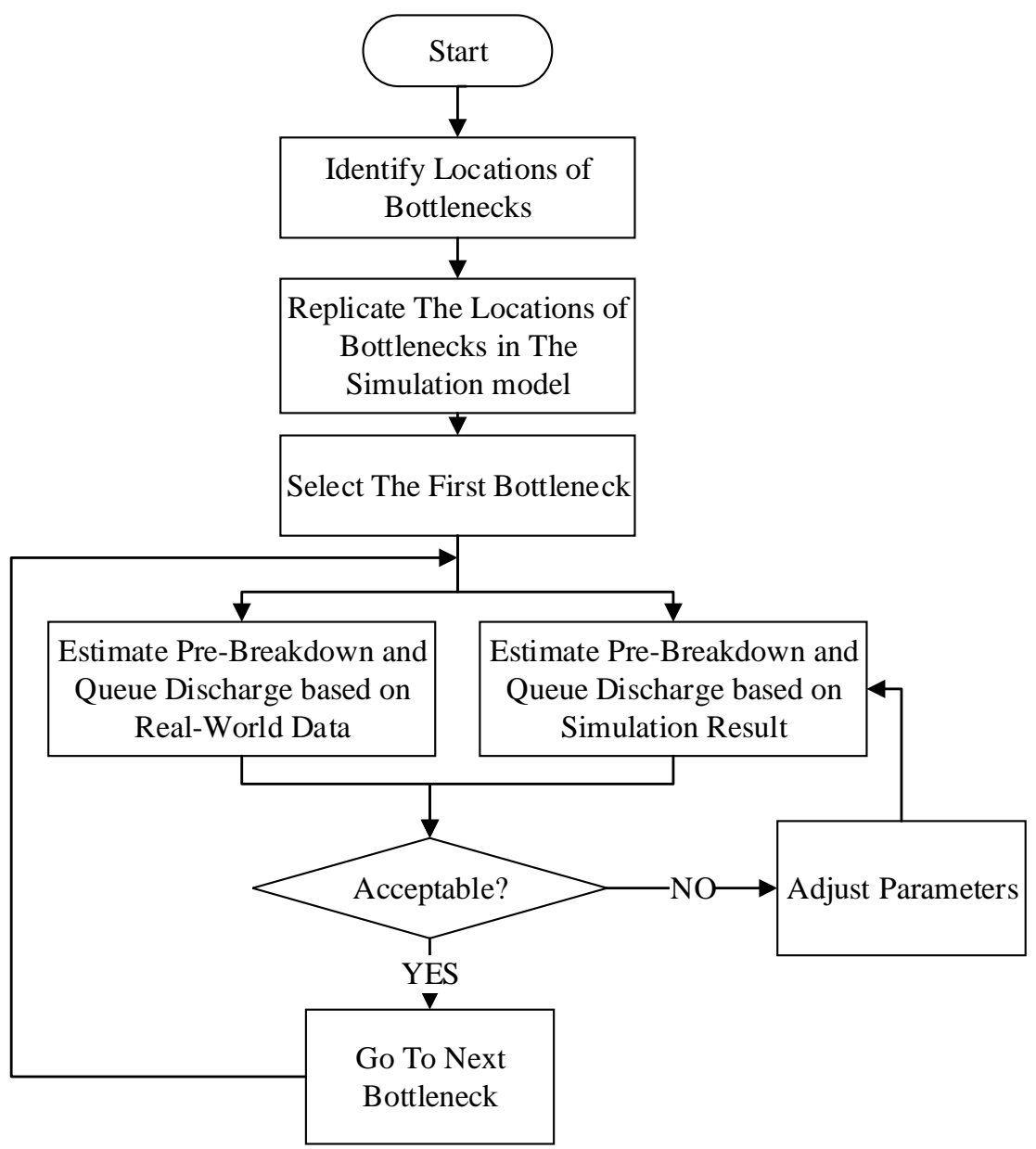

Figure 3-2: Capacity calibration procedure 
According to the FHWA simulation guidelines (Dowling et al., 2004), a visual audit can be used as the primary method for identifying bottleneck locations. Utilizing contour maps is a widely used method to visualize traffic conditions. Speed, volume, or occupancy can be visualized on a time-space diagram utilizing a color scheme representing the range of traffic conditions. These maps can be used as an effective tool for bottleneck analysis, since they provide a clear image of existing traffic conditions, including the locations of the congested areas and the extent of congestion. However, it is important to consider multiple days in the analysis to ensure that a bottleneck is a recurring bottleneck and that the model is not over-fitted to one day. If incident and weather data are available, then the days with incidents and bad weather conditions should be isolated in the analysis.

The HCM procedure allows for calculating capacity based on the geometric conditions of the facility and treats it as a deterministic value. However, it is preferable to measure capacity based on the maximum flow before breakdown and the queue discharge rate during congestion in the field to account for site specifications. The queue discharge rate is defined as the long-run average of flow during the breakdown period. The maximum pre-breakdown flow was measured at different aggregation intervals, such as one minute, five minutes or fifteen minutes immediately before the breakdown occurs. Figure 3-3 shows how the pre-breakdown flow and queue discharge rates are identified in this study, based on the above discussion. The FHWA guidelines recommend the use of the queue discharge rate as the capacity in simulation model calibration, since it is more stable and easier to measure. For modeling and assessing the benefits of advanced strategies that seek to prevent or delay breakdown, it is important to examine both the 
maximum pre-breakdown flow and queue discharge rate. Thus, in this research, both parameters are considered in the calibration. This is important since estimating accurate capacity drops, which is the difference between the maximum pre-breakdown flow and the queue discharge rate, is necessary to evaluate the advanced alternative strategies that are proposed to reduce congestion. It is not clear whether the calibrated simulation models are able to replicate the drop in throughput due to the difference between pre-breakdown capacity and queue discharge rate. This study, in addition to pre-breakdown flow and queue discharge, considers the fundamental diagram for further capacity analysis. Fundamental diagrams are constructed from both simulation runs and real-world data to present the flow-occupancy relationship at the bottlenecks. This fundamental diagram is important since it shows the variation of capacity between days; it can also show the critical density where flow reaches capacity, among other things, which are important to modeling breakdown.

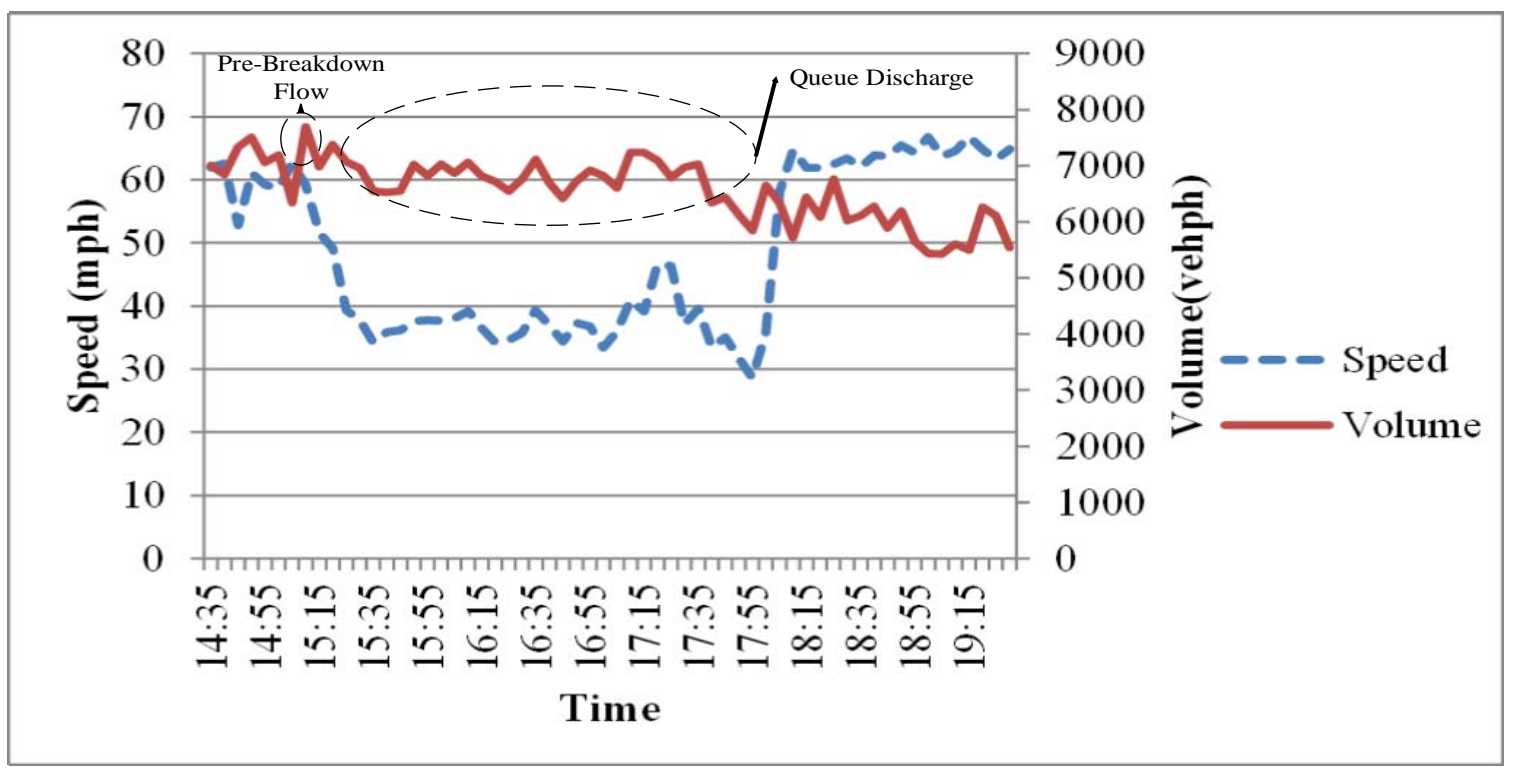

Figure 3-3: Pre-breakdown flow and queue discharge rate estimation 
It is important that the micro-simulation model replicates the bottleneck locations and the capacities at these locations. At this stage, network-wide parameters, such as the car-following sensitivity factors and lag acceleration and deceleration time are fine-tuned for the purpose of capacity calibration. Later, as there may be multiple bottlenecks on the facility with different characteristics, the car-following sensitivity multiplier, which is a link-specific parameter, may need to be fine-tuned for each bottleneck individually. The link-specific parameter calibration does not mean only adjusting the link parameter for the bottleneck link, as it may need to adjust the parameter of upstream links, as well as to represent local conditions properly. In addition, this adjustment should be made to obtain measured or estimated capacity and queue discharge rate and not merely to produce the observed queue lengths.

\subsubsection{Traffic Volume Calibration}

Volume-based calibration will result in a model with traffic volumes that are close enough to observed traffic volumes. In this study, a node diagram is used as an important tool to have a better understanding of the volume variation in space for each time interval (15 minutes in our case) compared to field data. The goal of the calibration process is to reach a good fit to real-world volume. Figure 3-4 shows an overview of the traffic volume calibration procedure.

Given that all input volumes are correct and verified and the bottleneck capacities are calibrated, this step is necessary to ensure correct parameters to eliminate any artificial bottlenecks in the simulations. This is achieved by changing calibration parameters, especially link-specific parameters such as the reaction points for the cars 
exiting at the off-ramps, which was found to have significant influence in creating artificial bottlenecks if not well set. Since this data is not available from field measurements, the right values are found using the trial-and-error approach to prevent the generation of unrealistic bottlenecks.

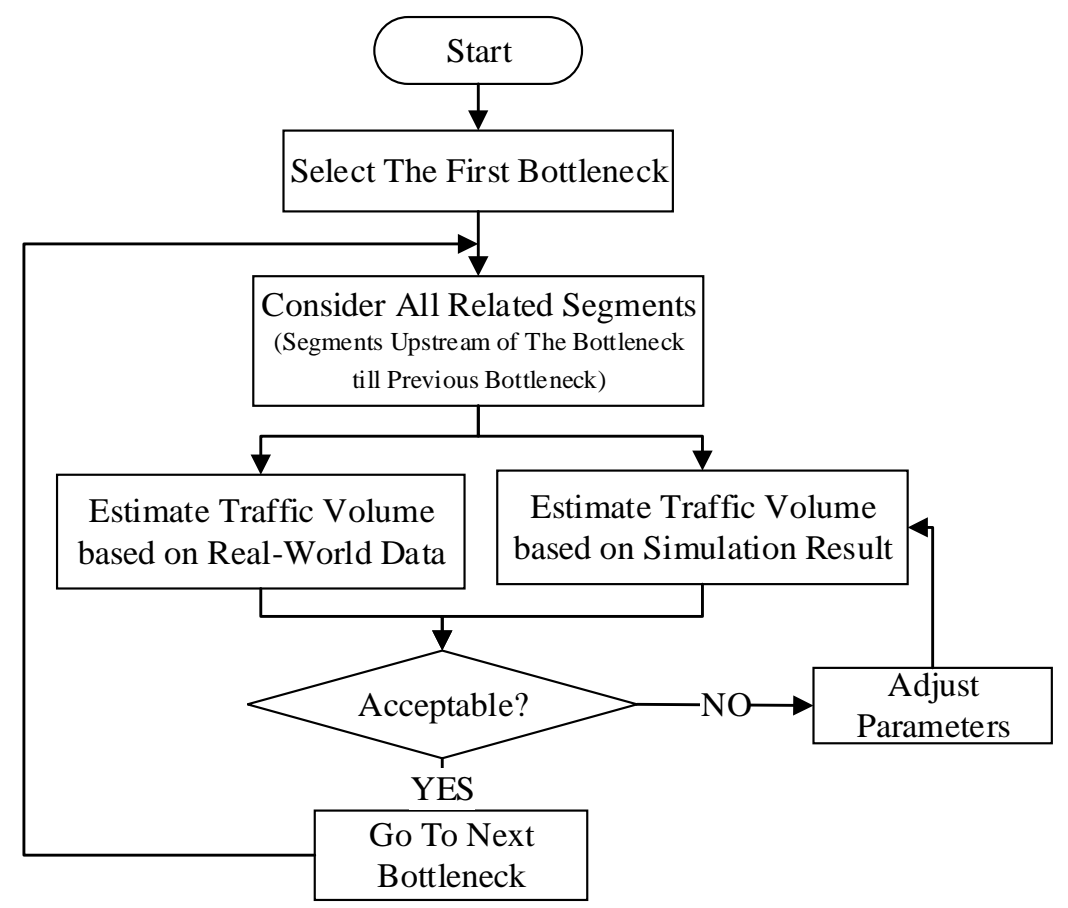

Figure 3-4: Traffic volume calibration procedure

As defined by FHWA guidelines, the MOE criteria used in volume calibration is defined based on Geoffrey E. Havers (GEH) statistics, as follows:

$$
G E H=\sqrt{\frac{2(E-V)^{2}}{E+V}}
$$

where $E$ is simulation estimated volume and $V$ is a field volume. GEH is computed at each time interval (15 minutes) for each individual link. At each time interval, GEH should be less than 5 for at least $85 \%$ of freeway links. In addition, it is recommended to 
compute the GEH for the whole network by summation. The resulting network-wide GEH should be less than 4 .

In order to come up with a well-calibrated model, the volume-related MOE statistics are estimated individually for each bottleneck location. For the most upstream bottleneck, the MOE statistics are calculated for the links upstream of that bottleneck and link-specific parameters are adjusted. Once the simulated output meets the criteria at this bottleneck location, the calibration procedure moves to the next bottleneck. In other words, in each step, the focus is on calibrating the links related to one bottleneck, between the upstream bottleneck and this bottleneck to reach desirable results. This procedure continues until the entire network satisfies the calibration criteria. If all links have not reached desirable and required accuracy, the procedure starts again.

\subsubsection{Breakdown Characteristics Calibration}

When modeling advanced strategies, the breakdown characteristics at the bottleneck, in addition to capacity and queue discharge rates, must be examined. Aside from the main causes of breakdown, traffic breakdown has other characteristics that need to be examined, as mentioned in the literature review, such as the average speed during breakdown, duration of breakdown and the beginning and end time of breakdown. Figure 3-5 shows an overview of the breakdown characteristics calibration procedure.

In addition to the abovementioned characteristics, the breakdown stochasticity is another important consideration. It was observed that, in the real-world, at the same bottleneck location and for the same combinations of ramp and freeway flows, breakdown may or may not occur. When it occurs, it can occur at different times. This 
stochastic nature of capacity has received a great amount of interest and attention among researchers in two areas: 1) its impacts on a freeway's capacity assumptions (Geistefeldt, 2008; Elefteriadou et al., 2003), and 2) finding the probability of breakdown occurrence based on upstream traffic conditions such as traffic flow, occupancy or combinations of the variables (Elefteriadou et al., 1995, Kondyli, 2011).

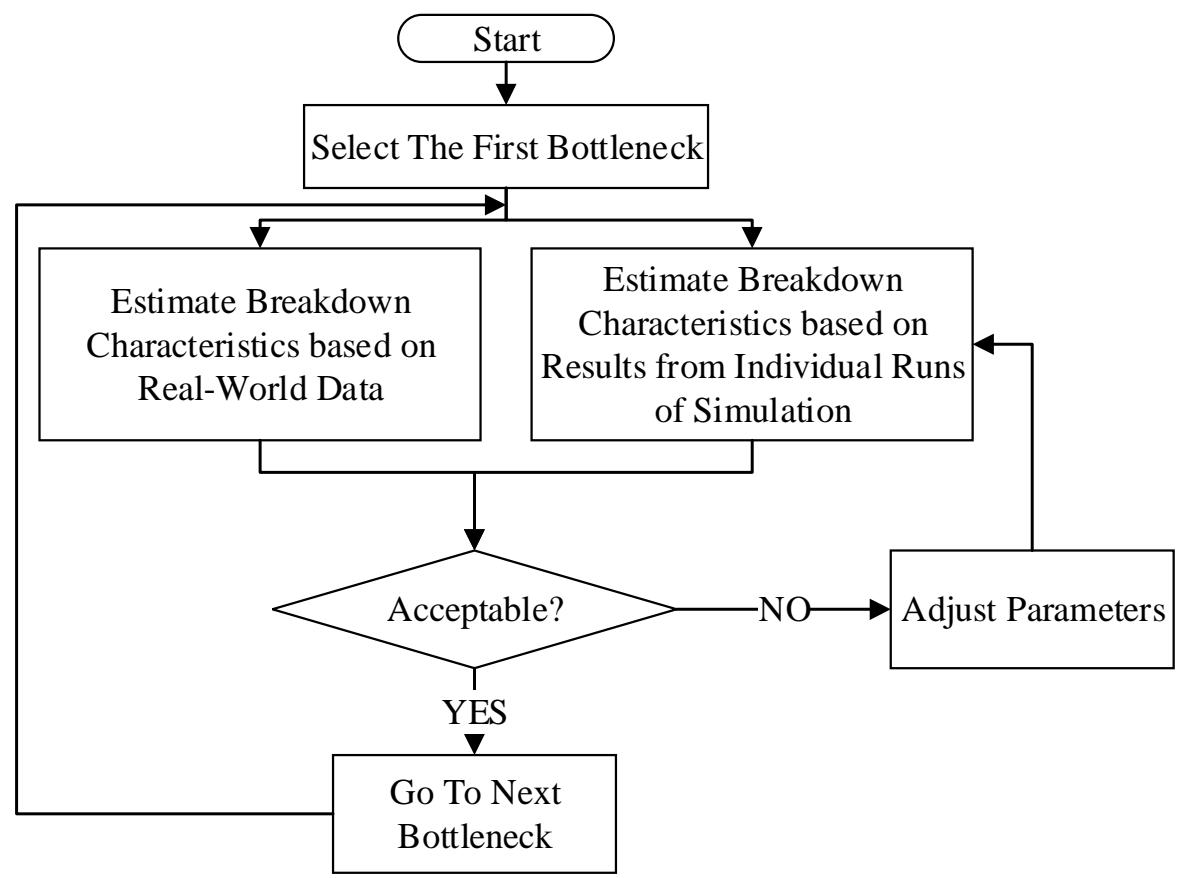

Figure 3-5: Breakdown characteristics calibration procedureThe goal of this research is to provide a procedure to extend the calibration of simulation models and also consider breakdown characteristics. This calibration, combined with the capacity and queue discharge calibration, is meant to consider the operations at the bottleneck location in a greater level of details, as needed when modeling strategies prevent breakdown. As mentioned, there are different definitions of breakdown occurrence (section 3.1.1). Estimates of the breakdown characteristics and bottleneck capacity depend on these definitions. In addition, traffic data contains noise, which reduces the accuracy of the 
estimated breakdown characteristics, such as the starting time of breakdown and its duration. Wavelet transform is an effective time-frequency decomposition tool that is widely used in analyzing and extracting information from non-stationary signal time-series.

This research uses the definition presented by Elefteriadou et al. (2011) and the wavelet transform method (section 3.5.) to identify the start and end times of breakdown occurrence, and the rest of estimates are based on this time window. After estimating the breakdown characteristics based on field measurements and simulation results, the link-specific parameters at the bottleneck locations are fine-tuned as necessary to simulate real-world conditions.

It is interesting to compare the real-world stochasticity mentioned above with the stochasticity of simulation models, described next. Stochastic simulation models produce different results for simulation runs with the same inputs, but different seed numbers. Thus, there is a need to run microscopic simulation models like CORSIM multiple times with different random seed numbers to account for the stochasticity of these models. The required minimum number of runs is determined based on the variance and the mean of the measures of effectiveness and acceptable confidence level, as follows:

$$
n=\left(\frac{s . Z}{\varepsilon}\right)^{2}
$$

where $\mathrm{n}$ is the minimum required number of model runs; $\mathrm{s}$ is the standard deviation of the examined performance measures; $\varepsilon$ is the required accuracy in the same units as performance measurement; and $\mathrm{Z}$ is the statistic value for a required confidence level. 
Testing the adequacy of the sample size in volume-based calibration and system performance calibration based on Equation (3-4) is necessary. However, the stochastic nature of breakdown and capacity requires additional consideration of how bottleneck capacity and breakdown characteristics calibration vary in the simulation runs, compared to real-world variations. The FHWA guideline reported that the results from individual runs for the same demands but different seed numbers in CORSIM micro-simulation can vary by up to 25 percent. Higher standard deviations may be expected for facilities operating at or near capacity. The above discussion leads to two reasons that individual model runs, each with different seed numbers, should be considered individually when examining breakdown characteristics and capacity calibration rather than averaging the results from all runs, as is currently done:

1) If the real-world breakdown stochastic nature is to be replicated in simulation, in one model run the breakdown may occur at a specific time, and in another run it may occur in another time, or it may even not occur at all. As Figure 3-6 shows, using the average value of system performance results in smoothed values that dilute the high congestion levels in runs with longer traffic breakdown durations. In addition, when examining the changes in average performances such as speed from multiple runs with time, sharp changes in these measures indicating breakdown are also eliminated.

2) A question is raised here: Can these variations between runs reflect real-world variations due to the stochastic variations in traffic stream characteristics? In other words, is a simulation model capable of assessing breakdown probability? This question has not been answered in the literature. 


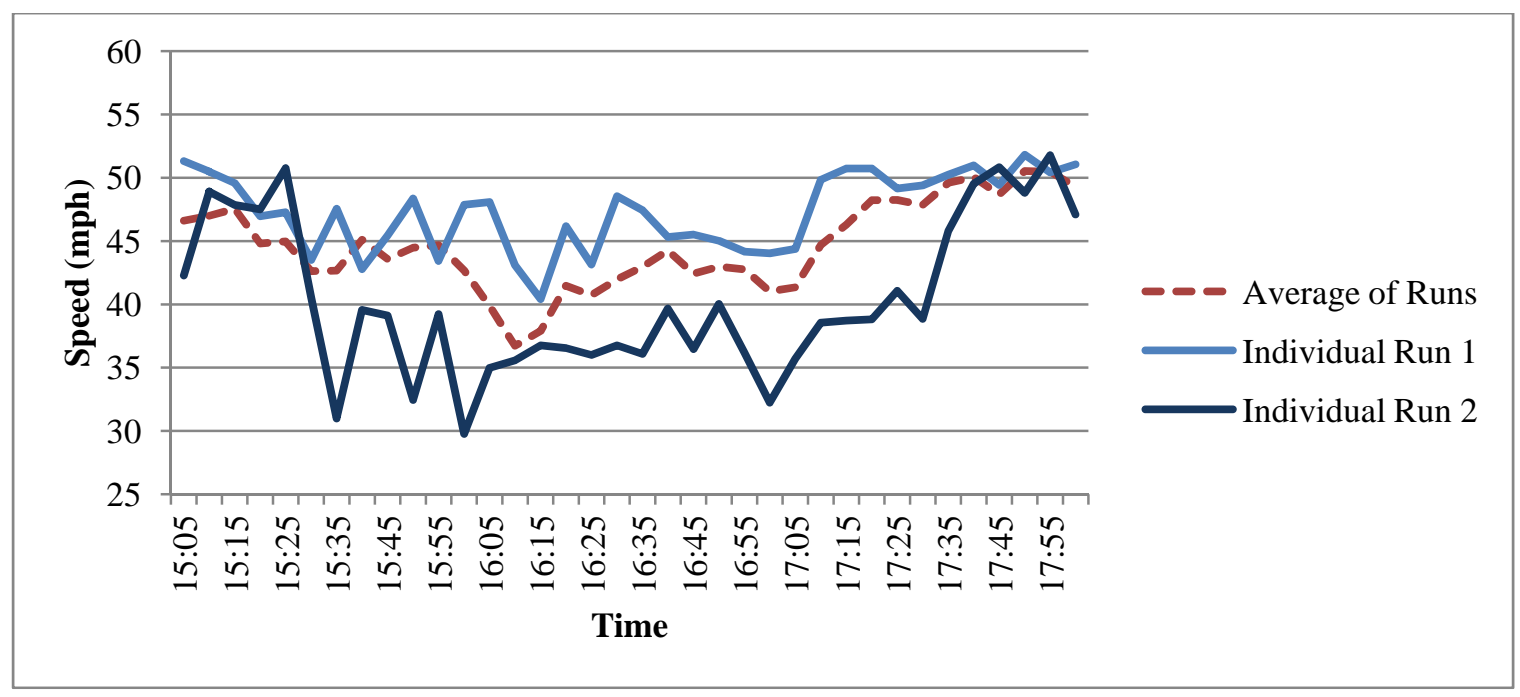

Figure 3-6: Speed data from CORSIM output for one individual run and average of runs

This study considers the probability of breakdown as one of its main characteristics. As a result, in this step of calibration, the probability of breakdown based on the simulation model is compared with the probability of breakdown based on real-world data. For these reasons, individual runs will have to be considered when studying simulation abilities to assess breakdown characteristics and capacity.

The probability of breakdown is modeled using a large sample size of traffic data, such as volume or occupancy, at the bottleneck location and upstream location. The probability of breakdown can be developed using the Kaplan-Meier method, which is a product-limit method (Kondyli, 2009). This estimation is non-parametric. The distribution function of the breakdown occupancy, $F(o)$, is:

$$
F(o)=1-\prod_{i: o_{i} \leq o} \frac{n_{i}-1}{n_{i}}
$$

where $o$ is the freeway occupancy, $o_{i}$ is the freeway occupancy during the breakdown interval $i$, and $n_{i}$ is the number of intervals with a freeway occupancy of $o$. This 
probability is calculated in this study based on real-world multiple day detector data and simulation runs with different seed numbers, and the results are compared.

\subsubsection{System Performance Calibration}

This is the last step of the four-step calibration process. By now, the simulation model is calibrated based on bottleneck capacity, traffic volume, and breakdown characteristics. This step ensures that system performance measures such as speeds, queue lengths and congestion levels are similar to field data. Additional fine-tuning of simulation model parameters may be required at this stage. The FHWA guideline points out that the visual audit of speed profile should be used as an important tool for this purpose. Comparing speed contour maps of simulation results against field detector data will show the ability of the calibrated model to adequately replicate speed patterns at bottleneck locations, queue build-up and dissipation, and the extent of congestion.

In addition to the visual comparison, this study uses the correlation coefficient (r) as a goodness-of-fitness between simulation and real-world values, as indicated in the following equation:

$$
r=\frac{1}{N-1} \sum_{i=1}^{N} \frac{\left(x_{i}-\bar{x}\right)\left(y_{i}-\bar{y}\right)}{\sigma_{x} \sigma_{y}}
$$

where $N$ is a number of estimates, $x_{i}$ is the simulated volume estimate at time step $i, y_{i}$ is the real-world volume estimate at time step $i, \bar{x}$ and $\bar{y}$ are sample average, and $\sigma_{x}$ and $\sigma_{y}$ are the sample standard deviation.

In addition, the simulation model is further calibrated using goodness-of-fit measures, including the mean absolute error (MAE), mean absolute normalized error (MANE), 
Theil's Inequality Coefficient, and root mean squared normalized percent error, (RMSNPE) as follows:

$$
\begin{gathered}
M A E=\frac{1}{N} \sum_{i=1}^{N}\left|x_{i}-y_{i}\right| \\
M A N E=\frac{1}{N} \sum_{i=1}^{N} \frac{\left|x_{i}-y_{i}\right|}{y_{i}} \\
\text { Theil's inequality coefficient }=\frac{\sqrt{\frac{1}{N} \sum_{i=1}^{N}\left(y_{i}-x_{i}\right)^{2}}}{\sqrt{\frac{1}{N} \sum_{i=1}^{N} y_{i}^{2}}+\sqrt{\frac{1}{N} \sum_{i=1}^{N} x_{i}^{2}}} \\
\text { RMSNPE }=100 \times \sqrt{\frac{1}{N} \sum_{i=1}^{N}\left(\frac{x_{i}-y_{i}}{y_{i}}\right)^{2}}
\end{gathered}
$$

where $N$ is a number of estimates, $x_{i}$ is the simulated estimate at time step $i, y_{i}$ is the real-world estimate at time step $i$. These measures are estimated for both speed and volume estimates.

MAE uses the absolute value of the difference between the observed and simulated measurements; thus, it gives equal weights to all errors, and consequently the measurements with larger errors will contribute more to the value of MAE. Some other measures (such as MANE, RMSNPE) depend on the normalized differences, which are percentage errors decided by the actual value of measurements. Even for a small difference, the small value of a measurement generates a larger percentage error, which might lead to overemphasizing on a minor fluctuation that is common in the nature of traffic phenomena. Simultaneous consideration of these two types of measurements in the analysis will help avoid these common mistakes. Furthermore, Theil's Inequality 
Coefficient is used to analyze how well the simulation model is able to replicate the variability in the field data.

\subsection{Background of the Developed VSL Methodology}

This research focuses on developing VSL strategies that address congestion caused by recurrent bottlenecks. Figure 3-7 shows how congestion starts at the bottleneck, as breakdown occurs, and how it propagates upstream of the bottleneck. As time passes, the traffic demand decreases and the bottleneck capacity (supply) surpasses demand, resulting in the dissipation of congestion.

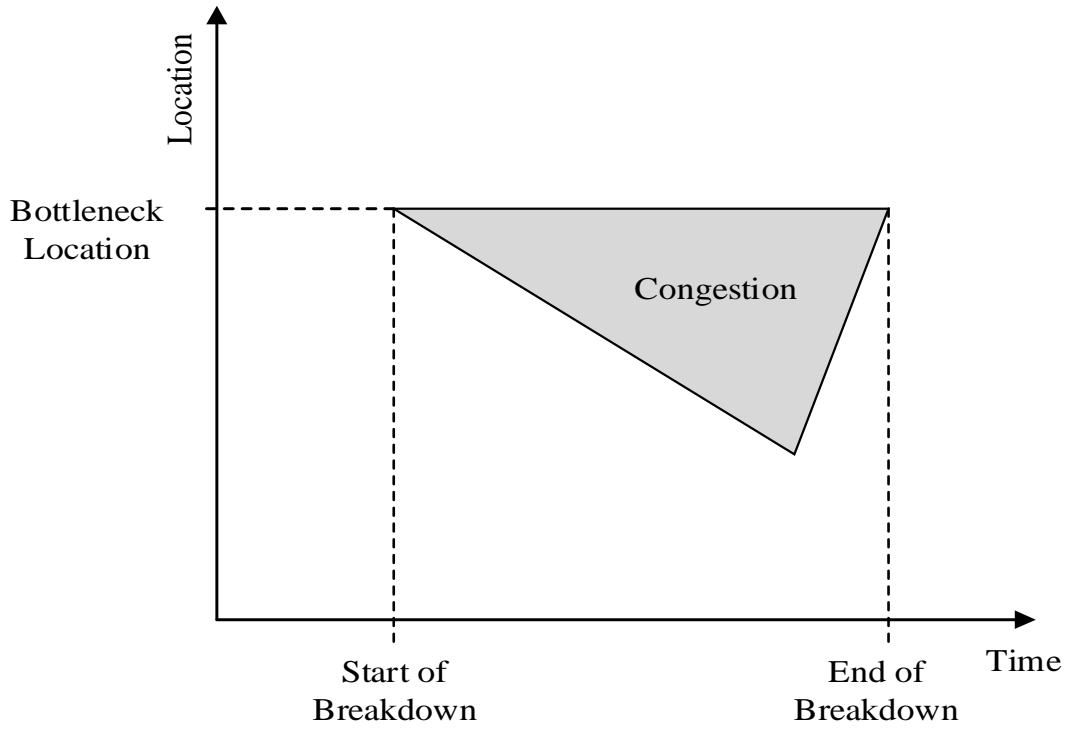

Figure 3-7: Traffic congestion build up and dissipation

During the development and testing of VSL strategies in this study, results showed that the mobility benefits from VSL can arise from two different mechanisms for the under-saturated and over-saturated regimes. This section presents an overview of the VSL operations during these two different traffic regimes.

Before breakdown occurs, the benefit of VSL eliminates or delays traffic breakdown. VSL reduces the average speed and increases travel time as the posted speed 
limit decreases. The amount of speed reduction depends on the driver compliance rate and the posted dynamic speed limit. At this stage, with VSL, the same traffic flow moves at lower speeds and higher occupancies than the non-VSL state, as shown in Figure 3-8. Although travel time increases due to lower speeds, the VSL can decrease travel time by avoiding or postponing breakdown occurrence.

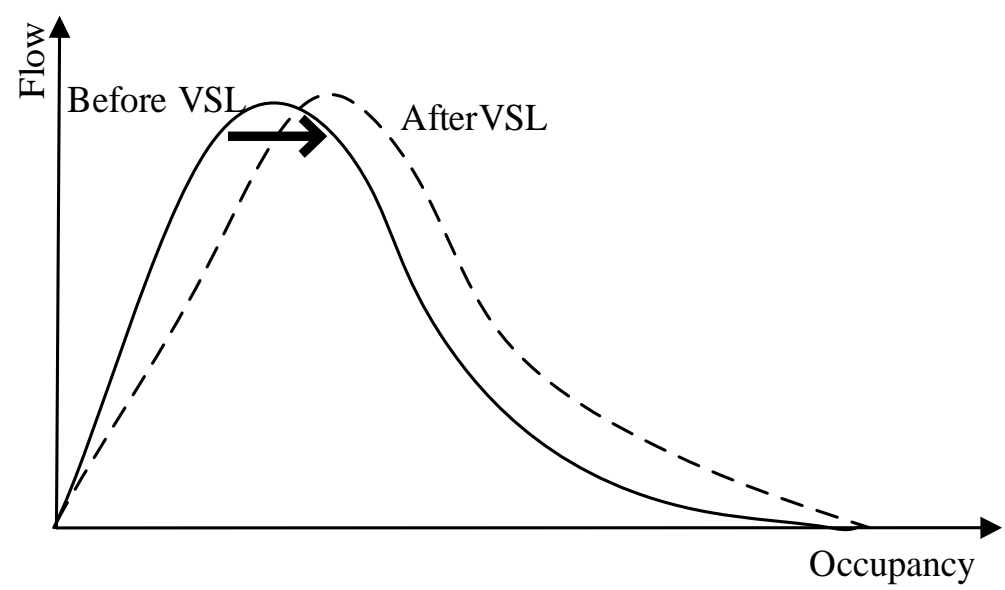

Figure 3-8: VSL impact on fundamental traffic diagram

Freeway capacity has a stochastic nature, which may cause different traffic patterns for the same demand levels. Field study results indicate two days with very similar traffic demands, but significantly different congestion levels and traffic conditions. This shows that breakdown occurrence has a stochastic nature as well. Analysis of field data has shown that breakdown could occur at different traffic flow levels. There has been a considerable amount of research and effort to find the probability of breakdown based on different indices, such as downstream volume and occupancy or the combination of on-ramp and upstream mainline volumes (Kondyli, 2009). Three traffic flow states are considered: 
- Free-flow conditions: In this state, the traffic flow is low enough that the probability of breakdown occurrence is zero.

- Light congestion conditions: In this state, traffic flow is low enough such that small disturbances vanish without impacts on traffic, but it is high enough that large disturbances result in breakdown occurrence. In other words, there is a probability of breakdown.

- Heavy congestion conditions: In this state, traffic flow is high enough that breakdown could occur any time if it has not already occurred. In other words, the probability of breakdown is close to one.

At low congestion levels, with zero or very low probability of breakdown, and at heavy congestion conditions, with a breakdown probability close to 1 , the VSL system has no room to reduce the probability of breakdown. However, in lower congestion conditions with moderate breakdown probability, the VSL is expected to reduce the probability of breakdown by reducing traffic flow disturbances through the decreasing of the input flow to the bottleneck location and achieving a more homogeneous distribution of speed.

The fundamental diagram of traffic flow shows the relationship between traffic flow and traffic density, and helps to better understand the traffic system's behavior. Figure 3-6 shows that when the VSL is applied prior to breakdown, it reduces the slope of the speed-occupancy relationship. The lower the posted speed limit, the larger the reduction in this slope. In addition, the VSL shifts the critical occupancy to higher values in the diagram, which means that the unstable region of the fundamental diagram shifted to the right. This indicates that the unstable region will start at a higher density with the 
VSL. While this shift in the fundamental diagram may result in an increase in freeway throughput, if the posted VSL is set too low, it may decrease freeway capacity since the free-flow speed and capacity are related. This may result in traffic demands exceeding the capacity of the VSL influence area upstream of the bottleneck location. The above discussion indicates that there is a site-specific optimal speed limit to balance the different speed limit effects prior to breakdown.

After the occurrence of traffic breakdown, not only does the traffic speed decrease and congestion increases, but the freeway maximum possible throughput can drop as well. The average traffic speed in the congested area is significantly less than the posted speed limit, which means that lowering the speed limit in the congested segments has no effect on traffic performance. However, the VSL system activated ahead of the congested area can still influence the congestion after breakdown occurrence. During congestion, the VSL mobility benefits are estimated to be due to suppressing backward congestion shockwaves by reducing the inflow of traffic to the congested area. This is expected to result in reducing the growth of queues and thus the extents of traffic congestion. To achieve this goal, as the congested area propagates upstream, the VSL location should be pushed upstream as well.

It has also been suggested that at the head of the queue, specifically around the location of queue discharge, the vehicles should accelerate to increase throughputs (Carlson, 4). Deactivation of the VSL at the head of congestion, upstream of the bottleneck, could help vehicle acceleration. Carlson (2010) suggested that the deactivation of the VSL should be made 0.3 - 0.6 mile ahead of the bottleneck location. This will potentially increase capacity at the bottleneck since capacity is a function of the 
traffic flow speed. In addition, as HCM points out, the higher free-flow speed results in a higher capacity of freeway, as shown in Figure 3-9. Free-flow speeds are closely related to posted speed limits. Figure 3-10 shows an overview of the VSL strategy.

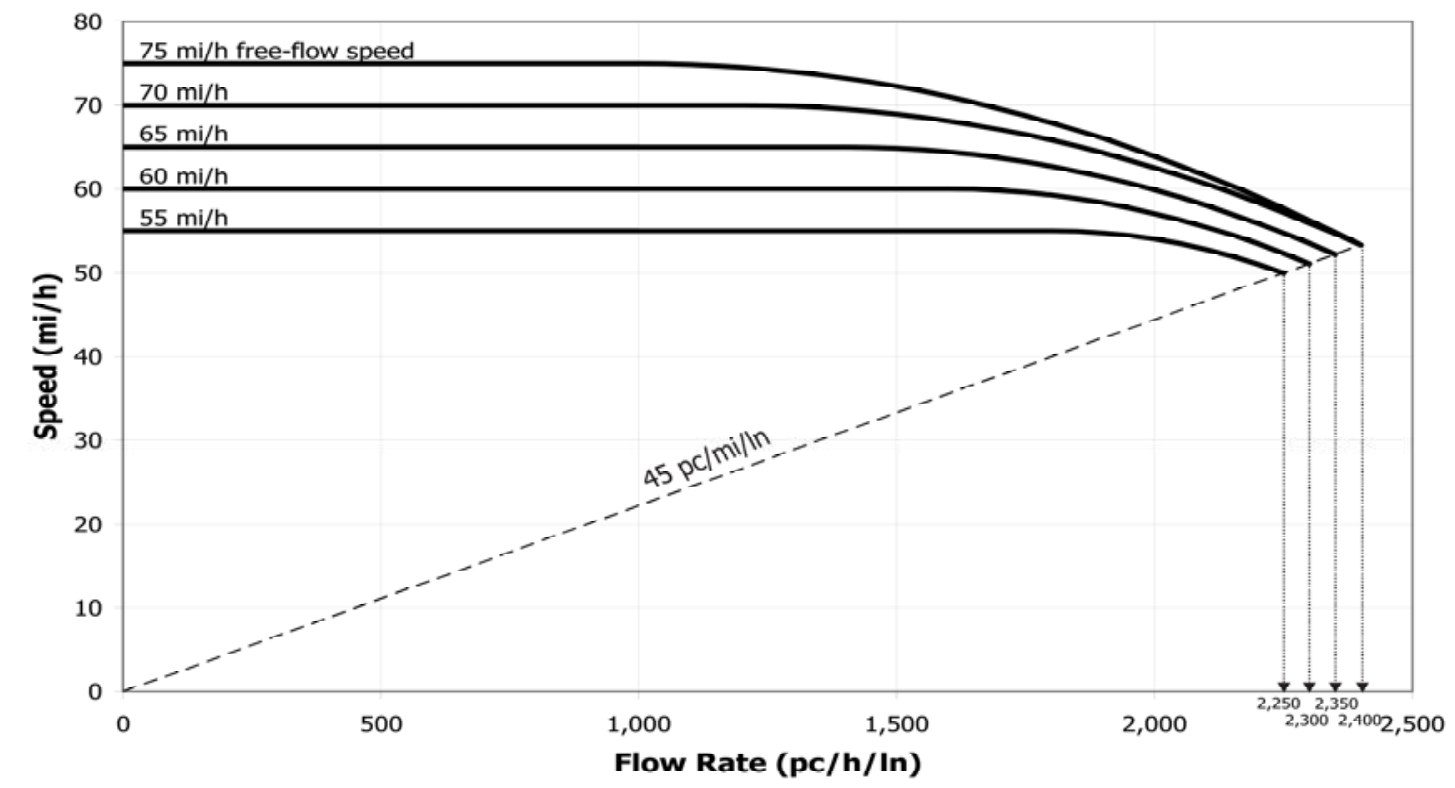

Figure 3-9: Speed-Flow curves for basic freeways segments under base conditions

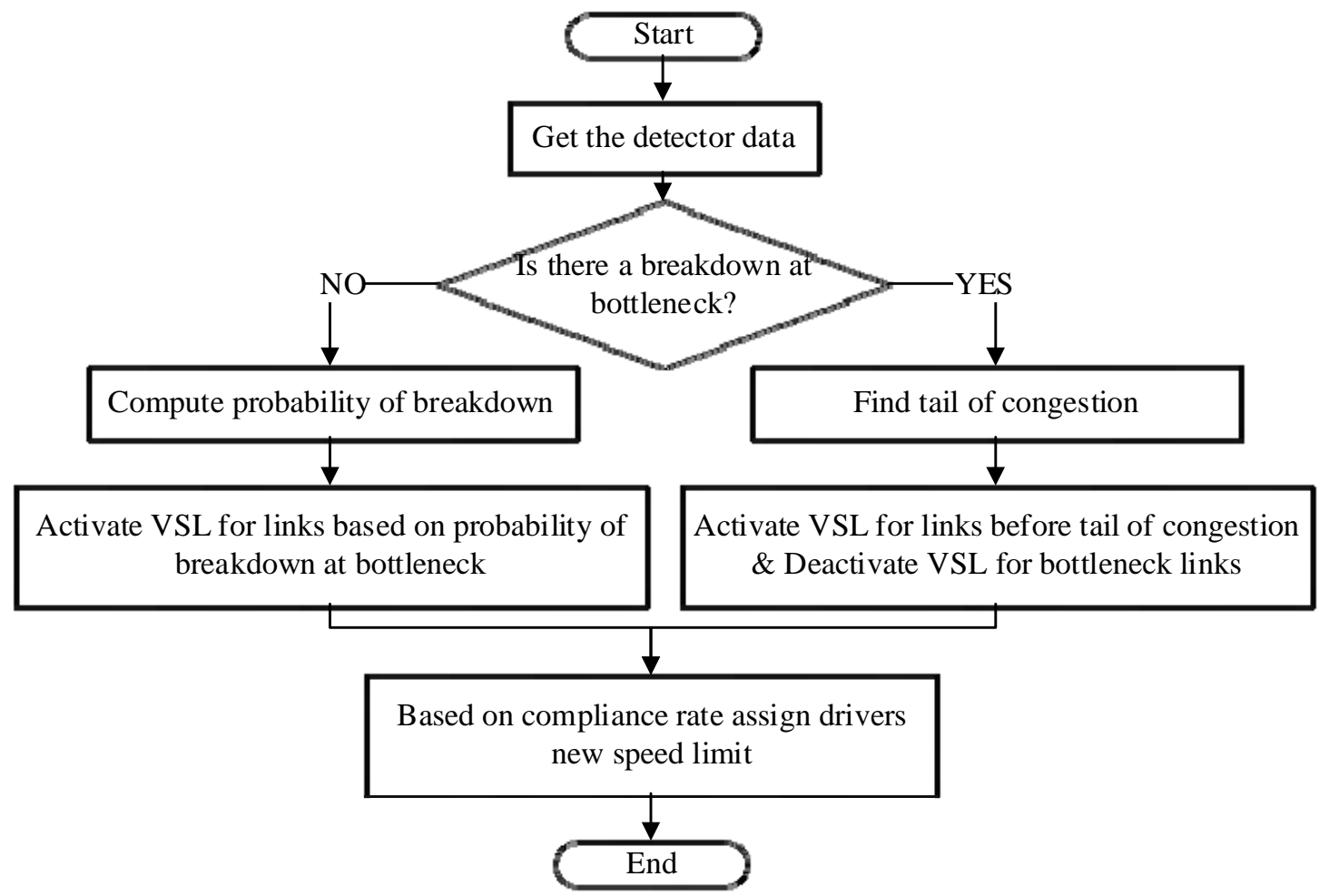

Figure 3-10: VSL strategy 
In summary, after traffic breakdown, the VSL strategy can be implemented to: (1) reduce the inflow of traffic and thus reduce the propagation of the congestion shockwave; and (2) increase the bottleneck capacity by deactivating the VSL at the head of the congestion.

\subsection{VSL Strategy Based on Infrastructure Detector Data}

This research proposes a shockwave-based VSL strategy and assesses its ability to reduce the probability and impacts of traffic flow breakdown at bottlenecks. The term "shockwave strategies" indicates that the influence area of VSL signs are pushed upstream of the bottleneck as the probability of the breakdown increases before breakdown, and as the back of queue propagates upstream after breakdown. The proposed system is based on recognizing the different influences of VSL before and after breakdown occurrence, as stated above.

This proposed VSL system is a reactive system that uses a heuristic switching logic-based controller with specified thresholds of prevailing traffic flow conditions. The logics used to switch the speed limits in the VSL systems, as reported in the literature, utilize thresholds based on occupancy, volume, speed, and combinations of these three variables. Based on simulation results, Elefteriadou et al. (2012) emphasized that improper selection of the thresholds can cause negative impacts on traffic conditions. Thus, finding the best set of thresholds for setting the speed limits is important.

In this research, occupancy data from point detectors located at and upstream of the bottleneck is used in switching between dynamic speed limit values. By using the fundamental diagram, the breakdown probability model, it is possible to identify a range 
of potential occupancy thresholds that define the separation between different traffic regimes, which can be used as initial values for VSL switching thresholds. The traffic is categorized in three regimes based on the fundamental traffic breakdown and the probability of breakdown, as follows: free-flow conditions, light congestion conditions, and heavy congestion conditions. These regimes are then associated with different speed values, and as initial values to be displayed by the VSL system. Then, using the developed simulation model, these thresholds are further fine-tuned using an exhaustive enumeration on the threshold ranges to find the set of thresholds that produce the best results in terms of traffic mobility in the network. To prevent fluctuation of the posted speed limits, the switching thresholds that lower speed limits are set to be different from those that switch to higher speed limits.

In the proposed VSL system, the posted speed limits are discrete and are selected in increments of $5 \mathrm{mph}$, as normally implemented in the real-world. For safety and driver compliance reasons, the VSL system is constrained to maximum updating frequencies, both in time and space.

As stated earlier, the theory and application of VSL before and after breakdown are different, discussed separately in the following section. Before breakdown, the goal of the VSL is to postpone or avoid breakdown occurrence. The VSL system is activated upstream of a potential bottleneck, which is a location with a high probability of breakdown based on traffic detectors upstream of the bottleneck. In the initial implementation, the location of the VSL is set at fixed distances, resulting in a fixed length of the VSL influence areas. Many researchers, such as Talebpour et al. (2013), indicated that finding the optimal location of the speed limit sign for this operation is 
important and has the most effect on system performance. In this study, an exhaustive enumeration is conducted to determine the optimal location of the fixed speed limit sign upstream of the bottleneck, taking into consideration the geometric constraints of the interchanges upstream of the bottleneck. When the VSL is placed further upstream of the potential bottleneck, the probability of breakdown is expected to further decrease. However, during uncongested conditions, this slows down vehicular speeds on longer sections of the freeway and can induce new bottlenecks due to lower capacities resulting from lower speeds. The optimal location is one that achieves the best balance between these two factors, which was obtained based on the simulation results.

An extension of the above method was conducted to determine if activating the speed limit signs further upstream of the optimal sign location is beneficial when an increase in occupancy and thus an increase in the probability of breakdown, is detected prior to reaching the high congestion levels that defines breakdown conditions. This approach is referred to as the "shockwave approach" and results in activating VSL signs further upstream when the traffic is recognized to have the potential to be on its way to breakdown. The proposed shockwave VSL system at this stage can analyze detector data from locations upstream of the bottleneck to determine if there is some increase in occupancy and will activate the upstream signs accordingly.

After breakdown, the VSL influence area should start at a sufficient distance upstream of the back of the queue to reduce shockwave propagation. In this study, the VSL influence extends from the first upstream VSL sign location to the location where the VSL is deactivated by a downstream VSL sign. When the VSL influence area is already congested, the reduction in speed limit by the VSL system has no effect on 
congestion. Thus, the proposed VSL system uses detector data upstream of the bottleneck to determine how far the congestion propagates upstream of the bottleneck, and furthermore, how far upstream of the back of queue the VSL signs need to be activated. In this approach, the location of the activated VSL sign is dynamic rather than static, with the location of the first activated sign pushed further upstream as the queue length grows; hence, it remains upstream of the back of the queue. This ensures that the VSL decreases the inflow traffic to the congested area in order to control the growth of the congestion. Again, this method of pushing the VSL location upstream is referred to as the shockwave approach in this study. In addition, the VSL system is deactivated within the congested area in order to encourage vehicles to accelerate if they can as they approach the head of the congested area.

The VSL system requires that traffic detector data be collected during short time intervals, such as 20-second to 1-minute intervals. Noise in the detector data causes fluctuation in the posted speed limit, which reduces the safety and compliance rates in real-world implementations, due to too many speed changes. These changes also lead to disturbance in traffic, which could result in breakdown. To reduce and avoid the impacts of noise, detector data should be smoothed. Two smoothing methods are tested in this study: the simple moving average method, and the exponential moving average method. The simple moving average is the average of the previous " $m$ " data points, where $m$ specifies the length of the rolling period. The second type of smoothing, the exponential moving average method, is described in the following equation:

$$
Y_{t}=\alpha X_{t}+(1-\alpha) Y_{t-1}
$$

where $X_{t}$ represents the measurement $\mathrm{t}$ timestamp and $Y_{t}$ is the smoothed traffic 
parameter at the $\mathrm{t}$ timestamp. The symbol $\alpha$ refers to a smoothing factor. The expression for $\alpha$ is shown below:

$$
\alpha=1-e^{\frac{-\Delta t}{\tau}}
$$

where $\Delta t$ is the time interval between two consecutive records and $\tau$ is a time constant. The commonly used value of $\alpha$ is 0.4 (Shen, 2008).

\subsection{VSL Strategy Based on Connected Vehicle Data}

With the fast-paced growth in technology, speed limit information can be disseminated to drivers by the dynamic exchange of messages between vehicles and infrastructure utilizing Connected Vehicle technology. In this research, the effectiveness of VSL signs is compared with the use of V2I communications to inform drivers about speed limits.

In this research, the functions and goals of VSL strategies based on Connected Vehicle data are the same as the VSL strategies based on Infrastructure detector data. The differences are the source, details, and types of the collected traffic data and the flexibility of where specific information is provided to the vehicles without being constrained by the VSL sign locations. With Connected Vehicles, information from equipped vehicles provides trajectory speed and the location of the vehicle, at each time step.

Collected speed data from Connected Vehicles can be aggregated across any freeway segment in time and space based on the application requirements. Aggregated speed data across freeway segments can present a clear picture of the current traffic conditions at different locations of the freeway. In this research, speeds are aggregated to 
find the locations of congestion in the network using speed data to justify disseminating new speed limits at a given location.

The locations of congestion can be found using thresholds on speed. Whenever the speed at one segment is above a specific threshold and the speed upstream of the segment is less than the threshold, that point is determined to be at the end of queue or congestion. Having a threshold on the speed difference between two neighboring locations can be used to define the beginning of the congested area. However, vehicle trajectories tend to be noisy due to various reasons, such as driver differences or the existence of traffic oscillations in congestion. Although this study uses aggregated speed data and has a lower amount of noise compared to individual vehicle data, as explained in Section 3.5., wavelet transform is used to automate the identification of breakdown occurrence, as well as the head and the tail of congestion. If the wavelet transform energy is not at its peak at the bottleneck area, the breakdown has not occurred yet. After breakdown occurs, the wavelet transform energy's peak can be used to identify the tail of the congested area.

In Connected Vehicle-based VSL, the VSL signs at different segments of the freeway are activated based on congestion location identified from speed trajectory data. Figure 3-8 shows the spatial distribution of aggregated trajectory data. As shown in this figure, using trajectory data, traffic regimes can be defined either based on predefined speed thresholds or sharp changes in speed data. The location of congestion can be further determined from these identified traffic regimes. As explained in the VSL algorithm section, three different traffic regimes are considered to assign speed limit. It can be seen in Figure 3-11 that traffic is under free-flow conditions from the tail of the 
congested area to the tail of the VSL influence area, while traffic is in heavy congestion conditions from the bottleneck location to the location that speed begins to increase. The regime between these two locations is characterized as a light congestion condition.

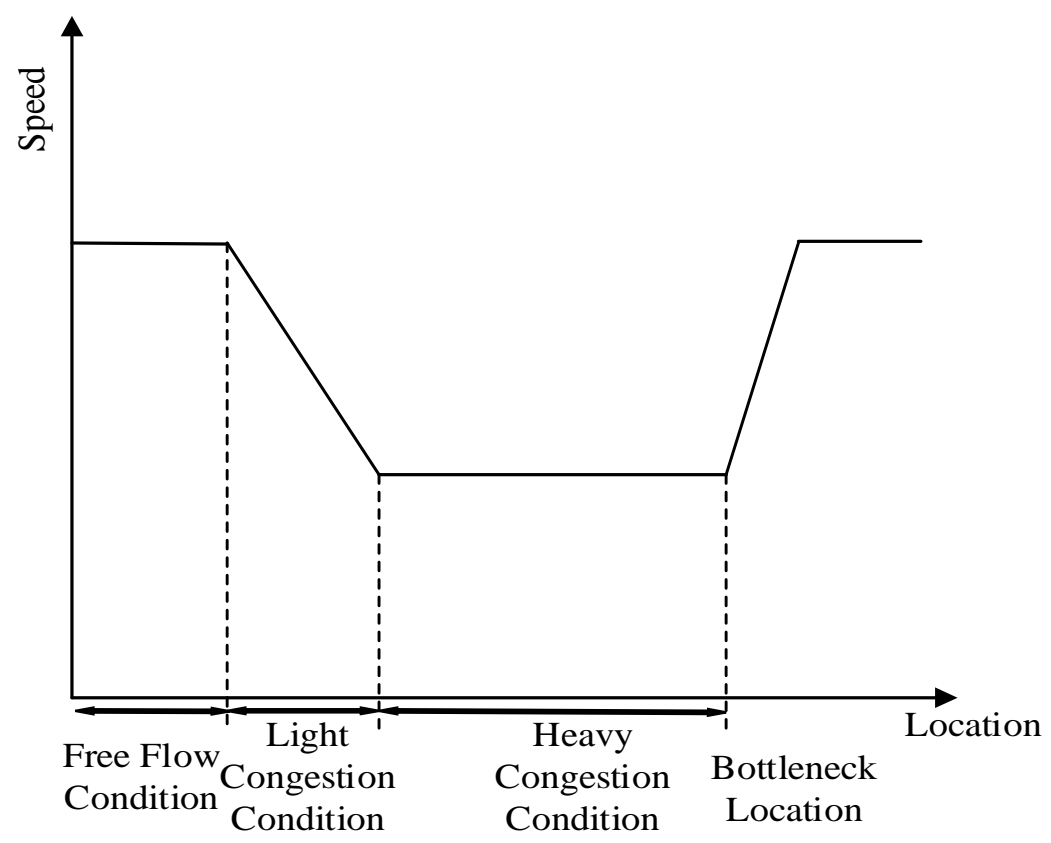

Figure 3-11: Traffic Regimes at Congested area

A shockwave-based VSL implementation is developed based on Connected Vehicle data, which is similar to the aforementioned VSL based on infrastructure detector data. Whenever the tail of congestion grows and gets close to the tail of the VSL influence area, one segment whose length is about one-third of a mile based on the geometric design of the facility is added to VSL influence area. Whenever the tail of the shrinking congestion and VSL influence area become far enough from each other, the VSL is deactivated for the last segment. As mentioned before, for safety and driver compliance reasons, the VSL system is constrained to a maximum change of speed limit, both in time and space. 


\subsection{Wavelet Transform}

Identifying sharp changes in the speed data is an important issue in this research for two reasons: 1) to identify the starting time of breakdown and its duration, 2) to find the location of congestion using trajectory speed data. Wavelet transform is capable of identifying sharp changes in non-stationary data (Meyer and Salinger, 1995).

A wavelet, $\psi(t)$, is a real or complex mathematical function. Wavelets can be categorized as discrete and continuous. Discrete wavelets are more efficient than continuous wavelets due to their ability to inverse the transform procedure to obtain data without noise (Adeli 2008). However, in this study we use continuous wavelets because we do not aim to analyze data with filtered noise, and furthermore, the continuous wavelet is more accurate. The general formulation of continuous wavelet transform (CWT), which is a wavelet transform coefficient (output) of a continuous signal $\chi(t)$, is:

$$
T(\alpha, \beta)=w(\alpha) \int_{-\infty}^{\infty} \chi(t) \psi\left(\frac{t-\beta}{\alpha}\right) d t
$$

where $\alpha$ is the scale parameter, and $\beta$ is the translation parameter. Scale parameter controls dilation and contraction of the wavelet, and translation parameter is about controlling the movement of the wavelet along the time dimension. The $w(\alpha)$ is a weighting function that normalizes the energy at all scales. This function is typically

considered to be $\frac{1}{\sqrt{\alpha}}$. In this research, velocity, $v(t)$, is considered a continuous signal. Note that whenever $\alpha=1$ and $\beta=0$, wavelet function is called "mother wavelet." There are different popular wavelet families, such as Haar, Daubechies, Meyer, Gaussian, Mexican hat, Morlet, and Coifman. Finding the optimal mother wavelet is not important in practice since all provide similar results, which are almost optimal (Donoho, 1993). 
Like Zheng et al. (2011), the Mexican hat wavelet, as defined in Equation (3-14) and shown in Figure 3-12, was selected in this study to analyze traffic data. The Mexican hat mother wavelet is the second derivative of the Gaussian distribution function, $e^{-\frac{t^{2}}{2}}$.

$$
\psi\left(\frac{t-\beta}{\alpha}\right)=\left(1-\left(\frac{t-\beta}{\alpha}\right)^{2}\right) e^{-\left(\frac{t-\beta}{\alpha}\right)^{2}}
$$

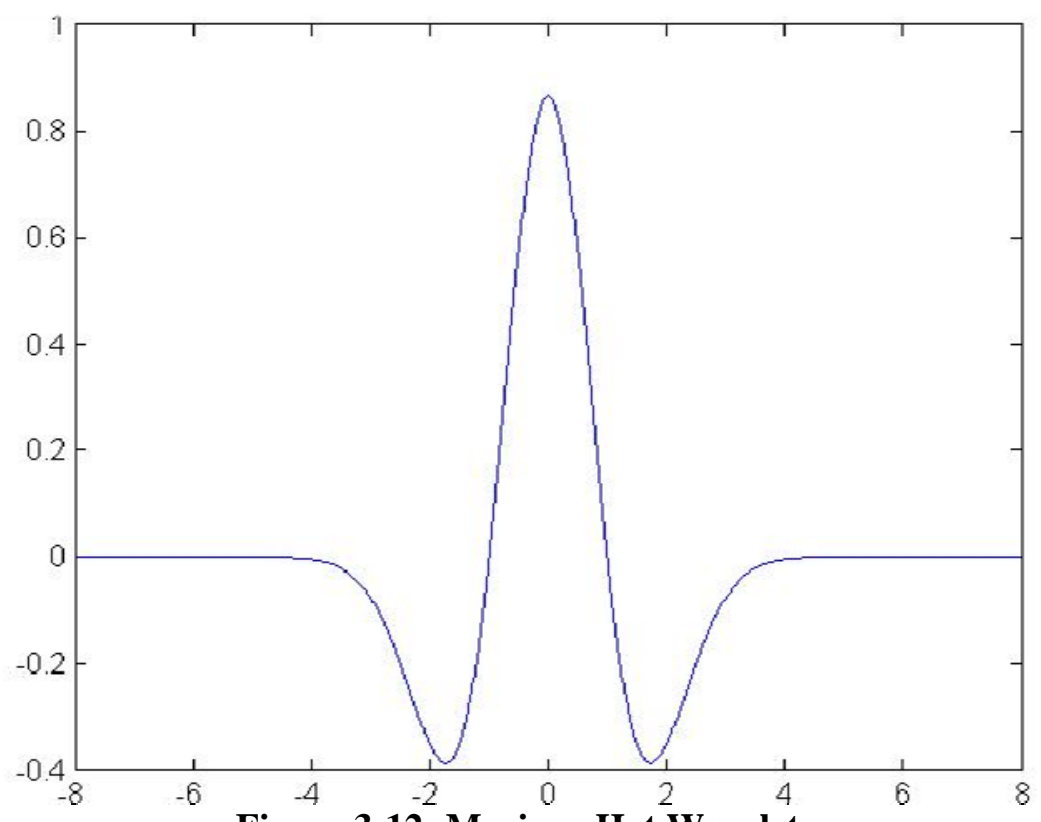

Figure 3-12: Mexican Hat Wavelet

By plugging (3-14) into (3-13), a wavelet transform of speed, $v(t)$, can be formulated as follows:

$$
T(\alpha, \beta)=\frac{1}{\sqrt{\alpha}} \int_{-\infty}^{\infty} v(t)\left(1-\left(\frac{t-\beta}{\alpha}\right)^{2}\right) e^{-\left(\frac{t-\beta}{\alpha}\right)^{2}} d t
$$

In specific $\alpha$ and $\beta$, signal energy is defined as follows:

$$
E_{\alpha, \beta}=|T(\alpha, \beta)|^{2}
$$

A plot of $E_{\alpha, \beta}$ is known as a scalogram. The scalogram can be integrated across $\alpha$ or $\beta$, or across both to produce total energy. The average wavelet energy at $\beta$ can be 
computed by averaging wavelet transform coefficients for different scales using the admissibility constant, as follows:

$$
E_{\beta}=\frac{1}{\max (\alpha)} \int_{0}^{\infty}|T(\alpha, \beta)|^{2} d \alpha
$$

The fact that $E_{\beta}$ is based on wavelet transform across different scales rather than the most dominant ones makes wavelet transform an effective tool for analyzing the speed data at a bottleneck. Wavelet energy increases when there is a sharp change in the speed data.

$\beta$ is usually selected based on the time resolution of the original signal. The maximum value of $\alpha$ should be controlled by the boundary effect in order to capture details of the original signal. The boundary effect can be identified by large wavelet transform coefficients at end of the signal range. This effect exists because the signal range is infinite, and the external range is assumed to be zero. In other words, at the boundaries of a signal range, there is change from zero to an actual value, which leads to large wavelet coefficients. As the value of $\alpha$ increases, a longer duration of signal is being affected by the boundary effect, which means that the maximum value of $\alpha$ should be small enough such that a considerable portion of the signal is not affected by this effect. A common way to avoid this effect is to consider more data and extend a signal's range, and not consider the additional data in wavelet transform coefficients.

\subsection{Compliance Rate}

One of the most important issues in implementing VSL systems is driver behavior, and whether drivers will obey the speed limit signs. There have been VSL implementations such as the one on I-4 in Orlando, Florida that have not been successful 
and effective because drivers were not complying with the reduced speed limits (PBS\&J, 2009). Most researchers have not considered the compliance rate when assessing the effectiveness of VSL in simulation models. However, the effectiveness of a VSL system is dependent on the driver's compliance with the system. In addition, low compliance rates may result in negative effects on traffic flow. Piao et al. (2008) indicated that with low compliance rates, there is the possibility of large variations in speed. However, Talebpour et al. (2013) indicated that $10 \%$ of compliance with the VSL is sufficient to achieve the desired outcomes.

For vehicles in congested sections, the compliance rate is expected to have less of an effect on system performance since there is less opportunity for vehicles desiring higher speeds to overtake slower vehicles. However, when the posted speed limit changes at the less congested sections, some vehicles will comply with this new speed limit and decrease their speeds. The vehicles that do not comply with the VSL will continue at their speeds if they can. Otherwise, they follow the vehicles in front of them and decelerate like the leading vehicles. In this study, the impacts of compliance rates are investigated using the CORSIM Run-Time Extension (RTE) facility. A code was written in a format accepted by this facility for this purpose.

\subsection{Evaluation of VSL Strategies}

Traffic simulation is a valuable tool for analyzing and assessing ADTM strategies like VSL. The proposed VSL strategies are tested using the CORSIM microscopic traffic simulation model, with the VSL strategy logic incorporated in a dynamic link library (DLL). DLL is imported through the CORSIM Run-Time Extension (RTE) facility. It 
interfaces with the CORSIM simulation in real simulation running time. Building a CORSIM RTE requires a suitable compiler. Microsoft Visual C++ was used to create and compile the RTE in this study. The C++ Run-time extension header file that contains the export definitions was created to make CORSIM variables available. A flowchart with the general logic of the program is shown in Figure 3-13.

Upon the initialization of the simulation, the DLL program identifies which detectors are used to control the VSL system and the links affected by the VSL system. In addition, it determines how data are aggregated from the detectors.

After the initialization is complete, the DLL is accessed at the call point PREFRESIMVEHICLE. This occurs every time-step (one second) during the simulation before vehicle movements take place. If the simulation is not within the initialization period, in which the simulated network is filled up with vehicles, the current VSL values at each location are determined based on the average occupancy value relayed from the specified detectors in the infrastructure-based approach or trajectory speed data at specific segments upstream of the bottleneck in the Connected Vehicle-based approach. If it is determined that a speed change is needed, the free-flow speed is updated on the simulated links in the VSL influence area. The developed code of VSL can be found in Appendix A.

In the developed code, drivers will receive information about speed limits, depending on their location. The main difference between the dissemination speed limits through VSL signs and V2I communications is which driver receives the new speed limit information. If the speed limit of the segment is changed while a driver is already in the segment, driver will not be able to receive new information through VSL sign, as the VSL 
sign is not visible to him/her. However, with Connected Vehicle Technology, drivers are able to receive new posted speed limit even if they are in the middle of the segment.

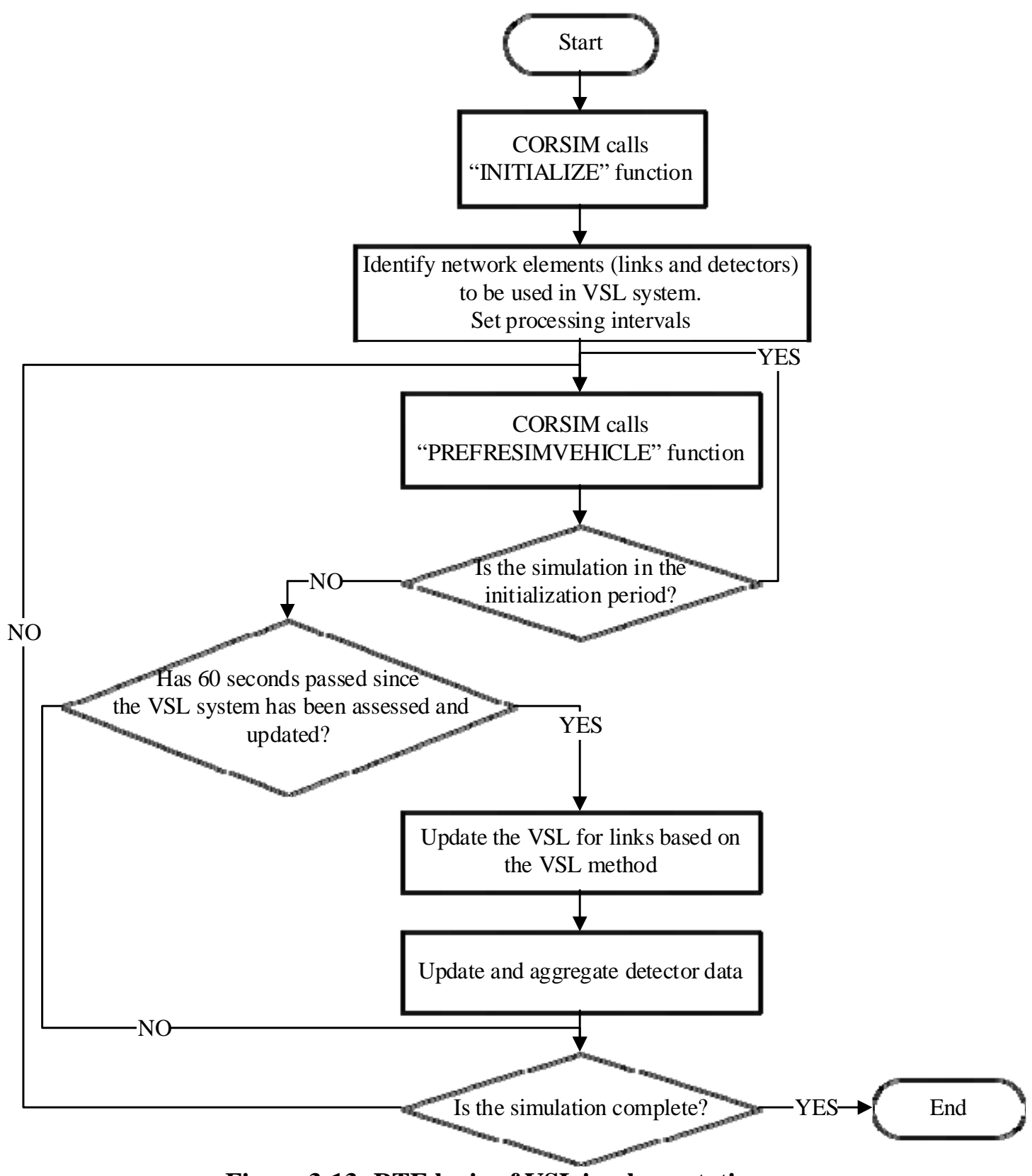

Figure 3-13: RTE logic of VSL implementation

It is also assumed in the developed code that when drivers receive new speed limit information, they may or may not comply with the updated speed limit, depending on the compliance rate. For a given compliance rate, drivers are randomly selected and the new 
speed limit information are only transferred to the selected drivers.

\subsection{Summary}

This dissertation develops a shockwave-based VSL system, which uses a heuristic switching logic-based controller with specified thresholds of prevailing traffic flow condition locations. This VSL strategy aims to improve mobility at recurrent bottlenecks. Before breakdown occurrence, the proposed VSL tries to postpone breakdown by decreasing the inflow and achieving uniform distribution in speed and flow. After breakdown, the VSL system aims to dampen the congestion by reducing the traffic inflow to the congested area and increasing the bottleneck capacity by deactivating the VSL at the head of the congested area. A shockwave-based VSL system is proposed to be evaluated that pushes the VSL location upstream as the congested area propagates upstream. In addition, Connected Vehicle technology provides a dynamic exchange of messages between vehicles and infrastructure, which allows the VSL system to have access to much more detailed traffic data. This dissertation investigates the effect of using Connected Vehicle data instead of detector data on VSL system performance. In this system, wavelet transform is used to analyze aggregated individual vehicles' speed data to determine the location of congestion.

The developed VSL algorithms are assessed using a CORSIM microscopic simulation model. Without calibration of the simulation model, there is no assurance that the model's outputs are reliable or that the model will correctly predict the traffic performance for the projects as a result of improvements. Even though traffic simulation models have been widely and increasingly used in the transportation field due to their 
cost-effectiveness, the calibration of simulation models is generally based on the capacity, volume and system performance values, which does not take the traffic breakdown characteristics into consideration. However, since the proposed VSL strategies are countermeasures to the impacts of breakdown conditions, inclusion of the breakdown characteristics in the calibration procedure is important in order to obtain a reliable assessment. Several enhancements are proposed in this study, including using the wavelet transform to determine the start and end times of breakdown occurrence, as well as to account for the breakdown characteristics at bottleneck locations in the calibration process. 


\section{CHAPTER 4}

\section{DATA ACQUISITION AND PREPROCCESSING}

This research aims to examine the developed VSL strategy. The study area and field data used in this study are first described in this chapter, followed by a detailed discussion of breakdown characteristics at the bottlenecks within the study area. The effort required the collection of connected vehicle data, which is then presented.

\subsection{Study Corridor}

The case study network used in this research is a 12-mile segment of the I-95 northbound freeway facility in Miami, Florida in the PM peak period. The network is shown in Figure 4-1. The locations of the bottlenecks in the PM peak are highlighted in this figure. The starting point of the network is located on the I-95 mainline at NW $8^{\text {th }}$ Street, and the ending point is located on I-95 at NE $187^{\text {th }}$ Street. In this research, the simulation model for the PM peak period is from 3:00 PM to 6:00 PM.

\subsection{Infrastructure Detector Data Acquisition and Preprocessing}

Infrastructure detector data collected by the Florida Department of Transportation (FDOT) District 6 Traffic Management Center (TMC) is extensively used in this project for model calibration and validation. Microwave detectors are installed by FDOT every 0.3 to 0.5 mile in the corridor to report volume, speed, and density measurements in 20-second intervals for each lane. This data were obtained from the Statewide Transportation Engineering Warehouse for Archived Regional Data (STEWARD). The STEWARD database contains aggregated traffic detector data (traffic volumes, speeds, 
and occupancies) by 5-, 15-, and 60-minute periods. The STEWARD database is available through a web-based interface. In the network of this case study, 38 true presence microwave detectors are installed, providing 20-second traffic data, including speed, volume, and occupancy measurements. In addition, the FDOT Statistics Office collects portable traffic monitoring sites (PTMS) 15-minute ramp counts. The PTMS data is collected for two or three days per year and includes truck percentages and volumes. This data is used to supplement the ITS data that does not include ramp volume. Furthermore, ramp metering data were obtained from the FDOT District 6 TMC.

To identify the locations of the recurrent bottlenecks, 14 days were chosen as representatives of normal days on the corridor. In this research, a normal day is defined as a weekday, with a PM peak period that is free of incidents, special events, and weather events in the study area. To highlight the variation between the congestion levels for the selected normal days, a congestion index is calculated as the mean relative difference of field speed against free-flow speed at all freeway segment locations during the study period, as follows:

$$
C I=\frac{1}{N \times T} \sum_{t} \sum_{i} \frac{S_{F F S, i}-S_{i, t}}{S_{F F S, i}} \quad \forall S_{F F S}>S_{i, t}
$$

where

$$
\begin{aligned}
C I & =\text { congestion index, } \\
S_{F F S, i} & =\text { free-flow speed for segment } i, \\
S_{i, t} & =\text { speed at segment } i, \text { at time interval } t, \\
N & =\text { total number of segments, and } \\
T & =\text { total number of time intervals. }
\end{aligned}
$$




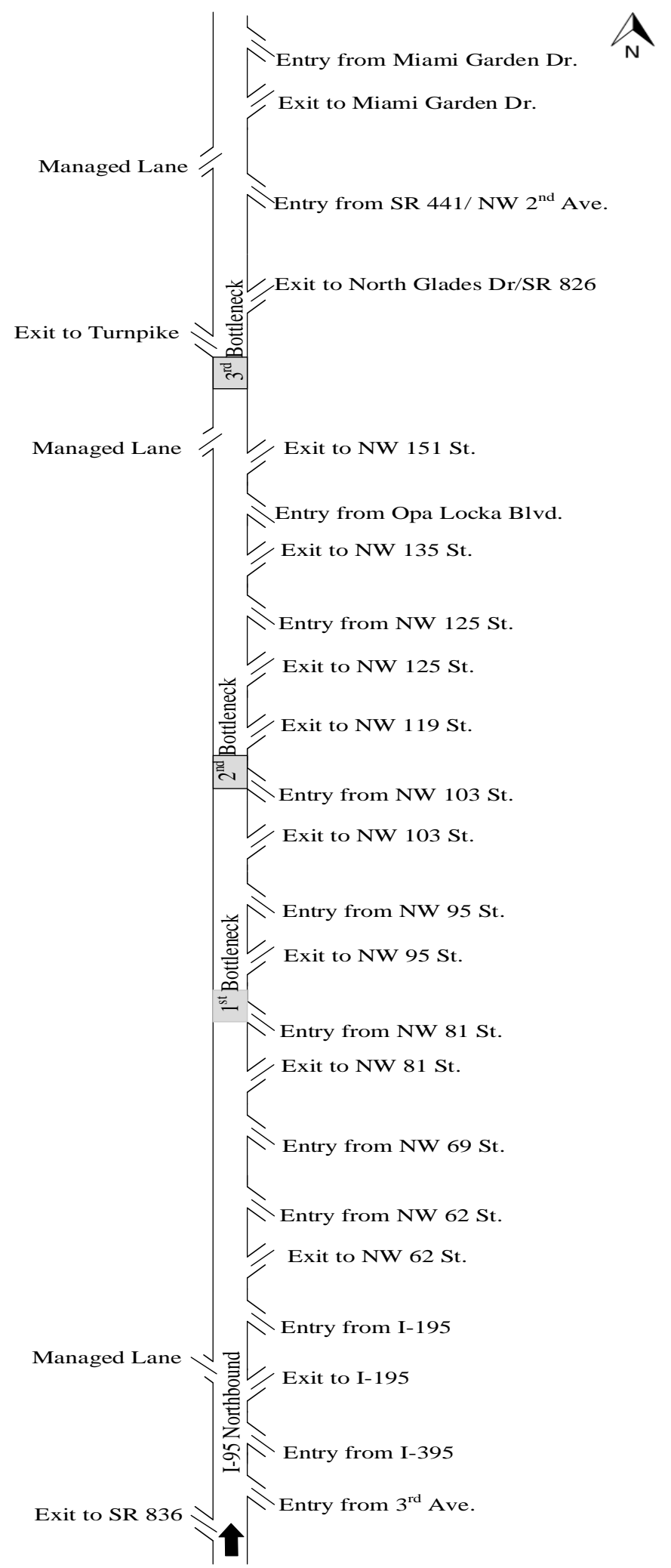

Figure 4-1: Study area 
In this study, the congested areas and bottleneck locations were identified utilizing time-space speed contour maps created based on detector data of normal days during the PM peak. It was possible to identify the three locations highlighted in Figure 4-1, as bottleneck locations. Based on the congestion index values and visual audit, three days were selected to represent three traffic congestion levels: heavy, medium, and light. Figure 4-2 shows the time-space speed contour maps for these three days. These figures use detector data aggregated over a one-minute period. The red regions correspond to low speeds, according to the scale given in the legend. In this way, the speed contour plot clearly shows the locations of the bottlenecks and associated queue built-up and dissipation.

Two of the three bottlenecks are located immediately downstream of merging on-ramps. The third is caused by a spillback from an off-ramp to Florida's Turnpike, a major limited access facility in the region. To reduce the congestion at the first two bottlenecks caused by merging on-ramps, a fuzzy logic-based ramp metering strategy was implemented, operating in the northbound direction. The implemented ramp metering strategy is shown, based on before-after assessments, to improve system performance. However, as can be seen in Figure 4-2, the current implementation of the ramp metering is not able to eliminate the identified three bottlenecks.

In order to determine the various breakdown characteristics at each bottleneck, it was necessary to estimate the breakdown starting time and the duration of breakdown. This research uses the definition presented by Elefteriadou et al. (2011), and the wavelet transform method is used to identify the start and end times of the breakdown. The rest of the estimates are based on this time window. The results show that using each of these 
two methods leads to similar results. Figure 4-3 presents the calculation of the wavelet energy of speed data at the bottlenecks.

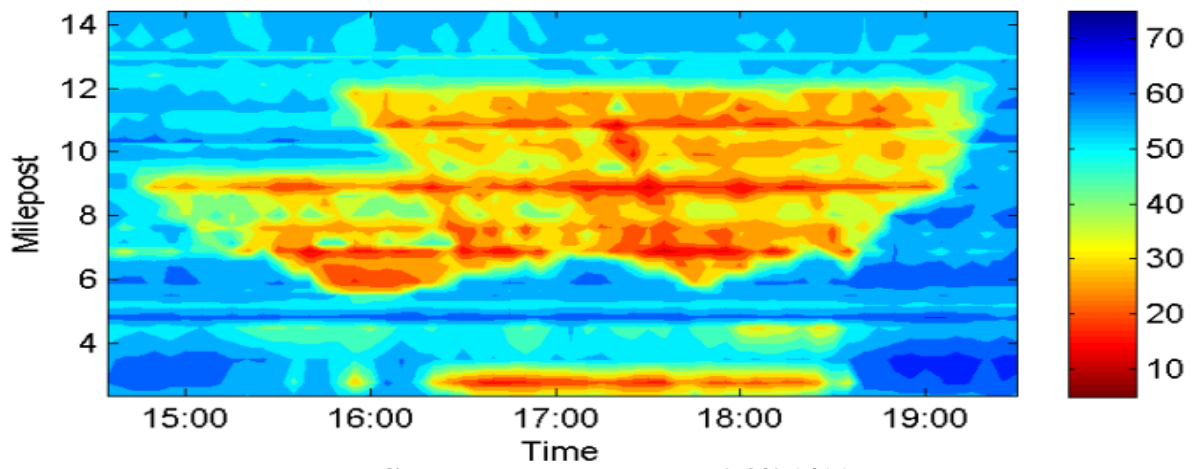

(a) Speed contour map- 04/01/11

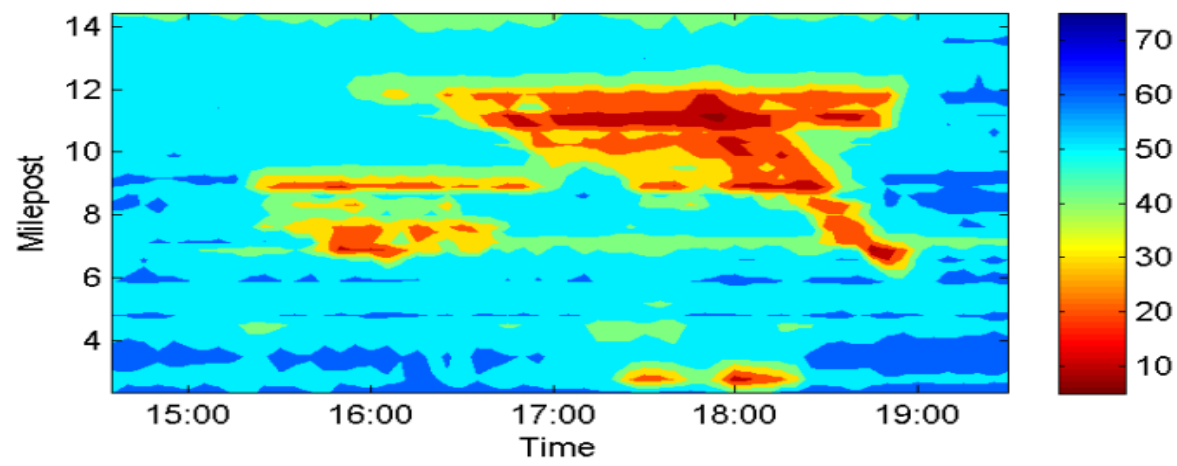

(b) Speed contour map- 05/20/10

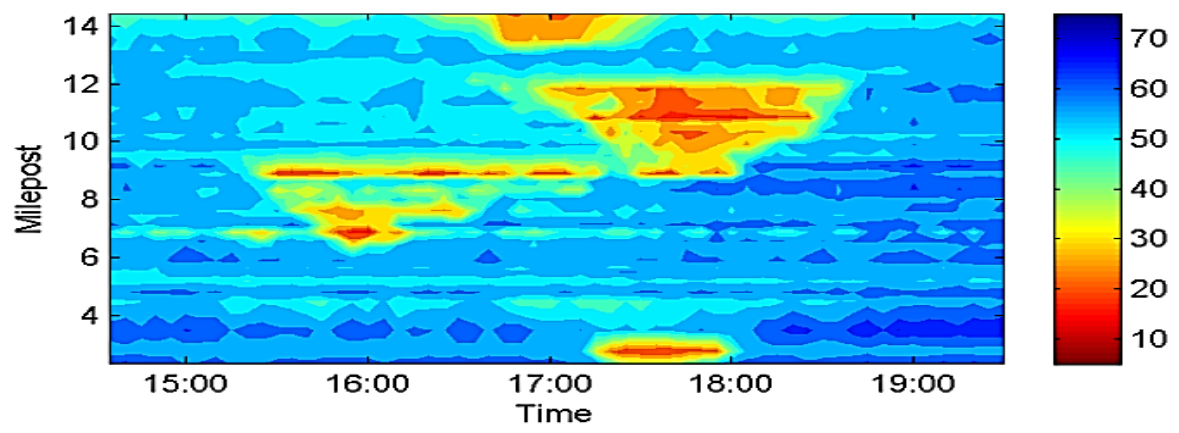

(c) Speed contour map- 05/12/10

Figure 4-2: Speed contour maps based on real-world data

Figure 4-3 (c) shows the temporal distribution of energy. The lighter regions of the contour represent larger values of the wavelet transform coefficients, which lead to 
higher wavelet energy. The peak of wavelet energy indicates the sharp change in the speed data. Figure 4-3 (d) shows wavelet energy for the computed speed time-series using Equation (3-17) and clearly indicates the start and the end of time breakdown.
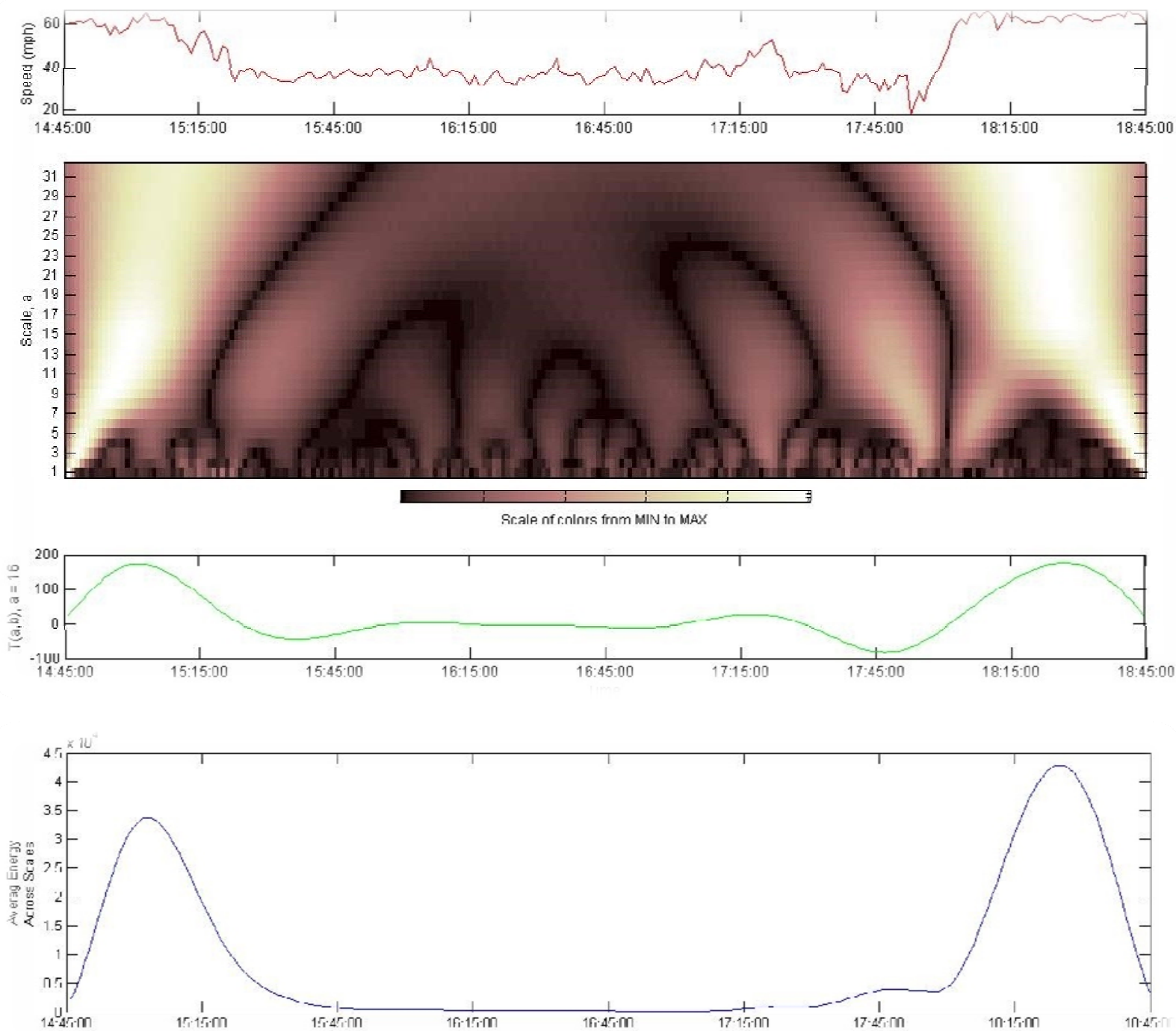

Figure 4-3: Illustration of wavelet transform and energy calculation. (a) Time series plot of speed at the second bottleneck at 05/12/10; (b) Contour map of the absolute values of wavelet transform coefficients, $|T(\alpha, \beta)|$, from scale $\alpha=1-32$; (c) WT coefficients, $T(\alpha, \beta)$, at scale $\alpha=16$; (d) The temporal distribution of average wavelet-based energy across scales.

When a breakdown occurs, congestion propagates and the shockwave starts to move to 
upstream locations. As a result, sometimes neighboring bottlenecks impact each other and merge together. For this reason, the duration of the breakdown at the upstream bottlenecks is calculated as much as possible for days with no or small impacts from downstream bottlenecks. Tables 4-1 to 4-3 present the various breakdown characteristics for the three bottlenecks based on real-world data for different days. These tables clearly show the difference between the pre-breakdown capacity and the queue discharge rate, and the significant variation in the breakdown occurrence and duration between days. It is also interesting to note that the queue discharge rate and the speeds prior to and after breakdown have similar values for different days.

Table 4-1: Breakdown characteristics at first bottleneck based on real-world data

\begin{tabular}{|c|c|c|c|c|c|c|c|c|}
\hline Date & $\begin{array}{l}\text { Starting } \\
\text { Time } \\
\text { (hh:mm) }\end{array}$ & $\begin{array}{l}\text { Duration } \\
\text { (hh:mm) }\end{array}$ & $\begin{array}{c}\text { Speed } \\
\text { Before } \\
\text { Breakdown } \\
(\mathrm{mph})\end{array}$ & $\begin{array}{l}\text { Pre-Breakdown } \\
\text { Flow } \\
\text { (veh/hr) }\end{array}$ & $\begin{array}{l}\text { Breakdown } \\
\text { Speed } \\
\text { (mph) }\end{array}$ & $\begin{array}{c}\text { Queue } \\
\text { Discharge } \\
\text { (veh/hr) }\end{array}$ & $\begin{array}{c}\text { Speed } \\
\text { After } \\
\text { Breakdown } \\
(\mathrm{mph})\end{array}$ & $\begin{array}{c}\text { Recovery } \\
\text { Flow After } \\
\text { Breakdown } \\
\text { (veh/hr) }\end{array}$ \\
\hline $5 / 12 / 10$ & 15:35 & 1:00 & 55.43 & 8076 & 31.1 & 6814 & 56.95 & 6888 \\
\hline $5 / 20 / 10$ & $15: 20$ & $2: 40$ & 55.39 & 7188 & 28.4 & 6571 & -*** & - \\
\hline 6/17/10 & $17: 20$ & $0: 40$ & 53.75 & 7644 & 30.01 & 6261 & - & - \\
\hline 10/6/10 & $15: 05$ & $2: 55$ & 55.8 & 6732 & 27.03 & 6375 & - & - \\
\hline 10/7/10 & $15: 30$ & $2: 30$ & 50.81 & 7584 & 29 & 6475 & - & - \\
\hline $11 / 4 / 10$ & $15: 45$ & $2: 15$ & 55.47 & 7632 & 28.1 & 6427 & - & - \\
\hline $11 / 16 / 10$ & $15: 40$ & $2: 20$ & 58.11 & 7092 & 32.44 & 6356 & - & - \\
\hline $11 / 18 / 10$ & $15: 05$ & $2: 25$ & 58.62 & 7452 & 27.58 & 6813 & 50.14 & 6696 \\
\hline 11/30/10 & $15: 25$ & 2:35 & 55.88 & 7620 & 28.08 & 6423 & - & - \\
\hline 1/18/11 & $15: 25$ & 2:35 & 57.97 & 7668 & 28.32 & 6535 & - & - \\
\hline 2/12/11 & $-*$ & 3:00 & 54.13 & 7836 & 27.76 & 6501 & - & - \\
\hline 3/15/11 & $15: 45$ & $2: 15$ & 57.19 & 8160 & 30.6 & 6594 & - & - \\
\hline 3/20/11 & $-* *$ & - & - & - & - & - & - & - \\
\hline $4 / 1 / 11$ & $15: 05$ & $2: 55$ & 55.22 & 7536 & 28.36 & 6369 & - & - \\
\hline Average & 15:35 & $2: 18$ & 55.67 & 7555 & 28.98 & 6501 & 53.55 & 6792 \\
\hline $\begin{array}{l}\text { Standard } \\
\text { Deviation }\end{array}$ & $0: 34$ & $0: 40$ & 2.01 & 371 & 1.52 & 160 & 3.41 & 96 \\
\hline
\end{tabular}

*: The breakdown started prior to 3:00 PM, starting time of this study's analysis.

**: The breakdown did not occur. 
***: The breakdown did not recover prior to 6:00 PM, ending time of this study's analysis.

Table 4-2: Breakdown characteristics at second bottleneck based on real-world data

\begin{tabular}{|c|c|c|c|c|c|c|c|c|}
\hline Date & $\begin{array}{l}\text { Starting } \\
\text { Time } \\
\text { (hh:mm) }\end{array}$ & $\begin{array}{l}\text { Duration } \\
\text { (hh:mm) }\end{array}$ & $\begin{array}{c}\text { Speed } \\
\text { Before } \\
\text { Breakdown } \\
(\mathrm{mph})\end{array}$ & $\begin{array}{l}\text { Pre-Breakdown } \\
\text { Flow } \\
\text { (veh/hr) }\end{array}$ & $\begin{array}{l}\text { Breakdown } \\
\text { Speed } \\
\text { (mph) }\end{array}$ & $\begin{array}{c}\text { Queue } \\
\text { Discharge } \\
\text { (veh/hr) }\end{array}$ & $\begin{array}{c}\text { Speed } \\
\text { After } \\
\text { Breakdown } \\
\text { (mph) }\end{array}$ & $\begin{array}{c}\text { Recovery } \\
\text { Flow After } \\
\text { Breakdown } \\
\text { (veh/hr) }\end{array}$ \\
\hline $5 / 12 / 10$ & $15: 25$ & 2:35 & 59.48 & 7380 & 36.69 & 6840 & $-* * *$ & - \\
\hline $5 / 20 / 10$ & $-*$ & 3:00 & 62.15 & 7200 & 36.25 & 6775 & - & - \\
\hline 6/17/10 & 16:20 & $1: 40$ & 62.23 & 7044 & 38.42 & 6870 & - & - \\
\hline 10/6/10 & 16:55 & 1:05 & 47.09 & 7128 & 33.82 & 6684 & - & - \\
\hline $10 / 7 / 10$ & $-* *$ & - & - & - & - & - & - & - \\
\hline $11 / 4 / 10$ & $15: 25$ & 2:35 & 54.10 & 7272 & 33.56 & 6689 & - & - \\
\hline $11 / 16 / 10$ & 15:30 & $2: 10$ & 56.02 & 7380 & 33.08 & 6808 & 50.29 & 6768 \\
\hline $11 / 18 / 10$ & - & 3:00 & 55.08 & 7296 & 33.68 & 6622 & - & - \\
\hline $11 / 30 / 10$ & $15: 20$ & $2: 40$ & 55.56 & 7152 & 34.33 & 6616 & - & - \\
\hline 1/18/11 & - & 3:00 & 57.31 & 7338 & 34.89 & 6823 & - & - \\
\hline 2/12/11 & - & 3:00 & 55.43 & 7548 & 35.13 & 6770 & - & - \\
\hline 3/15/11 & $15: 35$ & $2: 25$ & 56.58 & 7568 & 33.75 & 6572 & - & - \\
\hline 3/20/11 & - & - & - & - & - & - & - & - \\
\hline 4/1/11 & - & 3:00 & 56.83 & 7287 & 33.75 & 6653 & - & - \\
\hline Average & $15: 47$ & $2: 30$ & 56.49 & 7299 & 34.78 & 6727 & 50.29 & 6768 \\
\hline $\begin{array}{l}\text { Standard } \\
\text { Deviation }\end{array}$ & $0: 33$ & $0: 35$ & 3.80 & 152 & 1.53 & 95 & 0.00 & 0 \\
\hline
\end{tabular}

*: The breakdown started prior to 3:00 PM, starting time of this study's analysis.

**: The breakdown did not occur.

***: The breakdown did not recover prior to 6:00 PM, ending time of this study's analysis. 
Table 4-3: Breakdown characteristics at third bottleneck based on real-world data

\begin{tabular}{lcccccccc}
\hline \multicolumn{1}{c}{ Date } & $\begin{array}{c}\text { Starting } \\
\text { Time } \\
\text { (hh:mm) }\end{array}$ & $\begin{array}{c}\text { Duration } \\
\text { (hh:mm) }\end{array}$ & $\begin{array}{c}\text { Speed } \\
\text { Before } \\
\text { Breakdown } \\
\text { (mph) }\end{array}$ & $\begin{array}{c}\text { Pre-Breakdown } \\
\text { Flow } \\
\text { (veh/hr) }\end{array}$ & $\begin{array}{c}\text { Breakdown } \\
\text { Speed } \\
\text { (mph) }\end{array}$ & $\begin{array}{c}\text { Queue } \\
\text { Discharge } \\
\text { (veh/hr) }\end{array}$ & $\begin{array}{c}\text { Speed } \\
\text { After } \\
\text { Breakdown } \\
\text { (mph) }\end{array}$ & $\begin{array}{c}\text { Recovery } \\
\text { Flow After } \\
\text { Breakdown } \\
\text { (veh/hr) }\end{array}$ \\
\hline 5/12/10 & $17: 00$ & $1: 00$ & 55.43 & 9192 & 23.03 & 7596 & $-* * *$ & - \\
5/20/10 & $16: 20$ & $1: 40$ & 55.39 & 9024 & 23.50 & 7686 & - & - \\
6/17/10 & $16: 55$ & $1: 05$ & 53.75 & 8820 & 23.03 & 7778 & - & - \\
10/6/10 & $16: 15$ & $1: 45$ & 55.8 & 8676 & 24.67 & 7910 & 56.39 & 7632 \\
10/7/10 & $16: 30$ & $1: 30$ & 50.81 & 8796 & 25.65 & 8001 & - & - \\
11/4/10 & $16: 50$ & $1: 10$ & 55.47 & 8532 & 26.58 & 7727 & - & - \\
11/16/10 & $16: 45$ & $1: 15$ & 58.11 & 9024 & 23.26 & 7634 & - & - \\
11/18/10 & $16: 05$ & $1: 55$ & 58.62 & 8832 & 25.58 & 7684 & - & - \\
11/30/10 & $16: 30$ & $1: 30$ & 55.88 & 8382 & 26.08 & 7827 & - & - \\
1/18/11 & $16: 45$ & $1: 15$ & 57.97 & 8856 & 23.28 & 7759 & - & - \\
2/12/11 & $16: 30$ & $1: 30$ & 54.13 & 9228 & 23.42 & 7805 & - & - \\
3/15/11 & $16: 30$ & $1: 30$ & 57.19 & 8832 & 26.26 & 7859 & - & - \\
3/20/11 & $-* *$ & - & - & - & - & - & - & - \\
4/1/11 & $16: 05$ & $1: 55$ & 55.22 & 9240 & 25.51 & 7782 & - & - \\
\hline Average & $16: 32$ & $1: 27$ & 55.67 & 8880 & 24.60 & 7773 & 56.39 & 7632 \\
Standard & $0: 17$ & $0: 17$ & 2.01 & 251 & 1.32 & 108 & 0.00 & 0 \\
Deviation & & & & & & & & \\
\hline
\end{tabular}

*: The breakdown started prior to 3:00 PM, starting time of this study's analysis.

$* *$ : The breakdown did not occur.

***: The breakdown did not recover prior to 6:00 PM, ending time of this study's analysis.

When examining the characteristics of breakdown in Tables 4-1 to 4-3, it should be noted that the variations in day-to-day real-world performance in event-free days are due to the breakdown stochasticity. It can also be due to variations in traffic demands between days. Comparing breakdown speed at different bottlenecks, it can be seen that the breakdown speed at the third bottleneck is significantly less than other bottlenecks. As shown in these tables, the standard deviation of breakdown speed at each bottleneck 
during breakdown is about $1-2 \mathrm{mph}$. Thus, it is concluded that this breakdown characteristic remains almost constant at each bottleneck. The starting time of breakdown varies between different days. To show the extent of variability in pre-breakdown flows at different bottlenecks, the coefficient of variation is estimated. The coefficient of variance of pre-breakdown is almost $5 \%$ at the first bottleneck, $2 \%$ at the second bottleneck, and $3 \%$ at the third bottleneck. These variations clearly show the stochastic nature of capacity. The queue discharge, however, is a significantly less variable as it is the long-run average of flow during the breakdown period. Comparing pre-breakdown flow and queue discharge, the capacity drop after breakdown occurrence is almost $13 \%$ for the first bottleneck, $7 \%$ for the second bottleneck, and $12 \%$ for the third bottleneck. The speeds before and after the breakdown are considerably the same and are similar to the free-flow speed.

\subsection{Connected Vehicle Data Acquisition and Preprocessing}

Through Connected Vehicle technology, the Connected Vehicles that are equipped with On-Board Unit (OBU) are enabled to transfer trajectory data to roadside infrastructure. Since the field Connected Vehicle data are not available in this study or as part of this research, V2I communication is modeled in the micro-simulation using CORSIM Run-Time Extension (RTE) facility. In the CORSIM Run-Time Extension DLL file, the simulated vehicle trajectory data are extracted at a resolution of 1 second, which is considered the available Connected Vehicle information and used in the latter steps. The obtained speed data from such information can be aggregated across any freeway segment in time and space based on the application requirements. Aggregated speed data 
across freeway segments can present a clear picture of the current traffic conditions at different locations of the freeway. In this research, speeds are aggregated to find the locations of congestion in the network. However, other than using the detailed collected data in this way, no attempts were made to utilize other types of collected parameters to enhance the algorithm developed based on infrastructure detector data. As the percentage of vehicles that are equipped with on-board units and market penetration increases, the accuracy of the collected data increases as well.

At each time step (1 second), the individual vehicle's data, speed and location were collected from the simulation runs. As mentioned before, the trajectory speed data is being aggregated across any freeway segment in time and space. For this purpose, the segment length should be chosen such that all the important information required for the application is captured and the noise in data is reduced. In addition, the smaller segment length means the availability of more data, which results in more computational time. Since the VSL strategy is constrained to maximum updating frequency both in time and space, it was found that aggregating trajectory speed data for less than 500 -foot segments cannot improve the performance of the VSL strategy.

The accuracy of the collected data depends on the market penetration, which is the portion of drivers equipped with on-board units. Data collected based on different market penetrations were compared to find the minimum market penetration that provides accurate data, in other words, the minimum sample size that can represent the population accurately. For this purpose, the accuracy of data with different market penetration were compared to that with $100 \%$ market penetration by using two statistical measurements: the Correlation Coefficient, as shown in Equation 3-8, and the Root Mean 
Squared Normalized Percent Error (RMSNPE), as shown in Equation 3-10. Figures 4-4 and 4-5 show the Correlation Coefficient and RMSNPE of speed estimates based on collected data under different market penetrations.

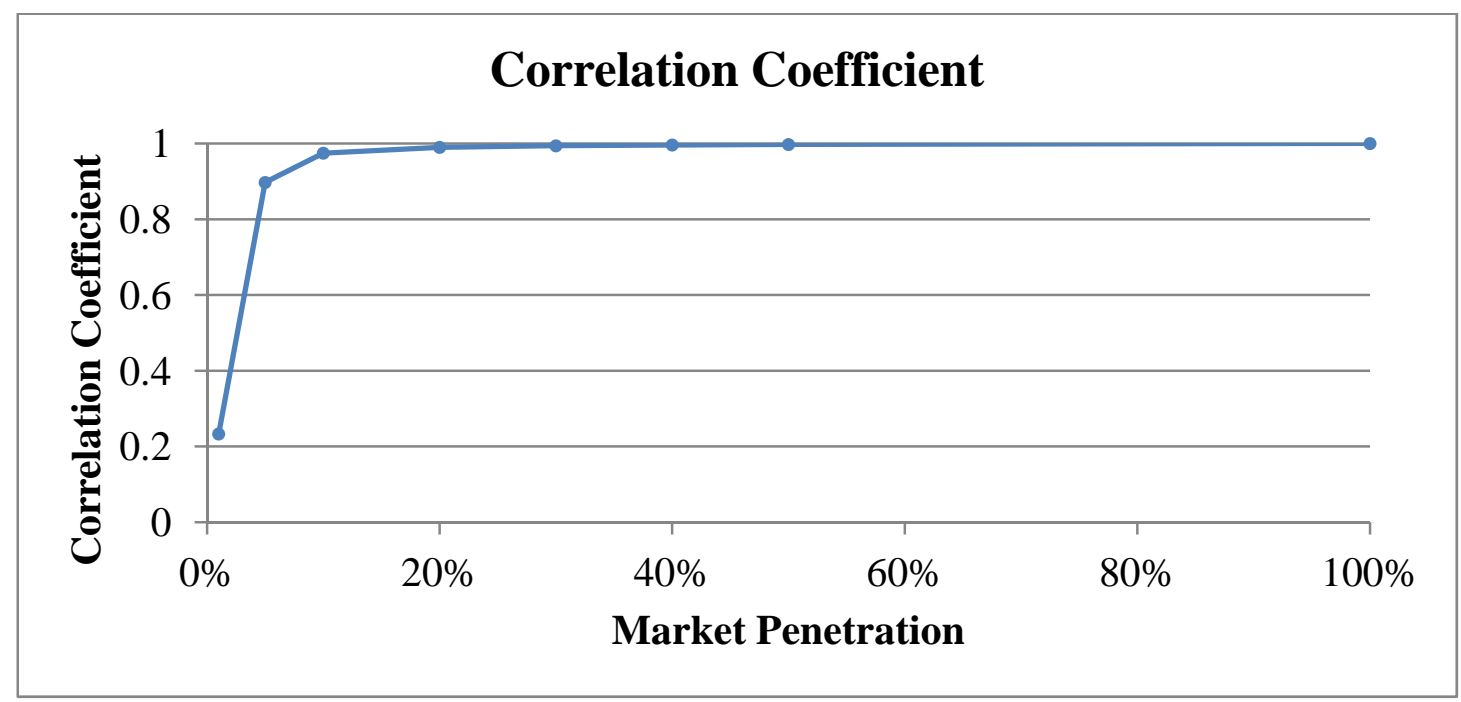

Figure 4-4: Correlation coefficient of speed estimates based on collected data

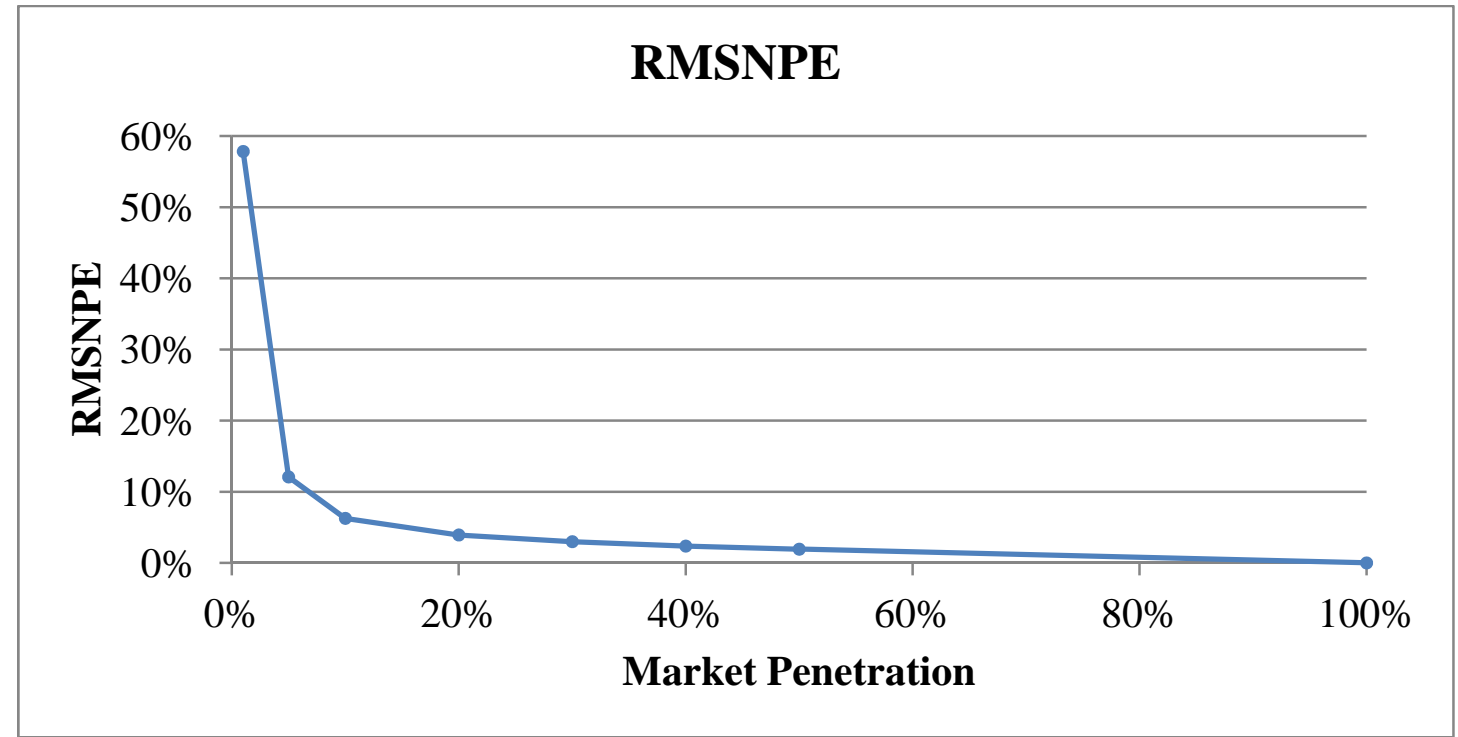

Figure 4-5: RMSNPE of speed estimates based on collected data

Figures 4-4 and 4-5 indicate that correlation coefficient between market penetration between the $20 \%$ rate and the $100 \%$ rate is 0.974 . The root mean squared 
normalized percent error of $20 \%$ market penetration is $3.93 \%$. It shows that having trajectory data of a sample size of at least $20 \%$ of drivers can be an accurate and reliable source of traffic data.

\subsection{Summary}

In this chapter, the field data acquisition efforts are discussed. First, a brief introduction to the study area is provided. Representative days are then identified by filtering out incident days, special events, and weekends. Using field data at representative days, the breakdown characteristics at bottlenecks are calculated. Finally, connected vehicle data collection and associated minimum market penetration to obtain reliable data are discussed. 


\section{CHAPTER 5}

\section{RESULTS}

As stated in the methodology chapter, simulation modeling is used as a tool to evaluate the developed VSL strategies in this study. This chapter presents the findings from the evaluation. First, this chapter presents the results from the proposed model calibration procedure that considers breakdown characteristics, capacity, traffic volume,

and system performance. This chapter then presents the results of the assessment of the impacts of various VSL strategies with different evaluated scenarios.

\subsection{Calibration}

The study area was modeled in the CORSIM micro-simulation model, as shown in Figure 5-1. This network had already been simulated in CORSIM by the FDOT during the process of the HOT lanes design. Elefteriadou et al. (2012) modified the configuration of the network and calibrated it to consider post-implementation conditions. In this study, calibration of the initial CORSIM files provided by Elefteriadou et al. (2012) is required to ensure that the simulation accurately reflects real-world conditions with consideration of breakdown characteristics.

Using contour speed maps and the breakdown information for different normal days presented in Section 4.2.1., May 12, 2010 was used as the day for the calibration of the basic parameters. The selected day has a median congestion level and bottlenecks that do not have a considerable effect on each other compared to other days. The simulation model was calibrated based on the abovementioned day, while considering two other selected days to make sure that the calibrated model was able to capture traffic variations 
and was not over-fitted to one special input data. In addition, sensitivity analyses on some global parameters were performed to ensure the accommodation of different traffic demands on different days. The stochasticity of breakdown was identified utilizing real-world data from different normal days in a one-year study period. In addition, as mentioned before, in order reduce congestion caused by two merging on-ramps, a fuzzy logic-based ramp metering strategy was implemented, operating from 3:30 PM until 6:00 PM. A run time extension (RTE) code was developed by Elefteriadou et al. (2012) to simulate this ramp-metering strategy in CORSIM, and it was also used in this study to simulate ramp metering.

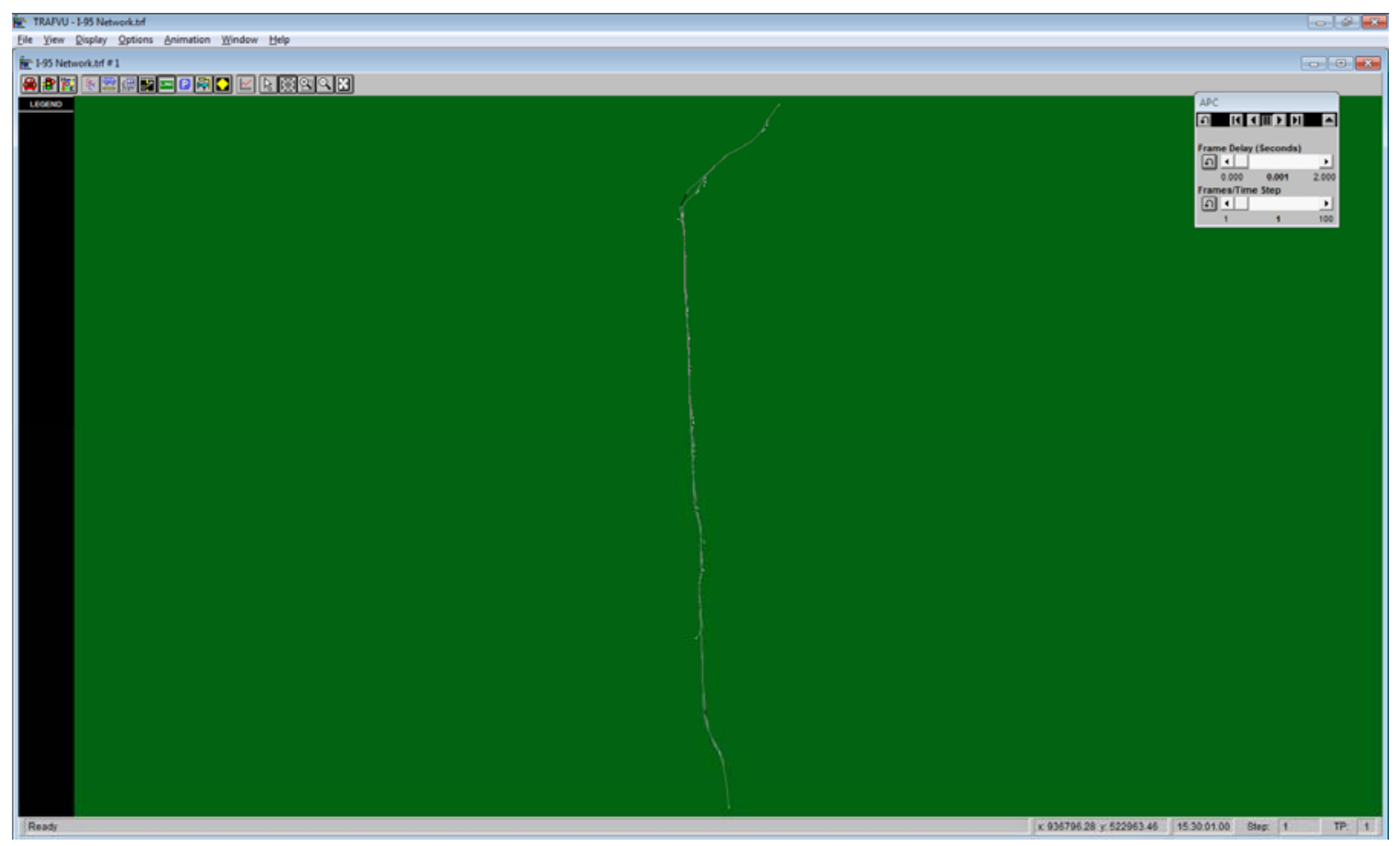

Figure 5-1: Screen shot of simulated network in CORSIM

The model was executed for 10 runs, each with different seed numbers. Table 5-1 presents the average network speed for each of the runs. Using Equation 3-4, based on an allowable error of $0.5 \%$ of the mean and a $95 \%$ confidence level, the required number of 
runs was estimated to be 9 . Hence, executing the model for 10 runs is deemed to be adequate.

Table 5-1: Average network speed for each simulation run

\begin{tabular}{ccccccccccc}
\hline Run \# & 1 & 2 & 3 & 4 & 5 & 6 & 7 & 8 & 9 & 10 \\
\hline Average & & & & & & & & & & \\
$\begin{array}{c}\text { Network } \\
\text { Speed (mph) }\end{array}$ & 48.22 & 47.89 & 48.48 & 47.46 & 48.43 & 48.35 & 47.81 & 48.42 & 48.69 & 48.20 \\
\hline
\end{tabular}

As described in the methodology section, these parameters were modified, taking into consideration characteristics such as capacity, traffic flows, flow-occupancy fundamental diagram, breakdown characteristics, travel times, and extents of queues. Tables 5-2 to 5-4 present the breakdown characteristics at the three bottlenecks based on the calibrated network results. In addition to presenting the results for each run, the results based on the average traffic data are also presented. Furthermore, the average and standard deviation of each measurement are presented, based on outputs of the 10 runs.

As the results indicate, the breakdown characteristics estimated based on the average results are smoothed compared to the results from individual runs, and the average conditions are less congested than most of the runs. This confirms that each run should be considered individually, in addition to averaging the results. 
Table 5-2: Breakdown characteristics at first bottleneck based on simulation result

\begin{tabular}{|c|c|c|c|c|c|c|c|c|}
\hline Run \# & $\begin{array}{l}\text { Starting } \\
\text { Time } \\
\text { (hh:mm) }\end{array}$ & $\begin{array}{l}\text { Duration } \\
\text { (hh:mm) }\end{array}$ & $\begin{array}{c}\text { Speed } \\
\text { Before } \\
\text { Breakdow } \\
n \\
(\mathrm{mph}) \\
\end{array}$ & $\begin{array}{l}\text { Pre-Brea } \\
\text { kdown } \\
\text { Flow } \\
\text { (veh/hr) }\end{array}$ & $\begin{array}{c}\text { Breakdow } \\
\text { n Speed } \\
\text { (mph) }\end{array}$ & $\begin{array}{c}\text { Queue } \\
\text { Discharg } \\
\mathrm{e} \\
\text { (veh/hr) }\end{array}$ & $\begin{array}{c}\text { Speed After } \\
\text { Breakdown } \\
\quad(\mathrm{mph})\end{array}$ & $\begin{array}{c}\text { Recovery } \\
\text { Flow After } \\
\text { Breakdown } \\
\text { (veh/hr) }\end{array}$ \\
\hline 1 & $15: 55$ & $0: 30$ & 43.83 & 7476 & 33.23 & 7358 & 47.30 & 7128 \\
\hline 2 & $16: 00$ & $1: 20$ & 48.51 & 7344 & 34.67 & 7306.4 & 49.88 & 6756 \\
\hline 3 & - & - & - & - & - & - & - & - \\
\hline 4 & $15: 45$ & $0: 35$ & 46.77 & 7392 & 31.33 & 7320 & 44.79 & 6864 \\
\hline 5 & $15: 30$ & $2: 05$ & 50.77 & 7428 & 32.68 & 7302 & 49.53 & 6888 \\
\hline 6 & $15: 30$ & $0: 50$ & 48.31 & 7488 & 32.65 & 7296 & 44.58 & 7344 \\
\hline 7 & $16: 25$ & $1: 00$ & 48.64 & 7428 & 34.21 & 7340 & 49.27 & 7032 \\
\hline 8 & $15: 55$ & $0: 35$ & 47.87 & 7572 & 32.64 & 7248 & 47.96 & 7056 \\
\hline 9 & - & - & - & - & - & - & - & - \\
\hline 10 & - & - & - & - & - & - & - & - \\
\hline $\begin{array}{l}\text { Average } \\
\text { traffic data }\end{array}$ & $16: 00$ & $1: 00$ & 44.67 & 7314 & 38.16 & 7252.2 & 44.69 & 7108.8 \\
\hline Average & $15: 51$ & $0: 59$ & 47.81 & 7447 & 33.06 & 7310 & 47.61 & 7010 \\
\hline $\begin{array}{l}\text { Standard } \\
\text { Deviation }\end{array}$ & $0: 17$ & $0: 31$ & 1.97 & 68 & 1.03 & 33 & 2.04 & 181 \\
\hline
\end{tabular}

Table 5-3: Breakdown characteristics at second bottleneck based on simulation result

\begin{tabular}{|c|c|c|c|c|c|c|c|c|}
\hline Run \# & $\begin{array}{l}\text { Starting } \\
\text { Time } \\
\text { (hh:mm) }\end{array}$ & $\begin{array}{l}\text { Duration } \\
\text { (hh:mm) }\end{array}$ & $\begin{array}{c}\text { Speed } \\
\text { Before } \\
\text { Breakdown } \\
(\mathrm{mph})\end{array}$ & $\begin{array}{l}\text { Pre-Brea } \\
\text { kdown } \\
\text { Flow } \\
\text { (veh/hr) }\end{array}$ & $\begin{array}{c}\text { Breakdow } \\
\text { n Speed } \\
\text { (mph) }\end{array}$ & $\begin{array}{c}\text { Queue } \\
\text { Discharg } \\
\text { e } \\
\text { (veh/hr) }\end{array}$ & $\begin{array}{c}\text { Speed After } \\
\text { Breakdown } \\
\text { (mph) }\end{array}$ & $\begin{array}{c}\text { Recovery } \\
\text { Flow After } \\
\text { Breakdown } \\
\text { (veh/hr) }\end{array}$ \\
\hline 1 & - & $2: 10$ & - & - & 36.62 & 7233 & 47.15 & 6912 \\
\hline 2 & $15: 25$ & $1: 45$ & 51.47 & 7248 & 36.84 & 7229 & 48.01 & 7212 \\
\hline 3 & $15: 25$ & $1: 50$ & 46.55 & 7164 & 36.69 & 7201 & 46.11 & 6984 \\
\hline 4 & $15: 25$ & $2: 10$ & 52.56 & 7332 & 37.48 & 7184 & 47.78 & 6684 \\
\hline 5 & $15: 30$ & $2: 20$ & 46.75 & 7236 & 37.83 & 7174 & 48.39 & 6876 \\
\hline 6 & $15: 40$ & $1: 30$ & 47.23 & 7320 & 36.74 & 7282 & 50.15 & 7032 \\
\hline 7 & $15: 35$ & $1: 55$ & 49.98 & 7368 & 38.15 & 7251 & 51.20 & 6636 \\
\hline 8 & $15: 30$ & $1: 50$ & 47.28 & 7248 & 37.69 & 7219 & 48.91 & 7152 \\
\hline 9 & $15: 25$ & $2: 10$ & 48.38 & 7224 & 36.50 & 7160 & 49.58 & 6984 \\
\hline 10 & $15: 15$ & $1: 00$ & 49.73 & 7296 & 33.93 & 7203 & 49.25 & 7080 \\
\hline $\begin{array}{l}\text { Average } \\
\text { traffic } \\
\text { data }\end{array}$ & $15: 30$ & $1: 15$ & 46.65 & 7237 & 38.79 & 7220 & 41.11 & 7172 \\
\hline Average & $15: 27$ & $1: 52$ & 48.88 & 7271 & 36.85 & 7214 & 48.65 & 6955 \\
\hline $\begin{array}{l}\text { Standard } \\
\text { Deviation }\end{array}$ & 0:06 & $0: 22$ & 2.05 & 60 & 1.12 & 35 & 1.41 & 176 \\
\hline
\end{tabular}


Table 5-4: Breakdown characteristics at third bottleneck based on simulation result

\begin{tabular}{lcccccccc}
\hline Run \# & $\begin{array}{c}\text { Starting } \\
\text { Thime } \\
\text { (hh:mm) }\end{array}$ & $\begin{array}{c}\text { Duration } \\
(\mathrm{hh}: \mathrm{mm})\end{array}$ & $\begin{array}{c}\text { Speed } \\
\text { Before } \\
\text { Breakdown } \\
\text { (mph) }\end{array}$ & $\begin{array}{c}\text { Pre-Brea } \\
\text { kdown } \\
\text { Flow } \\
\text { (veh/hr) }\end{array}$ & $\begin{array}{c}\text { Breakdow } \\
\text { n Speed } \\
(\mathrm{mph})\end{array}$ & $\begin{array}{c}\text { Queue } \\
\text { Discharg } \\
\text { e } \\
\text { (veh/hr) }\end{array}$ & $\begin{array}{c}\text { Speed After } \\
\text { Breakdown } \\
\text { (mph) }\end{array}$ & $\begin{array}{c}\text { Recovery Flow } \\
\text { After } \\
\text { Breakdown } \\
\text { (veh/hr) }\end{array}$ \\
\hline 1 & $17: 02$ & $0: 53$ & 50.1 & 7920 & 21.11 & 7758 & 51.35 & 6520 \\
2 & $16: 53$ & $0: 55$ & 51.17 & 8196 & 20.83 & 7894 & 52.39 & 7192 \\
3 & $17: 03$ & $0: 27$ & 52.04 & 8081 & 23.38 & 7986 & 51.53 & 7155 \\
4 & $16: 59$ & $1: 01$ & 48.58 & 8115 & 19.36 & 7505 & $-*$ & - \\
5 & $17: 05$ & $0: 55$ & 51.71 & 7950 & 19.6 & 7649 & - & - \\
6 & $17: 02$ & $0: 58$ & 50.06 & 7960 & 19.77 & 7614 & - & - \\
7 & $16: 56$ & $1: 04$ & 50.71 & 8200 & 19.01 & 7427 & - & - \\
8 & $16: 53$ & $0: 55$ & 51.11 & 8160 & 21.86 & 7857 & 50.67 & 7011 \\
9 & $16: 49$ & $1: 03$ & 52.2 & 8025 & 21.95 & 7816 & 51.96 & 7080 \\
10 & $17: 02$ & $0: 45$ & 51.43 & 7960 & 22.17 & 7773 & 52.63 & 6900 \\
$\begin{array}{l}\text { Average } \\
\text { traffic }\end{array}$ & $17: 02$ & $0: 50$ & 39.98 & 7819 & 22.64 & 7676 & 39.12 & 7214 \\
data & & & & & & & & \\
\hline $\begin{array}{l}\text { Average } \\
\text { Standard }\end{array}$ & $16: 58$ & $0: 53$ & 50.91 & 8057 & 20.90 & 7728 & 51.76 & 6976 \\
Deviation & $0: 05$ & $0: 09$ & 1.04 & 102 & 1.37 & 168 & 0.66 & 225 \\
\hline
\end{tabular}

*: The breakdown did not recover prior to 6:00 PM, ending time of this study's analysis.

The results presented in Tables 5-2 to 5-4 show that different simulation runs may be able to represent the stochastic nature of breakdown. For instance, at the first bottleneck, some of runs did not show traffic breakdown occurrence, as is the case with other runs, which were also observed in real-word conditions. In comparing breakdown speeds at different bottlenecks, it is noticed that the breakdown speed at the third bottleneck is significantly less than other bottlenecks. The standard deviation of breakdown speed at each bottleneck is about $1 \mathrm{mph}$. The starting time of breakdown occurrence varies between 17 minutes at the first bottleneck, to 5 minutes at the third bottleneck. Compared to the starting time of breakdowns, the variations of the durations of breakdowns at different bottlenecks are larger. Comparing pre-breakdown flow to queue discharge, the capacity drop after breakdown occurrence is between $1-4 \%$ at 
different bottlenecks. Speeds before breakdown and speeds after breakdown are considerably the same, which are similar to the free-flow speed.

Table 5-5 presents breakdown characteristics and comparisons of real-world data and simulation outcome. For this purpose, the breakdown characteristics of a specific date (May 12, 2010) and the average and standard deviation of different days are compared with the average and standard deviation of different runs. When comparing the breakdown characteristics in Tables $4-1$ to $4-3$ and 5-2 to 5-4, it should be noted that the variations in day-to-day real-world performance are due to the breakdown stochasticity and variations in traffic demands between days. The variation in the simulation seed numbers between runs only addresses the variations in the stochasticity of the breakdown. Further analysis is needed if demand variations are to be considered, in addition to varying the seed numbers to account for the capacity stochasticity. Another consideration in the calibration is that the stochasticity between runs can be influenced by changing the variances of different distributions in the model, including those related to vehicle and driver characteristics.

The results in Table 5-5 indicate that the main difference between the real-world and simulation outcomes is the capacity drop phenomenon, which can be calculated as the difference between the pre-breakdown flow and queue discharge rate. During this research, it was observed that although CORSIM microscopic simulation is capable of showing the drop in speed after breakdown, it fails to capture the capacity drop. This points out that the modeling of traffic behavior in CORSIM during breakdown conditions may need to be improved. In the interim, using the rubbernecking factor in CORSIM, which drops the capacity of the freeway at a specific time window, could be considered 
one solution to simulate the $5-10 \%$ drop in capacity due to breakdown that is observed in the real world.

TABLE 5-5: Breakdown characteristics comparison

\begin{tabular}{|c|c|c|c|c|c|c|c|c|c|c|}
\hline \multicolumn{3}{|c|}{ Bottleneck } & \multirow{2}{*}{$\begin{array}{c}\begin{array}{c}\text { Starting } \\
\text { Time } \\
\text { (hh:mm) }\end{array} \\
15: 35\end{array}$} & \multirow{2}{*}{$\begin{array}{c}\begin{array}{c}\text { Duration } \\
\text { (hh:mm) }\end{array} \\
1: 00\end{array}$} & \multirow{2}{*}{$\begin{array}{c}\text { Speed } \\
\text { Before } \\
\text { Breakd } \\
\text { own } \\
(\mathrm{mph})\end{array}$} & \multirow{2}{*}{$\begin{array}{c}\begin{array}{c}\text { Pre-Br } \\
\text { eakdo } \\
\text { wn } \\
\text { Flow } \\
\text { (veh/hr) }\end{array} \\
7672\end{array}$} & \multirow{2}{*}{ 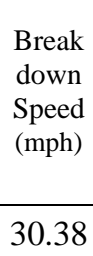 } & \multirow{2}{*}{$\begin{array}{c}\begin{array}{c}\text { Queue } \\
\text { Discharg } \\
\text { e } \\
\text { (veh/hr) }\end{array} \\
6794\end{array}$} & \multirow{2}{*}{$\begin{array}{c}\begin{array}{c}\text { Speed } \\
\text { After } \\
\text { Breakdo } \\
\text { wn } \\
(\mathrm{mph})\end{array} \\
56.95\end{array}$} & \multirow{2}{*}{$\begin{array}{c}\text { Recovery } \\
\text { Flow After } \\
\begin{array}{c}\text { Breakdown } \\
\text { (veh/hr) }\end{array} \\
6888\end{array}$} \\
\hline & & $5 / 12 / 10$ & & & & & & & & \\
\hline \multirow{4}{*}{$\# 1$} & \multirow{2}{*}{$\begin{array}{l}\text { Real- } \\
\text { World }\end{array}$} & Average & $15: 37$ & $2: 14$ & 55.67 & 7522 & 30.46 & 6721 & 54.36 & 6816 \\
\hline & & $\begin{array}{l}\text { Standard } \\
\text { Deviation }\end{array}$ & $0: 36$ & $0: 41$ & 2.01 & 143 & 1.50 & 105 & 3.01 & 85 \\
\hline & \multirow{2}{*}{$\begin{array}{c}\text { Simula } \\
\text { tion }\end{array}$} & Average & $15: 51$ & $0: 59$ & 47.81 & 7447 & 33.06 & 7310 & 47.62 & 7010 \\
\hline & & $\begin{array}{c}\text { Standard } \\
\text { Deviation }\end{array}$ & $0: 17$ & $0: 31$ & 1.97 & 68 & 1.03 & 33 & 2.03 & 181 \\
\hline \multirow{5}{*}{ \#2 } & \multirow{3}{*}{$\begin{array}{l}\text { Real- } \\
\text { World }\end{array}$} & $5 / 12 / 10$ & $15: 25$ & $2: 35$ & 59.48 & 7380 & 36.69 & 6840 & - & - \\
\hline & & Average & $15: 47$ & $2: 30$ & 56.49 & 7299 & 34.78 & 6727 & 50.29 & 6768 \\
\hline & & $\begin{array}{l}\text { Standard } \\
\text { Deviation }\end{array}$ & $0: 33$ & $0: 35$ & 3.80 & 152 & 1.53 & 95 & $0 *$ & 0 \\
\hline & \multirow{2}{*}{$\begin{array}{c}\text { Simula } \\
\text { tion }\end{array}$} & Average & $15: 27$ & $1: 52$ & 48.88 & 7271 & 36.85 & 7214 & 48.65 & 6955 \\
\hline & & $\begin{array}{l}\text { Standard } \\
\text { Deviation }\end{array}$ & 0:06 & $0: 22$ & 2.05 & 60 & 1.12 & 35 & 1.41 & 176 \\
\hline \multirow{5}{*}{ \#3 } & \multirow{3}{*}{$\begin{array}{l}\text { Real- } \\
\text { World }\end{array}$} & $5 / 12 / 10$ & $17: 00$ & $1: 00$ & 55.43 & 9192 & 23.03 & 7596 & - & - \\
\hline & & Average & $16: 32$ & $1: 27$ & 55.67 & 8880 & 24.60 & 7773 & 56.39 & 7632 \\
\hline & & $\begin{array}{l}\text { Standard } \\
\text { Deviation }\end{array}$ & $0: 17$ & $0: 17$ & 2.01 & 251 & 1.33 & 108 & $0 *$ & 0 \\
\hline & \multirow{2}{*}{$\begin{array}{c}\text { Simula } \\
\text { tion }\end{array}$} & Average & $16: 58$ & $0: 53$ & 50.91 & 8057 & 20.90 & 7728 & 51.76 & 6976 \\
\hline & & $\begin{array}{c}\text { Standard } \\
\text { Deviation }\end{array}$ & $0: 05$ & 0:09 & 1.04 & 102 & 1.37 & 168 & 0.66 & 225 \\
\hline
\end{tabular}

*: Only in one day, the breakdown recovered prior to 6:00 PM, ending time of this study's analysis.

In addition to pre-breakdown flow and queue discharge, the fundamental diagram is considered in the calibration process. Fundamental diagrams are constructed from both simulated and real-world data to present the flow-occupancy relationship at the bottleneck. In addition to capacity and capacity stochasticity, the diagram also shows the critical density, at which the flow reaches capacity. Figure 5-2 compares the 
occupancy-flow relationships for the three bottlenecks based on real-world and simulated data.

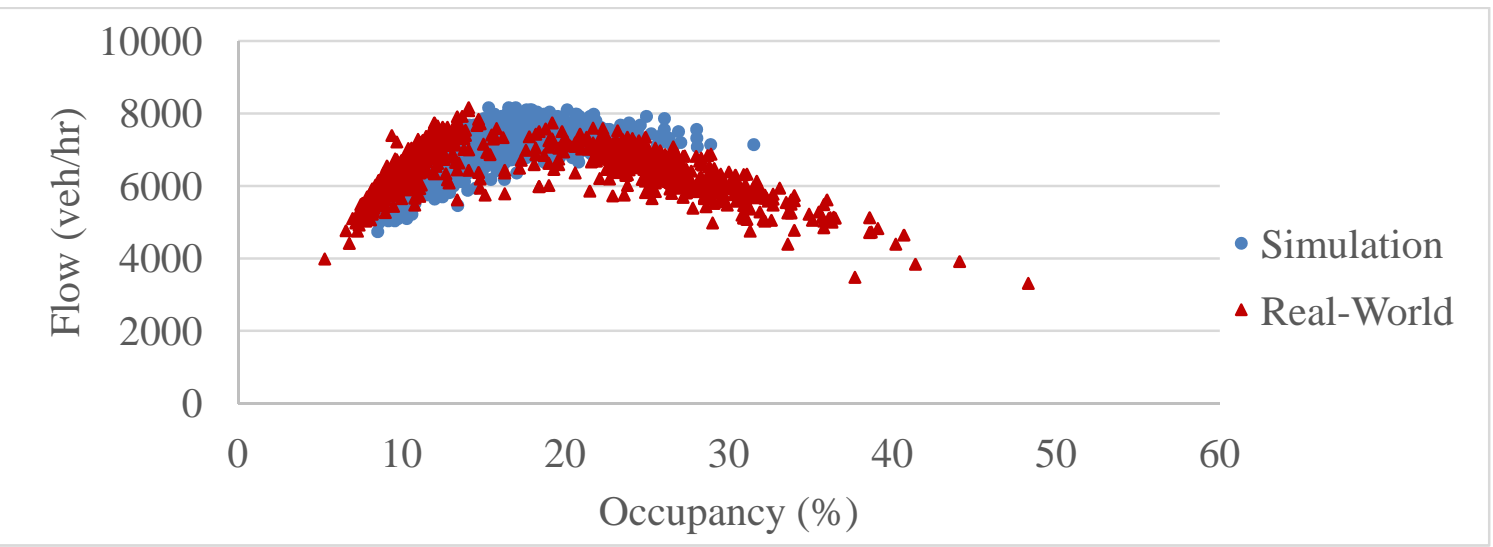

(a) Comparison of flow-occupancy relationship at first bottleneck

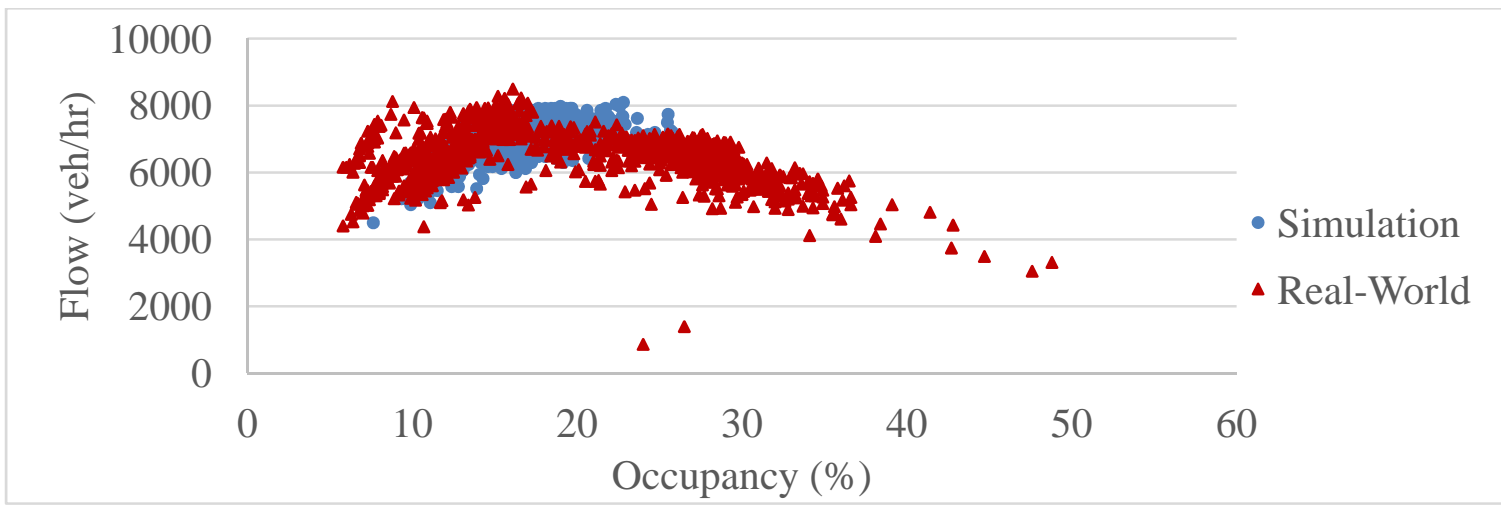

(b) Comparison of flow-occupancy relationship at second bottleneck

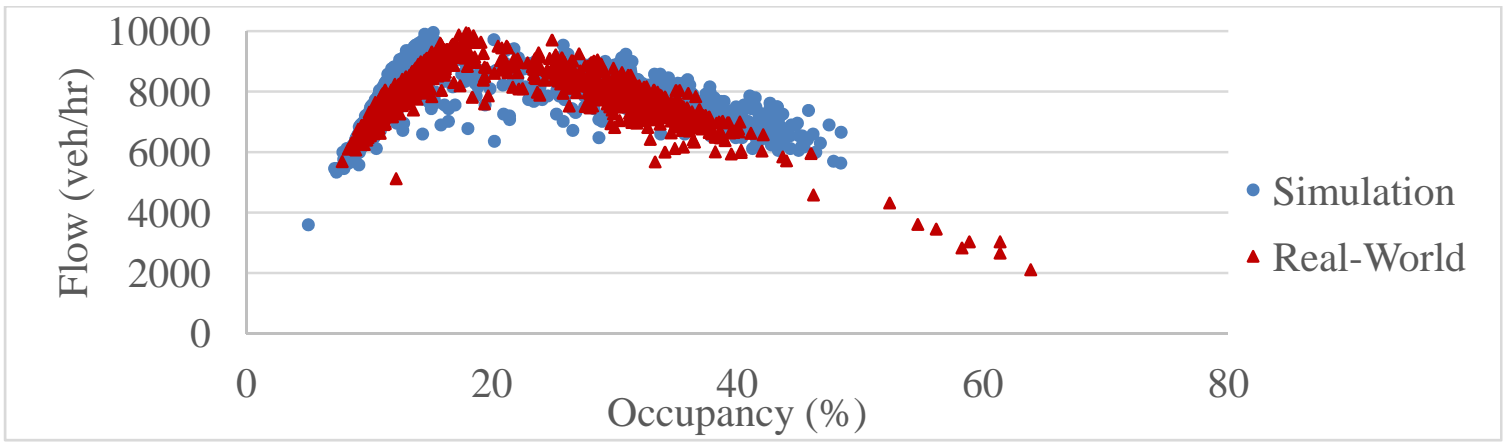

(c) Comparison of flow-occupancy relationship at third bottleneck

Figure 5-2: Comparison of flow-occupancy relationship between real-world and simulation 
As Figure 5-2 shows, the calibrated model is successful in replicating capacity and the related critical occupancy. It is interesting to note, however, that the traffic flow during congested conditions is a little higher in the simulation, compared to real-world conditions, possibly reflecting the drop in maximum throughput due to breakdown that was observed in the real-world, but not in the simulation.

Another part of the breakdown characteristics calibration step is to examine the capability of the simulation model to replicate breakdown probability. Breakdown probability models were developed based on detector measurements at three bottleneck locations from different normal days using Equation (3-5). These models were compared with the breakdown probability models based on simulation runs with different seed numbers. The comparisons are shown in Figure 5-3.

Figure 5-3 indicates that since the simulation results are based on only a 3-hour PM peak period, the range of data based on the real world is wider than the simulation data since a longer period is represented by the real-world model. However, it appears, particularly at the first and third bottleneck, that the simulation model thoroughly replicates the real-world breakdown probability. Even at the second bottleneck, the results of the real-world and simulation breakdown probabilities appear to be similar.

Table 5-6 presents the system performance measurements, mean absolute error, mean absolute normalized error, Theil's Inequality Coefficient, and root mean squared normalized percent error on volume and speed results of the calibrated model in different runs. In addition, the congestion index is calculated for each run to show how well the congestion network-wide is replicated. It should be noted that congestion index based on field data is 0.232 . This table also shows the correlation coefficient of volume. 


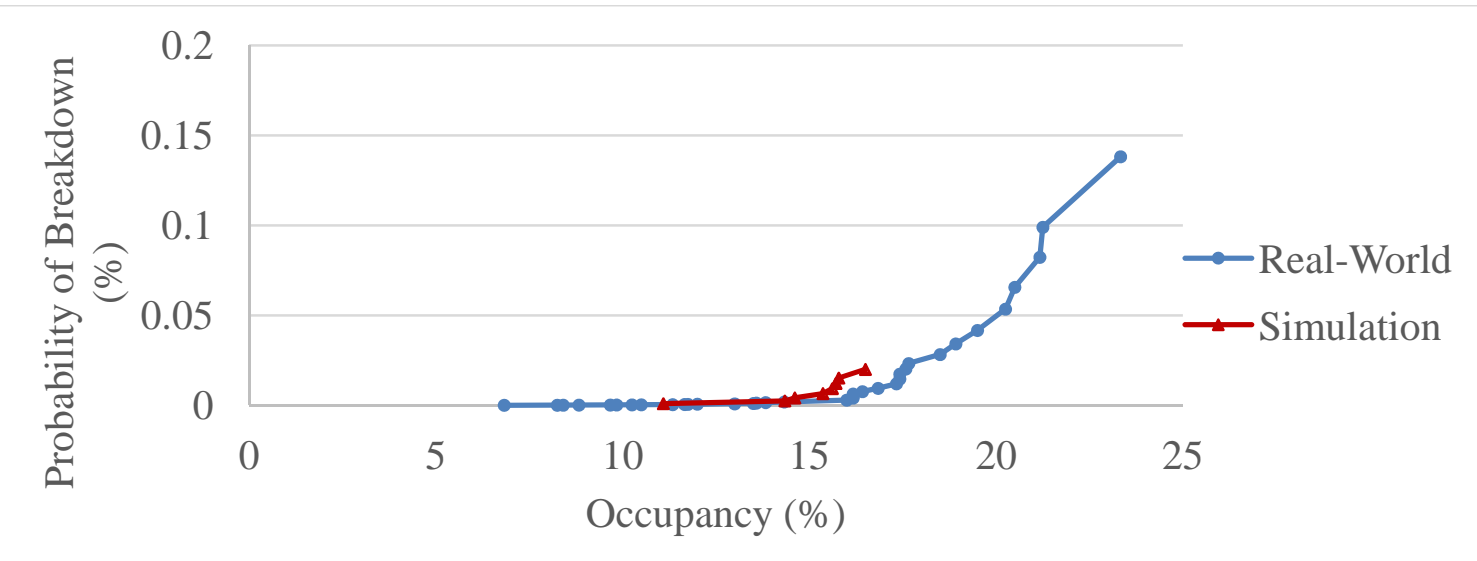

(a) Comparison of breakdown probability at first bottleneck

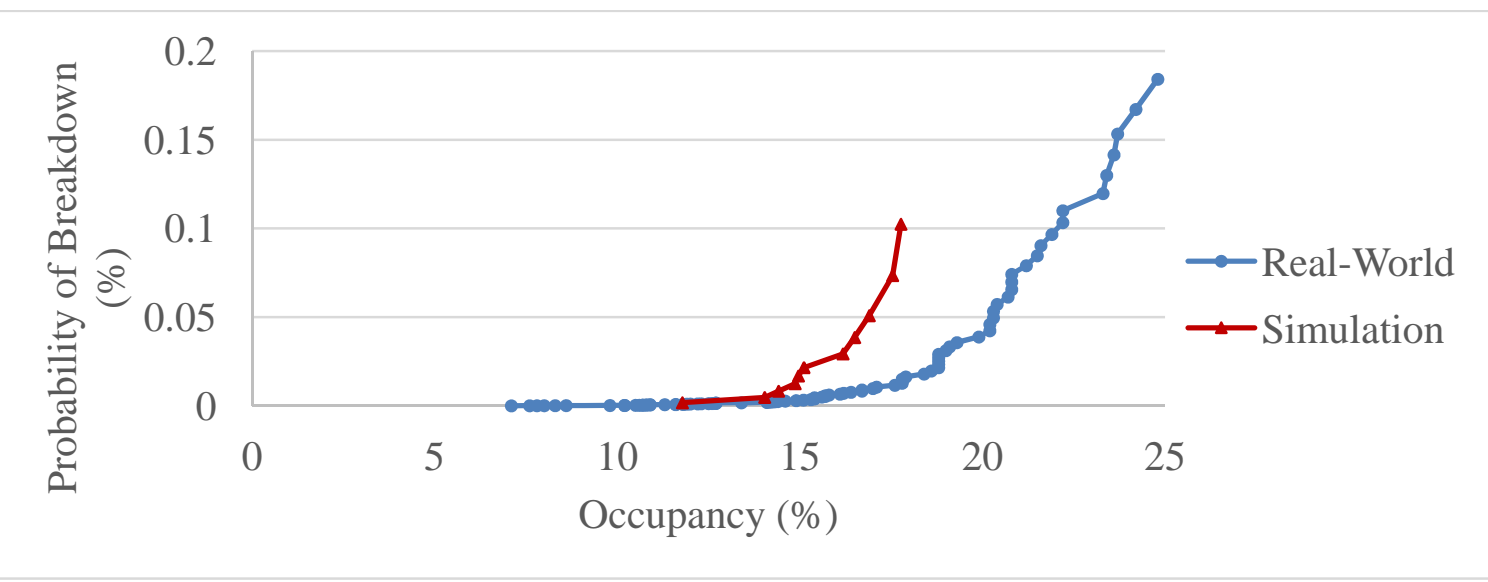

(b) Comparison of breakdown probability at second bottleneck

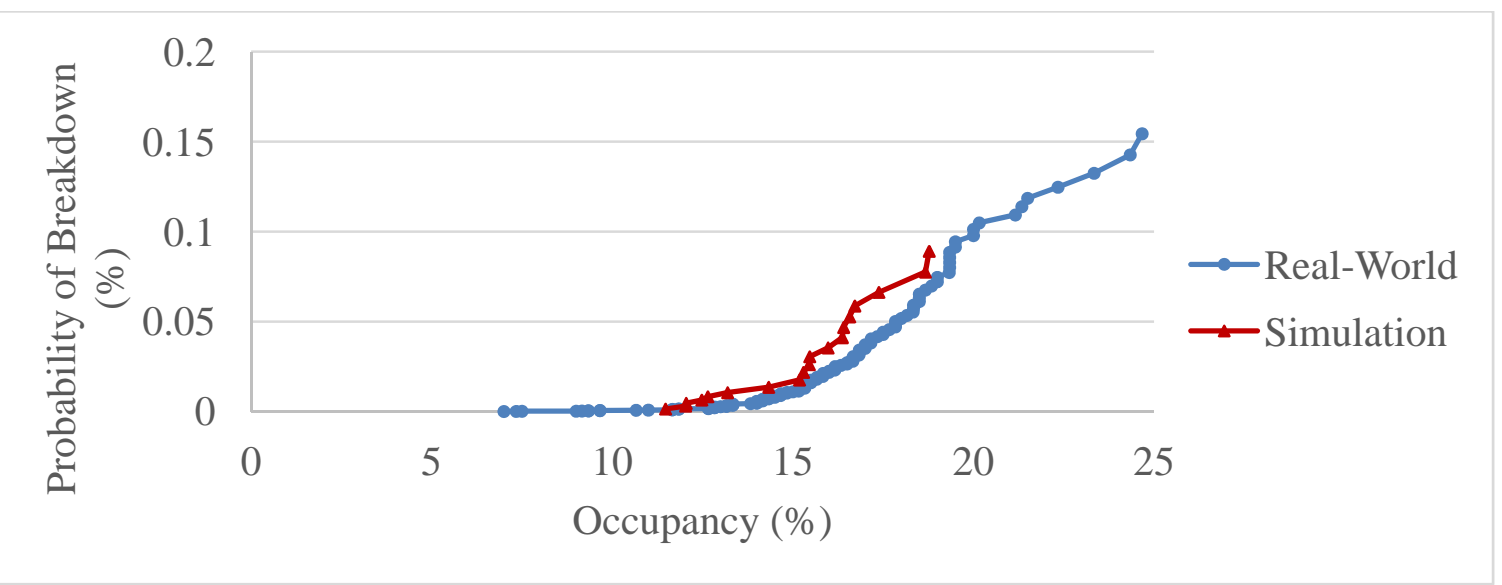

(c) Comparison of breakdown probability at third bottleneck

Figure 5-3: Comparison of breakdown probability between real-world and simulation

Visual validation is another tool that is used to validate the calibrated model. 
Figure 5-4 shows the speed contour map based on simulation results. A comparison between the speed contour map of simulated results and field data shows that the calibrated model adequately replicated the bottleneck location, shockwave, congestion in different locations of the network during different time intervals, and the speed pattern in general.

Table 5-6: Goodness-of-fit assessment of MOEs

\begin{tabular}{|c|c|c|c|c|c|c|c|c|c|c|}
\hline \multirow[t]{2}{*}{ Run \# } & \multirow{2}{*}{$\begin{array}{c}\text { Congest } \\
\text { ion } \\
\text { Index }\end{array}$} & \multicolumn{2}{|c|}{ MAE } & \multicolumn{2}{|c|}{ MANE } & \multicolumn{2}{|c|}{$\begin{array}{c}\text { Theil's Inequality } \\
\text { Coefficient }\end{array}$} & \multicolumn{2}{|c|}{ RMSNPE } & \multirow{2}{*}{$\begin{array}{c}\text { Correlation } \\
\text { Coefficient } \\
\text { Volume }\end{array}$} \\
\hline & & Speed & Volume & Speed & Volume & Speed & Volume & Speed & Volume & \\
\hline 1 & 0.212 & 5.90 & 368.04 & 0.12 & 0.06 & 0.09 & 0.04 & 31.57 & 8.62 & 0.94 \\
\hline 2 & 0.209 & 6.18 & 367.99 & 0.12 & 0.06 & 0.09 & 0.04 & 34.00 & 8.34 & 0.94 \\
\hline 3 & 0.195 & 6.23 & 376.76 & 0.13 & 0.06 & 0.10 & 0.04 & 36.19 & 8.74 & 0.93 \\
\hline 4 & 0.229 & 5.97 & 364.95 & 0.12 & 0.06 & 0.09 & 0.04 & 27.85 & 8.24 & 0.94 \\
\hline 5 & 0.246 & 6.07 & 354.81 & 0.12 & 0.06 & 0.10 & 0.04 & 27.82 & 8.18 & 0.94 \\
\hline 6 & 0.216 & 6.15 & 391.87 & 0.12 & 0.06 & 0.09 & 0.04 & 30.48 & 9.00 & 0.93 \\
\hline 7 & 0.232 & 6.11 & 378.85 & 0.12 & 0.06 & 0.09 & 0.04 & 28.74 & 8.79 & 0.94 \\
\hline 8 & 0.211 & 6.21 & 366.56 & 0.13 & 0.06 & 0.09 & 0.04 & 33.64 & 8.34 & 0.94 \\
\hline 9 & 0.224 & 6.62 & 359.64 & 0.13 & 0.06 & 0.10 & 0.04 & 34.03 & 8.32 & 0.94 \\
\hline 10 & 0.199 & 6.48 & 365.72 & 0.13 & 0.06 & 0.10 & 0.04 & 36.06 & 8.33 & 0.941 \\
\hline $\begin{array}{c}\text { Average } \\
\text { traffic } \\
\text { data }\end{array}$ & 0.217 & 5.90 & 353.68 & 0.12 & 0.06 & 0.09 & 0.04 & 29.51 & 8.16 & 0.94 \\
\hline
\end{tabular}

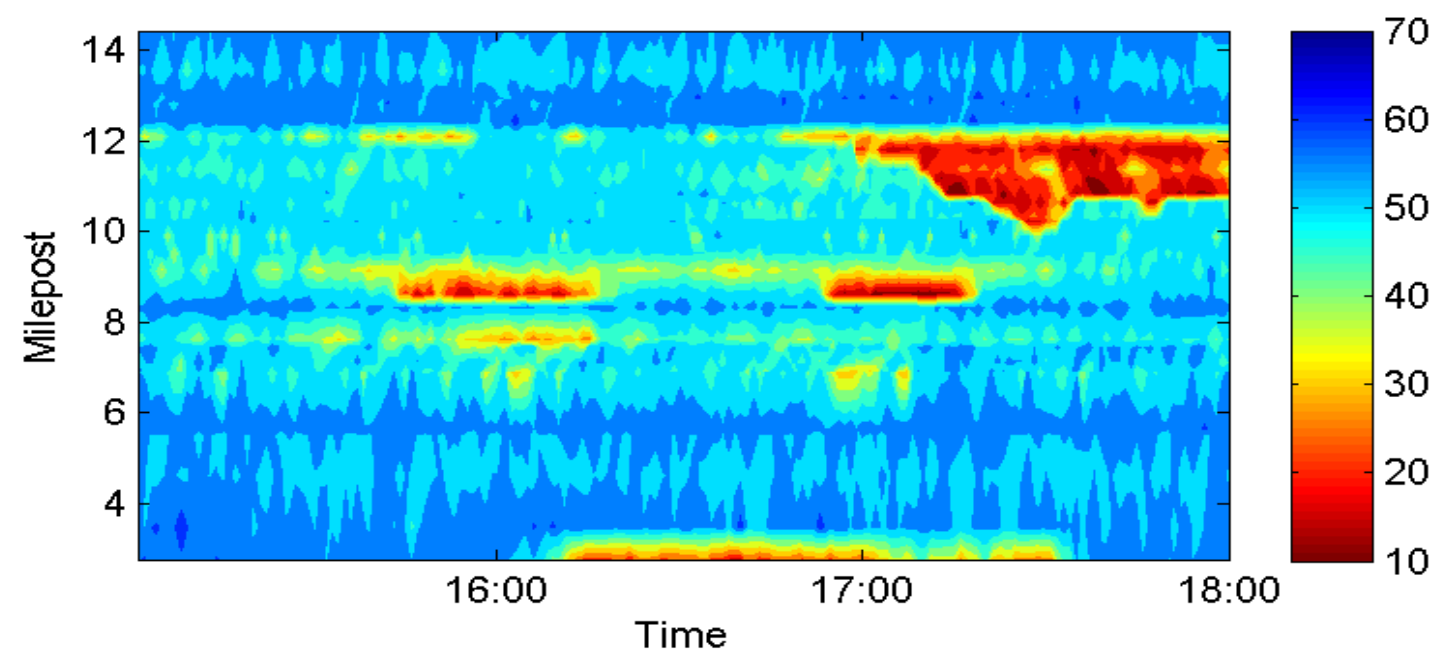

Figure 5-4: Speed contour map- simulation results 


\subsection{VSL Strategy Based on Infrastructure Detector Data}

The most severe bottleneck in the study area is the third bottleneck, as shown in Figure 5-5. This bottleneck is caused by spillback from an off-ramp. As congestion propagates, it reaches traffic volume entering from the managed lanes to the main lanes, which makes the speed at this location the lowest part of congested area. A lane-by-lane data analysis shows that the two left lanes that are affected by the spillback from the downstream off-ramp have significantly lower speeds and higher occupancies, compared to the three right lanes.

In this study, we only focused on investigating the effects of VSL alleviating the congestion caused by the third bottleneck. As field data have shown, this bottleneck is activated around 5:00 PM. This means that in the first two hours of the analysis, this bottleneck location is uncongested, and during the last hour, it is congested. This condition provides a unique opportunity to analyze VSL effects both before and after breakdown occurrence.

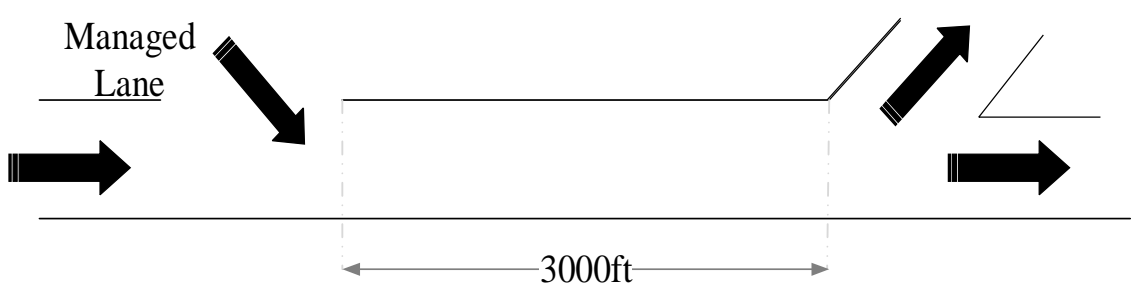

Figure 5-5: Third bottleneck scheme

The VSL system in this research was studied in two different aspects: 1) VSL effects on congestion, and 2) VSL effects on breakdown characteristics at the bottleneck. First, in order to highlight the effects of VSL on congestion, the area that could be affected by congestion when VSL is not implemented is found based on the real-world extent of the queue. Later, a congestion index is calculated as the mean relative difference 
of field speed against free-flow speed at all segments of this area using Equation 4-1. In addition, the maximum back of queue, identified based on the most upstream detector reached by congestion, is another measurement used to study the effects of VSL on congestion. The breakdown characteristics considered in this study are: the average speed during breakdown-breakdown speed (mph), starting time of breakdown (hh:mm), duration of breakdown (hh:mm), maximum pre-breakdown flow (veh/hr), and queue discharge rate (veh/hr).

Generally, the stochastic simulation models present different output values for repeated simulation runs with different seed numbers. CORSIM was run ten times with different random number seeds to account for the stochasticity in the results. In analyzing the VSL effects, the average of the values from the simulation runs with different seed numbers were used, as recommended by current practices and guidelines. However, when analyzing the VSL effects on breakdown characteristics, this study also considers each run individually and examines the breakdown characteristics from each individual run. This is due to different reasons in real-world simulations (under specific levels of demands): In one run, the breakdown may occur at a specific time, and in another run, it may occur during another time or it may not even occur at all. Using the average values of system performance would result in smoothed values that dilute the high congestion levels in runs with longer traffic breakdown durations. In addition, when examining the changes between time intervals in the average values of measures such as speed, sharp changes in these measures indicating breakdown would also be eliminated due to the aforementioned diluting effect. 
As explained earlier, this study identified the thresholds between different traffic regimes, of which the VSL is to be changed based on exhaustive searches. First, the various ranges for the potential thresholds were identified based on the fundamental diagram and probability of breakdown relationship, as shown in Figure 5-3 (c). Based on the identified range of occupancy for each traffic regime, 30 different combinations of thresholds were generated to perform the exhaustive search mentioned earlier. A comparison of the system's performance based on simulation results indicates that there are two combinations of thresholds that produce the best performance. Since one of these combinations was the same as the thresholds used in the VSL system implemented on the I-4 in Orlando, Florida (Elefteriadou 2012), this combination of thresholds was used in this research as the best combination, and is used in the remaining analysis of the paper. Table 5-7 presents the selected thresholds for different traffic regimes.

As stated in the previous section, the noise in the traffic detector data may lead to fluctuation in the posted speed limit, which has negative effects on the performance of the VSL system. Thus, data smoothing was used. Figure 5-6 shows the posted speed limit based on different strategies of data smoothing. The first three are based on a simple moving average method, and the last one is based on an exponential moving average method. Analyzing the results indicates that in terms of system performance, using the average of the last three minutes of data produces the best results, and it does not lead to fluctuation of the posted speed limit.

As mentioned earlier, one of the benefits of the VSL system is to change the critical occupancy to higher values. Figure 5-7 indicates that the calibrated CORSIM model was able to show this shift in critical occupancy, from $15 \%$ to $19.5 \%$. This shift in 
critical occupancy can have a positive impact on system performance by shifting the start of the unstable region of the diagram to a higher occupancy.

Table 5-7: Occupancy thresholds and sets of speed limits for traffic conditions

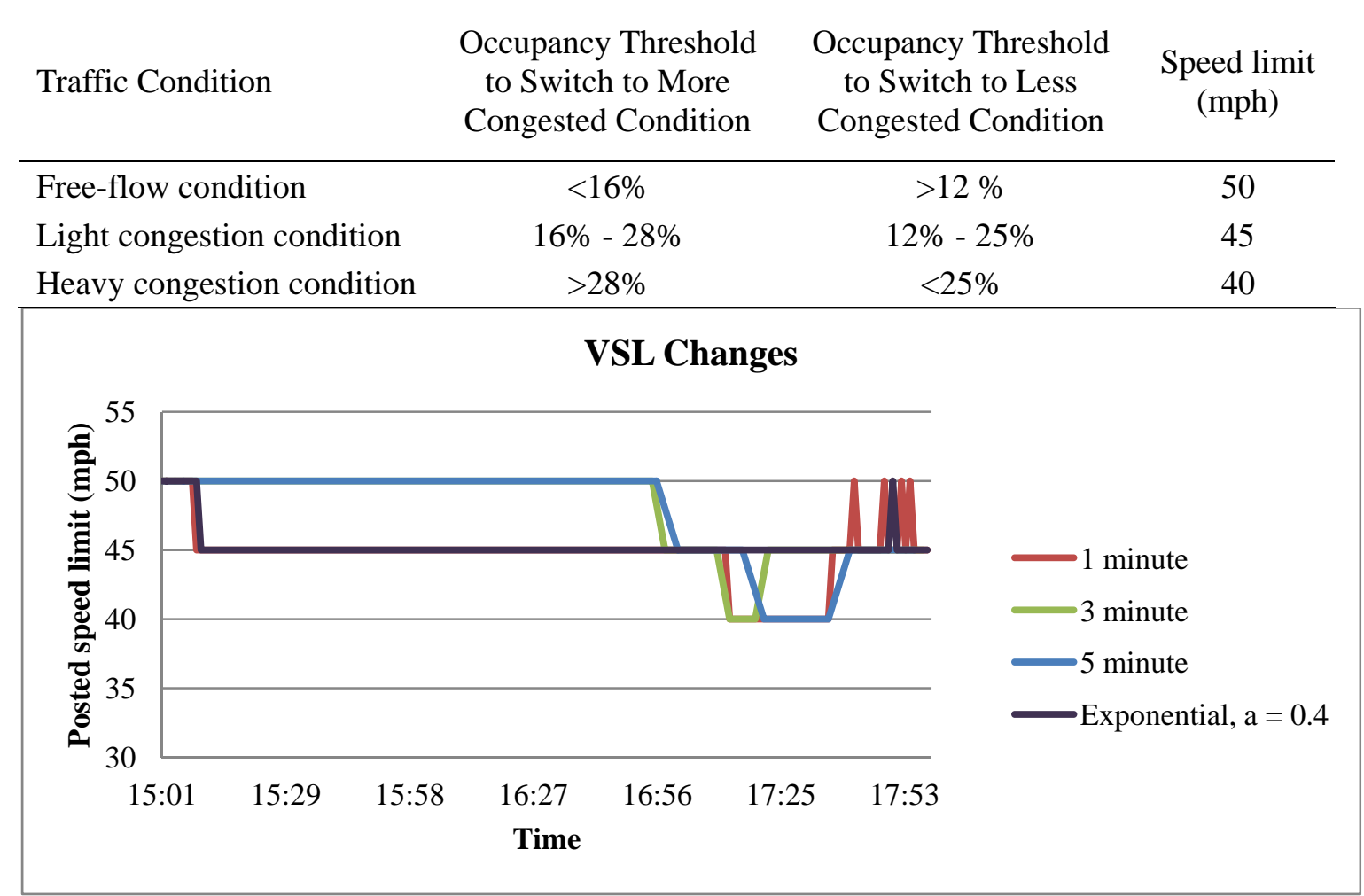

Figure 5-6: Posted speed limits based on different strategies of data smoothing

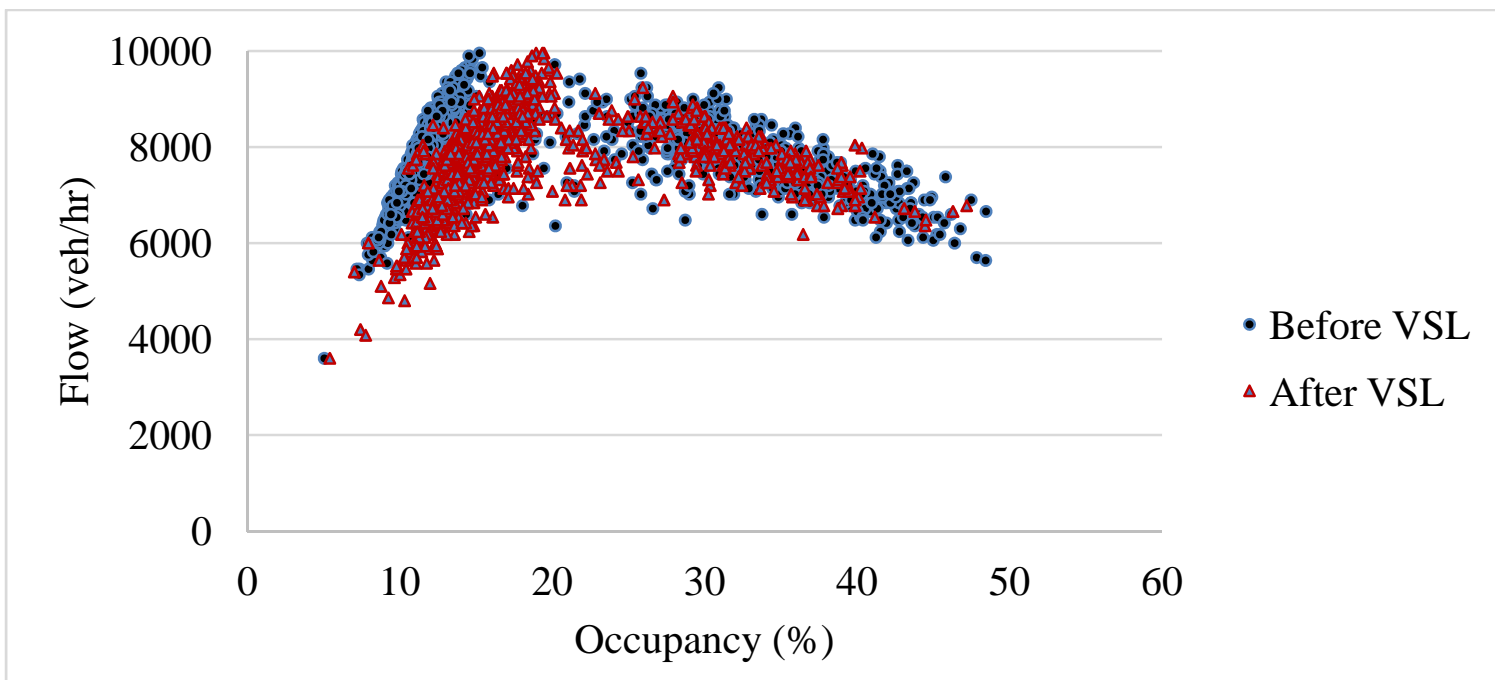

Figure 5-7: Comparison of flow-occupancy relationship between before and after VSL 
As explained earlier, in order to highlight the advantages of the proposed shockwave-based VSL, in which the VSL influence area is moved upstream and downstream with the changes in traffic conditions, this study compared the results from this approach with fixed-location VSLs. Four different fixed locations of the VSL signs upstream of the bottleneck were considered: 0.57 mile, 0.88 mile, 1.1 miles, and 1.32 miles. These alternative locations were selected, taking into consideration the geometric constraints of the freeway, such as ramp locations. The shockwave-based VSL locations are allowed to vary from a 0.57 -mile segment to 1.32 miles, depending on traffic conditions, as reflected by the probability of breakdown and the location of the back of the queue after breakdown. As previously mentioned, during the first two hours of the simulation analysis, the study's bottleneck is uncongested. The congestion index is calculated based only on data from the first two hours when there is no congestion, during the last hour when there is congestion, and for the entire study time period. Figure 5-8 shows the congestion index comparison for different scenarios for these different time periods.

As Figure 5-8 indicates that in all scenarios, deactivating the VSL at the head of congestion to encourage vehicles to accelerate produces the same or better results than not deactivating the VSL. Figure 5-8 (a) shows that among the investigated scenarios, the shockwave-based VSL offers better results than the fixed location-based VSLs. As the VSL influence area becomes larger during uncongested conditions, the travel time and thus the congestion index increase, which is shown in Figure 5-8 (b). During congestion, the shockwave-based VSL produces a better performance compared to the other scenarios, as shown in Figure 5-8 (c). Comparing fixed location-based VSLs during congestion 
indicates that there is an optimal VSL influence area.

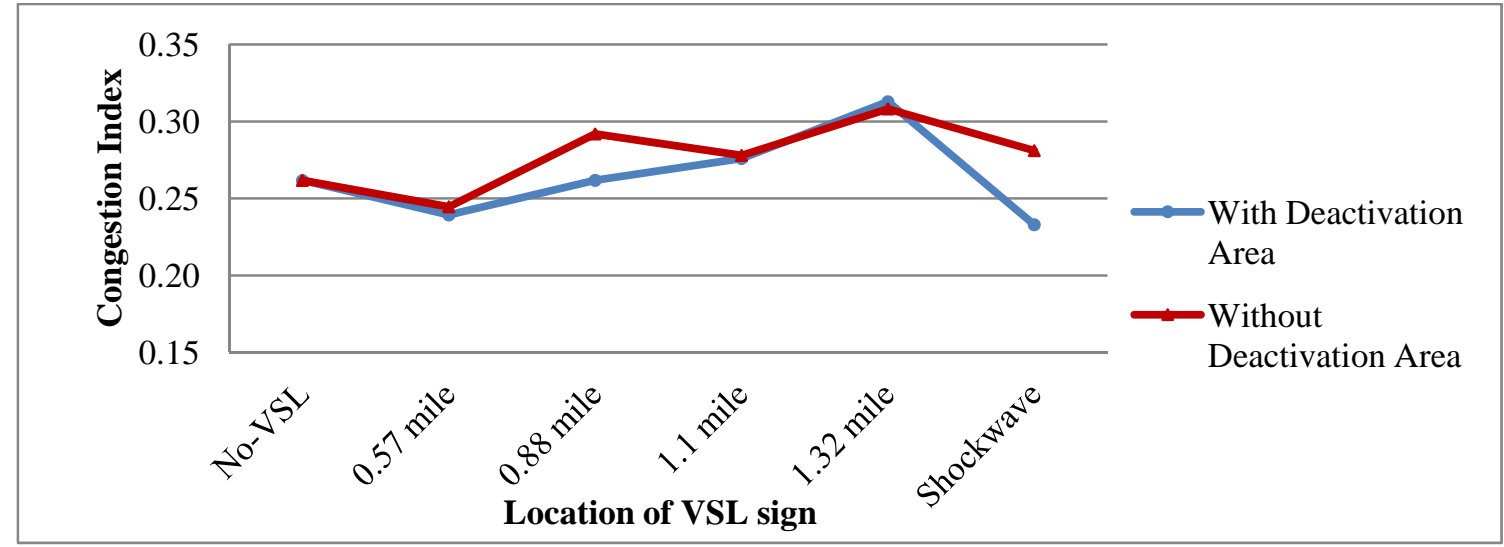

(a) Congestion index during entire study period

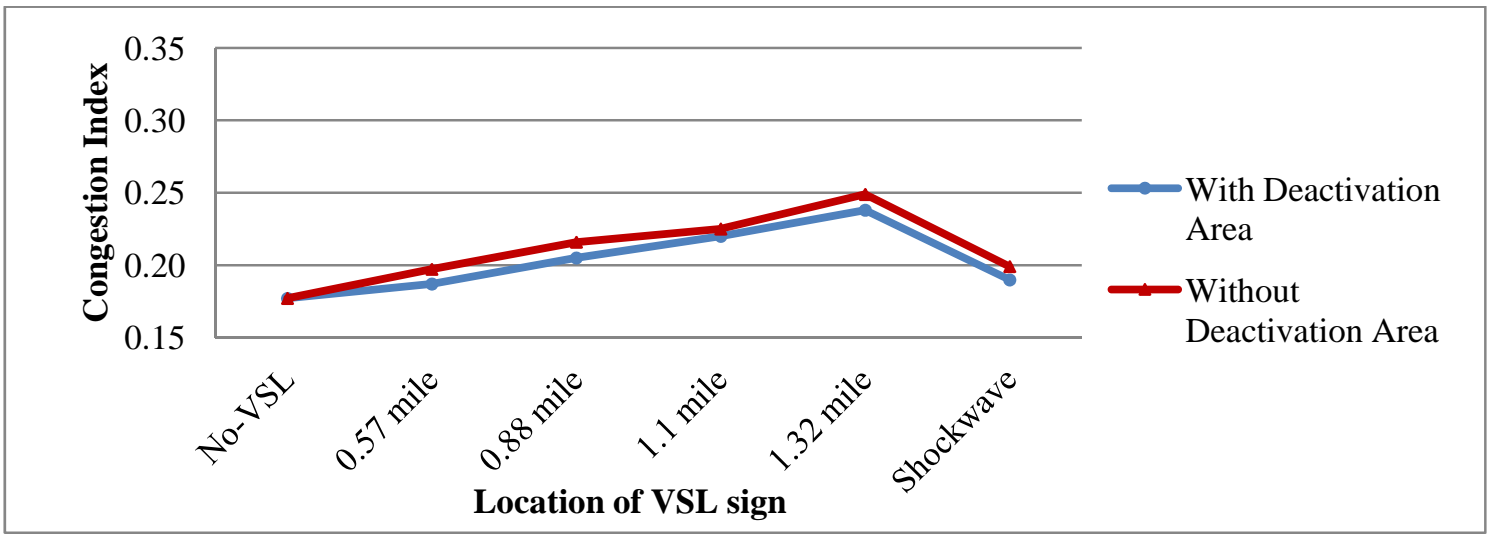

(b) Congestion index during uncongested conditions

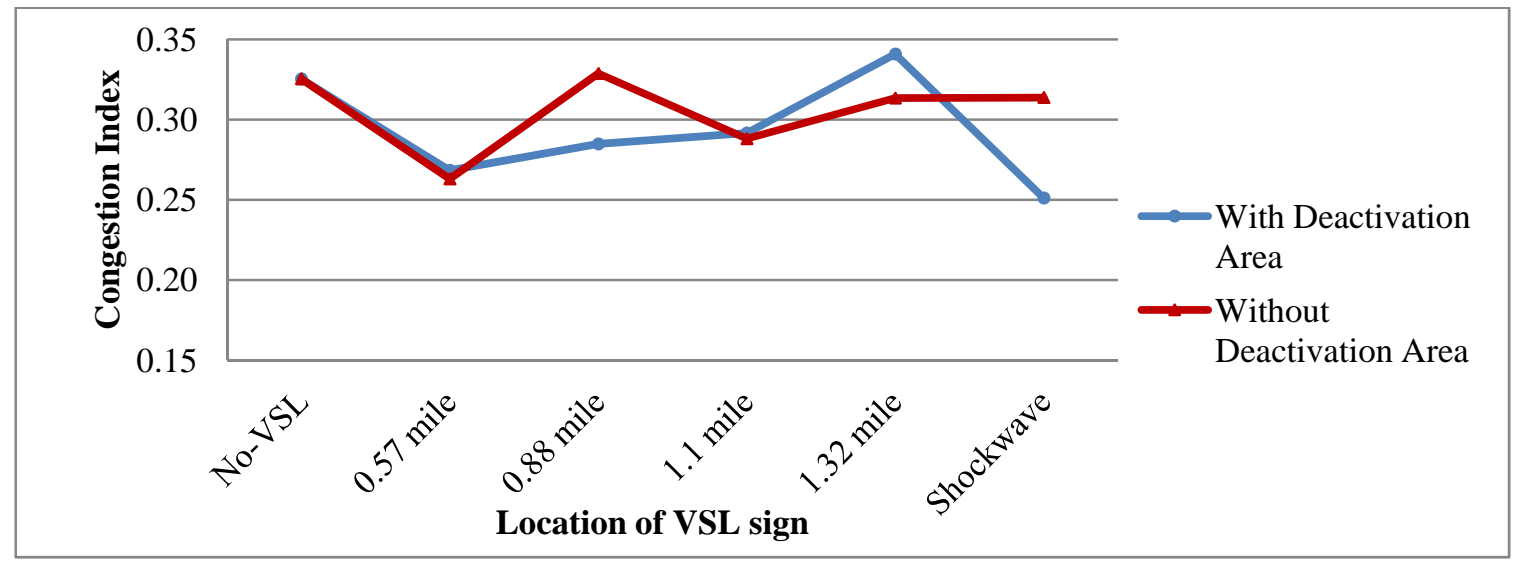

(c) Congestion index during congested conditions

Figure 5-8: Comparison of congestion index based on different VSL scenarios

In order to have a better understanding of VSL effects on congestion, Table 5-8 presents the congestion index and the maximum back of queue for each VSL alternative. 
The maximum back of queue was calculated using trajectory data from the simulation model. Table 5-9 displays the improvements in the congestion index and queue length when using each scenario. Table 5-9 shows that the increase of the VSL influence area produces a negative effect on traffic conditions during uncongested conditions. However, in general, the VSL in congested conditions improves congestion, but there is an optimal fixed VSL location, and the shockwave-based VSL performs better than the best fixed location VSL alternative. The maximum back of queue is reduced by 55\% when using the shockwave-based VSL system. In addition, the shockwave-based VSL improves the CI by $22 \%$, and by $10.5 \%$ for the congested and entire period, respectively.

Table 5-8: Congestion Index and maximum back of queue based different VSL systems

\begin{tabular}{lcccc}
\hline \multirow{2}{*}{ Scenario } & \multicolumn{3}{c}{ Congestion Index } & $\begin{array}{c}\text { Maximum Back of } \\
\text { Queue } \\
\text { (mile) }\end{array}$ \\
\cline { 2 - 4 } & $\begin{array}{c}\text { Entire Time } \\
\text { Period }\end{array}$ & $\begin{array}{c}\text { Uncongested } \\
\text { Condition }\end{array}$ & $\begin{array}{c}\text { Congested } \\
\text { Condition }\end{array}$ & 1.74 \\
Non-VSL & 0.262 & 0.177 & 0.325 & 1.14 \\
0.57 mile & 0.239 & 0.187 & 0.267 & 1.18 \\
1.1 mile & 0.262 & 0.205 & 0.285 & 1.05 \\
1.32 mile & 0.276 & 0.220 & 0.292 & 1.35 \\
shockwave-based & 0.313 & 0.238 & 0.341 & 0.78 \\
\hline
\end{tabular}

Table 5-9: Effects and improvements of each scenario on congestion

\begin{tabular}{lcccc}
\hline \multirow{2}{*}{ Scenario } & \multicolumn{3}{c}{ Congestion Index } \\
\cline { 2 - 4 } & $\begin{array}{c}\text { Entire Time } \\
\text { Period }\end{array}$ & $\begin{array}{c}\text { Uncongested } \\
\text { Condition }\end{array}$ & $\begin{array}{c}\text { Congested } \\
\text { Condition }\end{array}$ & $\begin{array}{c}\text { Maximum Back of } \\
\text { Queue }\end{array}$ \\
\hline 0.57 mile & $8.55 \%$ & $-5.64 \%$ & $17.52 \%$ & $34.48 \%$ \\
0.88 mile & $-0.03 \%$ & $-15.79 \%$ & $12.46 \%$ & $32.18 \%$ \\
1.1 miles & $-5.45 \%$ & $-24.22 \%$ & $10.37 \%$ & $39.66 \%$ \\
1.32 mile & $-19.55 \%$ & $-34.41 \%$ & $-4.76 \%$ & $22.41 \%$ \\
shockwave-based & $10.97 \%$ & $-7.22 \%$ & $22.82 \%$ & $55.17 \%$ \\
\hline
\end{tabular}


Table 5-10: Breakdown conditions at the simulated bottleneck under different scenarios

\begin{tabular}{lcccccc}
\hline Scenario & $\begin{array}{c}\text { Start } \\
\text { time } \\
\text { (hh:mm) }\end{array}$ & $\begin{array}{c}\text { Duration } \\
\text { (hh:mm) }\end{array}$ & $\begin{array}{c}\text { Speed } \\
\text { Before } \\
\text { Breakdown } \\
\text { (mph) }\end{array}$ & $\begin{array}{c}\text { Maximum } \\
\text { pre-Breakdown } \\
\text { Flow } \\
\text { (veh/hr) }\end{array}$ & $\begin{array}{c}\text { Breakdown } \\
\text { Speed } \\
\text { (mph) }\end{array}$ & $\begin{array}{c}\text { Queue } \\
\text { Discharge } \\
\text { (veh/hr) }\end{array}$ \\
\hline Non-VSL & $16: 52$ & $1: 13$ & 50.91 & 8057 & 20.9 & 7728 \\
0.57 mile & $16: 59$ & $0: 48$ & 40.05 & 8116 & 25.45 & 7733 \\
0.88 mile & $17: 02$ & $0: 50$ & 39.9 & 8062 & 25.06 & 7868 \\
1.1 mile & $17: 04$ & $0: 50$ & 39.81 & 8007 & 26.33 & 7833 \\
1.32 mile & $17: 03$ & $0: 48$ & 40.17 & 8102 & 25.48 & 7770 \\
$\begin{array}{l}\text { shockwave- } \\
\text { based }\end{array}$ & $17: 10$ & $0: 30$ & 38.98 & 8352 & 28.39 & 7935 \\
\hline
\end{tabular}

Table 5-10 presents a comparison of breakdown characteristics under different VSL alternatives to study the VSL's effect on breakdown conditions at the bottleneck. Table 5-10 indicates that all scenarios are successful in postponing traffic breakdown. The first observation is that all fixed location VSL scenarios have more or less the same effects on breakdown characteristics. The shockwave-based VSL has more positive impacts on breakdown than the fixed location VSLs. While the shockwave-based VSL postpones the breakdown for 18 minutes on average, the fixed location VSLs postpone it for 7 to 12 minutes, depending on the location of the VSL system. The main reason for the better performance of the shockwave-based VSL in postponing breakdown occurrence is that as the occupancy increases and the network becomes more congested based on occupancy measurements, which reflects a higher priority of breakdown, the VSL influence area starts to become extended upstream. The shockwave-based VSL significantly decreases the duration of the breakdown by 43 minutes, which is a $59 \%$ decrease in duration. The fixed VSL scenarios reduced the breakdown duration by 22 to 25 minutes, which is about a 35\% decrease. Unlike the non-VSL conditions where the pre-breakdown speed is about $50 \mathrm{mph}$, the pre-breakdown speed with VSL was $40 \mathrm{mph}$. 
Traffic speed during the breakdown was increased by $7.5 \mathrm{mph}$, which means a $36 \%$ improvement in traffic speed during the breakdown for the shockwave-based VSL system. The traffic speed during breakdown was increased from $22 \%$ to $26 \%$ with fixed location VSLs. The impact of VSL on the pre-breakdown capacity and queue discharge was small, according to the simulation analysis.

\subsection{VSL Strategy Based on Connected Vehicle Data}

As with the VSL strategy based on infrastructure detector data, this strategy aims to address the congestion at the third bottleneck. For this purpose, at each time step (1 second), the individual vehicle's data, speed, and location were collected from the simulation runs. After collecting speed data, in order to find the location of the congested area in the network, a wavelet transform was applied to speed data. Figure 5-9 presents the calculation of wavelet energy of speed data across the network.

Figure 5-9 (c) shows the temporal distribution of the energy. The lighter regions of the contour represent larger values of the wavelet transform coefficients, which indicate higher wavelet energy. Figure 5-9 (d) shows the wavelet energy for the speed location-series, which was computed using Equation (3-17), and indicates the head and tail of the congested area.

Tables 5-11 and 5-12 show the results of the VSL strategy based on the Connected Vehicle data and compared it to the VSL strategy based on Infrastructure detector data. Note that the infrastructure detector data-based strategy and Connected Vehicle data-based strategy used in this comparison are both shockwave-based VSLs. 

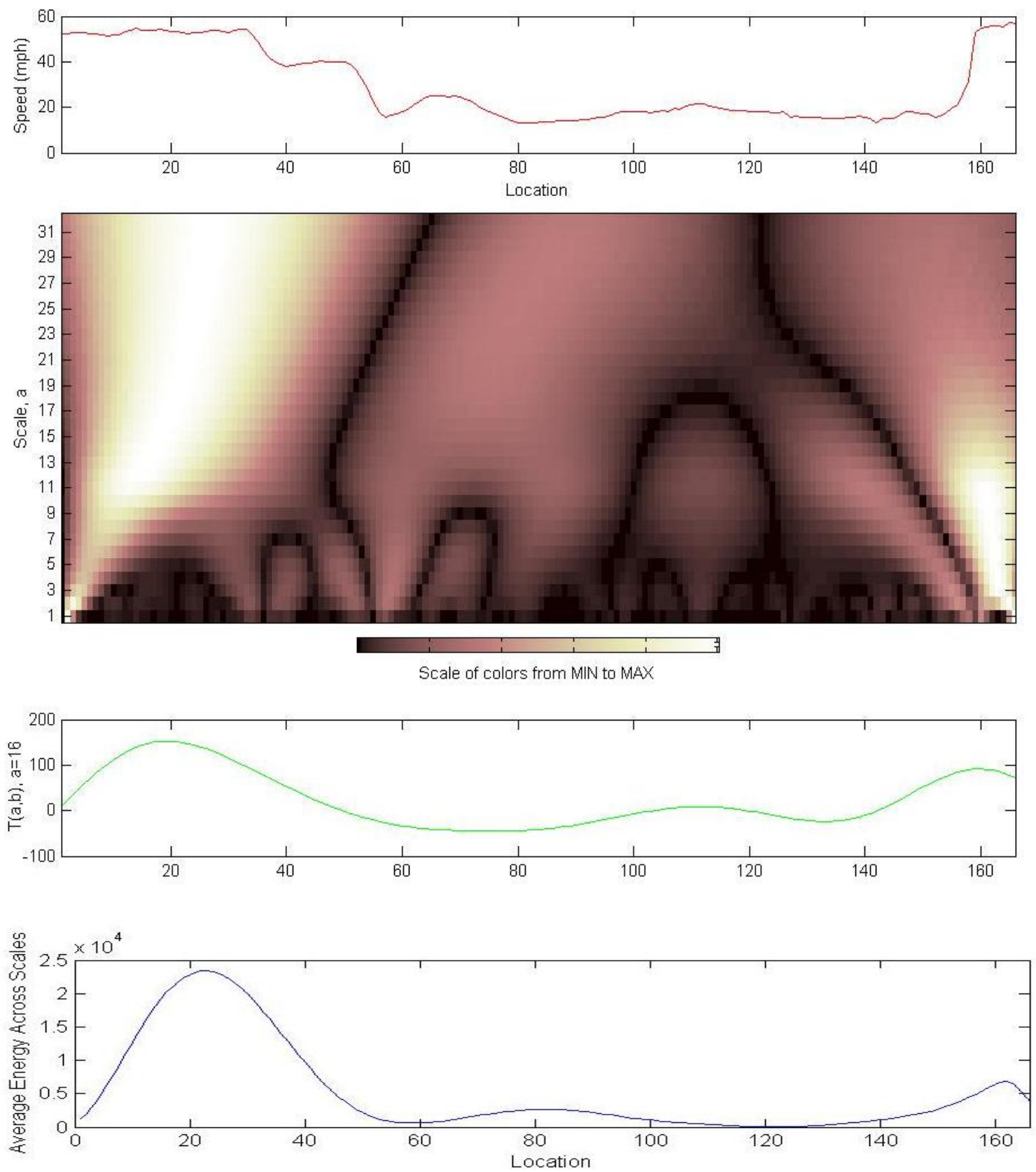

Figure 5-9: Illustration of wavelet transform and energy calculation. (a) Location series plot of speed at 17:25:00 pm; (b) Contour map of the absolute values of wavelet transform coefficients, $|T(\alpha, \beta)|$, from scale $\alpha=1-32$; (c) WT coefficients, $T(\alpha, \beta)$, at scale $\alpha=16$; (d) The temporal distribution of average wavelet-based energy across scales. 
Table 5-11: Congestion Index and maximum back of queue

\begin{tabular}{lcccc}
\hline \multirow{2}{*}{ Scenario } & \multicolumn{3}{c}{ Congestion Index } & Maximum \\
\cline { 2 - 4 } & $\begin{array}{c}\text { Entire time } \\
\text { period }\end{array}$ & Uncongested & Congested & $\begin{array}{c}\text { Back of } \\
\text { Queue (mile) }\end{array}$ \\
\hline Non-VSL & 0.313 & 0.238 & 0.341 & 1.32 \\
Infrastructure detector data based & 0.233 & 0.19 & 0.251 & 0.71 \\
Connected Vehicle data based & 0.234 & 0.19 & 0.252 & 0.71 \\
\hline
\end{tabular}

Table 5-12: Breakdown conditions at bottleneck

\begin{tabular}{lcccccc}
\hline \multicolumn{1}{c}{ Scenario } & $\begin{array}{c}\text { Start time } \\
\text { (hh:mm) }\end{array}$ & $\begin{array}{c}\text { Duration } \\
\text { (hh:mm) }\end{array}$ & $\begin{array}{c}\text { Speed Before } \\
\text { Breakdown } \\
\text { (mph) }\end{array}$ & $\begin{array}{c}\text { Maximum } \\
\text { Pre-Breakdown } \\
\text { Flow } \\
\text { (veh/hr) }\end{array}$ & $\begin{array}{c}\text { Breakdown } \\
\text { Speed } \\
\text { (mph) }\end{array}$ & $\begin{array}{c}\text { Queue } \\
\text { Discharge } \\
\text { (veh/hr) }\end{array}$ \\
\hline $\begin{array}{l}\text { Non-VSL } \\
\begin{array}{l}\text { Infrastructure } \\
\text { detector data } \\
\text { based }\end{array}\end{array}$ & $16: 52: 00$ & $1: 13$ & 50.91 & 8057 & 20.9 & 7728 \\
$\begin{array}{l}\text { Connected } \\
\text { Vehicle data } \\
\text { based }\end{array}$ & $17: 10: 00$ & $0: 30$ & 38.98 & 8352 & 28.39 & 7935 \\
\hline
\end{tabular}

As the results indicate, there are small differences between these two VSL strategy outcomes. The main reasons could be attributed to:

1. The locations of the traffic detectors are not far from each other; thus, the location of back of queue can be identified adequately with infrastructure detector data.

2. Although the Connected Vehicle technology allows more of collecting and detailed information of the current location of congestion, the speed limit cannot be changed as frequently as needed in space and time to take advantage of these detailed data due to safety and compliance concerns.

3. The tested methods do not fully utilize the new types of information gathered from Connected Vehicle. 


\subsection{Compliance Rate}

As mentioned earlier, one of the most important factors in having a successful and effective VSL system is the compliance rate. In this research, the VSL were implemented with different compliance rates and were estimated and compared. As stated earlier, two different ways of informing drivers about speed limits were tested: 1) by VSL sign, and 2) by Connected Vehicle through V2I communication. A difference between these two approaches is how to send the information to drivers in the segment. In the VSL sign approach, only drivers that are about to enter the segment will be notified about the posted speed limit, while drivers in the segment consider the speed limit that they saw while entering the segment as the speed limit, even if it changes. However, for the Connected Vehicle, the speed limit is sent to all drivers based on their location. Different compliance rates based on these two approaches were tested to find the lowest compliance rate that provides an effective VSL system. Figure 5-10 shows the congestion index for different compliance rates for different time windows using the shockwave-based VSL system.

As the results indicate, using the Connected Vehicle to inform drivers is better than using the VSL sign. During the uncongested period, as the compliance rate decreases, traffic speed increases, and as a result, the congestion index decreases slightly. During a congested period, having $40 \%$ of drivers comply with the posted speed limit results in higher congestion rate than a lower compliance rate. This result may indicate that having an equal number of compliant and incompliant drivers may result in disturbances with adverse effects on traffic. The results show that informing drivers through Connected Vehicle may stop this situation from happening; this issue needs to be further investigated. 
As expected, having $100 \%$ of compliance produces considerably better results than the lower compliance rates. Tables 5-13 and 5-14 indicate breakdown characteristics based on different compliance rates for both the VSL sign and Connected Vehicle respectively.

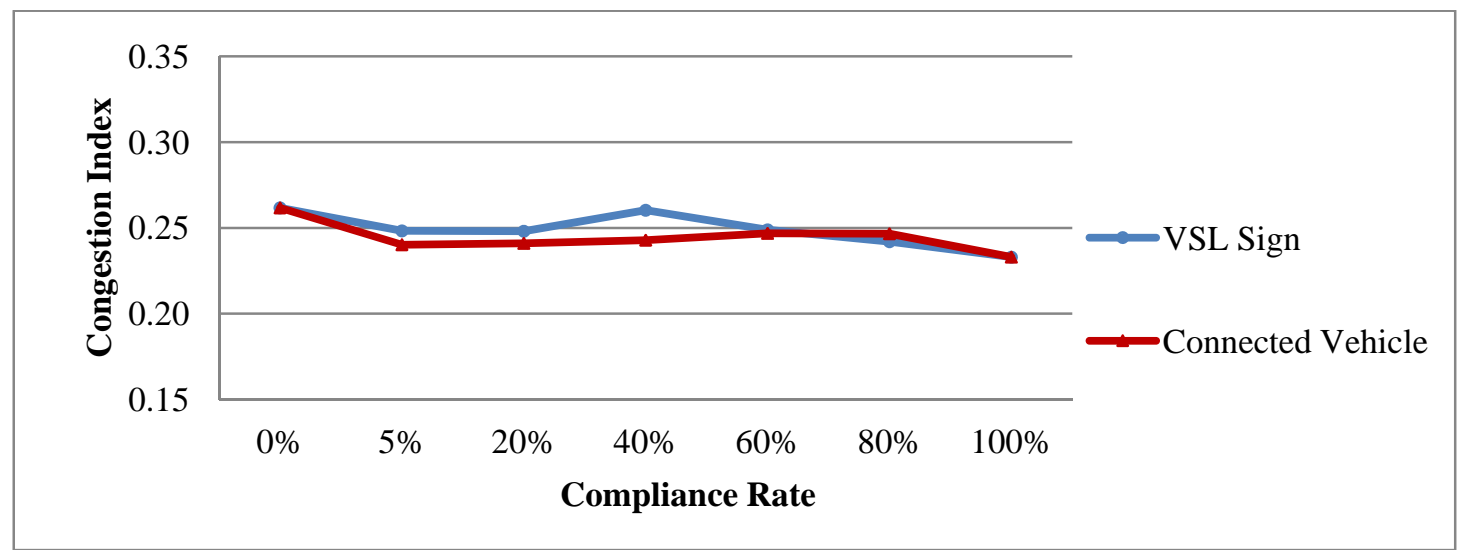

(a) Congestion index during entire study period

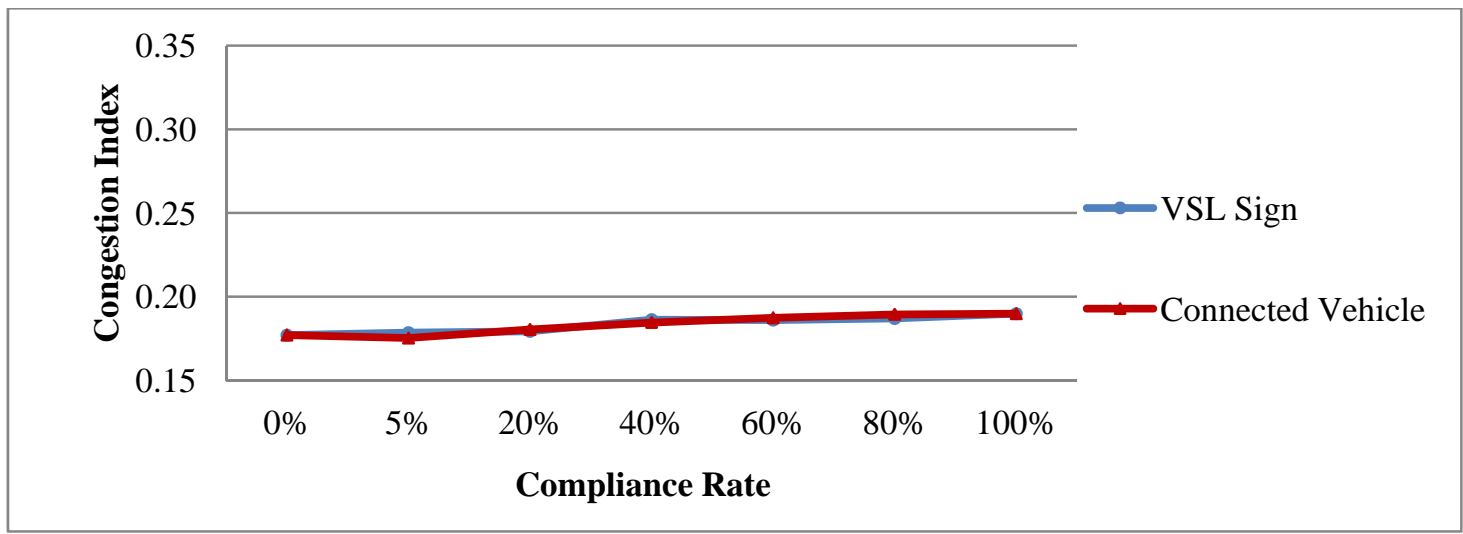

(b) Congestion index during uncongested conditions

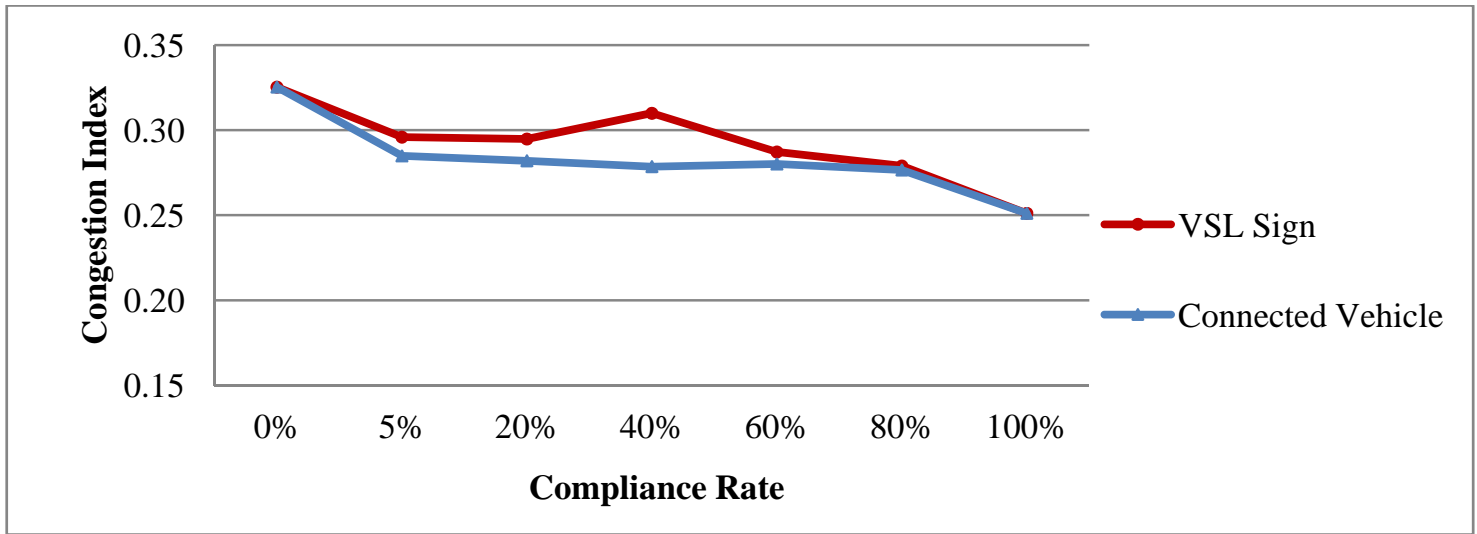

(c) Congestion index during congested conditions

Figure 5-10: Comparison of congestion index based on different compliance rate 
Table 5-13: Breakdown conditions at bottleneck, different market penetration using VSL sign

\begin{tabular}{lcccccc}
\hline Scenario & $\begin{array}{c}\text { Start } \\
\text { time } \\
\text { (hh:mm) }\end{array}$ & $\begin{array}{c}\text { Duration } \\
\text { (hh:mm) }\end{array}$ & $\begin{array}{c}\text { Speed Before } \\
\text { Breakdown } \\
\text { (mph) }\end{array}$ & $\begin{array}{c}\text { Maximum } \\
\text { Pre-Breakdown } \\
\text { Flow } \\
\text { (veh/hr) }\end{array}$ & $\begin{array}{c}\text { Breakdown } \\
\text { Speed } \\
\text { (mph) }\end{array}$ & $\begin{array}{c}\text { Queue } \\
\text { Discharge } \\
\text { (veh/hr) }\end{array}$ \\
\hline $0 \%$ & $16: 52$ & $1: 13$ & 50.91 & 8057 & 20.90 & 7728 \\
$5 \%$ & $16: 56$ & $1: 02$ & 48.07 & 8053 & 21.14 & 7752 \\
$20 \%$ & $17: 01$ & $0: 55$ & 46.23 & 8042 & 21.64 & 7712 \\
$40 \%$ & $17: 01$ & $0: 50$ & 43.41 & 8067 & 24.13 & 7733 \\
$60 \%$ & $17: 02$ & $0: 43$ & 40.10 & 8098 & 25.62 & 7773 \\
$80 \%$ & $17: 02$ & $0: 42$ & 40.63 & 8127 & 25.01 & 7765 \\
$100 \%$ & $17: 10$ & $0: 30$ & 38.98 & 8352 & 28.39 & 7935 \\
\hline
\end{tabular}

Table 5-14: Breakdown conditions at bottleneck, different market penetration using Connected Vehicle

\begin{tabular}{lcccccc}
\hline Scenario & $\begin{array}{c}\text { Start } \\
\text { time } \\
\text { (hh:mm) }\end{array}$ & $\begin{array}{c}\text { Duration } \\
\text { (hh:mm) }\end{array}$ & $\begin{array}{c}\text { Speed Before } \\
\text { Breakdown } \\
\text { (mph) }\end{array}$ & $\begin{array}{c}\text { Maximum } \\
\text { Pre-Breakdown } \\
\text { Flow } \\
\text { (veh/hr) }\end{array}$ & $\begin{array}{c}\text { Breakdown } \\
\text { Speed } \\
\text { (mph) }\end{array}$ & $\begin{array}{c}\text { Queue } \\
\text { Discharge } \\
\text { (veh/hr) }\end{array}$ \\
\hline $0 \%$ & $16: 52$ & $1: 13$ & 50.91 & 8057 & 20.90 & 7728 \\
$5 \%$ & $16: 56$ & $1: 02$ & 48.82 & 8048 & 21.02 & 7743 \\
$20 \%$ & $17: 01$ & $1: 01$ & 46.48 & 8042 & 20.94 & 7712 \\
$40 \%$ & $17: 01$ & $0: 55$ & 44.32 & 8091 & 22.80 & 7833 \\
$60 \%$ & $17: 02$ & $0: 42$ & 41.29 & 8062 & 24.12 & 7798 \\
$80 \%$ & $17: 03$ & $0: 36$ & 40.43 & 8093 & 26.11 & 7845 \\
$100 \%$ & $17: 08$ & $0: 33$ & 39.78 & 8272 & 28.09 & 7855 \\
\hline
\end{tabular}

Tables 5-13 and 5-14 show that using Connected Vehicle produces slightly better results at the bottleneck, compared to using the VSL signs. Both tables indicate that as the compliance rate decreases, the duration of breakdown increases. The breakdown starts sooner, compared to the $100 \%$ compliance rate and the speed during breakdown, which decreases. 


\subsection{Summary}

In this section, results of the simulation model's calibration, along with considering breakdown characteristics as part of the procedure, are presented and discussed. Later, performance of shockwave-based VSL is compared to VSL systems with a different fixed VSL message sign location-based congestion index, along with maximum back of queue and breakdown characteristics. The results show that shockwave-based systems outperform other VSL systems, and it can considerably decrease maximum back of queue and duration of breakdown while increasing average speed during breakdown. In addition, one of the important issues in implementing VSLs is whether drivers will obey the speed limit signs. Sensitivity analysis is conducted on VSL system performance based on different compliance rates that also consider informing drivers by VSL signs, or by using Connected Vehicle technology to disseminate information directly to each vehicle. Results indicate that as compliance rates increase, the VSL system is more successful. And, even for low compliance rates, the VSL system improves traffic mobility at bottlenecks. 


\section{CHAPTER 6}

\section{RESEARCH SUMMARY}

This study developed and assessed VSL strategies to reduce the adverse impacts of traffic flow breakdown at recurrent bottlenecks. The study developed VSL strategies to dynamically adjust the speed limits based on infrastructure detector measurements. These speed limits are sent to motorists on specific segments of the road via dynamic message signs.

Since the successful development and assessment of the VSL system directly depend on the quality of the utilized calibrated simulation model in these processes, this study developed a calibration methodology that not only considers field capacity, volume, and system performance values (as has been done traditionally), but also considers traffic flow breakdown parameters.

In addition to the infrastructure detector data-based VSL, VSL strategies were also developed to use Connected Vehicle technologies as a source of more detailed traffic data. Aside from using roadside equipment to inform drivers about posted speed limits, this study considers Connected Vehicle technologies to transfer VSL information to individual vehicles.

This chapter summarizes the contributions of the proposed methodology and conclusions of the study, and discusses the direction of future work.

\subsection{Study Contribution}

The main contributions of this study are as follows: 
- The study developed a new calibration approach that considers the breakdown characteristics calibration process. This process extends existing calibration procedures that are based on other measures and is critical in utilizing simulation for the reliable assessment of ADTM strategies.

- There are different definitions for breakdown occurrence. Estimating breakdown characteristics depends on these definitions, which are not systematic and tend to be subjective. In addition, traffic data has noise, which reduces the accuracy of estimated breakdown characteristics, such as the starting time of breakdown and its duration. This study used the wavelet transform methodology to identify the start and end time of breakdown occurrence. This approach allows for the estimation of other breakdown characteristics based on the estimated start and end time of breakdown occurrence.

- In the literature, researchers mainly focused on VSL applications during congestion. This dissertation developed and assessed strategies before and after traffic breakdown occurrence.

- The method was developed to determine the thresholds required to implement a VSL system that uses a heuristic switching logic-based controller to select the speed limit. To figure out the optimal thresholds, the first step involved finding the range of candidate thresholds using the probability of the breakdown graph and fundamental diagram. Then, by exhaustive enumeration on different sets of thresholds, the optimal thresholds were found.

- The shockwave-based VSL system is used in this research instead of using fixed location VSL strategies. In the shockwave-based approach, the location of the 
VSL influence area is pushed upstream as congestion propagates upstream so that the congestion will not propagate beyond the VSL influence area. This study compares the performance of shockwave-based VSL and the VSL systems with a fixed VSL influence area.

- In the developed strategy, during breakdown conditions, the VSL system is deactivated at the head of the congested area in order to increase the capacity of the freeway. The effects of VSL deactivation at head of the congested area are studied in this dissertation.

- This dissertation investigates the use of Connected Vehicle technology to support VSL. Using statistical methods, the minimum market penetration is found in order to obtain reliable traffic data. The VSL system was developed based on Connected Vehicle data by using the wavelet transform method to identify the location of congestion.

- In addition to VSL signs, this dissertation considers using Connected Vehicle technology to disseminate information directly to drivers. Under different compliance rates, the effects of using Connected Vehicle instead of VSL signs were studied.

\subsection{Conclusions}

Active Traffic and Demand Management (ATDM) strategies such as variable speed limit (VSL) strategies are state-of-the-art methods that are increasingly being considered to improve the efficiency of the existing freeway system. VSL strategies dynamically identify and disseminate the appropriate speed limits based on prevailing 
traffic conditions, road surface conditions, and weather conditions. Although the traffic safety benefits of implementing the VSL system are well-established, very few of the previously developed VSL strategies documented improvements on traffic mobility. This dissertation develops a shockwave-based VSL system with a time-variant VSL influence area that uses a heuristic switching logic-based controller with specified thresholds of prevailing traffic flow conditions. This VSL strategy aims to improve operations and mobility at critical bottlenecks. Before breakdown occurrence, the proposed VSL objective is to postpone or eliminate breakdown by decreasing the inflow at the bottleneck and achieving a uniform distribution in speed and flow. After breakdown, the VSL system aims to dampen the congestion by reducing the inflow traffic to the congested area and increasing the bottleneck capacity by deactivating the VSL at the head of the congested area. The shockwave-based VSL system pushes the VSL influence area location upstream as the congested area propagates upstream. In addition, Connected Vehicle technology provides a dynamic exchange of messages between the vehicles and infrastructure, which allows the VSL system to have access to much more detailed traffic data. This dissertation also investigates the use of Connected Vehicle data instead of detector data on VSL system performance. In this system, wavelet transform is used to analyze aggregated individual vehicle speed data to determine the location of congestion.

The developed VSL algorithms were assessed using a CORSIM microscopic simulation model. Without calibration of the simulation model, there is no assurance that the model's outputs are reliable or that the model will correctly predict traffic performance improvements. Traffic simulation models are widely and increasingly used in the transportation engineering field. The current methods of calibrating simulation 
models is generally based on capacity, volume, and system performance values, and do not take traffic breakdown characteristics into consideration. However, since the proposed VSL strategies are countermeasures to the impacts of breakdown conditions, considering breakdown characteristics in the calibration procedure is important in order to have a reliable assessment. Several enhancements were proposed in this study, including using the wavelet transform to determine the start and end time of breakdown occurrence and account for the breakdown characteristics at bottleneck locations in the calibration process.

In this dissertation, the performance of shockwave-based VSL with a time-variant influence area is compared to VSL systems with a fixed VSL influence area based on the congestion index, maximum back of queue, and breakdown characteristics. The results show that the shockwave-based VSL outperforms other VSL systems, and can considerably decrease the maximum back of queue and duration of breakdown while increasing the average speed during breakdown. In addition, one of the most important issues in implementing VSLs is whether or not drivers will obey the speed limit signs. Sensitivity analysis results indicate that as compliance rates increase, the VSL system is more successful. However, even for low compliance rates, the VSL system can improve traffic mobility at bottlenecks.

\subsection{Future Work}

This dissertation developed the VSL system, which was assessed by the microscopic simulation model. This section presents research opportunities for extending the scope of this dissertation: 
- This study considered 1-day field data and calibrated the simulation based on this data. As the statistical tests indicate, the breakdown characteristic values at different calibration runs are not significantly different from real-world data. To advance this procedure, calibration of the simulation based on different demands (days) with consideration of breakdown characteristics is required.

- This study showed that due to the stochastic nature of the simulation model, the stochasticity of breakdown characteristics can be modeled. The next step is to calibrate the simulation model based on the probability of breakdown in order to reflect real-world condition variations.

- The proposed VSL system is based on a reactive approach that uses current traffic conditions to identify traffic flow conditions, as well as on the selection of the speed limit. The next step is to develop a predictive VSL system, which uses current traffic conditions and a prediction module to identify traffic flow conditions, as well as selection of the speed limit.

- As part of this dissertation, Connected Vehicle technology is simulated using the CORSIM RTE interface. Connected Vehicle offers more detailed traffic information. This information can be used for a better understanding of traffic conditions, as well as to find new ADTM strategies. 


\section{REFERENCES}

Abdel-Aty, M., and Dhindsa, A. (2007). Coordinated Use of Variable Speed Limits and Ramp Metering for Improving Traffic Safety on Congested Freeways. In Proceedings of the $86^{\text {th }}$ Annual Meeting of Transportation Research Board, Washington, D.C.

Abdel-Aty, M., Dilmore, J., and Dhindsa, A. (2006). Evaluation of Variable Speed Limits for Real-Time Freeway Safety Improvement. Accident Analysis and Prevention, Vol. 38, pp. 335-345.

Adeli, H., and Jiang, X. (2008). Intelligent Infrastructure: Neural Networks, Wavelets, and Chaos Theory for Intelligent Transportation Systems and Smart Structures. CRC Press.

Allaby, P., Hellinga, B., and Bullock, M. (2007). Variable Speed Limits: Safety and Operational Impacts of a Candidate Control Strategy for Freeway Applications. IEEE Transactions on Intelligent Transportation System, Vol.8, pp. 671-680.

Ban, X., Chu, L., and Benouar, H. (2007). Bottleneck Identification and Calibration for Corridor Management Planning. Transportation Research Record: Journal of the Transportation Research Board, Vol. 1999, pp. 40-53.

Banks, J. (1991). The Two-Capacity Phenomenon: Some Theoretical Issues. Transportation Research Record: Journal of the Transportation Research Board, Vol. 1320, pp. 234-241.

Bloomberg, L., Swenson, M., and Haldors, B. (2003). Comparison of Simulation Models and the Highway Capacity Manual. In Proceedings of the $82^{\text {nd }}$ Annual Meeting of Transportation Research Board, Washington, D.C.

Brilon, W. (2005). Randomness and Reliability in Freeway Traffic Flow. Technical Report, TRAIL Research School, Delft, Netherlands.

Brewer, M., Pesti, G., and Schneider, W. (2005). Effectiveness of Selected Devices on Improving Work Zone Speed Limit Compliance. Technical Report, Texas Transportation Institute, Texas A\&M University, College Station, Texas.

Buckley, D., and Yagar, S. (1974). Capacity Funnels near on-Ramps. In Proceedings of the $6^{\text {th }}$ International Symposium on Transportation and Traffic Theory, American Elsevier Publishing Company, New York.

Carlson, R.C., Papamichail, I., Papageorgiou, M., and Messmer, A. (2010). Variable Speed Limits as a Mainline Metering Device for Freeways. In Proceedings of the $89^{\text {th }}$ Annual Meeting of Transportation Research Board, Washington, D.C. 
Cassidy, M., and Bertini, R. (1999). Some Traffic Features at Freeway Bottlenecks. Transportation Research Part B, Vol. 33, pp. 25-42.

Chen, C., Skabardonis, A., and Varaiya, P. (2004). Systematic Identification of Freeway Bottlenecks. Transportation Research Record: Journal of the Transportation Research Board, Vol. 1867, pp. 46-52.

Chu, L., and Liu, H. (2004). A Calibration Procedure for Microscopic Traffic Simulation. In Proceedings of the $83^{\text {rd }}$ Annual Meeting of Transportation Research Board, Washington, D.C.

Christofa, E., Argote, J., and Skabardonis, A. (2013). Arterial Queue Spillback Detection and Signal Control Based on Connected Vehicle Technology. In Proceedings of the $92^{\text {nd }}$ Annual Meeting of Transportation Research Board, Washington, D.C.

Comert, G., and Cetin, M. (2009). Queue Length Estimation from Probe Vehicle Location and the Impacts of Sample Size. European Journal of Operational Research, Vol. 197, pp. 196-202.

CTC and Associates LLC. (2003). Variable Speed Limit Signs for Winter Weather. Transportation Synthesis Report, Bureau of Highway Operations, Division of Transportation Infrastructure Development, Wisconsin Department of Transportation. (CTC and Associates LLC, http://www.ctcandassociates.com/index.html).

Daganzo, C. (2002). A Behavioral Theory of Multi-Lane Traffic Flow. Part II: Merges and the Onset of Congestion. Transportation Research Part B, Vol. 36, pp. 159-169.

Daganzo, C., Cassidy, M., and Bertini, R. (1999). Possible Explanations of Phase Transitions in Highway Traffic. Transportation Research Part A, Vol. 33, pp. 365-379.

Dion, F., Oh, J., and Robinson, R. (2011). Virtual Testbed for Assessing Probe Vehicle Data in IntelliDrive Systems. IEEE Transactions on Intelligent Transportation Systems, Vol. 12, pp. 635-644.

Dion, F., Robinson, R., and Oh, J. (2010). Evaluation of Usability of IntelliDrive Probe Vehicle Data for Transportation Systems Performance Analysis. Journal of Transportation Engineering, Vol. 137, pp. 174-183.

Donoho, D.L. (1993). Unconditional Bases are Optimal Bases for Data Compression and for Statistical Estimation. Applied and Computational Harmonic Analysis, Vol. 1, pp. 100-115.

Dowling, R., Skabardonis, A., and Alexiadis, V. (2004). Traffic Analysis Toolbox Volume III: Guidelines for Applying Traffic Micro-Simulation Modeling Software. Final Report, 
FHWA-HRT-04-040, U.S. Department of Transportation, Federal Highway Administration.

Dowling, R., Skabardonis, A., and Halkias, J. (2004). Guidelines for Calibration of Micro-Simulation Models Framework and Applications. Transportation Research Record: Journal of the Transportation Research Board, Vol. 1876, pp. 1-9.

Dowling, R., Holland, J., and Huang, A. (2002). Guidelines for Applying Traffic Micro-Simulation Modeling Software. California Department of Transportation, Oakland, California.

Elefteriadou, L., Letter, C., and Mintsis, E. (2012). Managed Lane Operations - Adjusted Time of Day Pricing vs. Near-Real Time Dynamic Pricing, Volume II: Ramp Signaling and Variable Speed Limits (VSL). Final Report, No. UF Project 00081551, Prepared for FDOT by the University of Florida Transportation Research Center, Gainesville, FL.

Elefteriadou, L., Martin, B., Simmerman, T., and Hale, D. (2011). Using Micro-Simulation to Evaluate the Effects of Advanced Vehicle Technologies on Congestion. Final Report, Prepared for Center for Multimodal Solutions for Congestion Mitigation by the University of Florida Transportation Research Center, Gainesville, FL.

Elefteriadou, L., Kondyli, A., Brilon, W., Jacobson, L., Hall, F., and Persaud, B. (2011). Proactive Ramp Management under the Threat of Freeway Flow Breakdown. Procedia-Social and Behavioral Sciences, Vol. 16, pp. 4-14.

Elefteriadou, L., and Lertworawanich, P. (2003). Defining, Measuring and Estimating Freeway Capacity. In Proceedings of the $82^{\text {nd }}$ Annual Meeting of Transportation Research Board, Washington, D.C.

Elefteriadou, L., Roess, R., and McShane, W. (1995). The Probabilistic Nature of Breakdown at Freeway-Merge Junctions. Transportation Research Record: Journal of the Transportation Research Board, Vol. 1484, pp. 80-89.

Federal Highway Administration. (2000). Manual on Uniform Traffic Control Devices. U.S. Department of Transportation, Washington, D.C.

Fellendorf, M., and Vortisch, P. (2001). Validation of the Microscopic Traffic Flow Model VISSIM in Different Real-World Situations. In Proceedings of the $80^{\text {th }}$ Annual Meeting of Transportation Research Board, Washington, D.C.

Gazis, D., and Herman, R. (1992). The Moving and 'Phantom' Bottlenecks. Transportation Science, Vol. 26, pp. 223-229. 
Geistefeldt, J. (2008). Empirical Relation between Stochastic Capacities and Capacities Obtained from the Speed-Flow Diagram. In Proceedings of Symposium on the Fundamental Diagram: 75 Years, Woods Hole, MA.

Ghods, A., Kian, A., and Tabibi, M. (2009). Adaptive Freeway Ramp Metering and Variable Speed Limit Control: A Genetic-Fuzzy Approach. Intelligent Transportation Systems Magazine, IEEE, vol. 1, pp. 27-36.

Gomes, G., May, A., and R. Horowitz, (2004). A Micro-Simulation Model of a Congested Freeway Using VISSIM. In Proceedings of the $83^{\text {rd }}$ Annual Meeting of Transportation Research Board, Washington, D.C.

Graves, T., Karr, A., Rouphail, N., and Takuriah, P. (1998). Real-Time Prediction of Incipient Congestion on Freeways from Detector Data. NISS Technical Report. http://www.niss.org/sites/default/files/pdfs/technicalreports/tr79.pdf, Accessed November 25, 2012.

Haas, R., Carter, M., Perry, E., Trombly, J., Bedsole, E., and Margiotta, R. (2009). iFlorida Model Deployment. Final Evaluation Report. Final Report, FHWA-HOP-08-050, U.S. Department of Transportation, Federal Highway Administration.

Halkias, B., Kopelias, P., Papandreou, K., Politou, A., Prevedouros, P., and Skabardonis, A. (2007). Freeway Bottleneck Simulation, Implementation and Evaluation. Transportation Research Record: Journal of the Transportation Research Board, Vol. 2012, pp. 84-93.

Harbord, B. (1995). Application of SISTM to Dynamic Control on the M25. IEE Colloquium on Dynamic Control of Strategic Inter-Urban Road Networks. http://digital-library.theiet.org/content/conferences/10.1049/ic_19950246, Accessed November 25, 2012.

Henclewood, D., Suh, W., Rodgers, M., Hunter, M., and Fujimoto, R. (2012). A Case for Real-time Calibration of Data-driven Microscopic Traffic Simulation Tools. In Proceedings of the Winter Simulation Conference, IEEE.

Hegyi, A., and Hoogendoorn, S.P. (2010). Dynamic Speed Limit Control to Resolve Shockwaves on Freeways - Field Test Results of the SPECIALIST Algorithm. In Proceedings of the $13^{\text {th }}$ International IEEE ITS, Madeira Island, Portugal.

Hegyi, A., De-Schutter, B., and Hellendoorn, J. (2005). Model Predictive Control for Optimal Coordination of Ramp Metering and Variable Speed Limits. Transportation Research Part C, Vol. 13, pp. 185-209. 
Hegyi, A., De-Schutter, B., and Hellendoorn, J. (2003). MPC-based Optimal Coordination of Variable Speed Limits to Suppress Shock Waves in Freeway. In Proceedings of the American Control Conference, Denver, Colorado, pp. 4083-4088.

Holm, P., Tomich, D., Sloboden, J., and Lowrance, C. (2007). Traffic Analysis Toolbox Volume IV: Guidelines for Applying CORSIM Micro-Simulation Modeling Software. Final Report, FHWA-HOP-07-079, U.S. Department of Transportation, Federal Highway Administration.

Hourdakis, J., Michalopoulos, P., and Kottommannil, J. (2003). Practical Procedure for Calibrating Microscopic Traffic Simulation Models. Transportation Research Record: Journal of the Transportation Research Board, Vol. 1852, pp. 130-139.

Hollander, Y., and Liu, R. (2008). The Principles of Calibrating Traffic Micro-Simulation Models. Transportation, Vol. 35, pp. 347-362.

Iwasaki, M. (1991). Empirical Analysis of Congested Traffic Flow Characteristics and Free Speed Affected by Geometric Factors on an Intercity Expressway. Transportation Research Record: Journal of the Transportation Research Board, Vol. 1320, pp. 242-250.

Kattan, L., and Saidi, S. (2013). Comparative Analysis of Probe-based ramp metering with Detector-based and Pre-timed Ramp Metering. Journal of Advanced Transportation, Vol. 47, pp: 61-78.

Kerner, B. (1998). Experimental Features of Self-Organization in Traffic Flow. Physical Review Letters, Vol. 81, pp. 3797-3800.

Kianfar, J., and Praveen, E. (2013). Placement of Roadside Equipment (RSE) in Connected Vehicle Environment for Travel Time Estimation. In Proceedings of the $92^{\text {nd }}$ Annual Meeting of Transportation Research Board, Washington, D.C.

Kim, K., and Rilett, L. (2004). A Genetic Algorithm Based Approach to Traffic Micro-Simulation Calibration Using ITS Data. In Proceedings of the $83^{\text {rd }}$ Annual Meeting of Transportation Research Board, Washington, D.C.

Kohler, U. (1974). Stability of Vehicle Platoons. Transportation and traffic theory, Vol. 6, pp. 39-55.

Kondyli, A., Soria, I., Duret, A., and Elefteriadou, L., (2012). Sensitivity Analysis of CORSIM with Respect to the Process of Freeway Flow Breakdown at Bottleneck Locations. Simulation Modelling Practice and Theory, Vol. 22, pp. 197-206.

Kondyli, A., Elefteriadou, L., Brilon, W., Hall, F., Persaud, B., and Washburn, S. (2013). Development and Evaluation of Methods for Constructing Breakdown Probability Models. Journal of Transportation Engineering, Vol. 139, pp. 931-940. 
Kondyli, A. (2009). Breakdown Probability Model at Freeway-Ramp Merges Based on Driver Behavior. Ph.D. Dissertation, University of Florida, Gainesville, Fla.

Koshi, M., Iwasaki, M., and Ohkura, I. (1983). Some Findings and an Overview on Vehicular Flow Characteristics. In Proceedings of the $8^{\text {th }}$ International Symposium on Transportation and Traffic Theory, Ontario, Canada.

Kuhne, R., Mahnke, R., and Hinkel, J. (2006). Modeling the Effects of Corridor Control Systems on Road Capacity. In Proceedings of the $5^{\text {th }}$ International Symposium on Highway Capacity and Quality of Service, Japan Society of Traffic Engineers, Yokohama, Japan, pp. 289-298.

Lee, J., Park, B., Won, J., and Yun, I. (2013). A Simplified Procedure for Calibrating Microscopic Traffic Simulation Models. In Proceedings of the $92^{\text {nd }}$ Annual Meeting of Transportation Research Board, Washington, D.C.

Lee, J., and Ozbay, K. (2009). New Calibration Methodology for Microscopic Traffic Simulation Using Enhanced simultaneous perturbation stochastic approximation approach. Transportation Research Record: Journal of the Transportation Research Board, Vol. 2124, pp.233 -240.

Lee, C., Hellinga, B., and Saccomanno, F. (2004). Assessing safety benefits of variable speed limits. Transportation Research Record: Journal of the Transportation Research Board, Vol.1897, pp. 183-190.

Lenz, H., Sollacher, R., and Lang, M. (1999). Nonlinear Speed-Control for a Continuum Theory of Traffic Flow. In Proceedings of the $14^{\text {th }}$ World Congress of IFAC, Beijing, China.

Li, j., Zhou, K., Shladover, S., and Skabardonis, A. (2013). Estimating Queue Length under the Connected Vehicle Technology: Using Probe Vehicle, Loop Detector, and Fused Data. In Proceedings of the $92^{\text {nd }}$ Annual Meeting of Transportation Research Board, Washington, D.C.

Liao, C., and Davis, G. (2007). Simulation Study of a Bus Signal Priority Strategy Based on GPS/AVL and Wireless Communications. Transportation Research Record: Journal of the Transportation Research Board, Vol. 2034, pp. 82-91.

Lin, P., Kang, K., and Chang, G. (2004). Exploring the Effectiveness of Variable Speed Limit Controls on Highway Work-Zone Operations. Intelligent Transportation Systems, Vol. 8, pp.1-14.

Lind, G. (2006). Weather and Traffic Controlled Variable Speed Limits in Sweden. Final Report Movea trafikkonsult AB, Stockholm, Sweden. 
Lu, X., and Skabardonis, A. (2007). Freeway Traffic Shockwave Analysis: Exploring the NGSIM Trajectory Data. In Proceedings of the $86^{\text {th }}$ Annual Meeting of Transportation Research Board, Washington, D.C.

Ma, J., Dong, H., and Zhang, M. (2007). Calibration of Micro-Simulation with Heuristic Optimization Methods. Transportation Research Record: Journal of the Transportation Research Board, Vol. 1999, pp. 208-217.

Menneni, S., Sun, C., and Vortisch, P. (2008). Micro-Simulation Calibration Using Speed-Flow Relationships. In Proceedings of the $87^{\text {th }}$ Annual Meeting of Transportation Research Board, Washington, D.C.

Meyer, Y., and Salinger, D. (1995). Wavelets and Operators. Cambridge University Press.

Muñoz, J.C., and Daganzo, C.F. (2003). Structure of the Transition Zone behind Freeway Queues. Transportation Science, Vol. 37, pp. 312-329.

Najm, W., Koopmann, J., Smith, J., and Brewer, J. (2010). Frequency of Target Crashes for IntelliDrive Safety Systems. No. HS-811 381, USDOT National Highway Traffic Safety Administration.

Ni, D., Li, J., Andrews, S., and Wang, H. (2011). A Methodology to Estimate Capacity Impact due to Connected Vehicle Technology. International Journal of Vehicular Technology, Vol. 2012, pp. 1-10.

Okamura, H., Watanabe, S., and Watanabe, T. (2000). An Empirical Study on the Capacity of Bottlenecks on the Basic Suburban Expressway Sections in Japan. In Proceedings of the $4^{\text {th }}$ International Symposium on Highway Capacity, TRB Circular E-C018, Transportation Research Board, Washington D.C., pp. 120-129.

Papageorgiou, M., Blosseville, J.M., and Hadj-Salem, H. (1998). La Fluidification Des Rocades De l'Ile De France: Un Projet D’importance. Technical report, No. 1998-17, Dynamic Systems and Simulation Lab., Technology University of Crete, Chania, Greece.

Papageorgiou, M., Kosmatopoulos, E., and Papamichail, I. (2008). Effects of Variable Speed Limits on Motorway Traffic Flow. Transportation Research Record: Journal of the Transportation Research Board, Vol. 2047, pp. 37-48.

Park, H., Bhamidipati, C., and Smith, B. (2011). Development and Evaluation of an Enhanced IntelliDriveSM Enabled Lane Changing Advisory Algorithm to Address Freeway Merge Conflict. In Proceedings of the $90^{\text {th }}$ Annual Meeting of Transportation Research Board, Washington, D.C.

Park, B., and Qi, H. (2005). Development and Evaluation of a Procedure for the 
Calibration of Simulation Models. Transportation Research Record: Journal of the Transportation Research Board, Vol. 1934, pp. 208-217.

Park, B., and Schneeberger, J. (2003). Microscopic Simulation Model Calibration and Validation: A Case Study of VISSIM for a Coordinated Actuated Signal System. In Proceedings of the $82^{\text {nd }}$ Annual Meeting of Transportation Research Board, Washington, D.C.

Paz, A., Molano, V., and Gaviria, C. (2012). Calibration of CORSIM Models Considering all Model Parameters Simultaneously. In Proceedings of the $15^{\text {th }}$ International IEEE Conference on Intelligent Transportation Systems Anchorage, Alaska.

PBS\&J. (2009). I-4 Variable Speed Limit Effectiveness Study. Prepared for the Florida Department of Transportation, District 5.

Persaud, B., Yagar, S., Tsui, D., and Look, H. (2001). Study of Breakdown-Related Capacity for a Freeway with Ramp Metering. Transportation Research Record: Journal of the Transportation Research Board, Vol. 1748, pp. 110-115.

Persaud, B., Yagar, S., and Brownlee, R. (1998). Exploration of the Breakdown Phenomenon in Freeway Traffic. Transportation Research Record: Journal of the Transportation Research Board, Vol. 1634, pp. 64-69.

Piao, J., and McDonald, M. (2008). Safety Impacts of Variable Speed Limits - A Simulation Study. In Proceedings of the $11^{\text {th }}$ International IEEE, Conference on Intelligent Transportation Systems, Beijing, China.

Rakha, H., Van-Aerde, M., Bloomberg, L., and Huang, X. (1998). Construction and Calibration of a Large-Scale Micro-Simulation Model of the Salt Lake Area. Transportation Research Record: Journal of the Transportation Research Board, Vol. 1644, pp. 93-102.

Rämä, P., Raitio, J., Anttila, V., and Schirokoff, A. (2001). Effects of Weather Controlled Speed Limits on Driver Behavior on a Two-Lane Road. International Conference: Traffic Safety on Three Continents, No. VTI Konferens 18A.

Rämä, P. (1999). Effects of Weather-Controlled Variable Speed Limits and Warning Signs on Driver Behavior. Transportation Research Record: Journal of the Transportation Research Board, Vol. 1689, pp. 53-59.

Rim, H., Oh, C., Kang, K., and Kim, S. (2011). Estimation of Lane-Level Travel Times in Vehicle-to-Vehicle and Vehicle-to-Infrastructure Based Traffic Information System. Transportation Research Record: Journal of the Transportation Research Board, Vol. 2243, pp. 9-16. 
Robinson, M. (2000). Examples of Variable Speed Application. Speed Management Workshop, Transportation Research Board 79th Annual Meeting, http://safety.fhwa.dot.gov/speedmgt/vslimits/docs/vslexamples.ppt, Accessed February 23, 2013.

Sarvi, M., Kuwahara, M., and Ceder, A. (2007). Observing Freeway Ramp Merging Phenomena in Congested Traffic. Journal of Advanced Transportation, Vol. 41, pp. 145-170.

Schultz, G., and Rilett, L. (2004). Analysis of Distribution and Calibration of Car-Following Sensitivity Parameters in Microscopic Traffic Simulation Models. Transportation Research Record: Journal of the Transportation Research Board, 1876, pp. 41-51.

Schrank, D., Lomax, T., and Eisele, B. (2011). Urban Mobility Report. Technical Report, Texas Transportation Institute, Texas A\&M University, College Station, Texas.

Shen, L. (2008). Freeway Travel Time Prediction System Using Dynamic Neural Networks. Ph.D. Dissertation, Florida International University, Miami, FL.

Shladover, S., and Kuhn, T. (2008). Traffic Probe Data Processing for Full-Scale Deployment of Vehicle-Infrastructure Integration. Transportation Research Record: Journal of the Transportation Research Board, Vol. 2086, pp. 115-123.

Sisiopiku, V. (2001). Variable Speed Control: Technologies and Practices. In Proceedings of the $11^{\text {th }}$ Annual Meeting of ITS America, Miami, FL.

Steel, P., McGregor, R., Guebert, A., and McGuire, T. (2005). Application of Variable Speed Limits along the Trans Canada Highway in Banff National Park. In Proceedings to the Annual Conference of the Transportation Associate of Canada, Canada.

Talebpour, A., Mahmassani, H., and Hamdar, S. (2013). Speed Harmonization: Effectiveness Evaluation under Congested Conditions. In Proceedings of the $82^{\text {nd }}$ Annual Meeting of Transportation Research Board, Washington, D.C.

Tignor, S., Brown, L., Butner, J., Cunard, R., Davis, S., Hawkins, H., Fischer, E., Kehrli, M., Rusch, P., and Wainwright, W. (1999). Innovative Traffic Control-Technology and Practice in Europe. International Technology Exchange Program Report, U.S. Department of Transportation.

Transportation Research Board. (2010). Highway Capacity Manual. Washington, DC.

Venkatanarayana, R., Park, H., Smith, B., Skerrit, C., and Ruhter, N. (2011). Application 
of IntelliDriveSM to Address Oversaturated Conditions on Arterials. In Proceedings of the $90^{\text {th }}$ Annual Meeting of Transportation Research Board ,Washington, D.C.

Wang, Y., and Ioannou, P. A. (2011). New Model for Variable Speed Limits. Transportation Research Record: Journal of the Transportation Research Board, Vol. 2249, pp. 38-43.

Willke, T., Tientrakool, P., and Maxemchuk, N. (2009). A Survey of Inter-Vehicle Communication Protocols and Their Applications. IEEE Communications Surveys Tutorials, Vol. 11, pp. 3-20.

Wisconsin Department of Transportation. (2002). Freeway System Operational Assessment-PARAMICS Calibration and Validation Guidelines. Technical Report I-33. Wisconsin DOT, District 2.

Young, R. (2010). Rural Variable Speed Limit for Southeast Wyoming. In Proceedings of the $89^{\text {th }}$ Annual Meeting of Transportation Research Board, Washington, D.C.

Yunlong, Z., and Owen, E. (2004). Systematic Validation of a Microscopic Traffic Simulation Program. Transportation Research Record: Journal of the Transportation Research Board, Vol. 1876, pp. 112-120.

Zarean, M., Pisano, P., Dirnberger, K., and Robinson, M., (1999). Variable Speed Limit Systems: The-State-Of-The-Practice. In Proceedings of the Rural Advanced Technology \& Transportation Systems Conference, Flagstaff, Arizona.

Zeng, X., Balke, K., and Songchitruksa, P. (2012). Potential Connected Vehicle Applications to Enhance Mobility, Safety, and Environmental Security. Technical report, Texas A\&M University System.

Zhang, L., Holm, P., and Colyar, J. (2004). Identifying and Assessing Key Weather-related Parameters and Their Impacts on Traffic Operations Using Simulation. FHWA-HRT-04-131, U.S. Department of Transportation, Federal Highway Administration.

Zhang, M., Ma, J., and Dong, H. (2008). Developing Calibration Tools for Microscopic Traffic Simulation Final Report Part II: Calibration Framework and Calibration of Local/Global Driving Behavior and Departure/Route Choice Model Parameters. Technical report, California Path Program, Institute of Transportation Studies, University of California, Berkeley.

Zhang, Y., and Owen, L.E. (2004). Systematic Validation of a Microscopic Traffic Simulation Program. In Proceedings of the $83^{\text {rd }}$ Annual Meeting of Transportation Research Board, Washington, D.C. 
Zheng, Z., Ahn, S., Chen, D., and Laval, J. (2011). Applications of Wavelet Transform for Analysis of Freeway Traffic: Bottlenecks, Transient Traffic, and Traffic Oscillations. Transportation Research Part B, Vol. 45, pp. 372-384. 


\section{APPENDIX A}

\section{DEVELOPED CODE OF VSL STRATEGY}

This appendix presents developed VSL strategy code in C++.

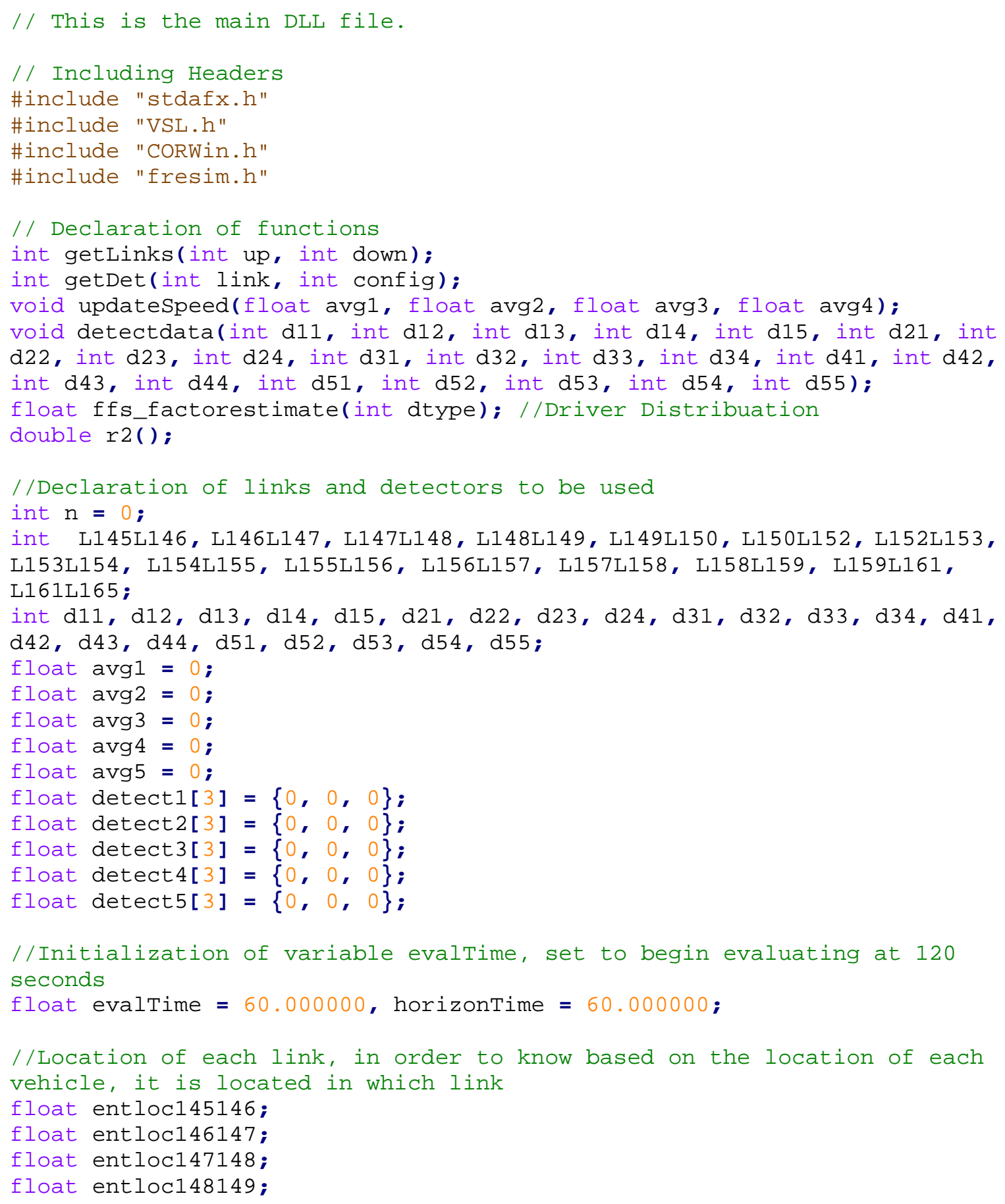


float entloc149150;

float entloc150152;

float entloc152153;

float entloc153154;

float entloc154155;

float entloc155156;

float entloc156157;

float entloc157158;

float entloc158159;

float entloc159161;

float entloc161165;

float enthresh;

//Compliance rate

double cp_threshold;

//Free-flow speed at each link prior to VSL strategy adjust it.

float s145146;

float s146147;

float s147148;

float s148149;

float s149150;

float s150152;

float s152153;

float s153154;

float s154155;

float s155156;

float s156157;

float s157158;

float s158159;

float s159161;

float s161165;

int flag_vehicle[100000];

int flag_vehicleid[100000];

int flag_count $=0$;

float ffs_factor $=1.0$;

FILE *VSL_results = NULL;

char text[Output];

DLL_EXPORT void_stdcall vsl_Initialize()\{

// Reassigning link numbers to match CORSIM Internal Link Numbering to node )

// User Defined Link Numbering getLinks(upstream node, downstream

$$
\begin{aligned}
& \text { L145L146 = getLinks }(145,146) ; \\
& \text { L146L147 = getLinks }(146,147) ; \\
& \text { L147L148 = getLinks }(147,148) ; \\
& \text { L148L149 = getLinks }(148,149) ; \\
& \text { L149L150 }=\operatorname{getLinks}(149,150) ; \\
& \text { L150L152 }=\operatorname{getLinks}(150,152) ; \\
& \text { L152L153 = getLinks }(152,153) ; \\
& \text { L153L154 = getLinks }(153,154) ; \\
& \text { L154L155 = getLinks }(154,155) ;
\end{aligned}
$$




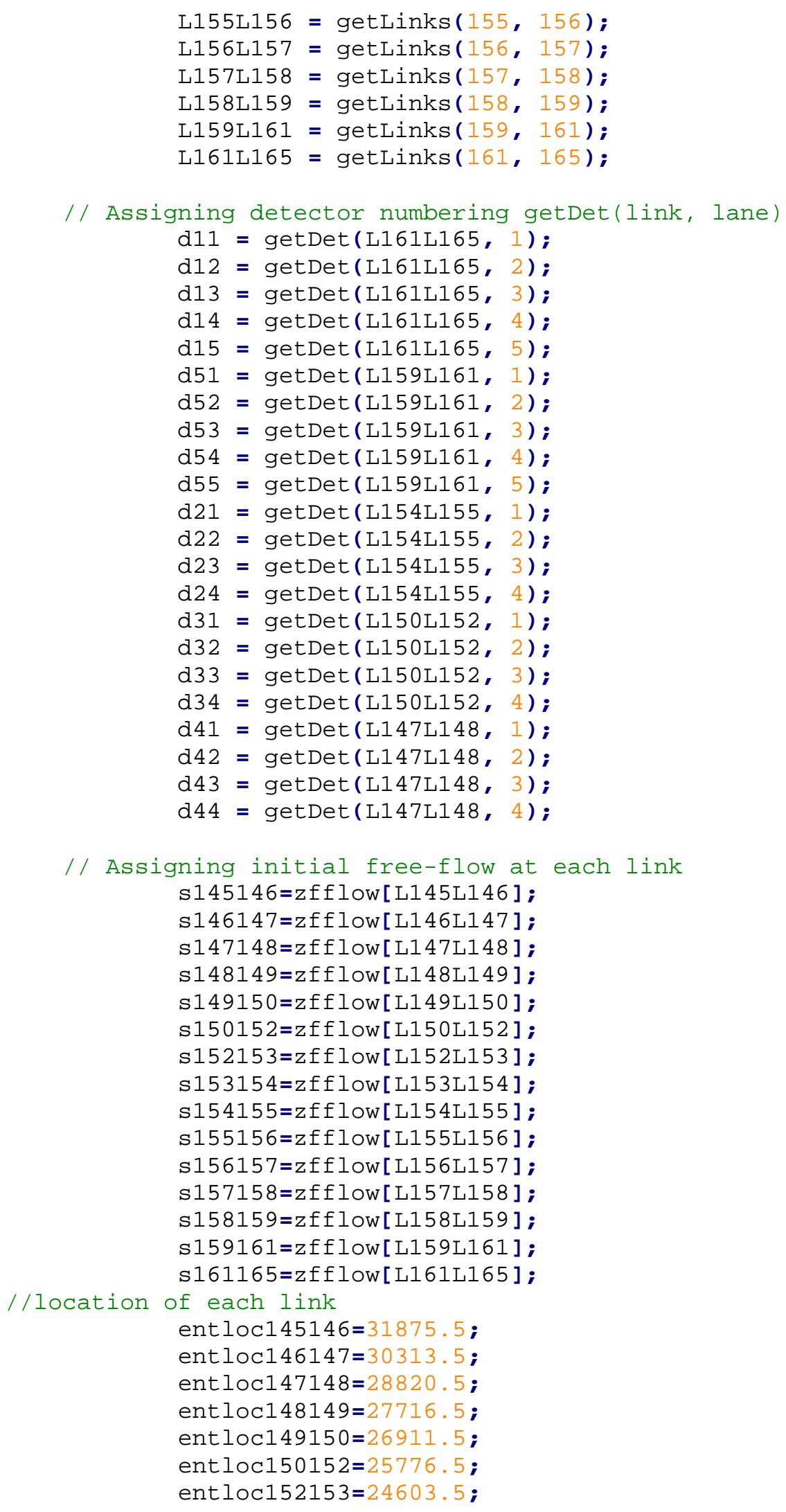

// Assigning initial free-flow at each link s145146=zfflow [L145L146] ; s146147=zfflow[L146L147]; s147148=zfflow [L147L148]; s148149=zfflow [L148L149]; s149150=zfflow [L149L150]; s150152=zfflow [L150L152]; s152153=zfflow [L152L153]; s153154=zfflow [L153L154]; s154155=zfflow [L154L155]; s155156=zfflow [L155L156]; s156157=zfflow [L156L157]; s157158=zfflow [L157L158]; s158159=zfflow [L158L159]; s159161=zfflow [L159L161];

//location of each link s161165=zfflow [L161L165];

entloc145146=31875.5; entloc146147=30313.5; entloc $147148=28820.5$; entloc148149=27716.5; entloc149150 $=26911.5$; entloc150152 $=25776.5$; entloc152153=24603. 5 ; 


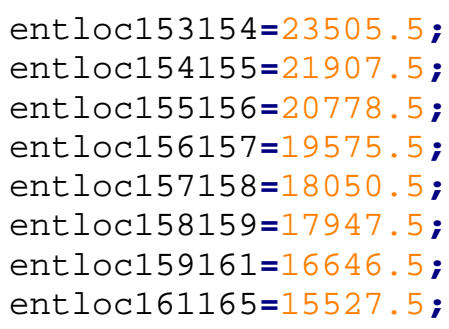

//Distance prior entering a link that VSL sign is not visible for drivers any more

enthresh=110.0;

// Setting the Point processing Interval

dppint $=60$;

cp_threshold=1.0;

if( fopen_s( \&VSL_results, "VSL_results.txt", "wt" ) !=○ )

\{

sprintf_s( text, "File not created for VSL. \n");

\}

Outputstring( text, Output, 2, 0 );

else

\{

sprintf_s( text, "New file was created for VSL. \n");

\}

OutputString( text, Output, 2, $\odot)$ );

\}

DLL_EXPORT void_stdcall vsl_PreFreesimVehicle()

\{

if (yinit $!=1)\{$

simulation is still in initialization period

//make sure

if (zclock == horizonTime) \{

// detector data

getteing updated each 5 minutes

horizonTime $=$ horizonTime +60 ;

detectdata(d11, d12, d13, d14, d15, d21, d22, d23, d24, d31, d32,

d33, d34, d41, d42, d43, d44, d51, d52, d53, d54, d55); \}

if (zclock == evalTime) \{

limit is evaluated every 5 minutes starting at time evalTime

// speed evalTime = evalTime + 180;

updatespeed(avg1, avg2, avg3, avg4);

if(VSL_results != NULL)

fprintf_s(VSL_results,"\%.2f \t \%.2f \t \%.2f \n\r", zclock, zfflow[L161L165]);

\}

//Finding the location of each vehicle, Based on Compliance rate it decides

whether to send new information to them or not

for (int index $=\odot$; index<ttlgvh; index++)

\{

//for each segment, the code checks to see if the vehicle is located there or not. 
if (vehlnk[index]-1==L145L146 \&\& fregvh[index]>0 \&\& drvtyp[index]>0 \&\& entloc145146-zvhdst [index]<=enthresh)

\{

int iveh_count $=\odot$;

bool flag_find=false;

while (flag_find==false \&\& iveh_count $<=f l a g \_c o u n t-1$ )

\{

if (flag_vehicleid[iveh_count]==fregvh[index])

\{

flag_find=true;

else

\{

iveh_count++;

\}

\}

if (flag_find==true) // if it is already selected and found in another segment, there is no need to change any thing.

\{

if (flag_vehicle[iveh_count]==1)

\{

zfspd[index]=s145146*ffs_factorestimate(drvtyp[index]);

\}

else

\{

zfspd[index]=zfflow[L145L146] *ffs_factorestimate(drvtyp[index] );

\}

\}

else

\{

if $\left(r 2()>c p \_t h r e s h o l d\right) / /$ based on compliance rate, assigns new speed limit or keep the initial one.

\{

zfspd[index]=s145146*ffs_factorestimate(drvtyp[index]); //Driver will

drive based on its characteristic and speed limit

flag_count=flag_count +1 ;

flag_vehicleid[flag_count -1$]=f r e g v h[$ index] ;

\}

flag_vehicle[flag_count-1]=1;

else

\{

zfspd[index]=zfflow[L145L146]*ffs_factorestimate(drvtyp[index]);

flag_count $=f l a g \_c o u n t+1$;

flag_vehicleid[flag_count -1$]=f r e g v h[$ index] ;

\}

flag_vehicle[flag_count -1$]=\odot$;

\}

\}

//the same calculation for the next segment.

if (vehlnk[index]-1==L146L147 \&\& fregvh[index]>0 \&\& drvtyp[index]>0 \&\& entloc146147-zvhdst [index]<=enthresh) 


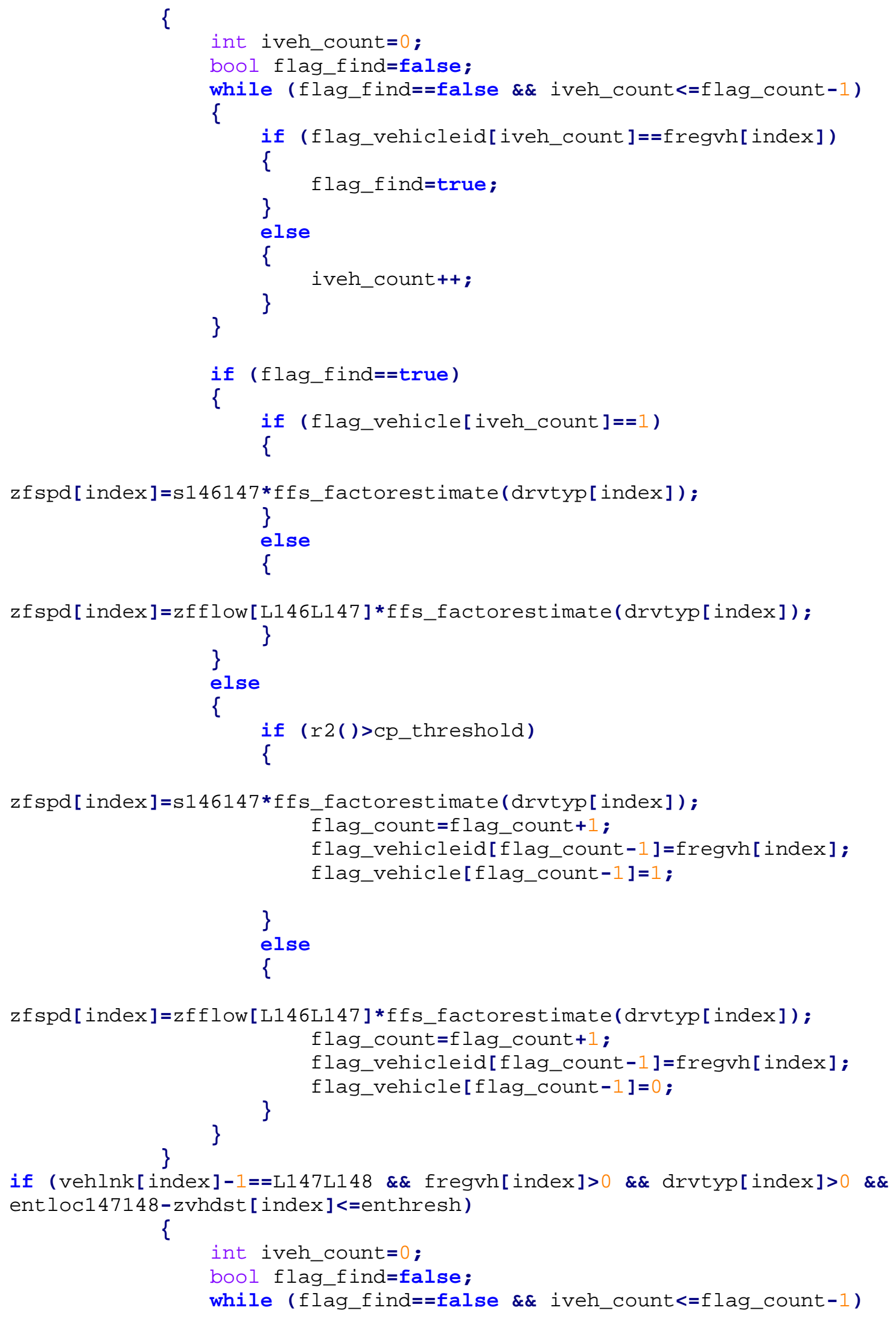




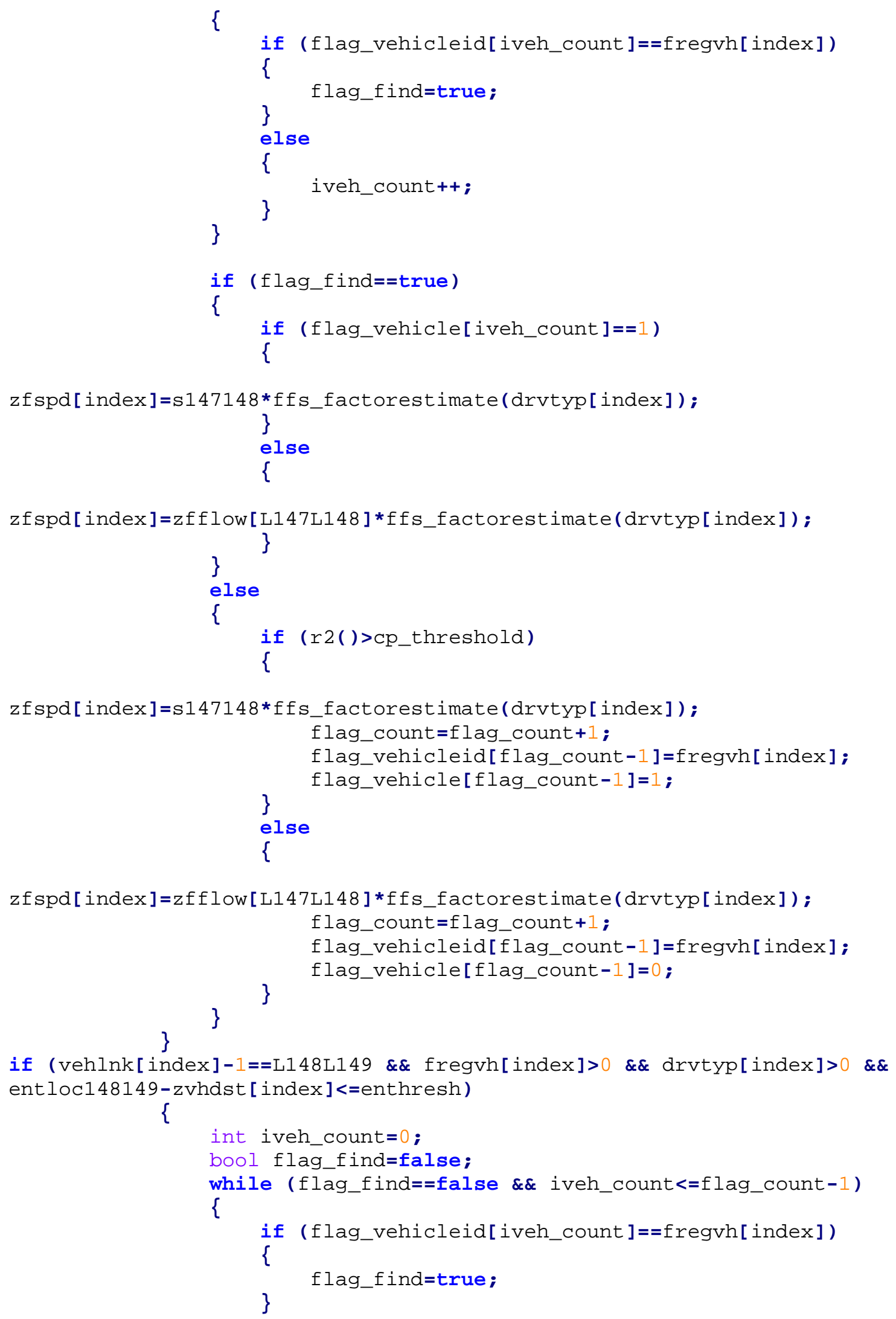




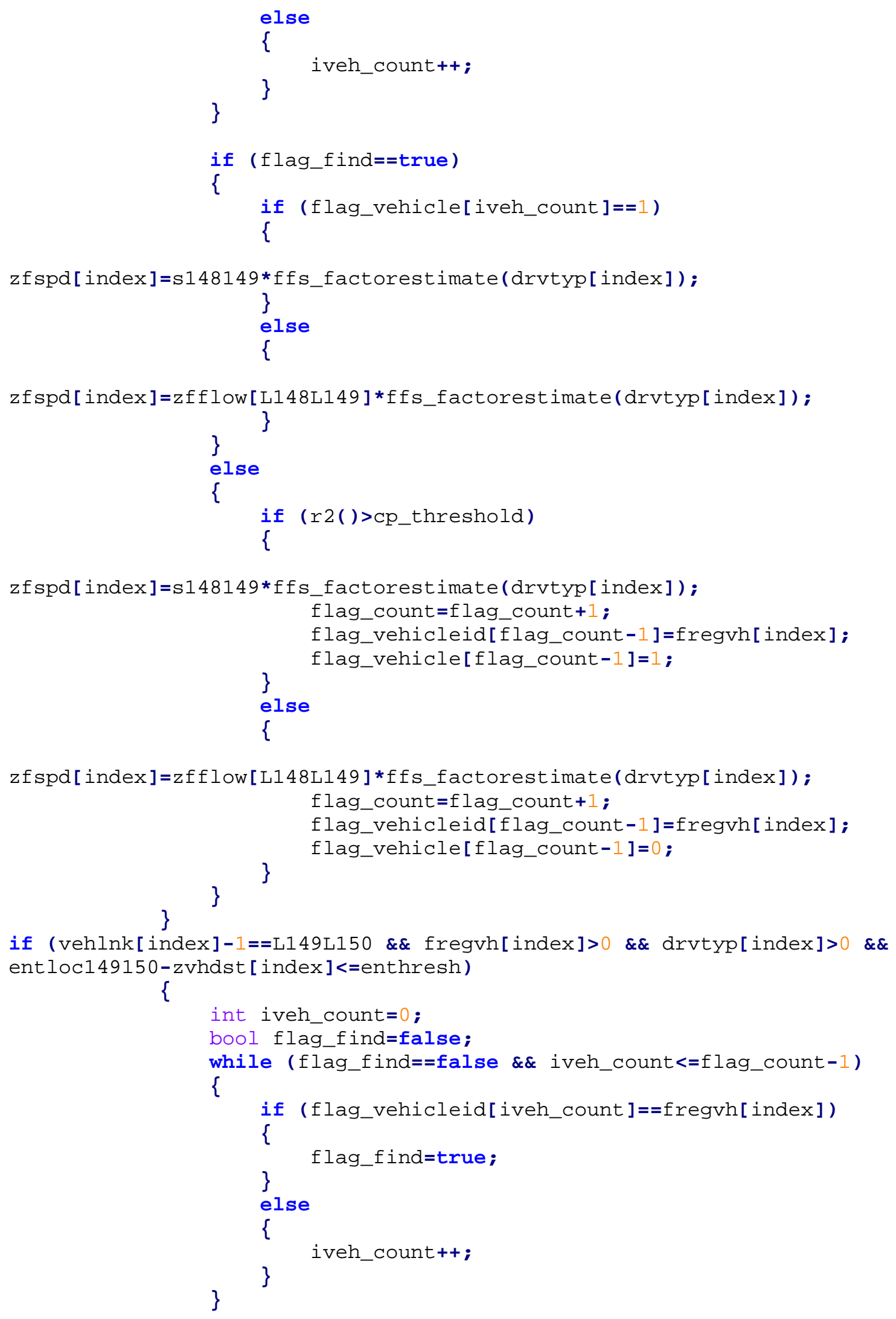




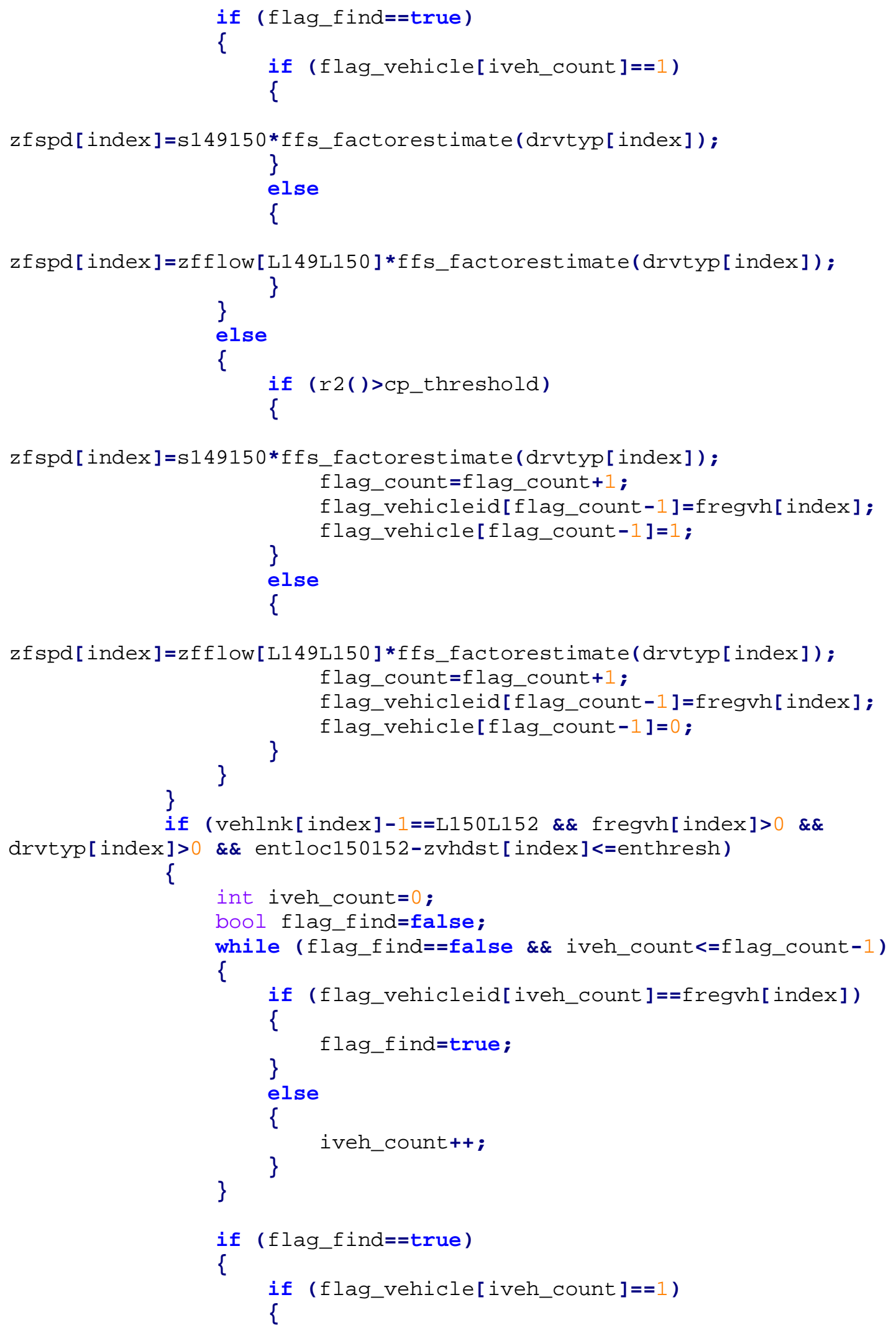




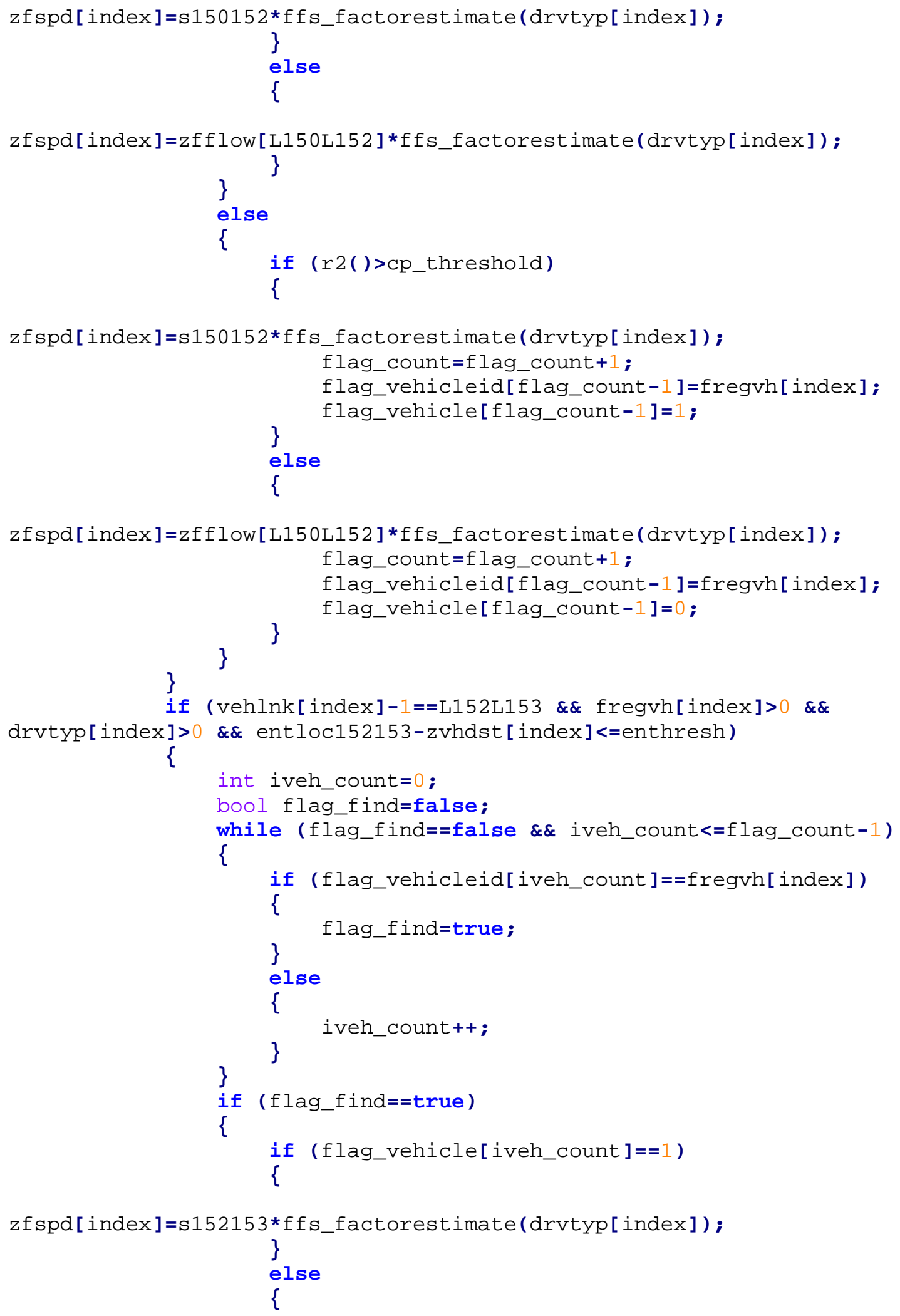









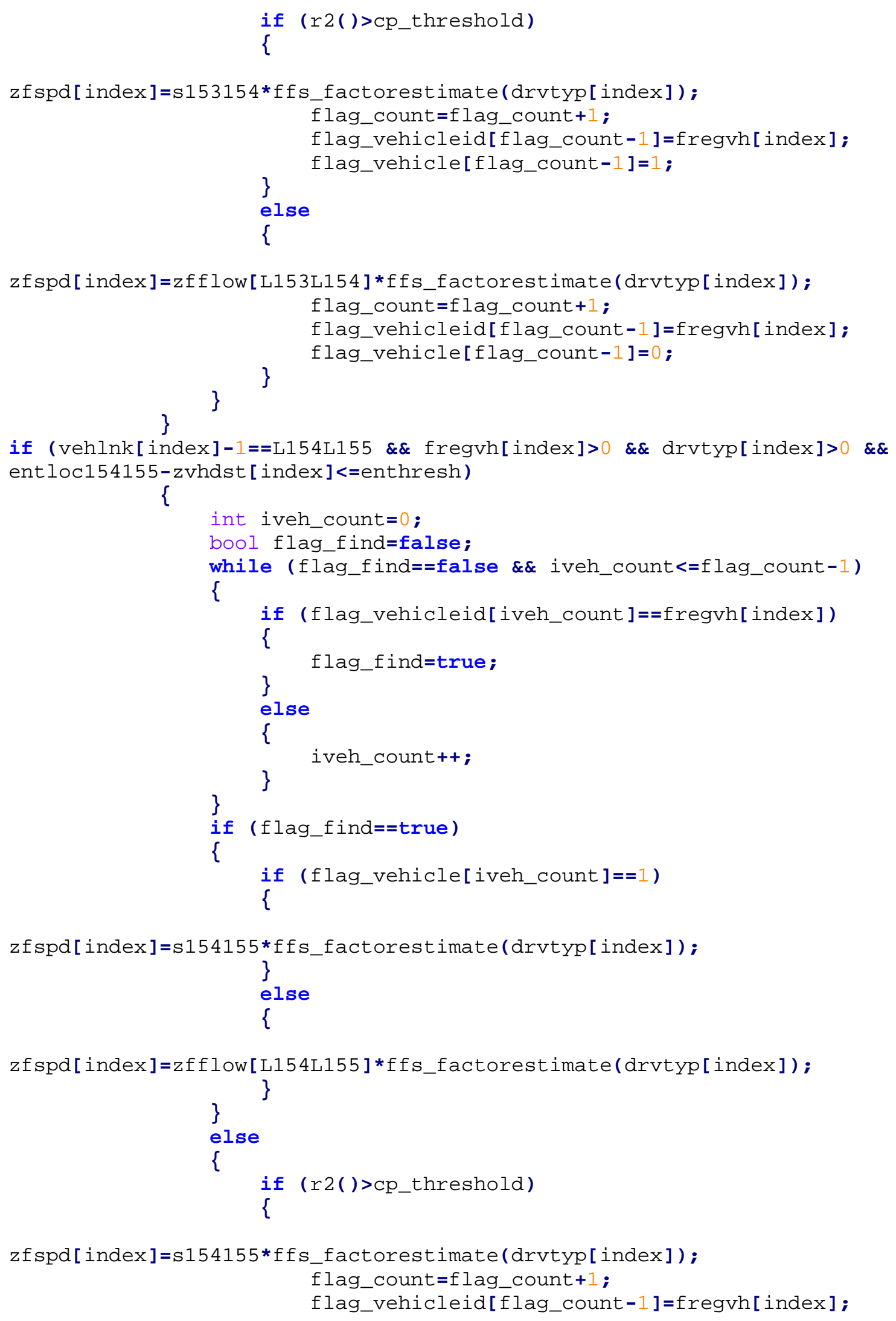




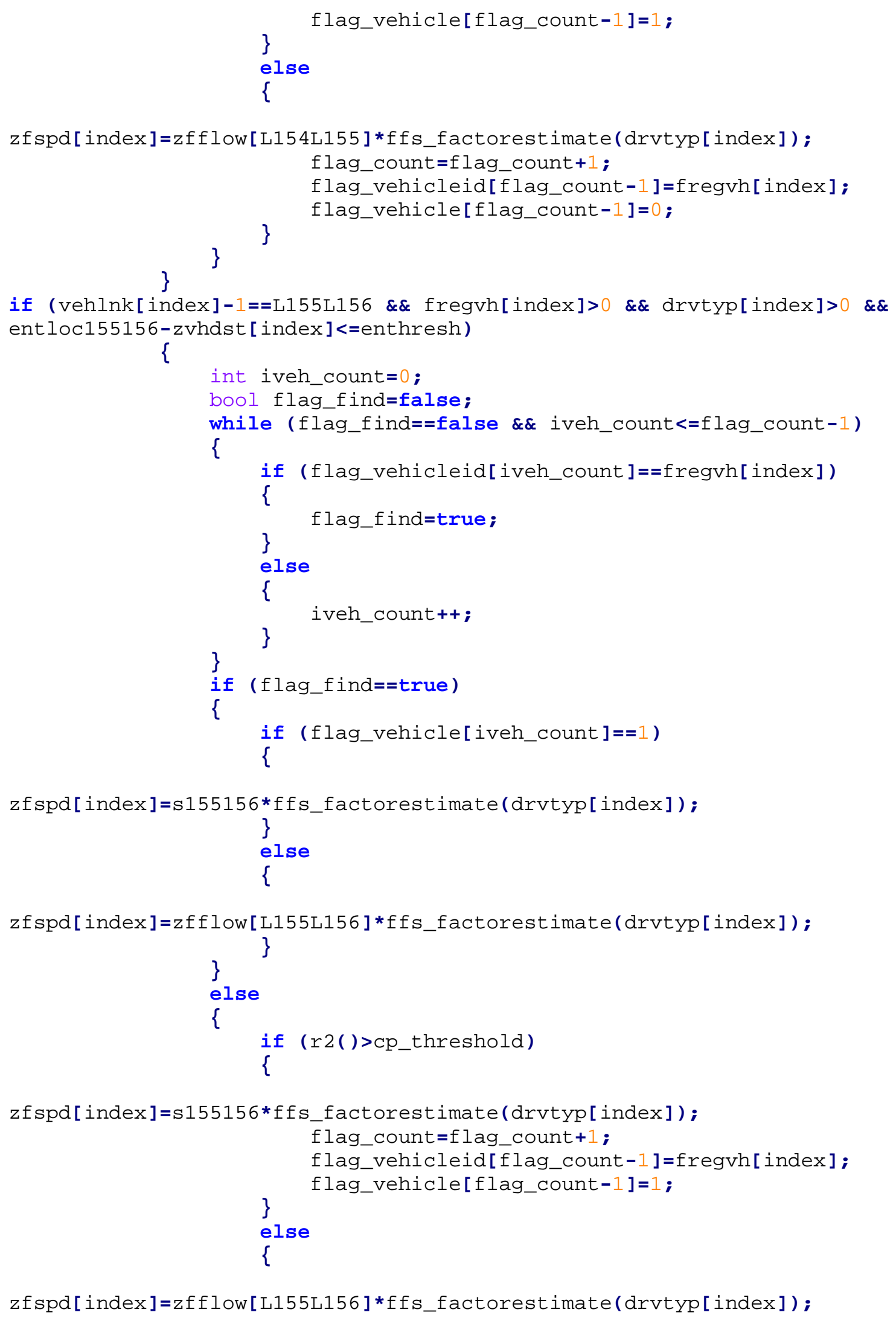


flag_count=flag_count +1 ;

flag_vehicleid[flag_count -1$]=f r e g v h[$ index] ;

flag_vehicle[flag_count -1$]=0$;

\}

\}

if (vehlnk[index]-1==L156L157 \&\& fregvh[index]>0 \&\& drvtyp[index]>0 \&\& entloc156157-zvhdst [index]<=enthresh) \{

int iveh_count $=\odot$;

bool flag_find=false;

while (flag_find==false \&\& iveh_count $<=f l a g \_c o u n t-1$ )

\{

if (flag_vehicleid[iveh_count]==fregvh[index])

\{

\}

flag_find=true;

else

\{

iveh_count++;

\}

\}

if ( $\left.f l a g \_f i n d==t r u e\right)$

\{

if (flag_vehicle[iveh_count]==1)

\{

zfspd[index]=s156157*ffs_factorestimate(drvtyp[index]);

\}

else

\{

zfspd[index]=zfflow[L156L157] *ffs_factorestimate(drvtyp[index]);

\}

\}

else

\{

if $\left(r 2()>c p \_t h r e s h o l d\right)$

\{

zfspd[index]=s156157*ffs_factorestimate(drvtyp[index]);

flag_count=flag_count +1 ;

flag_vehicleid[flag_count-1]=fregvh[index] ;

\}

flag_vehicle[flag_count -1$]=1$;

else

\{

zfspd[index]=zfflow[L156L157]*ffs_factorestimate(drvtyp[index]) ;

flag_count=flag_count +1 ;

flag_vehicleid[flag_count -1]=fregvh[index] ;

\}

flag_vehicle[flag_count -1]=॰ ; 
if (vehlnk[index]-1==L157L158 \&\& fregvh[index]>0 \&\& drvtyp[index]>0 \&\& entloc157158-zvhdst [index]<=enthresh)

$$
\{
$$

int iveh_count $=\odot$;

bool flag_find=false;

while (flag_find==false \&\& iveh_count $<=f l a g \_c o u n t-1$ )

\{

if (flag_vehicleid[iveh_count]==fregvh[index])

\{

\}

flag_find=true;

else

\{

\}

iveh_count++;

\}

if ( $f$ lag_find==true)

\{

if ( $f$ lag_vehicle[iveh_count]==1)

\{

zfspd[index]=s157158*ffs_factorestimate(drvtyp[index])；

\}

else

\{

zfspd[index]=zfflow[L157L158]*ffs_factorestimate(drvtyp[index]);

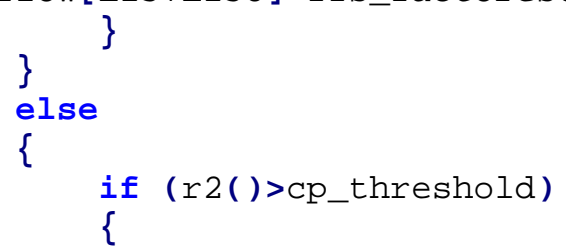

zfspd[index]=s157158*ffs_factorestimate(drvtyp[index]);

flag_count $=$ flag_count +1 ;

flag_vehicleid[flag_count -1]=fregvh[index] ;

\}

flag_vehicle[flag_count -1$]=1$;

\section{else}

\{

zfspd[index]=zfflow[L157L158] *ffs_factorestimate(drvtyp[index]);

flag_count $=$ flag_count +1 ;

flag_vehicleid[flag_count -1$]=f r e g v h[$ index] ;

flag_vehicle[flag_count -1$]=\odot$;

\}

\}

\}

if (vehlnk[index]-1==L158L159 \&\& fregvh[index]>0 \&\& drvtyp[index]>0 \&\& entloc158159-zvhdst [index]<=enthresh)

\{

int iveh_count $=\odot$;

bool flag_find=false;

while (flag_find==false \&\& iveh_count $<=f l a g \_c o u n t-1$ ) 


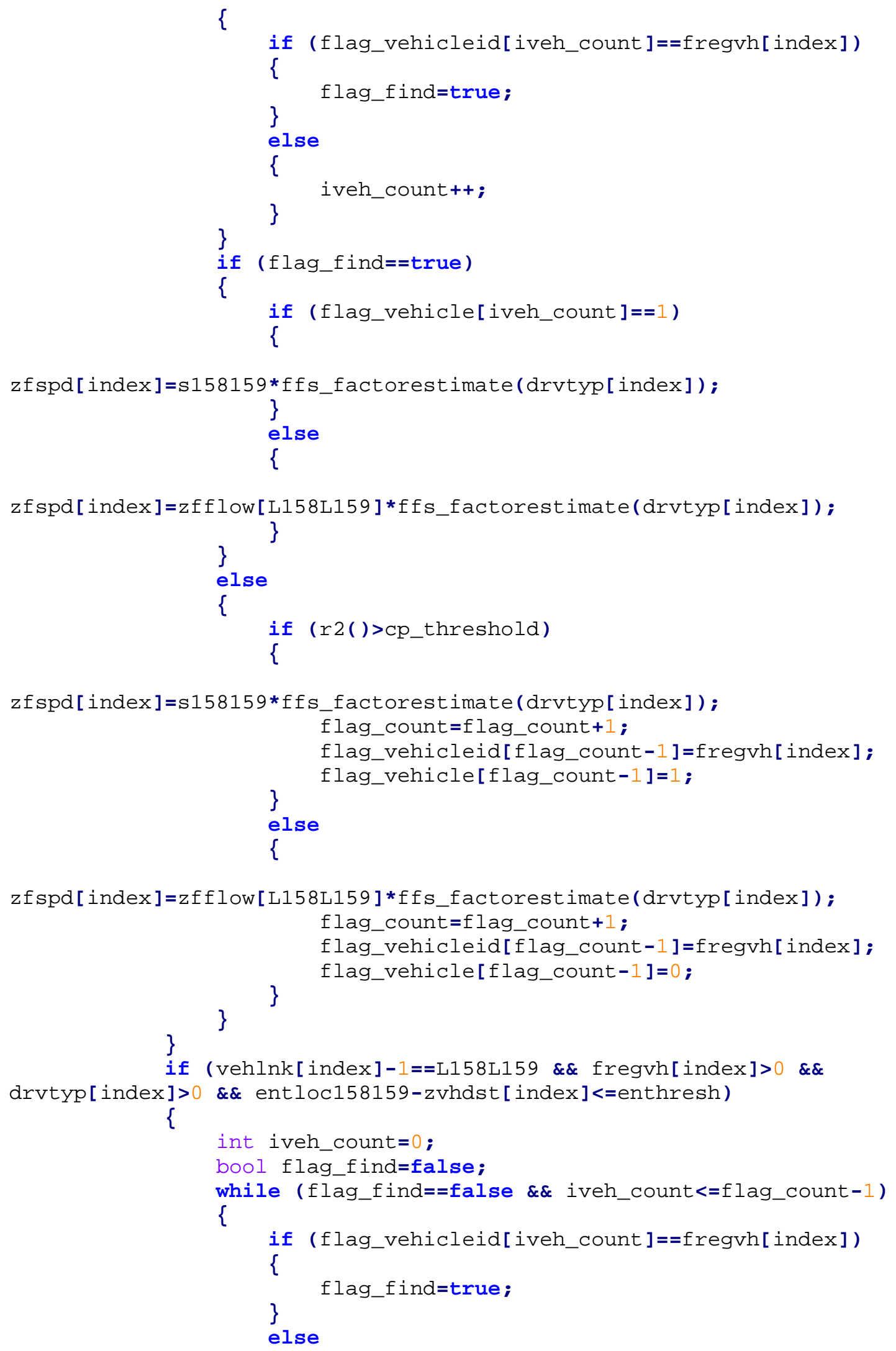




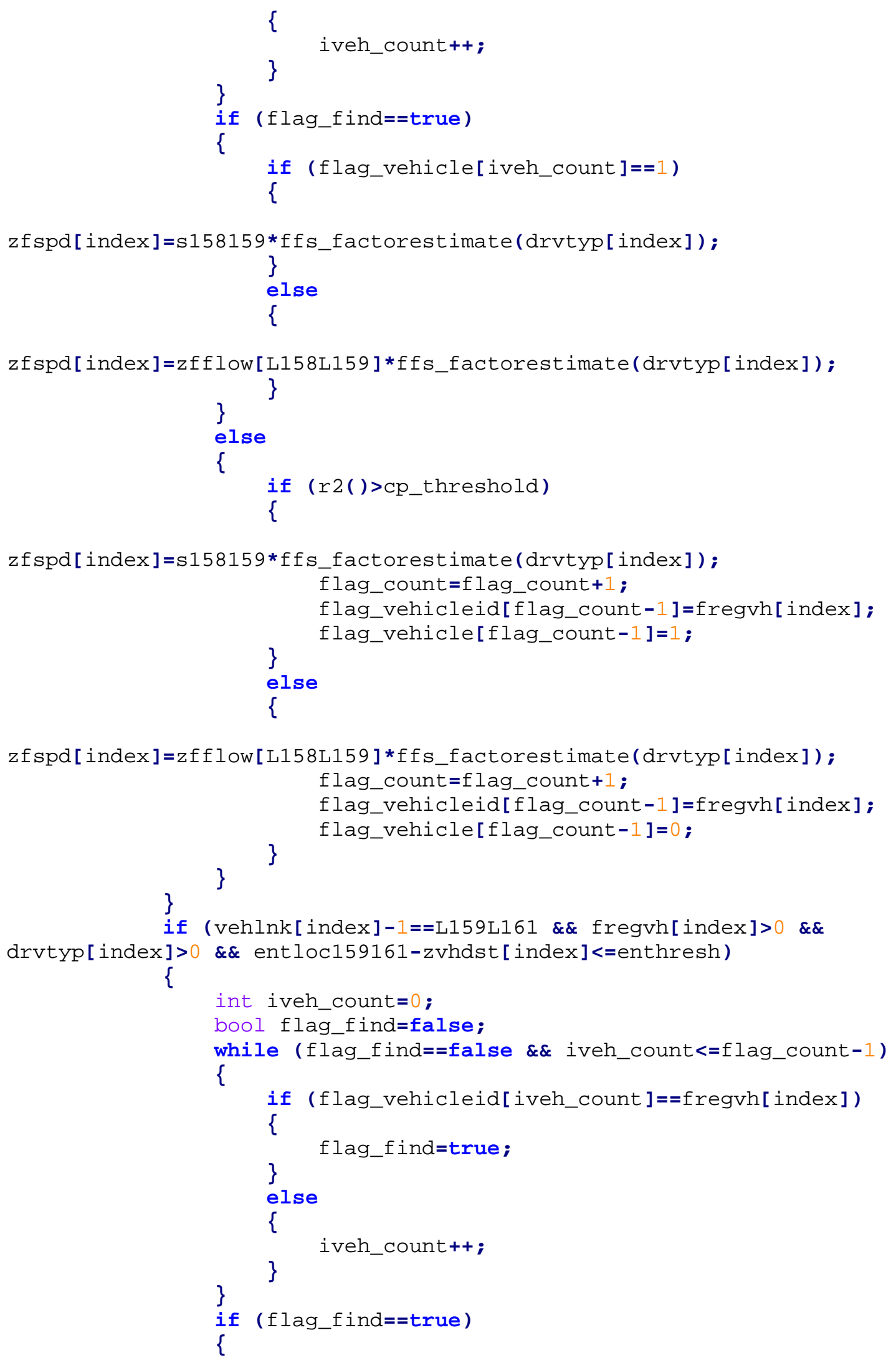




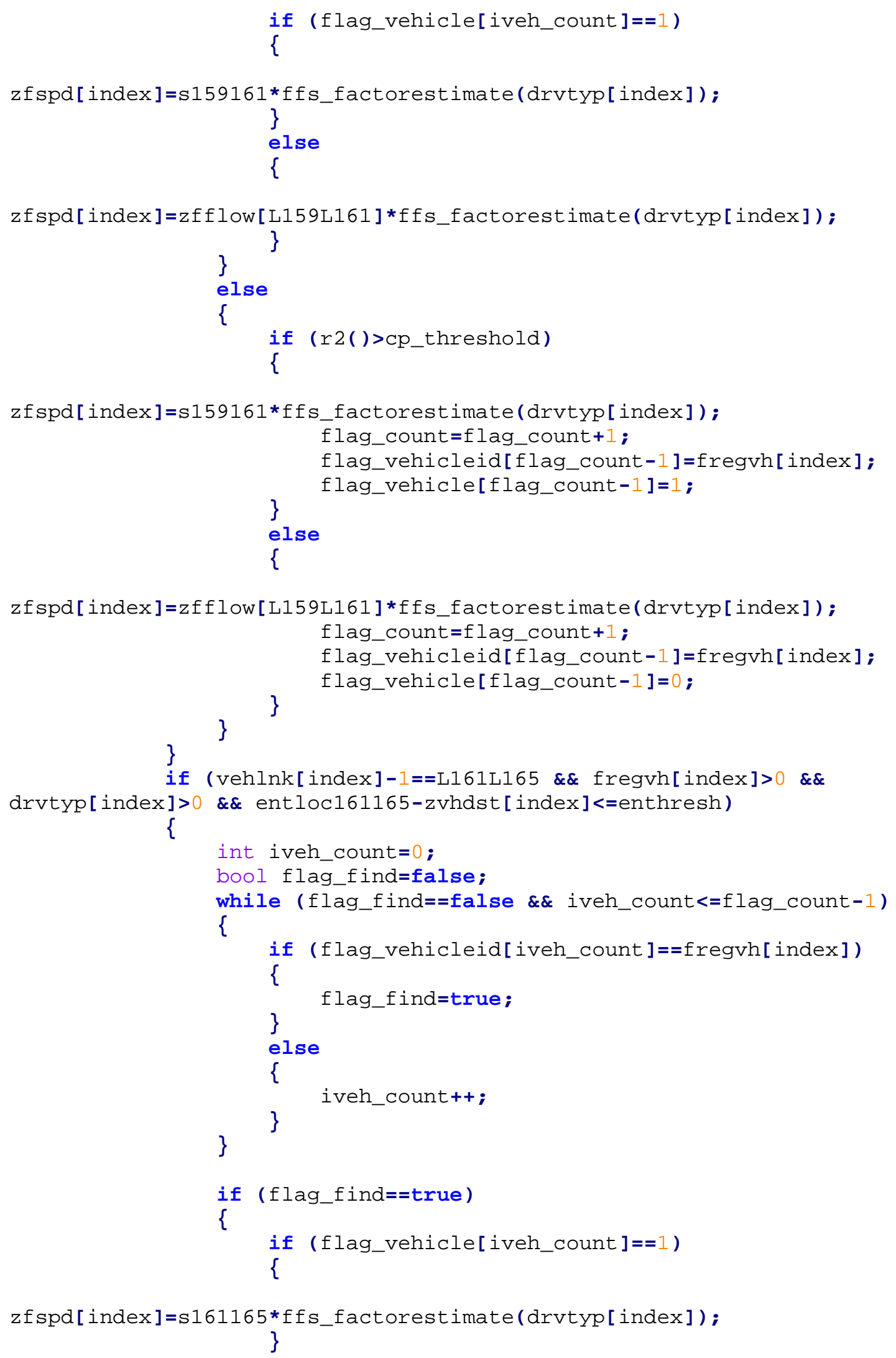




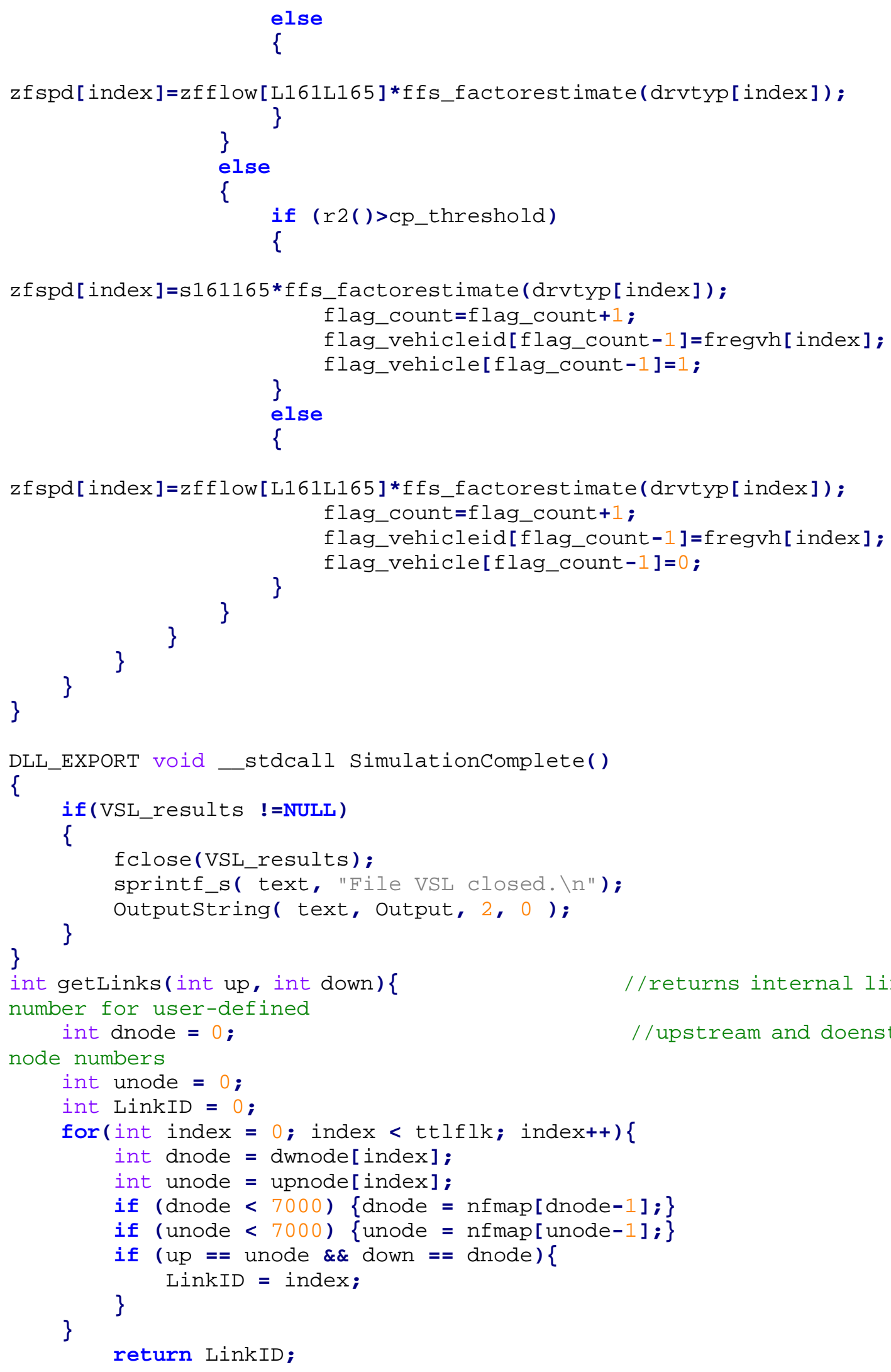


\}

int getDet(int link, int config)\{

$/ /$ returns the internal

detector id number for a detector

// on link and config

number of lanes

int detectorID $=0$;

if (fdetid[link] != $\odot$ ) \{

if $($ config $==1)\{$

\} detectorID $=$ fdetid[link $]-1$;

if $($ config $==2)\{$

\} detectorID $=($ fdetid $[$ link $]-1)+2$;

if $($ config $==3)\{$

\} detectorID $=($ fdetid $[$ link $]-1)+4$;

if $($ config $==4)\{$

\} detectorID $=($ fdetid $[$ link $]-1)+6$;

if (config $==5)\{$

\} detectorID $=($ fdetid $[$ link $]-1)+8$;

if (config $==6)\{$

\} detectorID $=($ fdetid $[$ link $]-1)+10$;

if $($ config $==7)\{$

\} detectorID $=($ fdetid $[$ link $]-1)+12$;

if $($ config $==8)\{$

\} detectorID $=($ fdetid $[$ link $]-1)+14$;

if $($ config $==9)\{$ \}

detectorID $=($ fdetid $[$ link $]-1)+16$;

\}

return detectorID;

\}

//finding the avergae of occupancy at different lanes at each detector location

void detectdata(int $\mathrm{d} 11$, int $\mathrm{d} 12$, int $\mathrm{d} 13$, int $\mathrm{d} 14$, int $\mathrm{d} 15$, int $\mathrm{d} 21$, int $\mathrm{d} 22$, int $d 23$, int $d 24$, int $d 31$, int $d 32$, int $d 33$, int $d 34$, int $d 41$, int $d 42$, int $d 43$, int $d 44$, int $d 51$, int $d 52$, int $d 53$, int $d 54$, int $d 55)\{$

float average $1=0$; average $1=(z f d o c c[d 11]+z$ fdocc $[$ d12 $]+z$ fdocc $[$ d13 $]+z f d o c c[d 14]+$ zfdocc $[\mathrm{d} 15]) / 5$;

for (int $i=2$; $i>0$; $i--)\{$ $\operatorname{detect} 1[i]=\operatorname{detect} 1[i-1]$;

\} $\operatorname{detect} 1[\odot]=$ average 1

$\operatorname{avg1}=(\operatorname{detect1}[\odot]+\operatorname{detect1}[1]+\operatorname{detect1}[2]) / 3 ;$

if (zclock ==60) avg1 = average1;

float average2 $=0$;

average $2=(z f d o c c[d 21]+z f d o c c[d 22]+z f d o c c[d 23]+z f d o c c[d 24]) / 4$;

for (int $i=2 ; i>0$; $i--)\{$

$\operatorname{detect} 2[i]=\operatorname{detect} 2[i-1]$; 


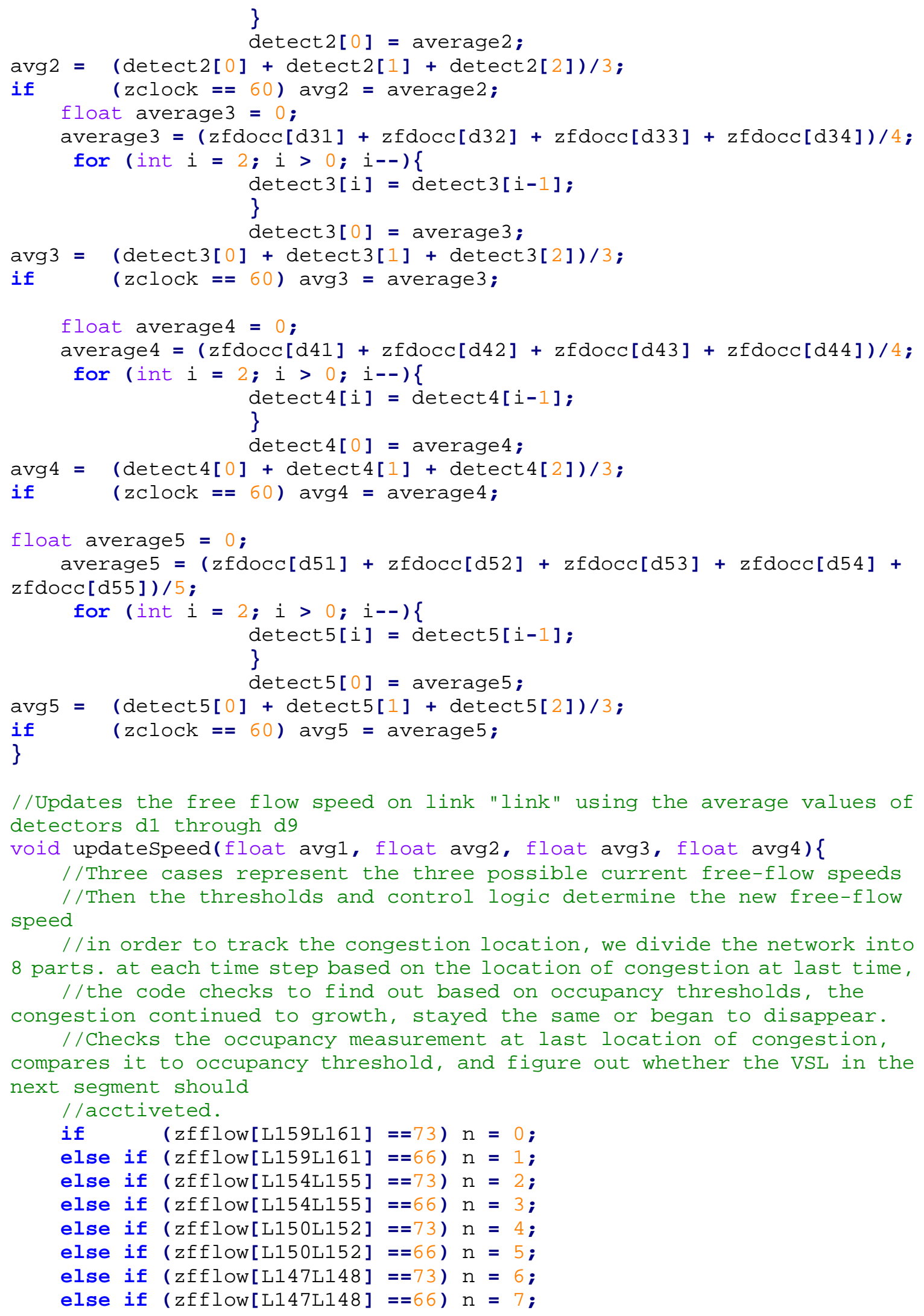




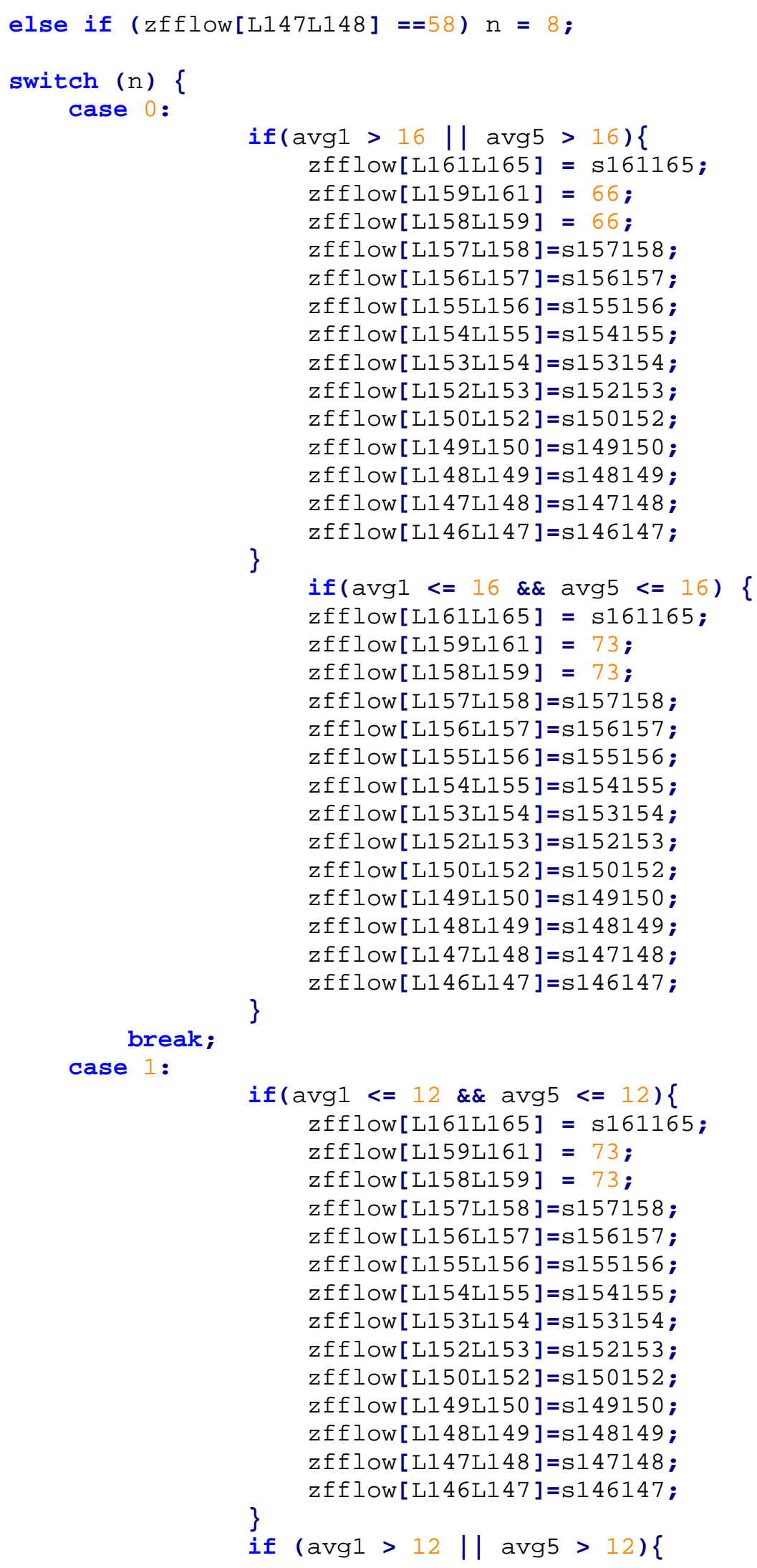




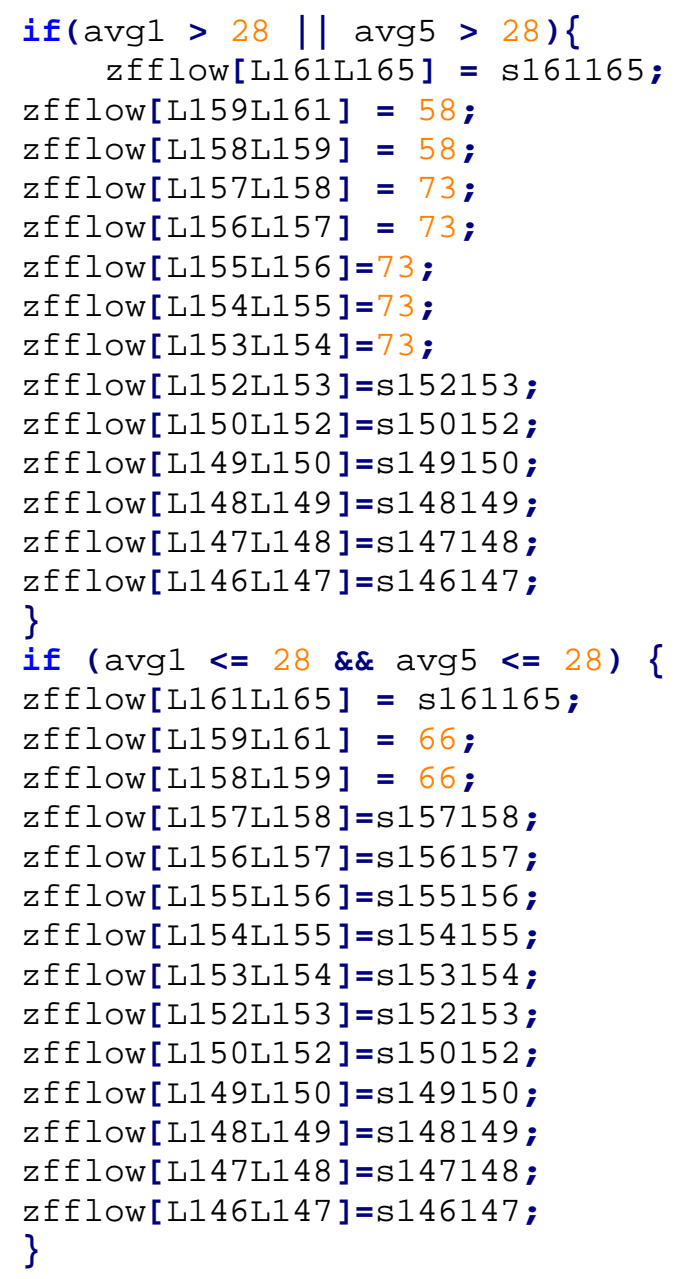




$$
\begin{aligned}
& \text { zfflow[L157L158] = 66; } \\
& \text { zfflow }[\text { L156L157] = 66: } \\
& \text { zfflow [L155L156]=66; } \\
& \text { zfflow [L154L155]=66; } \\
& \text { zfflow [L153L154]=66; } \\
& \text { zfflow [L152L153]=s152153; } \\
& \text { zfflow [L150L152]=s150152; } \\
& \text { zfflow [L149L150]=s149150; } \\
& \text { zfflow [L148L149] =s148149; } \\
& \text { zfflow [L147L148]=s147148; } \\
& \} \\
& \text { zfflow [L146L147]=s146147; } \\
& \text { if(avg5 >= } 25 \text { \&\& avg2 <= 16) \{ } \\
& \text { zfflow[L161L165] = s161165; } \\
& \text { zfflow [L159L161] = 58; } \\
& \text { Zfflow [L158L159] = 58; } \\
& \text { zfflow [L157L158] = 73; } \\
& \text { Zfflow [L156L157] = 73; } \\
& \text { Zfflow [L155L156]=73; } \\
& \text { zfflow [L154L155]=73; } \\
& \text { zfflow [L153L154]=73; } \\
& \text { zfflow [L152L153]=s152153; } \\
& \text { zfflow [L150L152] =s150152; } \\
& \text { zfflow [L149L150]=s149150; } \\
& \text { zfflow [L148L149]=s148149; } \\
& \text { zfflow [L147L148]=s147148; } \\
& \text { \} } \\
& \text { zfflow [L146L147]=s146147; }
\end{aligned}
$$

break:

case 3 :

$$
\begin{aligned}
& \text { if }(\operatorname{avg} 2<=12)\{ \\
& \text { zfflow[L161L165] = s161165; } \\
& \text { zfflow [L159L161] = 58; } \\
& \text { zfflow [L158L159] = 58; } \\
& \text { zfflow [L157L158] = 73; } \\
& \text { Zfflow }[\text { L156L157] = 73; } \\
& \text { zfflow [L155L156] =73; } \\
& \text { zfflow [L154L155]=73; } \\
& \text { zfflow [L153L154]=73; } \\
& \text { zfflow [L152L153]=s152153; } \\
& \text { zfflow [L150L152] =s150152; } \\
& \text { zfflow [L149L150] =s149150; } \\
& \text { zfflow [L148L149]=s148149; } \\
& \text { zfflow [L147L148]=s147148; } \\
& \text { zfflow [L146L147]=s146147; } \\
& \text { \} } \\
& \text { if }(\operatorname{avg} 2>12)\{ \\
& \text { if }(\operatorname{avg} 2>28)\{ \\
& \text { zfflow[L161L165] = s161165; } \\
& \text { zfflow [L159L161] = s159161; } \\
& \text { zfflow[L158L159] = s158159; } \\
& \text { Zfflow [L157L158] = s157158; } \\
& \text { zfflow[L156L157] = s156157; } \\
& \text { zfflow [L155L156] }=58 \text {; } \\
& \text { zfflow [L154L155]=58; }
\end{aligned}
$$




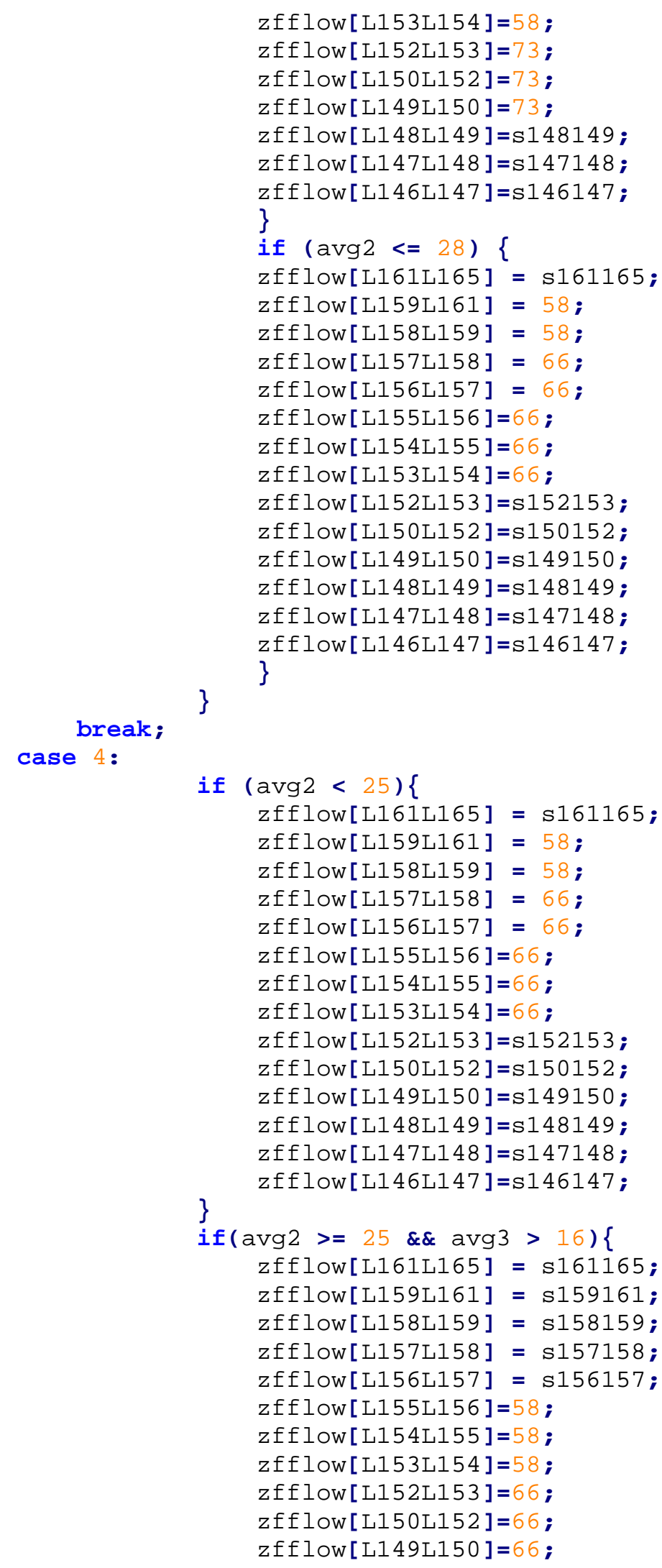




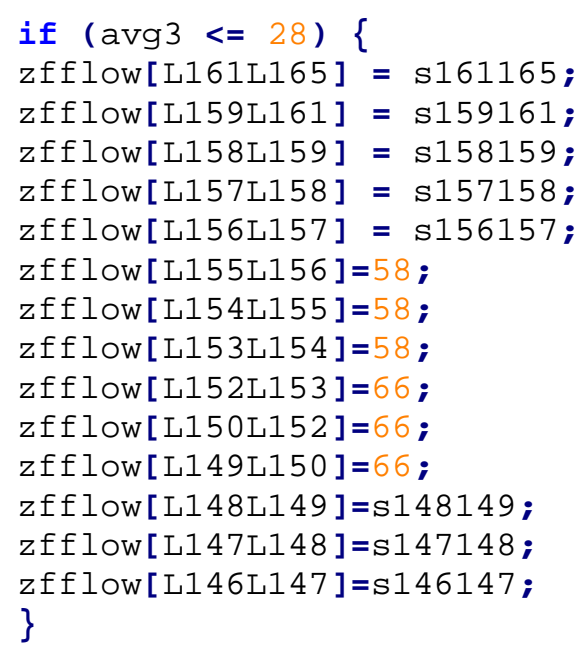




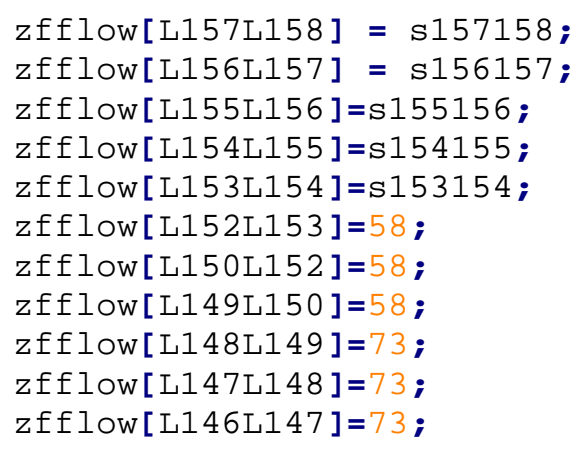

break;

case 7:

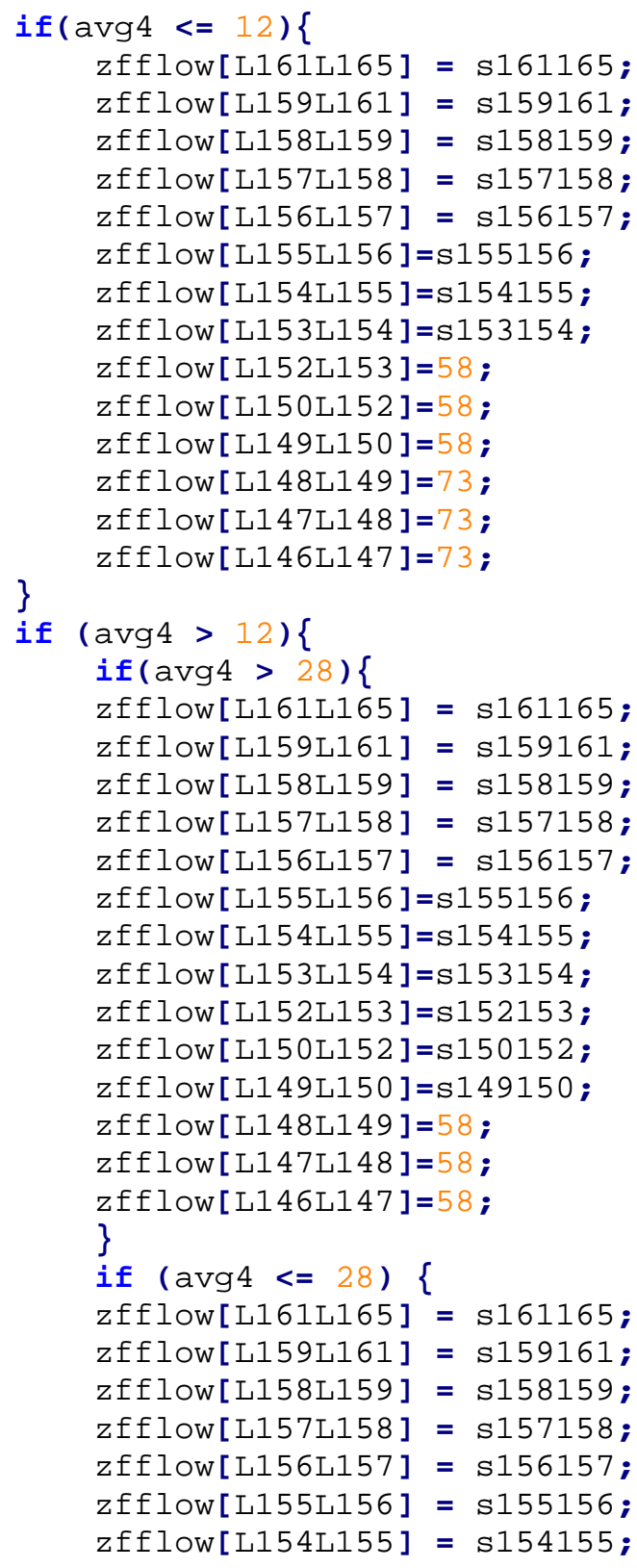




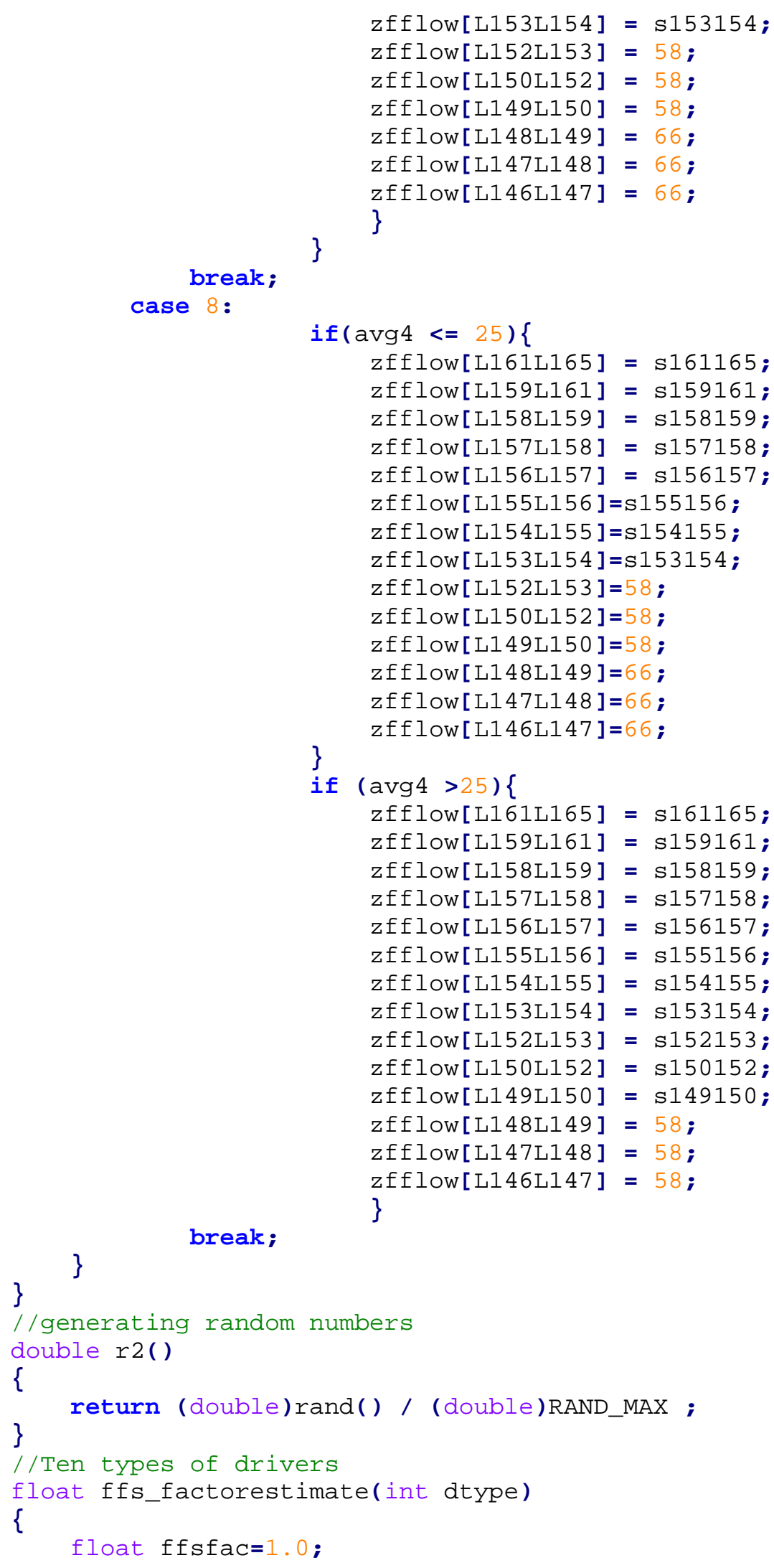




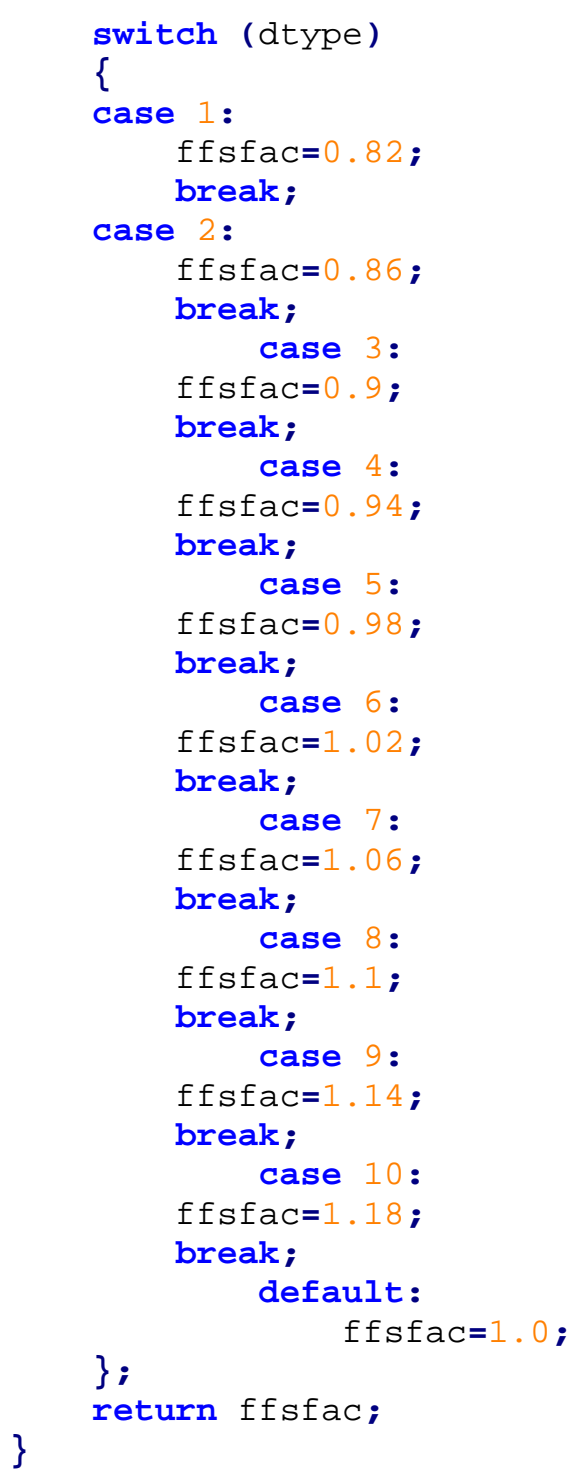


VITA

\section{ALI DARROUDI}

EDUCATION, EMPLOYMENT, AND AFFILIATIONS

2003 - $2007 \quad$ B.S., Civil Engineering

Amirkabir University of Technology (Tehran Polytechnic)

Tehran, Iran

2007 - $2010 \quad$ M.S., Civil Engineering

Sharif University of Technology

Tehran, Iran

2007 - $2010 \quad$ Transportation Engineer Thinking New Approaches (TNA) Consulting Co.

Tehran, Iran

2010 - $2014 \quad$ PhD in Civil Engineering

Florida International University

Miami, Florida

2010-2014 Graduate Research Assistant

Lehman Center for Transportation Research, FIU

Miami, Florida

2010 - Present Student member

Institute of Transportation Engineers

2011 - Present Student member

Chi Epsilon Honor Society

\section{PUBLICATIONS AND PRESENTATIONS}

- Hadi, M., Elefteriadou, L., Darroudi, A., Letter, C., Xiao, Y., and Kondyli, A. (2014). Investigation of ADTM Strategies to Reduce the Probability of Breakdown. Final Report Prepared for STRIDE by the Florida International University and University of Florida.

- Darroudi, A., Hadi, M., Xiao, Y., Letter, C., Kondyli, A., and Elefteriadou, L. (2014). Adaptive Variable Speed Limit Strategy to Reduce Shockwave Upstream of Bottlenecks. Presented (Poster) at the $2^{\text {nd }}$ Annual UTC Conference for the Southeastern Region, Atlanta, Georgia. 
- Hadi, M., Elefteriadou, L., Kondyli, A., Xia, Y., Letter, C., and Darroudi, A. (2013). "Investigation of ATDM Strategies to Reduce the Probability of Breakdown”. Presented at the $1^{\text {st }}$ Annual UTC Conference for the Southeastern Region, Orlando, Florida.

- Darroudi, A. (2012). Solving Nonlinear Multicommodity Network Flow Problem by Dynamic Penalty Function Method. Presented at the $1^{\text {st }}$ International Industrial Engineering Conference, Istanbul, Turkey.

- Darroudi, A., and Aashtiani, H.Z. (2012). Solving Nonlinear Multicommodity Network Flow Problem by Penalty Function Methods. Presented (Poster) at the INFORMS Conference, Phoenix, Arizona. 